\author{
Universidade de São Paulo \\ Instituto de Física de São Carlos
}

Paulo Eduardo de Faria Junior

\title{
Development and application of the k.p method to investigate spin and optical properties of semiconductor nanostructures
}



Paulo Eduardo de Faria Junior

\section{Development and application of the k.p method to investigate spin and optical properties of semiconductor nanostructures}

Thesis submitted to the Physics Graduate Program at the São Carlos Institute of Physics, University of São Paulo, to obtain the degree of Doctor of Sciences.

Concentration area: Applied Physics

Option: Computational Physics

Advisor: Prof. Dr. Guilherme Matos Sipahi

Co-advisor: Prof. Dr. Igor Žutić

Reviewed version

(Original version available on the Program Unit)

São Carlos

2016 
AUTHORIZE THE REPRODUCTION AND DISSEMINATION OF TOTAL OR PARTIAL COPIES OF THIS THESIS, BY CONVENCIONAL OR ELECTRONIC MEDIA FOR STUDY OR RESEARCH PURPOSE, SINCE IT IS REFERENCED.

Cataloguing data reviewed by the Library and Information Service of the IFSC, with information provided by the author

Faria Junior, P. E.

Development and application of the k.p method to investigate spin and optical properties of

semiconductor nanostructures / P. E. Faria Junior;

advisor G. M. Sipahi; co-advisor I. Zutic - reviewed version -- São Carlos 2016.

$140 \mathrm{p}$.

Thesis (Doctorate - Graduate Program in

Computacional Physics) -- Instituto de Física de São Carlos, Universidade de São Paulo - Brasil, 2016.

1. Spin lasers. 2. Polytypism. 3. Wurtzite. 4. Semiconductors. 5. k.p method. I. Sipahi, G. M., advisor. II. Zutic, I., co-advisor. III. Title. 
To my parents, Paulo and Márcia. And to all of those who believe that knowledge can change the world. 



\section{Acknowledgments}

I dedicate this thesis to my parents Paulo and Márcia. I could not put into words all the love and gratitude that I have for them.

Among the people that helped me accomplish this thesis, I would like to thank:

- Some special people close to me during this process: Débora, Danilo, Nivaldo and Ana Paula for their great support and special thanks to Jessica for her love and patience.

- The friends at IFSC that I had the opportunity to interact socially and scientifically throughout the years. Special mention to Tiago, Denis and Carlos, for sharing similar research interests and helping with discussions about physics.

- Friends that I made during my stay in Buffalo, especially, Ballav, Jeongsu, Jim Parry and Jimmy P.

- Prof. Dr. Guilherme Matos Sipahi for his supervision, numerous discussions and the great scientific opportunities he provided during this $\mathrm{PhD}$.

- Prof. Dr. Igor Žutić for all his effort regarding my scholarship projects, for introducing me to the field of spintronics, all the nice discussions and for kindly hosting me in Buffalo multiple times. I also acknowledge Prof. Žutić's group members for the great social and scientific interactions.

- Dr. Nils Gerhardt for the great discussions about semiconductor (spin) lasers and for kindly hosting me in Bochum. I also want to acknowledge Nils' group in Bochum, especially Prof. Martin Hoffman and Markus Lindemann, for the nice discussions and the lab tour.

- Prof. Dr. Jaroslav Fabian for all the fruitful discussions and insights, for bringing me closer to the $a b$ initio world and for kindly hosting me in Regensburg. I also offer my gratitude to the people in Prof. Fabian's group, especially to Dr. Martin Gmitra for the patience to clarify many questions I had about $a b$ initio calculations.

- Prof. Dr. José Carlos Egues for kindly taking the responsibility of my scholarship and for the nice discussions and suggestions during my $\mathrm{PhD}$ qualifying exam.

- Prof. Dr. Fernando Iikawa and Prof. Dr. Iouri Poussep for being part of my PhD qualification committee and providing helpful feedback to this thesis.

- Prof. Dr. Erasmo de Andrada e Silva, Prof. Dr. Iouri Poussep, Prof. Dr. Victor LopezRichard and Prof. Dr. José Carlos Egues for being part of my PhD evaluation committee and providing helpful feedback to improve this thesis.

- The people at IFSC for their tremendous help and dedication. Special thanks, in alphabetic order, to Ailton, Cris, Italo, Maria Cristina, Ricardo, Silvio and Sonia. 
- FAPESP (São Paulo Research Foundation) for the financial support of the PhD scholarship (grant No. 2012/05618-0) and the BEPE scholarship (grant No. 2013/23393-8). 
"The first principle is that you must not fool yourself, and you are the easiest person to fool." -Richard P. Feynman (1918 - 1988)

"I think it is much more interesting to live not knowing than to have answers that might be wrong." - Richard P. Feynman (1918 - 1988)

"Individual curiosity, often working without practical ends in mind, has always been a driving force for innovation." -Frederick SeItZ (1911 - 2008) 



\section{Abstract}

FARIA JUNIOR, P. E. Development and application of the k.p method to investigate spin and optical properties of semiconductor nanostructures. 2016. 140 p. Thesis (Doctor in Science) - Instituto de Física de São Carlos, Universidade de São Paulo, São Carlos, 2016.

Many observable properties of semiconductor systems, such as transport and optical transitions, are manifestations of their underlying electronic band structures, i. e., the energy levels that electrons may have in the semiconductor. Among the theoretical approaches to calculate the band structure, the k.p method is a versatile framework that can be extended to deal with confined systems, overcoming the computational limitations of first principles methods. In this thesis, we develop and apply k.p Hamiltonians to investigate spin and optical physical phenomena in unconventional semiconductor systems. Specifically, we addressed three different topics: spin lasers, polytypism in III-V semiconductors and spin-orbit coupling effects in wurtzite materials. For spin lasers, we investigate the behavior of their active region, in a VCSEL geometry, based on GaAs/AlGaAs zinc-blende quantum wells by calculating the spin-dependent gain coefficient. Assuming spin polarized electrons, our calculations showed the spin-filtering and the threshold reduction features found in experiments and by the conventional rate equation approach in the steady-state operation. Motivated by experimental evidence of enhanced dynamic operation for light polarization because of anisotropies in the semiconductor system, we calculate the birefringence coefficient of the active region under uniaxial strain. Our calculations showed that, even for a small value of applied strain, the birefringence coefficient can easily exceed $200 \mathrm{GHz}$. In fact, our predictions were experimentally demonstrated for values up to 250 $\mathrm{GHz}$ in similar GaAs/AlGaAs spin VCSELs. For the polytypism topic, we develop a k.p model combined with the envelope function approximation to investigate the polytypism in III-V semiconductor systems with mixed zinc-blende and wurtzite crystal structures. We apply our model for InP polytypic quantum wells to investigate quantum confinement and strain effects. We then extended this polytypic model to include the explicit coupling between the conduction and the valence bands in order to investigate optical properties in InP polytypic superlattices. For pure phase nanowires, modeled with bulk calculations and the optical confinement, we can see the same experimental trends regarding the light polarization, i. e., zinc-blende phase favors light polarization along the nanowire axis while wurtzite phase favors the polarization perpendicular to the axis. Including the crystal phase mixing and the quantum confinement effects, we obtain the degree of light polarization ranging from pure zinc-blende to pure wurtzite nanowires and, more specifically, that this degree of polarization is very sensitive to the size of zinc-blende regions, a feature that is also observed in photoluminescence measurements. Finally, we develop a realistic k.p Hamiltonian, with parameters obtained from ab initio band structures, to investigate electronic properties and spin-orbit coupling effects in InAs and InP semiconductors with wurtzite structure. Our $8 \times 8$ k.p model describes the conduction and the valence bands, including spin, around the energy gap. We also include the $k$-dependent spin-orbit coupling term, usually neglected in the literature, to correctly describe the bulk inversion asymmetry of wurtzite structure. We show that all the investigated energy bands have a spin expectation value 
that follows a Rashba-like spin texture, with either clockwise or counter clockwise orientation. We emphasize that all the $a b$ initio features of band structure, spin splittings and spin orientation were systematically checked to provide the best parameter sets. Using the $8 \times 8$ k.p Hamiltonian, we calculated the density of states and predicted the carrier density as a function of the Fermi energy. We also provide an analytical approach for conduction band and a compact description for the valence bands, however, the $8 \times 8$ Hamiltonian is the best approach to recover the $a b$ initio calculations around a large region of the first Brillouin zone.

Keywords: Spin lasers. Polytypism. Wurtzite. Semiconductors. k.p method. 


\section{Resumo}

FARIA JUNIOR, P. E. Desenvolvimento e aplicação do método k.p para investigar propriedades óticas e de spin em nanostruturas semicondutoras. 2016. 140 p. Tese (Doutorado em Ciências) - Instituto de Física de São Carlos, Universidade de São Paulo, São Carlos, 2016.

Diversas propriedades observáveis de sistemas semicondutores, como transporte e transições óticas, são manifestações de suas estruturas de bandas eletrônica, isto é, os níveis energéticos que elétrons podem ocupar no semicondutor. Entre as abordagens teóricas para o cálculo de estrutura de bandas, o método k.p é uma ferramenta versátil que pode ser estendida para tratar sistemas confinados, superando as limitações computacionais de métodos de primeiros princípios. Nesta tese, nós desenvolvemos e aplicamos Hamiltonianos k.p para fenômenos físicos óticos e de spin em sistemas semicondutores não convencionais. Especificamente, nós consideramos três tópicos diferentes: lasers de spin, politipismo em semicondutores III-V e efeitos do acoplamento spin-órbita em materiais com estrutura cristalina wurtzita. Para os lasers de spin, investigamos o comportamento de sua região ativa, em uma geometria VCSEL, baseada em poços quânticos de zinc-blende GaAs/AlGaAs através do cálculo do coeficiente de ganho dependente de spin. Assumindo elétrons com polarização de spin, nossos cálculos mostraram as características de filtro de spin e de redução do limiar de laser encontradas em experimentos e pela abordagem convencional de equações de taxa no regime estacionário. Motivados pelas evidências experimentais de operação dinâmica mais veloz para a polarização da luz devido às anisotropias do sistema, calculamos o coeficiente de birrefringência para a região ativa sob efeito de uma tensão uniaxial. Nossos cálculos mostraram que, mesmo para um pequeno valor de tensão aplicada, o coeficiente de birrefringência pode facilmente exceder $200 \mathrm{GHz}$. Na realidade, nossas predições foram demonstradas experimentalmente para valores de até $250 \mathrm{GHz}$ em um dispositivo VCSEL de spin de GaAs/AlGaAs similar ao nosso sistema estudado. Para o politipismo, desenvolvemos um modelo k.p combinado com a aproximação da função envelope para investigar o politipismo em sistemas semicondutores III-V com mistura de estruturas cristalinas zinc-blende e wurtzita. Aplicamos o modelo para poços quânticos politípicos de InP para investigar efeitos de confinamento quântico e de tensão. Também estendemos esse modelo politípico para incluir explicitamente o acoplamento entre as bandas de condução e valência com o intuito de investigar propriedades óticas em superredes politípicas de InP. Para nanofios com fase cristalina pura, modelados por cálculos na forma bulk com inclusão do confinamento ótico, observamos as mesmas características experimentais para a polarização da luz, isto é, a fase zinc-blende favorece a polarização da luz ao longo do eixo do nanofio enquanto a fase wurtzita favorece a polarização perpendicular ao eixo. Incluindo a mistura cristalina e os efeitos de confinamento quântico, obtemos o grau de polarização linear variando entre os valores de nanofios puros de zinc-blende e wurtzita e, mais especificamente, que esse grau de polarização é muito sensível ao tamanho das regiões de zinc-blende, uma característica também observada em medidas de fotoluminescência. Finalmente, desenvolvemos um Hamiltoniano k.p realista, com parâmetros obtidos de estruturas de bandas por primeiros princípios, para investigar propriedades eletrônicas e efeitos do acoplamento spin-órbita em materiais semicondutores de InAs 
e InP com estrutura cristalina wurtzita. Nosso modelo k.p $8 \times 8$ descreve as bandas de condução e valência, incluindo spin, em torno da energia de gap. Nós também incluímos o termo de acoplamento spin-órbita dependente de $k$, geralmente desprezado na literatura, para descrever corretamente a assimetria de inversão de bulk da estrutura wurtzita. Mostramos que todas as bandas de energia investigadas possuem um valor esperado de spin que segue a textura de spin do tipo Rashba, com orientação no sentido horário ou anti-horário. Nós enfatizamos que todas as características da estrutura de bandas, abertura de spin e orientação de spin dos cálculos de primeiros princípios foram sistematicamente checadas para fornecer o melhor conjunto de parâmetros. Usando o Hamiltoniano k.p $8 \times 8$, calculamos a densidade de estados e obtemos a densidade de portadores como função da energia de Fermi. Fornecemos também uma abordagem analítica para a banda de condução e uma descrição compacta para a banda de valência, no entanto, o Hamiltoniano $8 \times 8$ é a melhor abordagem para modelar os cálculos de primeiros princípios em uma ampla região da primeiro zona de Brillouin.

Palavras-chave: Lasers de spin. Politipismo. Wurtzita. Semicondutores. Método k.p. 


\section{List of Figures}

Figure 1.1 - Band structure for the (a) free electron model, (b) a metal and (c) a semiconductor or insulator. The energy bands in Figs. (b) and (c) are indicated in different colors and labeled, for simplicity, as bands 1 and 2. The filled circles indicate occupied states, therefore metals show a partially occupied band with empty states for electrons to access, while semiconductors and insulators have a fully occupied band. The next available band is separated by the energy gap, a forbidden region of energy. . . . . . . . . . . . . . . . .

Figure 1.2 - The bucket model analogy for (a) conventional and (b) spin lasers. Water flowing into the buckets represent the carrier injection and water flowing out of the bucket indicate light emission. Lasing threshold is achieved when the water level reaches the large opening of the bucket. The leaks indicate the light emitted in the no lasing regime. Small holes between the two sides of the bucket in Fig. (b) represent the mixing of spin populations due to spin relaxation. Graphical description of input vs output (injection vs emission) for (c) conventional and (d) spin lasers. The lasing threshold is indicated by the point $T$, for conventional lasers, and by the points $T_{a}$ and $T_{b}$ for spin lasers. The dark area shows the spin-filtering regime between $T_{a}$ and $T_{b} \ldots \ldots \ldots \ldots$

Figure 1.3 - (a) Possible design of a VCSEL, including the top (tDBR) and bottom (bDBR) DBRs, the top (tC) and bottom (bC) contacts and the resonant cavity (Cav). The arrows inside the cavity indicate the light feedback of trapped photons. The output light is emitted upwards and the twisted arrows indicate positive and negative helicities for light polarization. Since the bDBR is not a perfect mirror, light can also be emitted downwards. In the inset, the cavity and the active region (AR) are shown in detail. The lengths of the whole VCSEL, the cavity and the active region are indicated by $\mathrm{L}_{\mathrm{V}}, \mathrm{L}_{\mathrm{C}}$ and $\mathrm{L}_{\mathrm{W}}$, respectively, We also show a possible scheme for applied mechanical strain at the substrate. (b) The effect of mechanical strain along the VCSEL. The strain field propagates upwards and loses its amplitude. In the scale of the active region, compared to the whole VCSEL, it is reasonable to imagine a small and constant strain.

Figure 1.4 - (a) ZB conventional unit cell. (b) ZB primitive unit cell. (c) ZB FBZ. (d) WZ conventional unit cell. (e) WZ primitive unit cell. (f) WZ FBZ. The atoms are numbered to simplify the connection between conventional and primitive unit cells. The arabic numbering indicate atoms of type 1 while roman numbering indicate atoms of type 2 . In the FBZs, the shaded region is the irreducible wedge created by the high-symmetry points. . . . . . . . . . . . . . . . . 
Figure 2.1 - Self-consistent fitting scheme to obtain the k.p parameters based on $a b$ initio band structures, used in Ref.(127). The fitting approach provides a number of different parameter sets that are compared by residue analysis. The parameter set with a smaller residue is used as an input for the numerical fitting until the residue converges. . . . . . . . . . . . .

Figure 2.2 - One-dimension external potential (a) in a single material A and (b) for quantum well profile created by the regions $\mathrm{B} / \mathrm{A} / \mathrm{B}$. Regions $\mathrm{A}$ and $\mathrm{B}$ can be two different materials or the same material with different crystal structures. The whole system has a dimension $L \ldots \ldots$. . . . . . . .

Figure 2.3 - A qualitatively description of the QC effect in energy bands. (a) Bulk energy bands. (b) Zone folding. (c) Energy shift and mini gaps induced in the energy bands. (d) Minibands in strong QC. (e) Minibands and subbands.

Figure 3.1 - Quasi-equilibrium configuration in the band structure due to carrier injection (a) without spin polarization and (b) assuming spin-polarized carriers. The red arrows indicate spin up electrons and the blue arrows indicate spin down electrons. . . . . . . . . . . . . . . . .

Figure 3.2 - (a) Band structure, (b) DOS and (c) Fermi-Dirac occupancy without spin polarization for the two-band model. We used $m_{c}^{*}=0.04, m_{v}^{*}=0.4$ and $E_{g}=1.0 \mathrm{eV}$. For the occupancy we assumed $T=300 \mathrm{~K}$. Vertical arrows indicate (a) the energy gap and (b) the differences of chemical potential energies, $\Delta \mu=\mu_{C}-\mu_{V} \ldots \ldots \ldots \ldots \ldots$

Figure 3.3 - (a) Gain coefficient at $T=0 \mathrm{~K}$, i. e., the negative value of the absorption, without injected carriers and (b) gain coefficient at $T=300 \mathrm{~K}$ with and without spin polarized electrons for different carrier densities. Different values of $\gamma$ are used in (a). In (b), we fixed $\gamma=0.01$ and considered different values of carrier densities. For the constant $C_{0}$ [equation (3.35)], we used $P=10 \mathrm{eV} \AA, L=80 \AA$ and $n_{r}=3 . \ldots \ldots$. . . . . . 


\section{List of Abbreviations and Acronyms}

\begin{tabular}{|c|c|}
\hline BIA & Bulk inversion asymmetry \\
\hline $\mathrm{CH}$ & Crystal field split-off hole \\
\hline DFT & Density functional theory \\
\hline DLP & Degree of linear polarization \\
\hline DOS & Density of states \\
\hline DBR & Distributed Bragg reflector \\
\hline EEL & Edge emitting laser \\
\hline EFA & Envelope function approximation \\
\hline FBZ & First Brillouin zone \\
\hline $\mathrm{HH}$ & Heavy hole \\
\hline Laser & Light amplification by stimulated emission of radiation \\
\hline LED & Light emitting diode \\
\hline $\mathrm{LH}$ & Light hole \\
\hline NW & Nanowire \\
\hline $\mathrm{OC}$ & Optical confinement \\
\hline PWE & Plane wave expansion \\
\hline QC & Quantum confinement \\
\hline SOC & Spin-orbit coupling \\
\hline SO & Split-off hole \\
\hline VCSEL & Vertical cavity surface emitting laser \\
\hline VECSEL & Vertical external cavity surface emitting laser \\
\hline VLC & Vapor-Liquid-Crystal \\
\hline VLS & Vapor-Liquid-Solid \\
\hline WZ & Wurtzite \\
\hline $\mathrm{ZB}$ & Zinc-blende \\
\hline
\end{tabular}





\section{Summary}

1 Introduction.................................................... 19

1.1 Electronic structure of semiconductors . . . . . . . . . . . . . . . . . . . . 19

1.2 Spin lasers . . . . . . . . . . . . . . . . . . . . . . . 23

1.3 Polytypism in III-V zincb-blende/wurtzite materials . . . . . . . . . . . . . . . . 29

1.4 Spin-orbit coupling effects in wurtzite materials $\ldots \ldots \ldots \ldots$

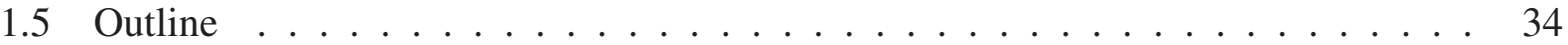

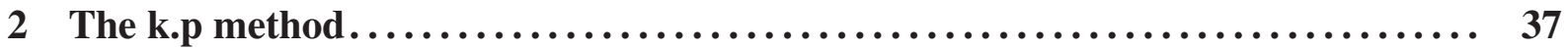

2.1 Bulk materials . . . . . . . . . . . . . . . . . . . 37

2.1 .1 The role of group theory $\ldots \ldots \ldots \ldots \ldots \ldots$

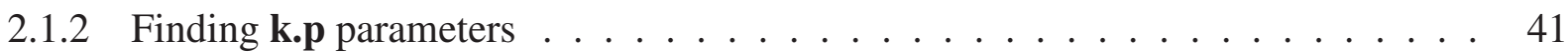

2.2 Quantum confined systems . . . . . . . . . . . . . . . . . . . . 43

3 Absorption and gain coefficients................................ 49

3.1 General formulation . . . . . . . . . . . . . . . . . . . . . . . . . 49

3.2 Spin-polarized gain $\ldots \ldots \ldots \ldots \ldots \ldots \ldots$

3.3 Simple analytical model $\ldots \ldots \ldots \ldots \ldots$. . . . . . . . . . . . 54

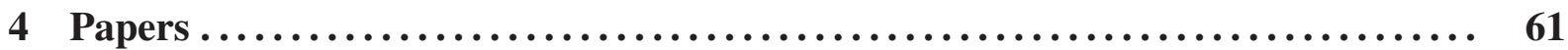

4.1 Toward high-frequency operation of spin lasers . . . . . . . . . . . . 62

4.2 Band structure calculations of InP wurtzite/zinc-blende quantum wells . . . . . . 77

4.3 Interband polarized absorption in InP polytypic superlattices $\ldots \ldots$. . . . . . 95

4.4 Realistic multiband $k \cdot p$ approach from $a b$ initio and spin-orbit coupling effects of InAs and InP in wurtzite phase . . . . . . . . . . . . . . . . 104

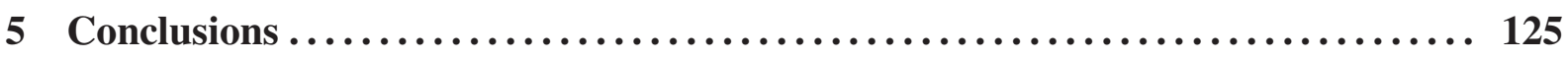

5.1 Future possibilities . . . . . . . . . . . . . . . 127

References..................................................... 129 



\section{Introduction}

\subsection{Electronic structure of semiconductors}

Analogous to the energy levels in atoms, the electronic (or band) structure expresses the allowed and forbidden energy states of electrons in a solid. After the great success of quantum mechanics in describing these atomic energy levels, physicists realized the potential, and requirement, of using the quantum theory to explain the physical behavior of electrons in solid state materials. For instance, neither the Drude-Lorentz model nor the Sommerfeld theory were able to fully explain the conductivity phenomena in metals.(1) Although Sommerfeld theory used the correct Fermi-Dirac statistics for electrons, the electronic energy was still obeying a free electron dispersion, which neglects the quantum mechanical role of the crystal lattice. It was in 1928 that Felix Bloch, the first student of Werner Heisenberg in Leipzig (Germany), investigated the quantum mechanical behavior of electrons subjected to a periodic crystal potential. He discovered that the electron wave function was modified to be a plane wave modulated by a periodic function, which is now called Bloch's theorem. By taking into account the ions of the crystal lattice, Bloch established the basic framework for the quantum mechanical approach to the band theory of crystalline solids, i. e., the theory that allows the calculation of the electronic structure in solid state materials.

Bloch's result was soon applied to understand the behavior of unexplained physical phenomena, for instance, the sign change in Hall conductivity that could not be predicted by the Sommerfeld model. Rudolf Peierls, another student of Heisenberg in Leipzig, explored the nonquadratic behavior between energy and crystal momentum to explain the positive Hall conductivity at the end of 1928*. It was also Peierls that established the concept of energy bands and bang gaps for electrons in crystals and also that unfilled states behave as positive charges with

\footnotetext{
* Nonparabolic behaviors in the band structure can lead to negative effective masses for electrons.
} 
positive effective mass, i. e., the holes. However, it was only in 1931 that Alan H. Wilson, working at Heisenberg's group in Leipzig at the time, classified crystals into metals, semiconductors and insulators by their energy bands. Metals have partially filled bands, while semiconductors and insulators have a completely filled band. The difference between semiconductors and insulators is the energy gap that separates the fully occupied band to the next empty one. In Fig. 1.1, we present the schematics of energy bands for the free electron model, a metal and a semiconductor/insulator. The filled energy band of the insulator does not allow the flow of any electric current. Semiconductors lie in between a metal (conducts electrical current) and an insulator (no conduction of electric current). This privileged place of semiconductors allows the on and off switch of electric current, an essential ingredient to sustain logic operations. In fact, the concept of energy bands combined with other experimental evidence led to the development of the transistor in 1947, the key element in modern electronics. This discovery of the transistor was recognized by the Nobel prize in physics of 1956 for William B. Shockley, Walter H. Brattain and John Bardeen.

(a) Free electron

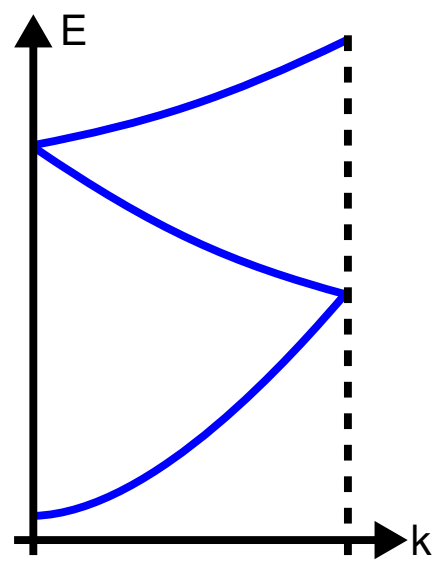

(b) Metal

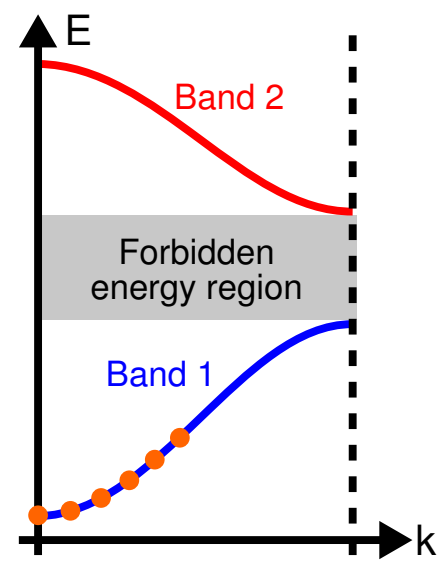

(c) Semiconductor or insulator

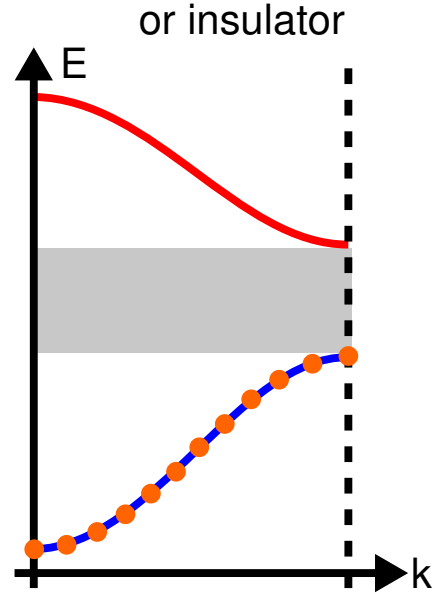

Figure 1.1 - Band structure for the (a) free electron model, (b) a metal and (c) a semiconductor or insulator. The energy bands in Figs. (b) and (c) are indicated in different colors and labeled, for simplicity, as bands 1 and 2. The filled circles indicate occupied states, therefore metals show a partially occupied band with empty states for electrons to access, while semiconductors and insulators have a fully occupied band. The next available band is separated by the energy gap, a forbidden region of energy.

Source: By the author. 
based on silicon and germanium, the understanding of the band structure of these materials was still imcomplete. For instance, it was only around the mid 1950s that reasonable band structures were obtained for $\mathrm{Si}$ and $\mathrm{Ge}$. The real problem at the time was not the theory itself, which comprises quantum mechanics, physical statistics and electromagnetism, but rather how to apply all these physical laws to the complex interactions of electrons with the crystal lattice and also with the other electrons. We emphasize that it is beyond the scope of this thesis to mention all the historical developments and approximations ${ }^{\dagger}$, however, it is important to note that one of the great achievements of solid state physics is the development of the band theory that allows the calculation, as accurately as possible, of the electronic structure of solid state materials such as semiconductors. Nowadays, the most common approaches to obtain the band structure of solids rely on: (i) ab initio, (ii) tight biding and (iii) k.p methods. Below, we address the main features of these different approaches.

$A b$ initio, or first principles, methods to calculate the electronic structure rely on the basic physical laws of quantum mechanics, statistical physics and electromagnetism applied to the specific atomic constituents of the semiconductor system, i. e., the atomic elements and their positions (crystal structure). It is also possible to provide experimental information, such as lattice constants and energy gaps, as input in order to increase the accuracy and reduce the computation time of the calculations. Ab initio methods can be based on the wave function description, such as Hartree-Fock, or on the electronic density, such as the density functional theory (DFT). The DFT approach is nowadays more common and widely used because of the reduced computational effort compared to the many-electrons wave function approach.(3) For solids in the bulk form, the ab initio theoretical framework is the most reliable technique to obtain the electronic structure, specially when combined with experimental data. On the other hand, the $a b$ initio approach has the disadvantage of being computationally demanding for systems with quantum confinement (QC), such as quantum wells, wires and dots, specially at the mesoscopic scale that requires a large number of atoms.

\footnotetext{
$\dagger$ The historical information presented up to this point was mostly obtained from the book Out of the Crystal Maze.(2) A very detailed survey on the roots of solid state physics that led to the development of band theory can be found in the first three chapters of this book along with the references for the original papers, mostly in German.
} 
The tight binding(4) approach is also atomistic, similar to $a b$ initio methods, and relies on the overlap parameters between atomic orbitals, also known as hopping parameters. Without the actual knowledge of these orbitals and the crystal potential, these hopping parameters are unknown variables and should be obtained from outside of the tight biding approach, usually from $a b$ initio. Although tight biding provides an intuitive picture of interacting atomic orbitals, the resulting Hamiltonian can be quite large depending on the number of orbitals and atoms required to describe the system. Nowadays, robust codes that combine $a b$ initio and tight binding using localized Wannier functions(5) are available to automatically extract the hopping parameters. The disadvantage of such approach is the large number of functions and hoping parameters, making it difficult for a physical analysis.

An alternative theoretical approach for band structure calculations of semiconductors is the k.p method(6), which is employed in this thesis. Unlike the atomistic character of ab initio and tight binding, the k.p method is a continuum approach with the smallest grid region being the primitive unit cell of the crystal structure ${ }^{\ddagger}$. Usually based on a perturbative approach(8-11) for the energy bands around high-symmetry points of the reciprocal space, the k.p Hamiltonian is constructed by using the periodic functions of the Bloch states as basis set. Regardless of the specific atomic orbital composition that comprises this periodic function, the important information is to which irreducible representation of the symmetry group it belongs ${ }^{\S}$. It should be noted that both k.p and tight biding methods rely on symmetry arguments of group theory to identify the nonzero coupling parameters and, therefore, require an external method to assign values to these parameters. The great advantage of using k.p is to model quantum confined systems using the envelope function approximation(14-19), by "simply" making the substitution $\vec{k} \rightarrow-i \vec{\nabla}$.

The k.p method has been successfully applied over the years to investigate a large range of physical phenomena in semiconductors systems. It is worth mentioning that k.p has been

\footnotetext{
$\ddagger$ Recently there was a suggestion for an atomistic k.p approach from Pryor and Pistol(7) that reduces the space grid to atomic positions, recovering the tight binding approach.

$\S$ An irreducible representation contains the information of how functions behave under the symmetry operations of the group. $(12,13)$
} 
widely used in the research field of semiconductor spintronics ${ }^{\top}(20,21)$ to investigate spinorbit coupling (SOC) effects in quantum wells(22-27), optical spin injection(28-31) and spindependent optical properties of spin lasers(32, 33), among others. Moreover, the k.p method has been recently revived for studies of unconventional semiconductor systems such as topological insulators(34-37), zinc-blende(ZB)/wurtzite(WZ) polytypic nanostructures(38-40) and two dimensional materials.(41-43) Particularly in this thesis, we investigated three different subjects using the band structure obtained from k.p models: (i) spin lasers, (ii) ZB/WZ polytypism in III-V semiconductors and (iii) SOC effects in InAs and InP WZ materials.

\subsection{Spin lasers}

The laser, abbreviation of light amplification by stimulated emission of radiation, is a unique light source capable of producing coherent and focused light beams.(44) Although the theoretical foundation for the stimulated emission was proposed by Einstein in 1917, it was only in 1960 that the first operational laser was realized, using a ruby crystal ${ }^{\|}$. In these early years, lasers seemed to be a solution without a problem. However, as time passed by, lasers become deeply present in our society. Nowadays, semiconductor lasers, or simply laser diodes, are the most common type of lasers that permeate our daily lives. They have small power consumption that has been reduced over the years by using quantum confined structures in the active region.(46) The large variety of existing semiconductor materials allows laser diodes to have a broad optical emission range, from ultraviolet to infrared range of the electromagnetic spectrum. Furthermore, laser diodes are used as the light source for fiber optics communication, capable of transmitting huge amounts of information (data) over large distances**. We can further mention a few applications based on semiconductor lasers: optical media players (CD/DVD/Blu-ray), computer mouse, barcode readers, laser pointers, etc.

Irrespective of the application, all semiconductor lasers have three important components:

\footnotetext{
T Spintronics, acronym for spin transport electronics, is a multidisciplinary research field in condensed matter that investigates the spin degree of freedom of the electrons and their interaction with the surrounding media.

$\|$ A historical description of Nobel prizes related to lasers can be found in the reference.(45)

** Binary information is encoded with low and high intensity light pulses, which is realized by modulating the current injection.(47)
} 
(i) the active (gain) region, where electron-hole recombination takes place, (ii) the resonant cavity, sandwiched by high reflective mirrors responsible for confining the laser light, and (iii) the pump mechanism, responsible for injecting carriers in the active region (either electrically or optically). Once these injected carriers generate light by radiative recombination, this light is trapped inside the cavity providing the feedback that allows further recombination. For a sufficiently large injection, above the so-called lasing threshold, the laser operation is achieved emitting an intense focused beam of coherent photons. This light coherence is locked by the cavity, which defines the wavelength of emitted light. Without the resonant cavity, the emitted light by carrier recombination would not provide feedback for laser operation. In fact, LEDs (light emitting diodes) can be understood as lasers without a resonant cavity, leading to an incoherent emission of light with small intensity. Considering the active region, laser operation is achieved when the gain coefficient ${ }^{\dagger \dagger}$ is larger than the gain threshold (the losses in the cavity), i. e., more photons are created instead of absorbed. The values for gain coefficient depend on several factors, for instance, the material used in the active region, the temperature, the photon energy, etc.

Similar to conventional lasers, spin lasers also share the same components discussed above. The main difference lies in the pumping mechanism, which, for spin lasers, allows the injection of spin-polarized carriers in the active region. Because of the conservation of the total angular momentum during electron-hole recombination $(20,48)$, the output light in spin lasers is circularly polarized with a preferred helicity, depending on the net spin imbalance. This spin dependent light-matter interaction that happens in spin lasers offer possible opportunities for spin dependent controllable devices operating at room temperature.(49) However, spin lasers are not only of interest to the emergent research field of spintronics, but also to the laser community. Experimentally, it is already demonstrated that the nonzero spin polarization in the active region provides enhanced steady-state operation features in spin lasers compared to their conventional (spin-unpolarized) counterparts. For instance, a reduced amount of injected carriers is needed to achieve lasing threshold(50-52), an effect known as threshold reduction. Moreover, circularly

\footnotetext{
$\dagger \dagger$ This gain coefficient is defined as the ratio between the number of photons emitted per second per unit volume and the number of injected photons per second per unit area, having a dimension of 1/length. It also determines the decay or growth of the electric field intensity.(44)
} 
polarized light with high degree of polarization is emitted even for a small net value of injected spin polarized carriers $(53,54)$, providing a robust spin-filtering mechanism.

It is helpful to compare the operation of conventional and spin lasers using the bucket model analogy.(33, 55, 56) For the conventional laser depicted in Fig. 1.2(a), spin up and spin down carriers are injected in the system with the same amount, represented by the water coming out of the single faucet. Consequently, the light output, represented by the water flowing out of the bucket, has an equal combination of circular helicities. Under small injection, water leaks out of the bucket through the small apertures, representing the no lasing regime (and possible losses). Increasing the injection, the water level can reach the large opening and achieve the lasing regime. In Fig. 1.2(b) we depict the spin laser case, dividing the bucket in two parts that are filled separately by different faucets, which indicate the carrier injection with different spins. For instance, hot (cold) water indicates spin up (down) carriers injection in the system. Due to this uneven flow of water into the bucket, it is necessary to (approximately) fill half of the bucket to reach the large opening. In other words, the lasing regime is achieved by adding a smaller amount of water as compared to the convention bucket case. This reduced amount of water injection to achieve lasing is the aforementioned threshold reduction phenomena in spin lasers.(50-52) Furthermore, the water that gushes out in Fig. 1.2(b) is mainly hot water, which can be viewed as the consequence of spin-filtering $(53,54)$, i. e., the spin polarization of emitted photons greatly exceeds the spin polarization of injected carriers. Even though there is a small difference between hot and cold water added to the bucket, the output flow of water is reached first by one kind. In Figs. 1.2(c) and (d) we show graphically the light output as function of the injection. Lasing operation is achieved for the conventional case if the input is greater than the threshold $T$. In the spin laser case, each light polarization achieves lasing for different injections, $T_{a}$ and $T_{b}$. Spin-filtering regime is achieved when $\left|T_{a}-T_{b}\right|>0$ and threshold reduction when $T_{a}<T$ (assuming $T_{b}>T_{a}$ ).

In real spin laser devices, the most common used geometry is called VCSEL(57), acronym for vertical cavity surface emitting laser. We show in Fig. 1.3(a) one possibility for VCSEL design. The three main components of a laser, previously discussed, are also present here: (i) 

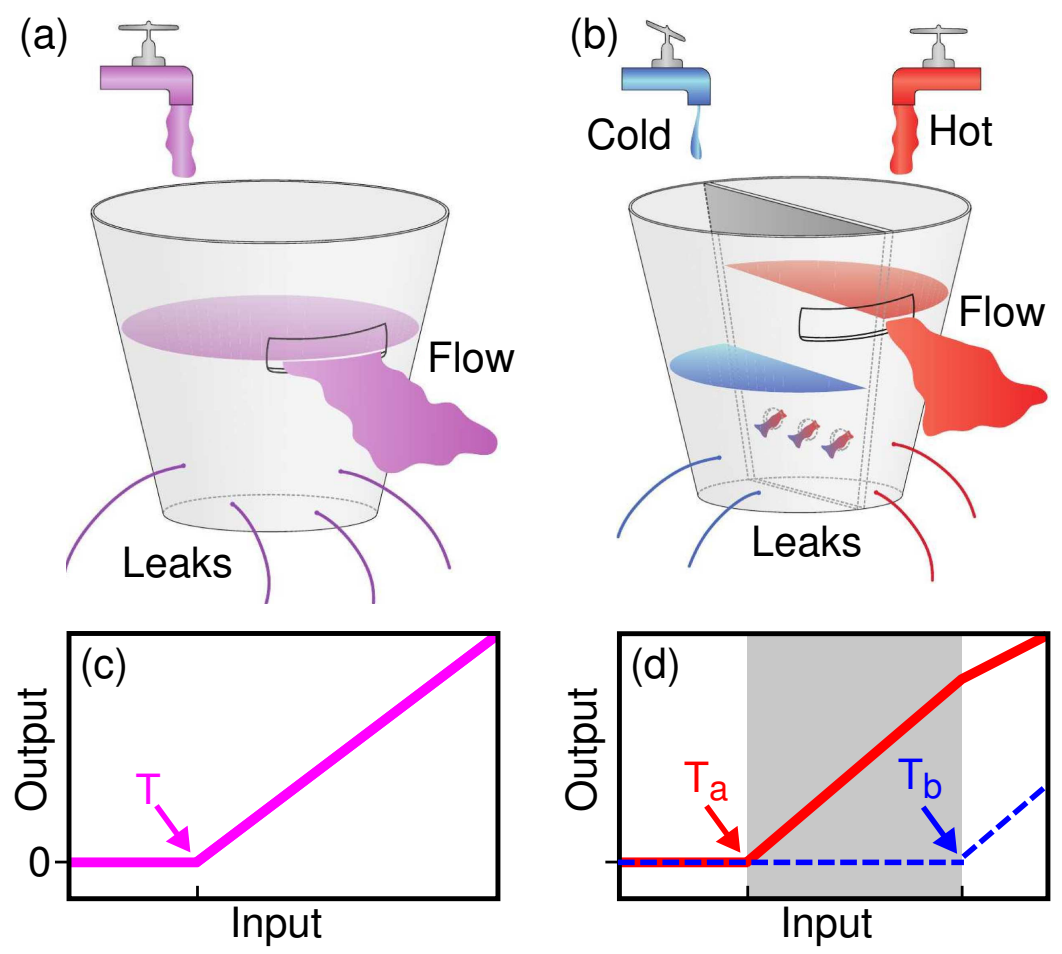

Figure 1.2 - The bucket model analogy for (a) conventional and (b) spin lasers. Water flowing into the buckets represent the carrier injection and water flowing out of the bucket indicate light emission. Lasing threshold is achieved when the water level reaches the large opening of the bucket. The leaks indicate the light emitted in the no lasing regime. Small holes between the two sides of the bucket in Fig. (b) represent the mixing of spin populations due to spin relaxation. Graphical description of input vs output (injection vs emission) for (c) conventional and (d) spin lasers. The lasing threshold is indicated by the point $T$, for conventional lasers, and by the points $T_{a}$ and $T_{b}$ for spin lasers. The dark area shows the spin-filtering regime between $T_{a}$ and $T_{b}$.

Source: (a-b) Adapted from ZUTIC, I.; FARIA JUNIOR, P. E.(56); (c-d) By the author

the quantum well active region (ii) the resonant cavity (Cav) sandwiched by top and bottom distributed Bragg reflectors (tDBR and bDBR) and (iii) the pump/injection mechanism represented by top and bottom contacts ( $\mathrm{tC}$ and $\mathrm{bC}$ ). Usually, the ARs of these geometries are realized with quantum wells or quantum dots of III-V semiconductor compounds. $(52-54,58-61)$ The DBRs are high reflective mirrors $(\geq 99.5 \%)$ having a sequence of alternating layers of semiconductor materials with high and small refractive indices. Tuning the thickness of these layers by one quarter of the material wavelength allows the peak reflectivity of DBRs to increase by adding more pairs. For comparison, the total length of the VCSEL is $\mathrm{L}_{\mathrm{V}} \sim 10 \mu \mathrm{m}$ while the size of the active region is typically $\mathrm{L}_{\mathrm{W}}=10-100 \mathrm{~nm}$, according to the number of quantum wells considered. The size of the resonant cavity is connected to the wavelength of the output light. 
For the resonant condition of the wavelength $\lambda$, we have

$$
m \lambda=2 n_{r} \mathrm{~L}_{\mathrm{C}}
$$

with $n_{r}$ being the refractive index and $m$ the mode profile of the electric field. Such VCSEL geometries have several advantages over the edge emitting laser (EEL) geometry ${ }^{\ddagger \ddagger}$, for instance, small lasing threshold, higher efficiency at low power, small divergence of the output light and reduced dimensions.(57) It is also worth mentioning the vertical external cavity surface emitting laser (VECSEL) geometry, used in recent spin laser experiments.(62-64) In this geometry, the tDBR of the VCSEL is replaced by an external reflective mirror. The advantage of such approach is that the cavity length becomes an additional degree of freedom for the system, enabling the control of the resonant frequency of photons without the need to change the growth design of the device.

Although steady-state properties of spin lasers seem promising, the most fruitful opportunities may lie in their dynamic operation. It has already been theoretically predicted that either intensity or polarization modulation of injected current in spin lasers provides an increased bandwidth(65) for the output light intensity $§ \S$ This enhancement in the bandwidth was also experimentally demonstrated for current modulation in quantum dot spin VCSELs.(53) Furthermore, there are experimental efforts on spin lasers showing that light polarization dynamics operates at a higher frequency than the output intensity $(60,61)$ and that these oscillations can be controlled.(67) These oscillations in the light polarization are possible because of the birefringence $\boldsymbol{\Phi}^{\top}$ in the system that can be increased by external mechanical force, which ultimately leads to higher oscillation frequencies.(68) We can understand the effect of this induced birefringence on the VCSEL from Fig. 1.3(b). The application of an external mechanical force(68) to the system creates an anisotropic strain field that propagates throughout the VCSEL structure. Moving away from the substrate this strain field decays and, given the lengths of the whole VCSEL and the cavity, we can assume a small and constant strain along the active region.

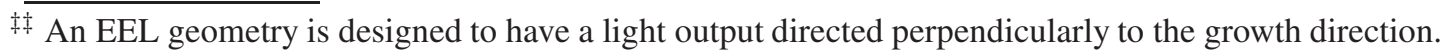

$\S \S$ The bandwidth, with dimension of $\mathrm{Hz}$, is defined by the frequency response curve of the laser, which shows how the light output intensity changes due to current modulation. $(44,66)$

9ा Simply, birefringence is the anisotropy of the index of refraction leading to different polarization modes (see eq. (1.1) for clarity). For the operation of conventional lasers, birefringence values are considered detrimental.(66) 

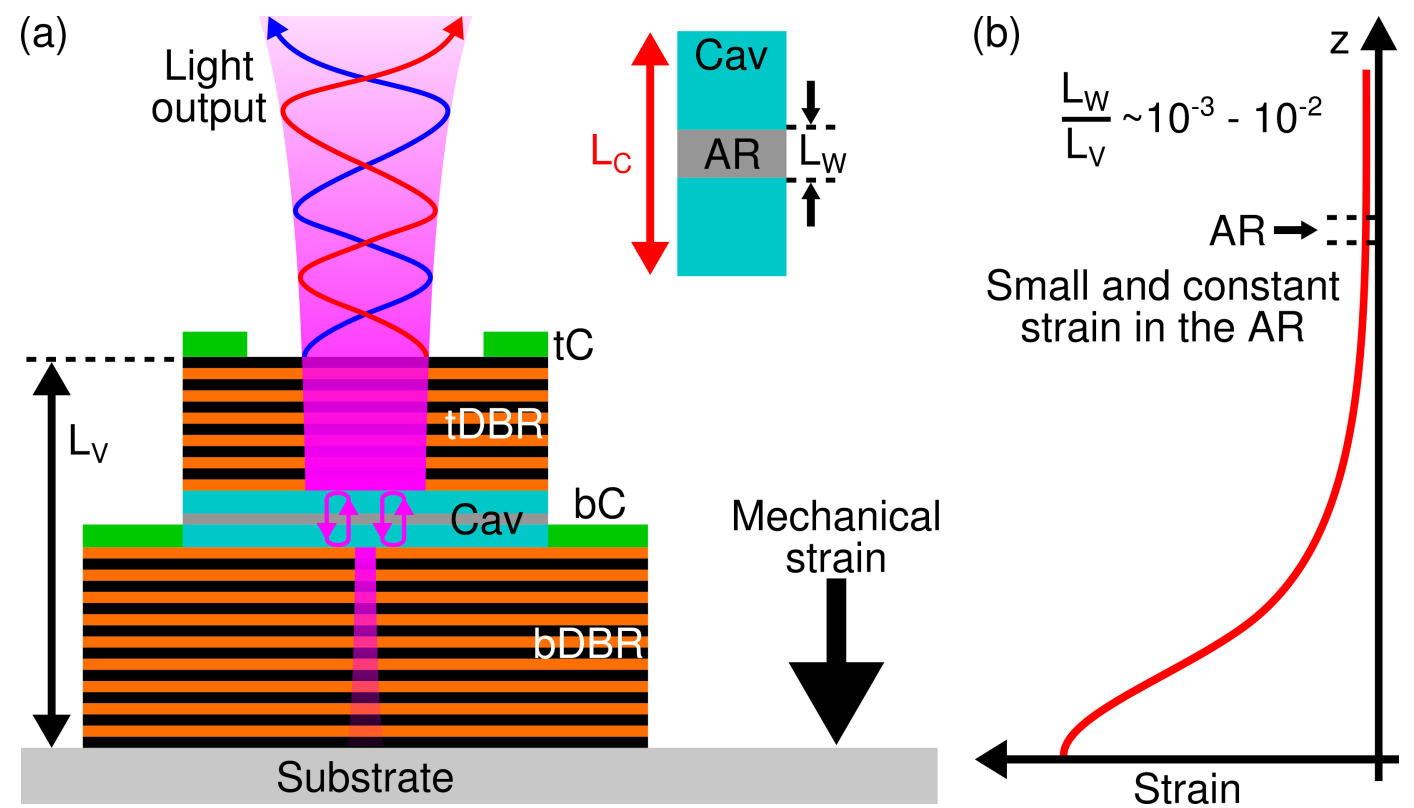

Figure 1.3 - (a) Possible design of a VCSEL, including the top (tDBR) and bottom (bDBR) DBRs, the top (tC) and bottom (bC) contacts and the resonant cavity (Cav). The arrows inside the cavity indicate the light feedback of trapped photons. The output light is emitted upwards and the twisted arrows indicate positive and negative helicities for light polarization. Since the bDBR is not a perfect mirror, light can also be emitted downwards. In the inset, the cavity and the active region (AR) are shown in detail. The lengths of the whole VCSEL, the cavity and the active region are indicated by $\mathrm{L}_{\mathrm{V}}, \mathrm{L}_{\mathrm{C}}$ and $\mathrm{L}_{\mathrm{W}}$, respectively, We also show a possible scheme for applied mechanical strain at the substrate. (b) The effect of mechanical strain along the VCSEL. The strain field propagates upwards and loses its amplitude. In the scale of the active region, compared to the whole VCSEL, it is reasonable to imagine a small and constant strain.

Source: By the author.

A conventional theoretical framework to investigate spin lasers is provided by the rate equations $(50,51,55,65,69-71)$, for steady-state and dynamic operation, and spin-flip model(60, $61,67,72,73)$, for the spin-induced dynamics in light polarization. Although these approaches are very helpful to understand experiments by fitting the unknown parameters, they do not capture the complex behavior of conduction and valence bands. For instance, rate equations predict only threshold reduction by increasing spin polarization regardless of the cavity design and spin flip models make no distinction between the physical origins of birefringence, either coming from active region or DBR. Since semiconductor lasers are bipolar devices, a simultaneous description of electrons and holes is crucial, specially because of the different types of valence subbands that provide different selection rules for light polarization.(20) Therefore, a microscopic treatment of spin lasers becomes necessary in order to elucidate the physics that 
is not present in the conventional framework. Up to date, there are only two papers in the literature based on band structure calculations: (i) the study of Holub and Jonker(32), using a $6 \times 6$ k.p model to investigate the threshold reduction from the gain peak (not suitable for VCSEL devices), and (ii) our study [Faria Junior et al.(33)], using a $8 \times 8$ k.p model to investigate spin-dependent gain properties for VCSEL devices considering different cavity designs and also considered uniaxial strain to investigate the effects of birefringence from the active region and DBR. Furthermore, results from microscopic models could be used as inputs for rate equations and spin flip models.

\subsection{Polytypism in III-V zincb-blende/wurtzite materials}

Among the diverse set of semiconductor materials, binary compounds that combine elements of group III (typically Al, Ga and In) and group V (typically N, P, As and Sb) of the periodic table have great technological interest.(74) In the bulk form, the arsenides, phosphides and antimonides have $\mathrm{ZB}$ crystal structure as the most stable phase while nitrides crystallize in $\mathrm{WZ}$ phase. The atomic arrangement of ZB structure shows a cubic symmetry and can be described by a face centered cubic lattice with two atoms in its basis. On the other hand, WZ structure shows a hexagonal symmetry and 4 atoms in the primitive unit cell. In order to understand these two different crystal structures, we compare them in Fig. 1.4. In Figs. 1.4(a) and 1.4(b) we show the conventional and primitive unit cells of $\mathrm{ZB}$, characterized by the lattice constant $a$. In Figs. 1.4(d) and 1.4(e) we show the conventional and primitive unit cells of WZ, characterized by the lattice constants $a$ and $c$. The FBZs are depicted in Figs. 1.4(c) and 1.4(f), for ZB and WZ, respectively.

Although the atomic arrangement of ZB and WZ crystal structures seems completely different, these crystal phases show differences in the internal energies of only $\sim 20 \mathrm{meV} /$ atom.(75) These small values can be understood if we look at ZB along [111] direction, i. e., parallel to the diagonal of the cubic conventional cell [Fig. 1.4(a)]. In reciprocal space, [111] direction is equivalent to the line from $\Gamma$ to $L$ point in the FBZ [Fig. 1.4(c)]. In this direction, ZB can be described by a hexagonal stacking sequence of $\mathrm{ABCABC}$ layers, with each letter representing 
(a)

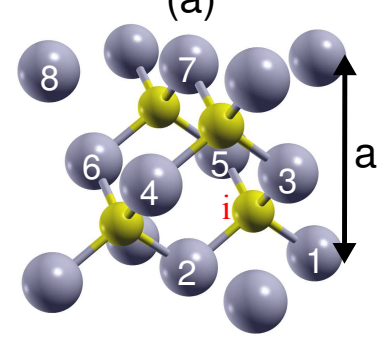

(d)

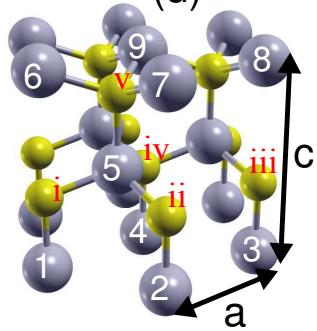

(b)
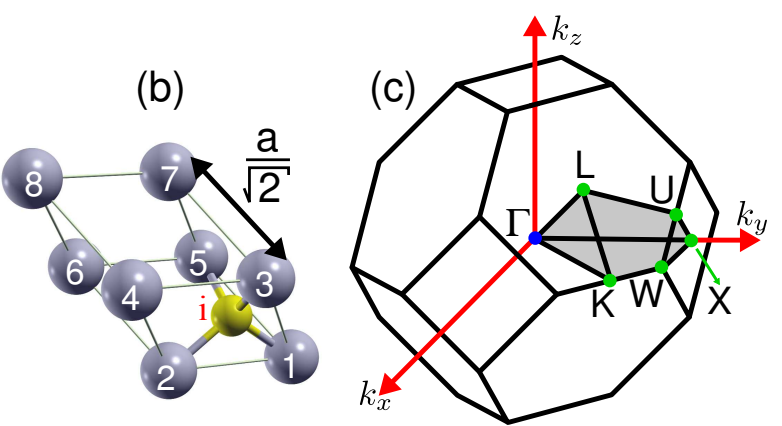

(e)

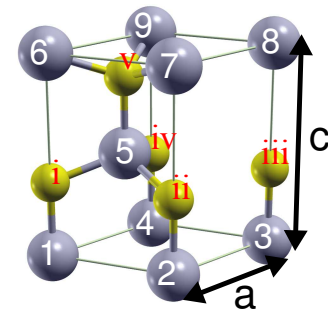

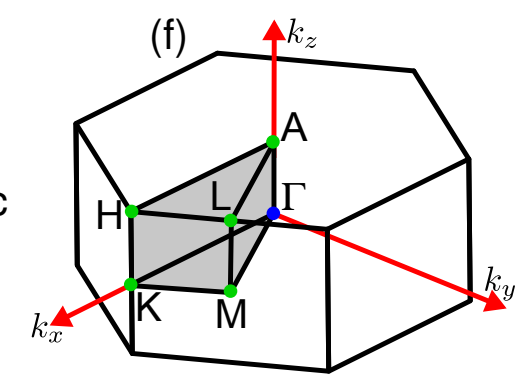

Figure 1.4 - (a) ZB conventional unit cell. (b) ZB primitive unit cell. (c) ZB FBZ. (d) WZ conventional unit cell. (e) WZ primitive unit cell. (f) WZ FBZ. The atoms are numbered to simplify the connection between conventional and primitive unit cells. The arabic numbering indicate atoms of type 1 while roman numbering indicate atoms of type 2 . In the FBZs, the shaded region is the irreducible wedge created by the high-symmetry points.

Source: By the author.

a bilayer of atoms. On the other hand, WZ along [0001] direction has ABAB stacking sequence (see for instance Fig. 1 in Sec. 4.2 for comparison). Because of this small energy difference, it is possible to observe the appearance of both crystal phases in the same system depending on the growth conditions used. This mixture of both ZB and WZ crystal phases in the same structure is called polytypism. Motivated by the polytypic appearance of other binary semiconductor compounds (e. g., SiC, CdS, CdSe), the theoretical investigation of III-V ZB/WZ interfaces was performed in the early 1990s. $(75,76)$ Besides the band offsets created by the polytypic interface, the paper of Murayama and Nakayama(76) showed the connection of different energy bands at the interface from a group theory point of view (connection of irreducible representations). Since ZB has half of the atoms of WZ unit cell, the authors showed that additional energy bands from ZB $L$ point are also folded to $\Gamma$ point to correctly match the number of energy bands in WZ $\Gamma$ point. This connection concept of energy bands is crucial to theoretically investigate $\mathrm{ZB} / \mathrm{WZ}$ polytypism.

Despite the theoretical predictions, it was not until the late 2000s that the controlled growth 
of important III-V semiconductor compounds was achieved in WZ phase for GaAs, GaP, InAs and InP.(77-83) The most common approach of growing these nanostructures, called VaporLiquid-Crystal (VLC) or Vapor-Liquid-Solid (VLS)(84), requires a metal particle seed deposited in the substrate, usually Ag (silver) or Au (gold)(82), leading to a radial dimension of the resulting nanowire (NW) with roughly the same size of the metal droplet. For this fixed diameter imposed by the metal particle seed, other growth parameters can be controlled, for instance, III/V ratio and temperature, to achieve either pure phase $\mathrm{NWs}(85,86)$ or polytypic superlattices with well defined $\mathrm{ZB} / \mathrm{WZ}$ regions.(81, 83, 87, 88) It was also shown recently that the ZB/WZ phase alternation is not only limited to the axial direction of the NW (parallel to NW axis) but it can also appear when NWs are radially merged (perpendicular to NW axis).(89) Furthermore, a recent paper discusses the possibility of other polytypes in III-V semiconductor NWs.(90) It was shown that besides ZB and WZ crystal structures, two additional hexagonal stacking sequences can also appear in the NW: $4 \mathrm{H}(\mathrm{ABCBABCB})$ and $6 \mathrm{H}(\mathrm{ABCACBABCACB})$. However, under specific growth conditions, it is possible to isolate this polytype attainability to ZB/WZ mixing only.

In some applications this phase mixing is considered detrimental to the physical properties such as electronic transport in InAs NWs(91), however these new forms of band offsets can also be used to control and improve optical properties. For instance, the lasing operation at room temperature was demonstrated for a ZB/WZ InP nanolaser integrated on Si substrate.(92) The type-II polytypic band alignment of InP and the different widths of ZB and WZ segments are responsible for a large range of wavelengths. Furthermore, pure WZ and ZB InP NWs show different light polarization properties(79), i. e., ZB (WZ) NWs have predominant light polarization parallel (perpendicular) to the NW axis. Combining ZB and WZ crystal phases in the same NW can be used to control the light polarization.(93) In addition, from the measured light polarization it would be possible to determine the amount of $\mathrm{WZ}$ and $\mathrm{ZB}$ phase mixing inside the system.

These advances in growth techniques and experimental characterization of polytypic and pure WZ nanostructures also received theoretical attention. Focusing on these new possibili- 
ties of III-V WZ materials, several ab initio studies(94-99) calculated the bulk band structure throughout the FBZ, providing band gaps, energy splittings and lattice constants. In the paper of De and Pryor(95), they systematically investigated the bulk band structure of III-V (with $\mathrm{Al}, \mathrm{Ga}$ and In for group III and P, As and Sb for group V) semiconductors in WZ phase and provided useful data such as effective masses, energy splittings and spin splitting parameters close to the $\Gamma$-point that can be considered in simple two-band effective models. Such simple approaches have the disadvantage of being reliable only in a very restricted region around the $\Gamma$-point, consequently limiting the possible physical phenomena that can be investigated. For instance, optical transitions require at least a dipole coupling term between conduction and valence bands. In order to provide a realistic description for these new possibilities of III-V WZ materials, effective multiband k.p Hamiltonians with reliable parameter sets are desirable options. These multiband approaches can also be easily coupled with the envelope function approximation to investigate QC and proximity effects in nanostructures. Furthermore, since $\mathrm{ZB} / \mathrm{WZ}$ polytypism is already demonstrated experimentally and ZB III-V materials are well established(100), the model we present in this thesis for ZB/WZ polytypic InP quantum wells and superlattices $(38,39)$ can be applied to other semiconductor compounds, offering a reliable theoretical approach to understand and predict experiments on ZB/WZ polytypic systems.

\subsection{Spin-orbit coupling effects in wurtzite materials}

Besides the interesting possibility of polytypic nanostructures with non-nitride III-V WZ semiconductors, pure phase NWs have attracted great attention, for instance, regarding spinrelated properties. Recent experimental setups using semiconductor nanowires and proximity induced s-wave superconductivity provide a robust platform to study the quasiparticle manifestations of Majorana fermions(101-105), particles that are their own antiparticles. Typically, the nanowires used in these experiments are grown with InSb in ZB phase $(102,103)$ and InAs in WZ phase $(104,105)$, i. e., materials with large intrinsic SOC, one of the key ingredients required to accommodate the Majorana states. From a theoretical perspective, these hybrid semiconductor/superconductor setup cannot be investigated by ab initio methods because of the large 
size of the whole system and, therefore, effective Hamiltonians should be considered. Furthermore, despite the interest in optical properties of pure WZ phase NWs of $\operatorname{InP}(79,92,106-108)$, there are also recent magneto-optical measurements(109) that allow the characterization of conduction and valence band effective gyromagnetic factors, or simply g-factor, a physical quantity that also is affected by the intrinsic SOC.(110-112) Since pure phase NWs with large diameter have small QC effects and can be considered as bulk materials, they can be easily investigated using bulk multiband Hamiltonians. Therefore, effective Hamiltonians that include the intrinsic SOC effects are essential theoretical tools.

For $\mathrm{WZ}$ and $\mathrm{ZB}$ materials that lack inversion symmetry, known as BIA (bulk inversion asymmetry), one of the manifestations of this intrinsic SOC is the spin splitting of energy bands at a general point of the FBZ (typically not a high-symmetry point). In the ZB case, conduction band electrons close to $\Gamma$-point show a cubic dispersion $\left(\propto k^{3}\right)$ and this BIA SOC is called Dresselhaus(10). On the other hand, electrons in WZ conduction band close to $\Gamma$-point show a linear dispersion $(\propto k)$ and was first derived by Rashba and Sheka(113). This linear splitting SOC term was also found by Bychkov and Rashba(114) in quasi two dimensional systems and that it could be controlled by external gate voltage, therefore providing a tunable SOC effect which is now know as Rashba SOC ${ }^{* * *}$. Based on the Rashba SOC, Datta and Das suggested the concept of spin field-effect transistor in 1990(116), a key study to the field of spintronics.(20) Even though this $k$-dependence close to $\Gamma$ point is well understood and supply a fertile playground for physics, the rigorous treatment of these SOC effects in multiband Hamiltonians is not often considered. For instance, in ZB semiconductors the $14 \times 14$ k.p Hamiltonian $(21,110,117)$ is often considered to obtain the spin splittings effects ${ }^{\dagger \dagger \dagger}$ instead of including the relativist $k$-dependent SOC terms in the conventional $8 \times 8$ k.p Hamiltonian.

Similarly to ZB, in WZ III-V semiconductors the $k$-dependent SOC effects are usually neglected(118-120) but in WZ there is no formulation to emulate the BIA splittings, specially for these unconventional III-V WZ materials. The situation gets worse because WZ is less symmetric than ZB and the Hamiltonians require more parameters, which makes the analytical

\footnotetext{
*** For a more detailed discussion of the Rashba SOC effect, please look at the paper (115) and references therein.

${ }^{\dagger \dagger}$ The spin splittings arise from the indirect coupling between $\vec{k} \cdot \vec{p}$ terms and the $k$-independent off-diagonal SOC terms.
} 
of parameters an impossible task. Therefore, a numerical approach to extract the k.p parameters has to be employed.(120-127) In this thesis, we provide for the first time the parameter sets for InAs and InP in WZ phase including the relativist $k$-dependent SOC terms in $8 \times 8$ k.p Hamiltonian to correctly model the BIA splittings.(127) Our Hamiltonian is numerically fitted to $a b$ initio band structure in multiple directions of the FBZ simultaneously. Besides the band structure fitting, we systematically checked spin splitting and spin texture properties to be consistent. With new Hamiltonians and parameters for these unconventional III-V WZ materials, the possibilities of band gap engineering with either pure or mixed crystal phases are drastically increased, allowing the scientific community to understand and predict novel physical phenomena in III-V semiconductor NWs.

\subsection{Outline}

While several studies focus only on applying previously published k.p Hamiltonians, this thesis has a two-fold effort: not only apply but also develop k.p models to investigate spin and optical properties in semiconductor systems and materials. In chapter 2, we describe the k.p theory for bulk and quantum confined systems and discuss the intimate connection between the k.p model and group theory. We also discuss the different approaches used in this thesis to obtain k.p parameters. In order to investigate optical properties, we introduce the absorption and gain coefficients in chapter 3. We also discuss the important considerations used in the spin-polarized gain framework. Before analyzing the optical properties of complicated semiconductor systems, we investigate the absorption, gain and spin-polarized gain coefficients using a fully analytical two-band model. Under this fully analytical approach, our analysis of gain and spin-polarized gain coefficients elucidates the behavior of conventional and spin lasers from the microscopic point of view. All the semiconductor compounds we investigate in this thesis belong to the III-V group and have either ZB or WZ crystal structures (see Fig. 1.4).

In section 4.1, we investigate the gain properties of the active regions of spin lasers. Before our approach, only one paper in the literature focused on the microscopic description of such systems(32), using a $6 \times 6$ k.p model and calculating threshold reduction for the gain peak, 
therefore not suitable for VCSEL operation. In our study, we focus on the spin-dependent gain properties of a GaAs quantum well with $\mathrm{Al}_{0.3} \mathrm{Ga}_{0.7}$ As barriers, a typical active region found in many commercial VCSELs. Based on band structure calculations using the conventional $8 \times 8$ k.p model for ZB materials, we investigate the effects of carrier density, spin polarization of electrons and different cavity designs in the gain coefficient. We elucidate the mechanism of gain asymmetry (different gain coefficients for $S^{+}$and $S^{-}$light polarization) by showing the interplay of injected spin-polarized electrons and the intrinsic selection rules of the calculated band structure. The steady-state features of spin-filtering and threshold reduction are also discussed. Considering a strain-induced birefringence, we use the microscopic model for birefringence coefficient by Mulet and Balle(73) to predict values above $200 \mathrm{GHz}$. These large values are nearly independent from the spin polarization of injected electrons, therefore confirming the in-plane anisotropy characteristic of birefringence. Recent experiments in similar VCSELs showed values up to $250 \mathrm{GHz}$ for the birefringence coefficient.(128) The results described in this section are published in the reference.(33)

Sections 4.2 and 4.3 are dedicated to ZB/WZ polytypism in InP quantum wells and superlattices, published in the papers. $(38,39)$ Specifically, in section 4.2 we focus on the development of the polytypic $6 \times 6 \mathrm{k} \cdot \mathbf{p}$ model to calculate $\mathrm{ZB}$ and $\mathrm{WZ}$ homostructures (the prefix homo is used to indicate same compound in the structure). We apply the polytypic model to calculate the band structure of a ZB/WZ quantum well considering quantum confinement, strain and polarization effects. For InP, the type-II band alignment and the polarization fields create spatial separation of electrons and holes, a feature that is present in our model. In section 4.3, we expand the $6 \times 6$ model to include the linear coupling between conduction and valence bands. This coupling is responsible for the optical selection rules in the system, dictating the input/output light polarizations. Considering InP polytypic superlattices we investigate the effect of crystal phase mixing and QC in the polarized absorption coefficient. We also include the optical confinement (OC) factor to take into account the dielectric mismatch at the interface. Our results for the bulk case including the OC are in good agreement with polarized photoluminescence measurements in pure phase NWs.(79) For the small QC case, i. e., large ZB and WZ regions, and small ZB composition, our results explain the polarized photoluminescence in polytypic 
NWs.(106) We found that the degree of linear polarization (DLP) in the polytypic system is sensitive to the crystal phase mixing and QC, allowing the possibility of light polarization engineering.(93)

Finally, in section 4.4 we investigate the band structure and SOC effects of bulk InAs and InP in WZ phase, published in the paper.(127) Based on the simple group approach, we present a robust $8 \times 8$ k.p Hamiltonian including the $k$-dependent SOC term, usually neglected in the literature.(118-120) We found reliable parameter sets by numerical fittings to ab initio band structures from WIEN2k(129), systematically checking spin splittings and spin texture features. The BIA of WZ structure is responsible for intricate behaviors in the spin splitting, i. e., linear, cubic, maxima values and crossings. Due to the large SOC in InAs, the valence bands LH (light hole) and $\mathrm{CH}$ (crystal-field split-off hole) have reverse ordering, i. e., CH is above LH. Thus, from top to bottom, the InAs valence band ordering is $\mathrm{HH}$ (heavy hole)-CH-LH while InP shows HH-LH-CH ordering. Despite the band ordering, we found a Rashba-like spin texture(20) with either clockwise or counter clockwise orientation for the spin splitting branches. Based on the $8 \times 8$ Hamiltonian, we calculate the density of states (DOS) and provide the carrier density as function of the Fermi energy. We also present independent formulations for conduction and valence bands, however, we emphasize that the best match to ab initio calculations is obtained with the $8 \times 8$ Hamiltonian. The robust theoretical framework we provide here can be directly applied to investigate InAs and InP based nanostructures and their related physical properties. 


\section{The k.p method}

The mathematical description of interacting electrons in a solid state system is a complex problem that, in reality, is impossible to be solved directly because of the large number of particles involved $\left(10^{22}-10^{23}\right.$ atoms $\left./ \mathrm{cm}^{3}\right)$ and, therefore, approximations are required. Under such approximations*, it is possible to obtain a single-particle description that replaces the electronelectron and electron-nuclei interaction by an effective potential. In order to find this potential and in fact solve the Schrödinger equation self-consistently, ab initio (or first principles) methods have to be employed. Alternatively, even without knowing the periodic potential and the wave functions, we can extract physical properties of crystals using the k.p method. In this chapter, we address the formalism of the k.p method for bulk materials and quantum confined systems.

\subsection{Bulk materials}

A bulk material is a crystalline solid with uniform properties throughout its whole dimension, that can be described by a periodic arrangement of unit cells in all directions. In order to develop the k.p method for this case, let us start with the single-particle Hamiltonian, including SOC, given by

$$
\left\{\frac{p^{2}}{2 m_{0}}+V(\vec{r})+\frac{\hbar}{4 m_{0}^{2} c^{2}}[\vec{\nabla} V(\vec{r}) \times \vec{p}] \cdot \vec{\sigma}\right\} \psi(\vec{r})=E \psi(\vec{r})
$$

Regardless of the functional form, the effective potential is periodic in the crystal lattice, $V(\vec{r})=V(\vec{r}+\vec{R})$, and therefore the Bloch's theorem can be used $\psi_{n, \vec{k}}(\vec{r})=e^{i \vec{k} \cdot \vec{r}} u_{n, \vec{k}}(\vec{r})$. The functions $u_{n, \vec{k}}(\vec{r})$ are also periodic in the Bravais lattice of the crystal, i. e., $u_{n, \vec{k}}(\vec{r})=u_{n, \vec{k}}(\vec{r}+\vec{R})$.

\footnotetext{
* A well organized summary of the important approximations used to arrive at the single-particle description can be found in section 2.2 of the book Fundamentals of Semiconductor Physics and Devices, by R. Enderlein and N. J. M. Horing.(130)
} 
We explicitly indicate the energy band label, $n$, and the wave vector $\vec{k}$, that lies within the FBZ. The periodic functions $u_{n, \vec{k}}(\vec{r})$ [and consequently $\psi_{n, \vec{k}}(\vec{r})$ ] should be understood as a twocomponent spinor(131). For a more thorough mathematical notation, we could write

$$
\left[u_{n, \vec{k}}\right](\vec{r})=\left[\begin{array}{c}
u_{n, \vec{k}, \uparrow}(\vec{r}) \\
u_{n, \vec{k}, \downarrow}(\vec{r})
\end{array}\right],
$$

in which $u_{n, \vec{k}, \uparrow}(\vec{r})$ and $u_{n, \vec{k}, \downarrow}(\vec{r})$ indicate the orbital function that is connected to the spin up and spin down projections, respectively. For simplicity, we do not consider the square brakets to denote the spinor character of the periodic functions $u_{n, \vec{k}}(\vec{k})$. Furthermore, the wave functions at points $\vec{k}$ and $-\vec{k}$ are connected by time-reversal symmetry(12), i. e., $\psi_{n,-\vec{k}}(\vec{r})=T \psi_{n, \vec{k}}(\vec{r})$, with $T=-i \sigma_{y} K$, in which $K$ is the complex conjugation operator. We usually refer to $\psi_{n,-\vec{k}}(\vec{r})$ as the Kramer's pair of the state $\psi_{n, \vec{k}}(\vec{r})$.

Rewriting the equation (2.1) and taking into account the Bloch's theorem, we have

$$
\mathbf{H} u_{n, \vec{k}}(\vec{r})=E_{n}(\vec{k}) u_{n, \vec{k}}(\vec{r})
$$

where the Hamiltonian $\mathbf{H}$ is given by

$$
\mathbf{H}=\underbrace{\frac{p^{2}}{2 m_{0}}+V(\vec{r})}_{\mathbf{H}_{\mathbf{0}}}+\underbrace{\frac{\hbar}{4 m_{0}^{2} c^{2}}[\vec{\nabla} V(\vec{r}) \times \vec{p}] \cdot \vec{\sigma}}_{\mathbf{H}_{\mathbf{S O}}}+\underbrace{\frac{\hbar^{2} k^{2}}{2 m_{0}}}_{\mathbf{H}_{\mathbf{k} 2}}+\underbrace{\frac{\hbar}{m_{0}} \vec{k} \cdot \vec{p}}_{\mathbf{H}_{\mathbf{k p}}}+\underbrace{\frac{\hbar^{2}}{4 m_{0}^{2} c^{2}}[\vec{\nabla} V(\vec{r}) \times \vec{k}] \cdot \vec{\sigma}}_{\mathbf{H}_{\mathbf{k S O}}},
$$

with the different terms indicated to further reference.

One possibility to deal with equation (2.3) is to expand the periodic functions around a point of the reciprocal space for all energy bands and calculate the different contributions of the Hamiltonian (2.4) using perturbation theory. All the materials investigated in this thesis have direct band gap at $\Gamma$ point $^{\dagger}$ (the FBZ center in Fig. 1.4), therefore, we proceed with the formulation assuming $\Gamma$ as the expansion point. Once we fix the expansion point, we have to decide how many energy bands (and periodic functions) should be included in the expansion. Including all the energy bands in the perturbative expansion would lead to a large Hamiltonian matrix. However, we can use Löwdin's perturbation technique(8) to reduce the size of the

\footnotetext{
$\dagger$ The carriers located around the vicinities of the band edges are responsible for the main optical and electrical features of the semiconductor.
} 
total matrix but still take into account the effect of all energy bands. In Löwdin's approach, the energy bands are divided in two different classes, namely A and B. Class A comprises the energy bands we want to describe and, consequently, the number of bands defines the size of the Hamiltonian matrix ${ }^{\ddagger}$. All the other energy bands of the system are included in class B. It is desirable that the energy bands chosen in class A are close in energy and interact strongly with each other but weakly with bands in class B. Also, we neglect the interaction of the energy bands in class B among themselves. Once we move away from the expansion point, the energy bands in class A and B can interact more strongly which means we are reaching the limits of the model. When this happens we can either modify the class A to include these important bands of class B or simply stop the calculation before this interacting region.

The expansion of the periodic functions into classes A and B is written as

$$
u_{n, \vec{k}}(\vec{r})=\sum_{\alpha}^{A} c_{n, \alpha}(\vec{k}) u_{\alpha, \Gamma}(\vec{r})+\sum_{\beta}^{B} c_{n, \beta}(\vec{k}) u_{\beta, \Gamma}(\vec{r}),
$$

with the assumption that the periodic functions $u_{n, \Gamma}$ are the solutions for the Hamiltonian term $\mathbf{H}_{\mathbf{0}}$ in equation (2.4), i. e., the unperturbed term ${ }^{\S}$. Therefore, the perturbed Hamiltonian $\mathbf{H}_{\mathbf{P}}$ comprises the remaining terms of equation (2.4), i. e.,

$$
\mathbf{H}_{\mathbf{P}}=\mathbf{H}_{\mathrm{SO}}+\mathbf{H}_{\mathbf{k} 2}+\mathbf{H}_{\mathrm{kp}}+\mathbf{H}_{\mathrm{kSO}} .
$$

Within Löwdin's approach, we can calculate several orders of perturbation for the different Hamiltonian terms in equation (2.6). To include the effects of the energy bands in class B, we must consider at least the second order contribution. In this thesis, similarly to the majority of papers in the literature, we consider terms up to second order of perturbation. Summarizing these perturbative terms, the first order matrix elements are given by

$$
H_{f}^{(1)}\left(\alpha, \alpha^{\prime}\right)=\left\langle\alpha\left|\mathbf{H}_{\mathbf{f}}\right| \alpha^{\prime}\right\rangle
$$

while the second order matrix elements are expressed as

\footnotetext{
$\ddagger$ If the number of energy bands inside class $\mathrm{A}$ is greater than one, we commonly refer to it as multiband approach

$\S$ It would be perfectly appropriate to consider the term $\mathbf{H}_{\mathbf{0}}+\mathbf{H}_{\mathrm{SO}}$ as unperturbed.
} 


$$
H_{f, g}^{(2)}\left(\alpha, \alpha^{\prime}\right)=\sum_{\beta}^{B} \frac{\left\langle\alpha\left|\mathbf{H}_{\mathbf{f}}\right| \beta\right\rangle\left\langle\beta\left|\mathbf{H}_{\mathbf{g}}\right| \alpha^{\prime}\right\rangle}{E_{\alpha \alpha^{\prime}}-E_{\beta}},
$$

with $\mathbf{H}_{\mathbf{f}}$ and $\mathbf{H}_{\mathbf{g}}$ being any of the terms identified in the Hamiltonian (2.6) and we expressed the periodic functions in Dirac notation. Explicitly, $\langle\vec{r} \mid \gamma\rangle=u_{\gamma, \Gamma}(\vec{r})$ with $\gamma=\left\{\alpha, \alpha^{\prime}\right\}$ in class A and $\gamma=\{\beta\}$ in class B.

How can we proceed with the formulation without knowing neither the effective potential nor the periodic functions? The solution for this conundrum is to use the gruppenpest (German for group plague), a term that refers to the complicated and abstract group theory arguments used in the early years of solid state physics.(2) In the next subsection we describe the role of group theory and its intimate relationship with the k.p method.

\subsubsection{The role of group theory}

Besides the translational symmetry properties of the crystal, the atoms in the unit cell can also be invariant under other symmetry operations, for instance, rotations, reflections, improper rotations, inversions, screw axis and glide planes.(13) These unit cell symmetry operations are identified as the factor group of the crystal.(12) For the particular crystal structures considered in this thesis, it is worth mentioning that $\mathrm{ZB}$ factor group is $T_{d}$ and symmorphic(10), while $\mathrm{WZ}$ factor group is isomorphic to $C_{6 v}$ and nonsymmorphic ${ }^{\top}$.(132)

The reciprocal space is also subjected to these symmetry constraints so that every $\vec{k}$ point belongs to a symmetry group, which is a subgroup of the crystal factor group. For instance, the $\Gamma$ point has the largest symmetry group, i. e., the point group isomorphic to the factor group of the crystal unit cell. Therefore, the $\Gamma$ point symmetry groups for $\mathrm{ZB}$ and $\mathrm{WZ}$ are $T_{d}$ and $C_{6 v}$, respectively. These symmetry groups of reciprocal space points dictate the symmetry operations that the wave functions are subjected as well as the degeneracy of the energy bands. In fact, the periodic functions are the basis states for the irreducible representations of the symmetry group.

Not only the periodic functions, but also the operators in the Hamiltonian terms that arise

\footnotetext{
$\bar{\pi}$ Symmorphic groups have only point group operations (rotations, reflections, improper rotations, inversions) while nonsymmorphic groups combine point group operations with translations by vectors that do not belong to the Bravais lattice of the crystal to create screw axis or glide plane operations.
} 
from (2.7) and (2.8) also belong to the $\vec{k}$ point symmetry group. Within the framework of group theory, it is possible to calculate the nonzero matrix elements used in equations (2.7) and (2.8) and build the functional form of Hamiltonian that, when diagonalized, provides the energy band dispersion. If the unperturbed term is $\mathbf{H}_{\mathbf{0}}$ then we refer to it as simple group approach. Considering $\mathbf{H}_{\mathbf{0}}+\mathbf{H}_{\mathbf{S O}}$ as the unperturbed term, then we call it double group approach. In this thesis, the simple group approach is usedll. Explicit calculations of these non-zero matrix elements based on the simple group formulation are nicely described in the references.(12, 134, 135)

Unfortunately, finding the nonzero elements is not the whole story for the k.p method. Although important physical insights can be extracted only from the functional form of the Hamiltonian, in order to achieve a more realistic and systematic investigation, real values should be addressed to these parameters. We discuss the approaches used in this thesis in the next subsection.

\subsubsection{Finding k.p parameters}

The most common approach to use the k.p method is to consider models and parameters already published in the literature. For III-V ZB and WZ nitrides semiconductors, a useful database for parameters (with more than 4500 citations) is the paper of Vurgaftman, Meyer and Ram-Mohan(100) from 2001. We used this approach for the GaAs and AlAs ZB parameters used in the spin laser paper(33) and for the InP ZB parameters used in the polytypic projects.(38, 39) But what if we want to consider new materials or new interactions in the Hamiltonians? Then we need to extract the k.p parameters from experiments or other theoretical techniques. In this thesis, two different possibilities were developed to obtain the parameters. We summarize them below:

(i) Effective parameters around the band edges: several experiments, for instance cyclotron resonance(136), or ab initio calculations provide information of the energy bands

\footnotetext{
| To the best of our knowledge, there is only one paper in the literature that deals with k.p Hamiltonians using the double group.(133)
} 
(effective masses and energy levels) in the vicinities of high-symmetry reciprocal space points. The main difficulty of using band edge information is to find analytical relations with the actual parameters of multiband Hamiltonians. Usually, these analytical connections are only possible in Hamiltonians with small number of bands and/or reduced number of interactions. The most successful case of analytical relations is the $6 \times 6$ Luttinger-Kohn Hamiltonian(9) for ZB materials, in which it is possible to extract the effective mass parameters $\left(\gamma_{1}, \gamma_{2}\right.$ and $\left.\gamma_{3}\right)$ based on the effective masses of the energy bands $\left(m_{H H}^{*}, m_{L H}^{*}\right.$ and $\left.m_{S O}^{*}\right)$. For WZ materials, however, the standard $6 \times 6$ valence band Hamiltonian with SOC(118) does not allow analytical solutions in all directions. We used the band edge data from De and Pryor(95) to obtain the InP WZ parameters employed in the polytypic papers. $(38,39)$ Since InP WZ from from De and Pryor(95) has small SOC splitting energies (compared to the crystal field energy) we started from the analytical relations between parameters and effective masses without SOC to find the values for $A_{2}$, $A_{4}$ and $A_{5}$ parameters, however, we had to fine tune the value of $A_{5}$ to correctly match the band structure. The value of $A_{6}$ was also fine tuned in a similar way. For $A_{1}$ and $A_{3}$ parameters, which appear only along $k_{z}$, the Hamiltonian including SOC allows analytical relations with the effective masses.

(ii) Numerical fitting: this would be the most versatile approach for novel multiband Hamiltonians, materials and physical interactions of the energy bands. However, it requires a calculated, or measured, band structure to be the target for the k.p Hamiltonian. Assuming this k.p Hamiltonian is suitable to describe the target band structure, it is possible to perform numerical fitting techniques to extract the parameters.(120-126) We used this approach, combined with $a b$ initio WIEN2k calculations(129), to find the parameters for an $8 \times 8$ k.p Hamiltonian including spin splitting properties for InAs and InP in WZ phase.(127) In Fig. 2.1, we describe the fitting scheme used in this project. The inputs required are the k.p model and the $a b$ initio band structure along different directions of the FBZ, especially directions that couple different $k_{x}, k_{y}$ and $k_{z}$ axes. We developed the numerical fitting code using Python language and the LMFIT package(137), which provides several minimization methods. For each minimization method and fixed $\vec{k}$ limit, we 
obtain one parameter set. Since we considered different minimization methods and varied the fitting limit, a large number of parameter sets is obtained. In order to find the best parameter set we calculate the residue between the fitted and $a b$ initio band structure and spin splittings. This best parameter set is then used as an input for the fitting approach in order to refine the obtained values.

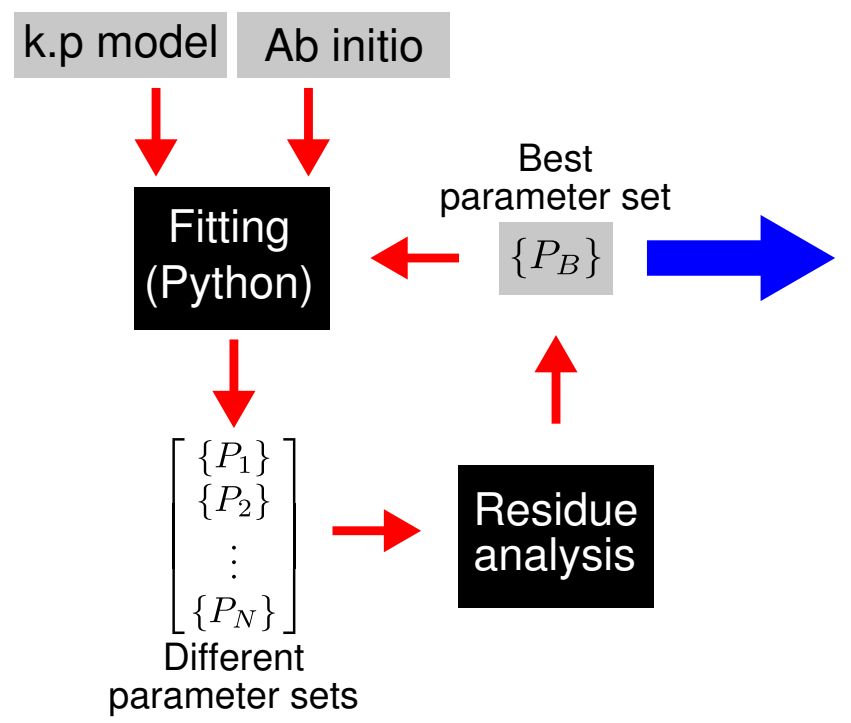

Figure 2.1 - Self-consistent fitting scheme to obtain the k.p parameters based on ab initio band structures, used in Ref.(127). The fitting approach provides a number of different parameter sets that are compared by residue analysis. The parameter set with a smaller residue is used as an input for the numerical fitting until the residue converges.

Source: By the author.

Up to this point, we have established the k.p formalism for bulk materials. In order to treat semiconductor systems with QC, such as quantum wells and superlattices, this bulk formalism should be revisited. In the next section, we address the theoretical treatment for quantum confined systems within the k.p formulation.

\subsection{Quantum confined systems}

In the previous section we showed the formulation of k.p method for bulk materials explicitly using the translational invariance property of the Bravais lattice, i. e., the Bloch's theorem. However, many semiconductor systems of interest are also subjected to the effect of external potentials, which lack this translational invariance. To illustrate such systems, we consider in 
Fig. 2.2 two possibilities of one-dimension external potentials, $\Phi(z)$. In Fig. 2.2(a), we show an external potential that can appear in a single material with an impurity center(9) or a $\delta$ doping region.(138) In Fig. 2.2(b), the external potential is the quantum well profile created by coupling different regions $\mathrm{A}$ and $\mathrm{B}$. These regions can represent, for instance, two different materials or the same material with two different crystal structures.
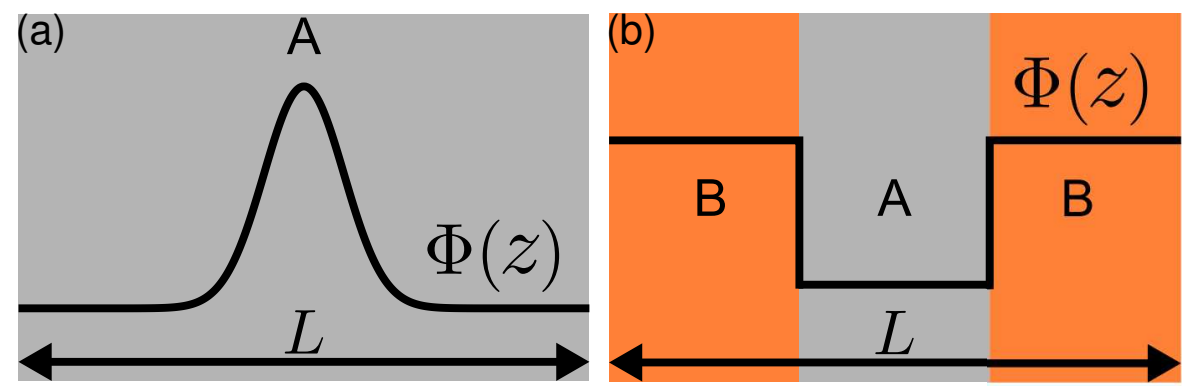

Figure 2.2 - One-dimension external potential (a) in a single material A and (b) for quantum well profile created by the regions B/A/B. Regions $\mathrm{A}$ and $\mathrm{B}$ can be two different materials or the same material with different crystal structures. The whole system has a dimension $L$.

Source: By the author.

In order to account for this external potential that is no longer periodic in the Bravais lattice, we must make some changes in the bulk formulation. Instead of using the Bloch's theorem, we replace the exponential part by a general function, $f(\vec{r})$, but still keep the periodic functions $u_{n, \Gamma}(\vec{r})$ to transfer the information of the bulk description into the new system, i. e., the specific parameters that define the bulk crystal. The Hamiltonian including a general three-dimensional external potential is given by

$$
\left\{\frac{p^{2}}{2 m_{0}}+V(\vec{r})+\frac{\hbar}{4 m_{0}^{2} c^{2}}[\vec{\nabla} V(\vec{r}) \times \vec{p}] \cdot \vec{\sigma}+\Phi(\vec{r})\right\} \psi(\vec{r})=E \psi(\vec{r}),
$$

and the total wave function can also be written using Löwdin's idea of distinct classes

$$
\psi_{n}(\vec{r})=\sum_{\alpha}^{A} f_{n, \alpha}(\vec{r}) u_{\alpha, \Gamma}(\vec{r})+\sum_{\beta}^{B} f_{n, \beta}(\vec{r}) u_{\beta, \Gamma}(\vec{r})
$$

with $f(\vec{r})$ being called the envelope function.

Starting with the wave function (2.10) and applying the Löwdin's formalism, the Hamiltonian matrix, with terms arising from (2.7) and (2.8), becomes a set of coupled differential equations for the functions $f_{n, \alpha}(\vec{r})$ and energies $E_{n}$. This approach, called the envelope function 
approximation (EFA), basically turns the wave vectors $\vec{k}$ of the bulk Hamiltonian into derivatives and the parameters into position-dependent functions.(14-19) We emphasize that it is necessary to consider different periodic functions $u(\vec{r})$ for different regions to obtain position-dependent parameters. Neglecting this assumption implies that the parameters of the Hamiltonian are constant numbers throughout all regions. Furthermore, considering different periodic functions and abrupt interfaces do not lead to the discontinuity of the total wave function, which is already discussed in several papers in the literature.(15-19)

Analytical solutions for this set of coupled differential equations can be found only in particular cases, for instance, with small number of bands and reduced coupling terms in the Hamiltonian.(139) The most general approach is to numerically solve the problem. In order to implement this numerical approach in the direct space, it is possible to apply finite difference or finite element methods.(140,141) An alternative approach, however, is to consider this set of coupled differential equations in the reciprocal space using the plane wave expansion (PWE), or Fourier transform, in the envelope functions, parameters and potentials. The Fourier expansion, besides being physical appealing, has two main advantages(17): first, the plane wave functions comprise an orthogonal and complete basis set and second, the first and second derivatives are also members of the basis set.

Since the Fourier transform automatically implies periodic boundary conditions, we can imagine that the whole system is a new unit cell for the periodic Fourier basis set. This Fourier unit cell is called super cell. Within this new picture, it is possible to assign a wave vector $\vec{k}$, that lies inside a super Brillouin zone ${ }^{* *}$, to the envelope functions, i. e., $f_{\alpha}(\vec{r}) \rightarrow f_{\alpha, \vec{k}}(\vec{r})$. Writing the envelope functions in the PWE form we have

$$
\begin{aligned}
f_{\alpha, \vec{k}}(\vec{r}) & =e^{i \vec{k} \cdot \vec{r}} g_{\alpha}(\vec{r}) \\
& =e^{i \vec{k} \cdot \vec{r}} \sum_{\vec{K}} e^{i \vec{K} \cdot \vec{r}} g_{\alpha, \vec{K}}(\vec{k}) \\
& =\sum_{\vec{K}} e^{i(\vec{k}+\vec{K}) \cdot \vec{r}} g_{\alpha, \vec{K}}(\vec{k}),
\end{aligned}
$$

\footnotetext{
** The super Brillouin zone along the confinement directions has reduced dimensions compared to the FBZ of the bulk crystal. The adjective super is related to the definition of the super cell in direct space, which is typically larger than the bulk unit cell.
} 
with the parameters in the Hamiltonian and external potentials also Fourier expanded in the form $^{\dagger \dagger}$

$$
\chi(\vec{r})=\sum_{\Delta \vec{K}} e^{i \Delta \vec{K} \cdot \vec{r}} \chi_{\Delta \vec{K}}
$$

with

$$
\Delta \vec{K}=\vec{K}-\vec{K}^{\prime}
$$

Within the PWE formalism applied to the EFA equations ${ }^{\ddagger \ddagger}$, we obtain the symmetrization properties of the wave vectors

$$
\begin{aligned}
\vec{k} & \rightarrow \frac{1}{2}\left[(\vec{k}+\vec{K})+\left(\vec{k}+\vec{K}^{\prime}\right)\right] \\
k_{l} k_{m} & \rightarrow \frac{1}{2}\left[\left(k_{l}+K_{l}\right)\left(k_{m}+K_{m}^{\prime}\right)+\left(k_{m}+K_{m}\right)\left(k_{l}+K_{l}^{\prime}\right)\right],
\end{aligned}
$$

with

$$
\left\{K_{j}, K_{j}^{\prime}\right\}=j \frac{2 \pi}{L_{j}} \quad, \quad \frac{\pi}{L_{j}} \leq k_{j} \leq \frac{\pi}{L_{j}} \quad, \quad j=x, y, z .
$$

with $j=0, \pm 1, \pm 2, \cdots$. It is important to emphasize that these symmetrization rules follow directly from the Hermitian properties of the EFA equations.

Including the symmetrization properties of Fourier wave vectors $\vec{K}$ and Fourier coefficients of $\Delta \vec{K}$ for parameters and external potentials, the Hamiltonian in the PWE and EFA approach becomes a numerical matrix. For a nonzero number $N$ of Fourier vectors $\vec{K}$, we consider $2 N+1$ Fourier coefficients for each envelope function and $4 N+1$ Fourier coefficients for parameters and external potentials. The size of the resulting matrix is therefore $[M(2 N+1)]^{2}$ with $M$ being the size of the bulk Hamiltonian used. Diagonalizing this matrix numerically, we obtain the energy values, $E_{n}(\vec{k})$, and the Fourier coefficients, $g_{n, \alpha, \vec{K}}(\vec{k})$, associated to the envelope functions.

Qualitatively, we can summarize in Fig. 2.3 the influence of an external potential to the bulk energy bands. Regardless of the shape or physical origin of the potential, we usually refer to this influence by QC effect, since this new potential creates additional quantum confined states.

$\dagger \dagger$ The summation over $\vec{K}$ and $\Delta \vec{K}$ is not arbitrary. It is the result of applying the PWE formalism to the EFA set of equations, described in detail in section 5.2 of Ref.(134) and also in section 3.3 of Ref.(142).

$\ddagger \ddagger$ For more details and examples of the combined EFA and PWE approaches in k.p Hamiltonians described here, please look at Refs. $(38,39,134,138,142)$. 
Let us consider two bulk bands, depicted in Fig. 2.3(a), that are separated by an energy gap. The wave vector vector $k_{c}$ is along the confinement direction and $\pi / a$ is the bulk FBZ edge. Increasing the size of the system to $L$ (the length of the super cell), automatically reduces the FBZ edge to $\pi / L$ (that defines the super Brillouin zone edge). This effect alone in the band structure is the zone folding, or the empty lattice approximation(143), shown in Fig. 2.3(b). In other words, in Fig. 2.3(b) we turned off the interaction with the confinement potential and show the energy bands in a reduced zone scheme(1). Turning on the interaction with the external potential, the QC effect appears in the energy bands, illustrated in Fig. 2.3(c). The effect of QC is two-fold: it creates addition energy gaps and shifts the energy values away from the bulk energy bands. The resulting energy values due to the quantum confined effect are called minibands ${ }^{\S \S}$.(130) If this external potential is responsible for a strong QC, the minibands dispersion are reduced to flat lines and shifted further away from the bulk energy bands, depicted in Fig.2.3(d). For the reciprocal space directions perpendicular to the growth direction, $k_{p}$, the energy bands are called subbands and may look similar to what is shown in 2.3(e). Although some subbands can resemble the bulk energy bands, QC effects can drastically change the dispersion along $k_{p}$ direction.

Within this picture described in Fig. 2.3, what is the difference between a quantum well and a superlattice? The answer to this question lies in the minibands. For a quantum well, we are interested in the confined states, which have flat miniband dispersion. The wave vector $k_{c}$ in this case carries no physics. On the other hand, for a superlattice there is indeed a repetition of the super cell, i. e., the physical system is a periodic sequence of quantum wells, and the wave vector $k_{c}$ has physical meaning and relevance. Although the theoretical formalism of the EFA and PWE is general, it is convenient to check the physical meaning of the wave vector $k_{c}$ in the particular system under investigation. In other words, $k_{c}$ can be just a mathematical feature or a real physical quantity and, therefore, a careful look at the physical system should provide the answer.

Now that we have established the theoretical formalism to calculate the electronic structure

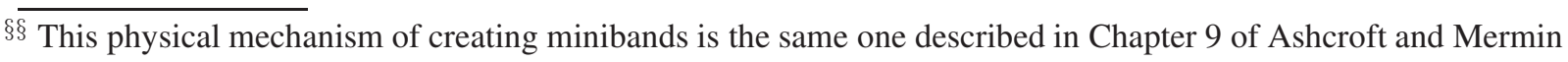
book(1), showing the appearance of energy bands in a crystal with periodic potential. The super Brillouin zone edges mentioned in this thesis are the Bragg planes discussed in the book 
of bulk materials and quantum confined systems using the k.p method, we may investigate interesting observable properties. In the next chapter we discuss the light-matter interaction in semiconductor systems, by investigating the absorption and gain coefficients.
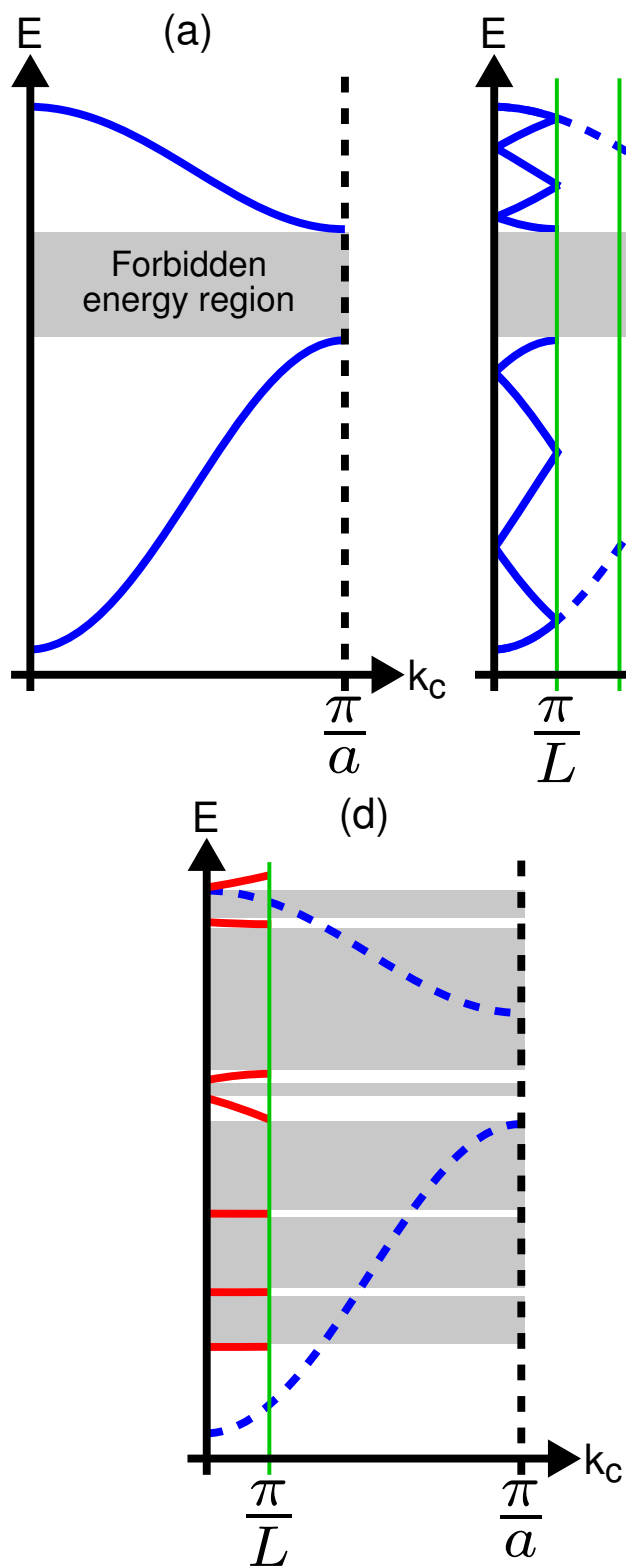

(b)

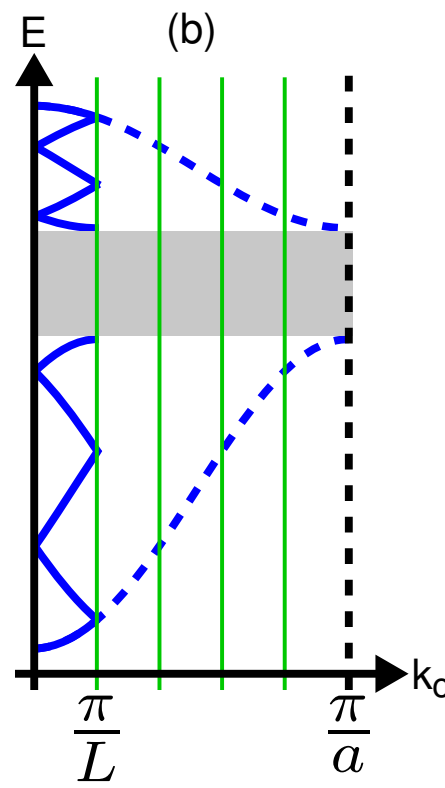

(e)

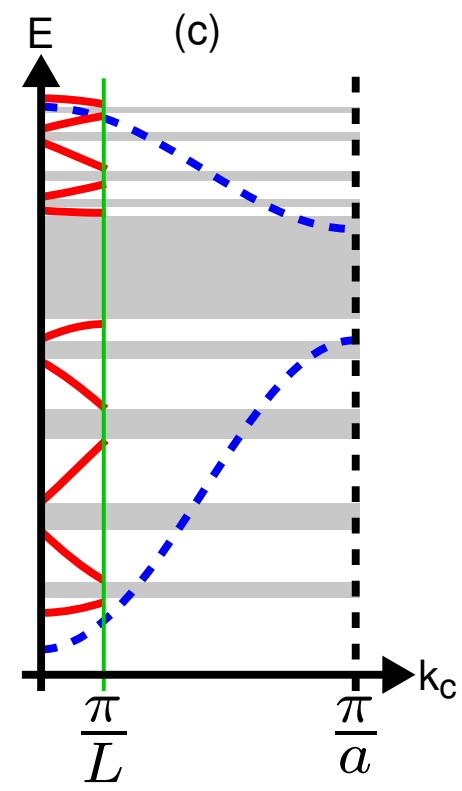

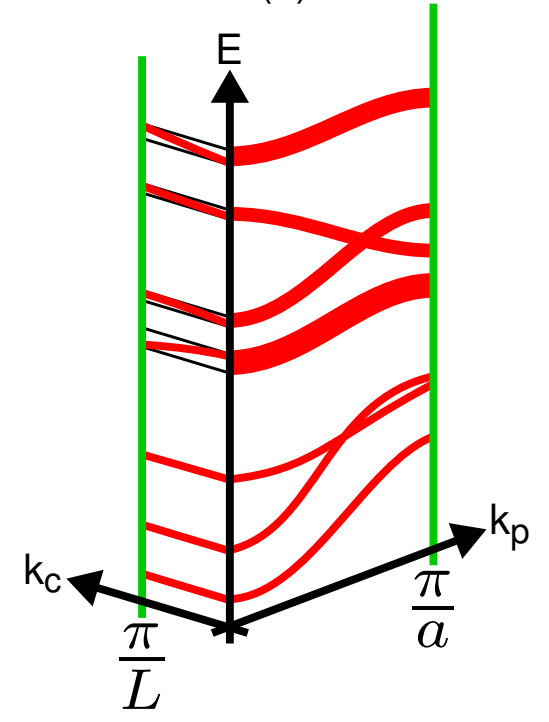

Figure 2.3 - A qualitatively description of the QC effect in energy bands. (a) Bulk energy bands. (b) Zone folding. (c) Energy shift and mini gaps induced in the energy bands. (d) Minibands in strong QC. (e) Minibands and subbands.

Source: By the author. 


\section{Absorption and gain coefficients}

The interaction between an electromagnetic wave and the semiconductor system is a quantum mechanical process, often called light-matter or electron-photon interaction(44, 66, 144, 145), very useful to probe the electronic structure of solid materials. The electronic energy levels and their wave functions define the optical selection rules, i. e., the energy and polarization of photons allowed during an optical transition (either emission or absorption). In a laser, the light-matter interaction happens in the active region when electrons and holes recombine and emit light or, when the electron-hole pair is created and absorbs light. The condition for photons to be emitted or absorbed in the active region is given by the gain coefficient, defined as the number of photons emitted per second per unit volume over the number of injected photons per second per unit area. From this definition, negative values indicate the lack of emitted photons, therefore, absorption. In this chapter, we address the theoretical formulation of the absorption and gain coefficient and develop the spin-dependent gain model for spin lasers. To show the connection between these optical (absorption, gain and spin-dependent gain) coefficients, we apply this formalism to a fully analytical two-band model.

\subsection{General formulation}

The Hamiltonian for light-matter interaction can be obtained by using the minimal coupling $(6,66,110)$ in the crystal Hamiltonian given in equation $(2.1)$, i. e.,

$$
\vec{p} \rightarrow \vec{p}+e \vec{A}
$$

in which $e$ is the modulus of the electron charge and $\vec{A}$ is the vector potential.

In order to obtain the gain coefficient formulation, an intrinsic material property, it is possible to use the Fermi's golden rule within the time-dependent perturbation theory(66) or a second 
quantization formalism within a free-carrier approach.(144, 145) Regardless of the approach used, the gain coefficient for light polarization, $a$, is given by $(33,66,145)$

$$
g^{a}(\hbar \omega)=\left(\frac{4 \pi^{2} e^{2}}{n_{r} c_{l} \varepsilon_{0} \omega \Omega m_{0}^{2}}\right) \sum_{c, v, \vec{k}}\left|p_{c, v, \vec{k}}^{a}\right|^{2}\left(f_{c, \vec{k}}-f_{v, \vec{k}}\right) \delta\left(\hbar \omega_{c, v, \vec{k}}-\hbar \omega\right),
$$

where the summation indices $c$ and $v$ label the conduction and valence subbands, respectively, $\vec{k}$ is the wave vector, $p_{c, v, \vec{k}}^{a}$ is the interband dipole transition amplitude, $f_{c(v), \vec{k}}$ is the Fermi-Dirac distribution for the electron occupancy in the conduction (valence) subbands, $\hbar$ is Planck's constant, $\omega_{c, v, \vec{k}}$ is the interband transition frequency, and $\delta$ is the Dirac delta function, which is often replaced to include broadening effects for finite lifetimes. $(66,144)$ For the constants outside the summation, $e$ is the electron charge (the modulus, $>0$ ), $n_{r}$ is the dominant real part of the refractive index of the material(145), $c_{l}$ is the speed of light (subindex $l$ added to avoid confusion with the conduction subband index $c$ ), $\varepsilon_{0}$ is the vacuum permittivity, $\Omega$ is the quantum well volume* and $m_{0}$ is the free-electron mass.

The Fermi-Dirac distribution is given by

$$
f_{x, \vec{k}}=\frac{1}{1+\exp \left\{\beta\left[E_{x}(\vec{k})-\mu_{X}\right]\right\}},
$$

with $x=(v, c), X=(V, C), \mu$ is the chemical potential, $\beta=1 / k_{b} T$ and $k_{b}$ is the Boltzmann constant. The capital subindices for the chemical potential indicate that the occupation takes into account all the conduction and valence subbands.

Adding carriers to the laser active region, either by electrical or optical injection, creates a quasi-equilibrium state defined by the chemical potentials. In other words, the pumping mechanism creates free electrons in conduction band and free holes in the valence band that can recombine to emit light. The specific conditions that this pumping mechanism has to fulfill in order to achieve the lasing operation are discussed within the simple analytical model of section 3.3. Let us first understand the mathematical description of injecting carriers in the active region. Given an injected carrier density $N$, its relation to the chemical potential is expressed

\footnotetext{
* It is convenient to write the quantum well volume as $\Omega=L A$, in which $L$ is the width and $A$ is the area of the quantum well.
} 
by the equation

$$
N=\int_{0}^{\infty} \mathrm{d} E \frac{\rho(E)}{1+e^{\beta(E-\mu)}},
$$

with the occupation of the energy bands being determined by the density of states, or DOS, of the system, defined as(130)

$$
\rho(E)=\frac{1}{D} \sum_{\vec{k}} \delta\left[E_{\vec{k}}-E\right],
$$

where $D$ is the dimension of the system (volume, area or length). Therefore, the DOS has units of $\mathrm{eV}^{-1}[D]^{-1}$ and the integral in energy leads to a carrier density in units of $[D]^{-1}$.

At $T=0 \mathrm{~K}$, the chemical potential is called the Fermi energy, i. e., the energy of the last occupied state, and the equation (3.4) is simplified to

$$
N=\int_{0}^{E_{f}} \mathrm{~d} E \rho(E)
$$

In Fig. 3.1(a) we show the quasi-equilibrium situation under carrier injection defined by the chemical potential $\mu_{C(V)}$ for the conduction (valence) band. Since conduction and valence bands have different curvatures, the occupation can be quite different for the same carrier density of electrons and holes. In the gray regions, the carriers have no spin polarization, i. e., there is an equal amount of spin up and spin down carriers (indicated by the red and blue arrows). The case with spin polarized carriers is shown in Fig. 3.1(b) and is addressed in the next section.
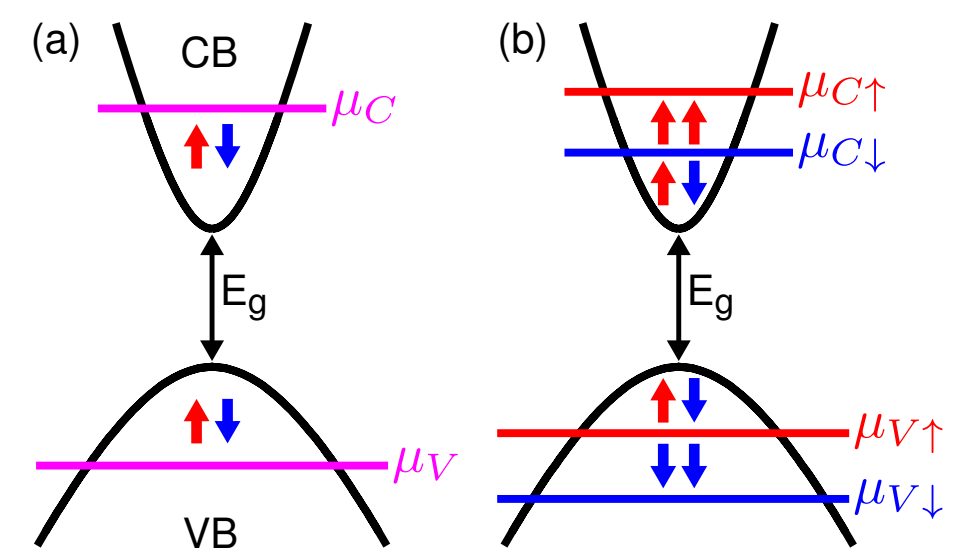

Figure 3.1 - Quasi-equilibrium configuration in the band structure due to carrier injection (a) without spin polarization and (b) assuming spin-polarized carriers. The red arrows indicate spin up electrons and the blue arrows indicate spin down electrons.

Source: By the author. 


\subsection{Spin-polarized gain}

In order to investigate the spin laser active region $(32,33)$, we allow the injected carriers to be spin polarized, i. e., there is a net spin imbalance in the system. This spin imbalance in the active region is quantified by the spin polarization

$$
P_{C(V)}=\frac{N_{C(V) \uparrow}-N_{C(V) \downarrow}}{N_{C(V)}},
$$

with $C(V)$ indicating the conduction (valence) band and the total carrier density written in terms of the spin up and spin down ${ }^{\dagger}$ carrier densities

$$
N_{C(V)}=N_{C(V) \uparrow}+N_{C(V) \downarrow}
$$

Given the total carrier density $N_{C(V)}$ and the spin polarization $P_{C(V)}$, we can find $N_{C(V) \uparrow}$ and $N_{C(V) \downarrow}:$

$$
\left\{\begin{array}{l}
N_{C(V) \uparrow}=\frac{N_{C(V)}}{2}\left[1+P_{C(V)}\right] \\
N_{C(V) \downarrow}=\frac{N_{C(V)}}{2}\left[1-P_{C(V)}\right]
\end{array} .\right.
$$

Since assuming spin polarization is more general, the spin unpolarized case is a particular situation with

$$
N_{C(V) \uparrow}=N_{C(V) \downarrow}=\frac{N_{C(V)}}{2},
$$

and, consequently, the chemical potentials for spin up and spin down carriers are the same

$$
\mu_{C(V)}=\mu_{C(V) \uparrow}=\mu_{C(V) \downarrow}
$$

The quasi-equilibrium situation in the spin polarized case is shown in Fig. 3.1(b). For the conduction band we depict a situation with $P_{C}>0$, i. e., there is a majority of spin up electrons. Energies below $\mu_{C \downarrow}$ have both spin up and spin down electrons, while energies ranging from $\mu_{C \downarrow}$ to $\mu_{C \uparrow}$ contain only spin up electrons. In the valence band we show the opposite case with $P_{V}<0$, so that energies above $\mu_{V \uparrow}$ allow both spin up and spin down holes while energy values

\footnotetext{
$\uparrow^{\dagger}$ For clarity, spin up $(\uparrow)$ and spin down $(\downarrow)$ the projections of spin $1 / 2$ angular momentum, i. e., eigenvalues of the $\sigma_{z}$ Pauli matrix.
} 
ranging from $\mu_{V \uparrow}$ to $\mu_{V \downarrow}$ allow only spin down holes. Our convention for valence band states follow the usual definition of rate equations $(65,70,71)$, i. e., we consider the electron picture and the empty states in the valence band indicate possible states that electrons can occupy after recombination. The spin polarization $P_{V}$ also refers to these empty states.

In order to calculate the spin resolved chemical potentials, it is helpful to write the eigenvectors in the form

$$
\left|\psi_{\vec{k}}\right\rangle=\left|\psi_{\vec{k}, \uparrow}\right\rangle \otimes|\uparrow\rangle \oplus\left|\psi_{\vec{k}, \downarrow}\right\rangle \otimes|\downarrow\rangle
$$

with $\left|\psi_{\vec{k}, \uparrow(\downarrow)}\right\rangle$ representing the orbital contribution for spin up (down) projections. This is the two-component spinor description [equation (2.2)] written in Dirac notation.

We can write the spin resolved DOS

$$
\rho_{\uparrow(\downarrow)}(E)=\frac{1}{D} \sum_{\vec{k}}\left|\left\langle\uparrow(\downarrow) \mid \psi_{\vec{k}}\right\rangle\right|^{2} \delta\left[E_{\vec{k}}-E\right],
$$

that recovers the total DOS (3.13) if we add both spin contributions

$$
\rho(E)=\rho_{\uparrow}(E)+\rho_{\downarrow}(E),
$$

since $\left|\left\langle\uparrow \mid \psi_{\vec{k}}\right\rangle\right|^{2}+\left|\left\langle\downarrow \mid \psi_{\vec{k}}\right\rangle\right|^{2}=1$.

The spin-dependent chemical potentials can be calculated in the same approach as equation (3.4) for the unpolarized case:

$$
N_{\uparrow(\downarrow)}=\int_{0}^{\infty} \mathrm{d} E \frac{\rho_{\uparrow(\downarrow)}(E)}{1+e^{\beta\left[E-\mu_{\uparrow(\downarrow)}\right]}} .
$$

Finally, since the dipole transition element does not flip spins ${ }^{\ddagger}$, we can write the total gain (3.2) as the contribution of spin up and spin down transitions

$$
g^{a}(\hbar \omega)=g_{\uparrow}^{a}(\hbar \omega)+g_{\downarrow}^{a}(\hbar \omega) .
$$

Before we move on to the next section, it is important to mention the timescales involved in the gain model: (i) $\tau_{i}$, the intraband scattering time; (ii) $\tau_{r}$, the radiative recombination

\footnotetext{
$\ddagger$ This transition matrix element is mediated by the operator $\hat{a} \cdot \vec{P}$, with $\hat{a}$ being the light polarization and $\vec{P}$ being the linear momentum, that does not act in the spin $1 / 2$ Hilbert space.
} 
time of electrons and holes; and (iii) $\tau_{s}$, the spin relaxation time (in this case, let us focus on electrons). Typically for ZB [001] GaAs-based quantum wells at room temperature, $\tau_{i} \sim$ $10^{-13} \mathrm{~s}(144), \tau_{r} \sim 10^{-10} \mathrm{~s}(146,147)$ for spontaneous radiative recombination and $\tau_{s} \sim 10^{-11} \mathrm{~s}$ $\S(150)$. For the conventional laser, without spin polarization, only $\tau_{i}$ and $\tau_{r}$ matter. Once the carriers are injected in the laser active region, the time that carriers take to decay to lower energies is given by $\tau_{i}$, and, since $\tau_{i}$ is smaller than $\tau_{r}$, the quasi-equilibrium situation of Fig. 3.1(a) is created(66). In other words, before carriers recombine they occupy the lowest energy states and this occupation can be described by the chemical potentials of Fig. 3.1(a). For spin lasers, in which electrons have a net spin imbalance, $\tau_{s}$ should also be taken into account. At first glance, it seems that we should have $\tau_{s}>\tau_{r}>\tau_{i}$ in order to achieve spin laser operation and this is not the case for the values mentioned above. The key factor to notice here is that carrier recombination time, $\tau_{r}$, under stimulated emission is reduced and, given the values mentioned above, the condition $\tau_{s}>\tau_{r}>\tau_{i}$ is satisfied. Furthermore, rate equations show that even at the condition $\tau_{s}=\tau_{r}$, nonzero spin polarization of electrons is allowed in the active region(70) and spin-dependent recombination takes place. In the next section, we show an analytical example to address the specific microscopic conditions to achieve the lasing regime considering carriers with and without spin polarization.

\subsection{Simple analytical model}

To obtain some physical insight about the absorption and gain coefficients of more complex systems, let us investigate a simple two-band model with crystal wave vector dispersion in the $k_{x} k_{y}$ plane. The two-band refers to one conduction and one valence band, however, each of these bands is two-fold degenerate in spin. Although this may seem a simplified toy model, it is in fact reasonable. Physically, we can think that the valence band is comprised by only one kind of hole ( $\mathrm{HH}$ in $\mathrm{ZB}$ for example), therefore this assumption holds. Furthermore, in rate equations $(65,70,71)$ the recombination of spin up (down) electrons with spin up (down) holes

\footnotetext{
$\S$ The spin relaxation mechanism dominant in ZB semiconductor systems is called Dyakonov-Perel.(135, 148, 149) The BIA creates a effective magnetic field for electrons with direction that depends on the carrier momentum. As a consequence, a spin precession happens between collisions with the impurities, leading to a net electronic spin relaxation after many scatterings.
} 
is assumed to provide only one type of light polarization, therefore, it is also helpful to imagine that this two-band model is the microscopic version of the simplified rate equation approach.

The energy dispersion for conduction band is given by

$$
E_{c}(\vec{k})=E_{g}+\left(\frac{\hbar^{2}}{2 m_{0}}\right) \frac{1}{m_{c}^{*}}\left(k_{x}^{2}+k_{y}^{2}\right),
$$

with the following two-fold degenerate eigenstates

$$
\begin{aligned}
& |c \uparrow, \vec{k}\rangle=\left|\lambda_{\uparrow, \vec{k}}\right\rangle \otimes|\uparrow\rangle \\
& |c \downarrow, \vec{k}\rangle=\left|\lambda_{\downarrow, \vec{k}}\right\rangle \otimes|\downarrow\rangle .
\end{aligned}
$$

On the other hand, the energy dispersion for valence band is given by

$$
E_{v}(\vec{k})=-\left(\frac{\hbar^{2}}{2 m_{0}}\right) \frac{1}{m_{v}^{*}}\left(k_{x}^{2}+k_{y}^{2}\right),
$$

with the following two-fold degenerate eigenstates

$$
\begin{aligned}
& |v \uparrow, \vec{k}\rangle=\left|\theta_{\uparrow, \vec{k}}\right\rangle \otimes|\uparrow\rangle \\
& |v \downarrow, \vec{k}\rangle=\left|\theta_{\downarrow, \vec{k}}\right\rangle \otimes|\downarrow\rangle .
\end{aligned}
$$

Let us assume the dipole selection rules for $S^{+}$and $S^{-}$light polarizations to be

$$
\begin{aligned}
& \left|p_{c \uparrow, v \uparrow, \vec{k}}^{+}\right|^{2}=\left|p_{c \downarrow, v \downarrow, \vec{k}}^{-}\right|^{2} \neq 0 \\
& \left|p_{c \uparrow, v \uparrow, \vec{k}}^{-}\right|^{2}=\left|p_{c \downarrow, v \downarrow, \vec{k}}^{+}\right|^{2}=0,
\end{aligned}
$$

with the possibility that the coupling between states with same spin leads to only one kind of light polarization.

Taking into account the summation over energy bands from equation (3.2), the gain coefficients for $S^{+}$and $S^{-}$light polarizations are given by

$$
\begin{aligned}
& g^{+}(\hbar \omega)=\left(\frac{4 \pi^{2} e^{2}}{n_{r} c_{l} \varepsilon_{0} \omega \Omega m_{0}^{2}}\right) \sum_{\vec{k}}\left|p_{c \uparrow, v \uparrow, \vec{k}}^{+}\right|^{2}\left(f_{c \uparrow, \vec{k}}-f_{v \uparrow, \vec{k}}\right) \delta\left(\hbar \omega_{c, v, \vec{k}}-\hbar \omega\right) \\
& g^{-}(\hbar \omega)=\left(\frac{4 \pi^{2} e^{2}}{n_{r} c_{l} \varepsilon_{0} \omega \Omega m_{0}^{2}}\right) \sum_{\vec{k}}\left|p_{c \downarrow, v \downarrow, \vec{k}}^{-}\right|^{2}\left(f_{c \downarrow, \vec{k}}-f_{v \downarrow, \vec{k}}\right) \delta\left(\hbar \omega_{c, v, \vec{k}}-\hbar \omega\right),
\end{aligned}
$$


with the Fermi-Dirac distribution for the valence band described in the electron picture, i. e., it quantifies the states occupied by electrons [as shown in Fig. 3.2(c)].

The allowed photon energies are obtained by the difference of conduction and valence bands

$$
\begin{aligned}
\hbar \omega_{c, v, \vec{k}} & =E_{c}(\vec{k})-E_{v}(\vec{k}) \\
& =E_{g}+\left(\frac{\hbar^{2}}{2 m_{0}}\right)\left(\frac{1}{m_{c}^{*}}+\frac{1}{m_{v}^{*}}\right)\left(k_{x}^{2}+k_{y}^{2}\right) \\
& =E_{g}+\left(\frac{\hbar^{2}}{2 m_{0}}\right) \frac{1}{m_{r}^{*}}\left(k_{x}^{2}+k_{y}^{2}\right),
\end{aligned}
$$

where we used the definition of reduced mass to simplify the notation

$$
\frac{1}{m_{r}^{*}}=\frac{1}{m_{c}^{*}}+\frac{1}{m_{v}^{*}}
$$

We can remove the wave vector dependence by defining the reduced energy(144)

$$
\varepsilon=\left(\frac{\hbar^{2}}{2 m_{0}}\right) \frac{1}{m_{r}^{*}}\left(k_{x}^{2}+k_{y}^{2}\right),
$$

which gives

$$
E_{c}(\varepsilon)=E_{g}+\frac{m_{r}^{*}}{m_{c}^{*}} \varepsilon
$$

and

$$
E_{v}(\varepsilon)=-\frac{m_{r}^{*}}{m_{v}^{*}} \varepsilon
$$

In order to model the nonzero dipole transition elements as function of the reduced energy, let us assume the dispersion

$$
\left|p_{c \uparrow, v \uparrow, \vec{k}}^{+}\right|^{2}=\left|p_{c \downarrow, v \downarrow, \vec{k}}^{-}\right|^{2}=\left(\frac{m_{0}}{\hbar}\right)^{2} \frac{P^{2}}{1+\gamma \varepsilon}
$$

with $\gamma \geq 0$ having dimension of $\mathrm{eV}^{-1}$ and $P$ having dimension of $\mathrm{eV} \AA$. The parameter $\gamma$ is added to model the decay of the dipole transition amplitude as we move away from the band edge. In a more realistic system, this dipole transition matrix element not only decays but also changes the light polarization contribution, i. e., as $\left|p_{c \uparrow, v \uparrow, \vec{k}}^{+}\right|^{2}$ decreases, $\left|p_{c \uparrow, v \uparrow, \vec{k}}^{-}\right|^{2}$ can increase.

\footnotetext{
ฯ The effective masses $m_{c}^{*}, m_{v}^{*}$, and $m_{r}^{*}$, are in units of $m_{0}$, i. e., they are just numbers since $m_{0}$ is already in the
} equation. 
The same happens for $\left|p_{c \downarrow, v \downarrow, \vec{k}}^{-}\right|^{2}$ and $\left|p_{c \downarrow, v \downarrow, \vec{k}}^{+}\right|^{2}$.

For the Fermi-Dirac distribution, we have

$$
\begin{aligned}
F_{\uparrow(\downarrow)}(\varepsilon) & =f_{c \uparrow(\downarrow), \vec{k}}-f_{v \uparrow(\downarrow), \vec{k}} \\
& =\frac{1}{1+\exp \left\{\beta\left[E_{c}(\vec{k})-\mu_{C \uparrow(\downarrow)}\right]\right\}}-\frac{1}{1+\exp \left\{\beta\left[E_{v}(\vec{k})-\mu_{V \uparrow(\downarrow)}\right]\right\}} \\
& =\frac{1}{1+\exp \left[\beta\left(E_{g}+\frac{m_{r}^{*}}{m_{c}^{*}} \varepsilon-\mu_{C \uparrow(\downarrow)}\right)\right]}-\frac{1}{1+\exp \left[\beta\left(-\frac{m_{r}^{*}}{m_{v}^{*}} \varepsilon-\mu_{V \uparrow(\downarrow)}\right)\right]}
\end{aligned}
$$

The summation in $\vec{k}$ of the Dirac delta function has the same form of the DOS defined in equation (3.13), however, in this case, it combines two different energy bands. This combined DOS is labeled joint density of states, or simply JDOS, and given by

$$
J_{\uparrow(\downarrow)}(\hbar \omega)=\frac{1}{A} \sum_{\vec{k}} \delta\left(\hbar \omega_{c, v, \vec{k}}-\hbar \omega\right),
$$

which for this $2 \mathrm{D}$ dispersion we are investigating is simply

$$
J_{\uparrow(\downarrow)}(\varepsilon)=\left(\frac{m_{0} m_{r}^{*}}{2 \pi \hbar^{2}}\right) \Theta(\varepsilon),
$$

with

$$
\Theta(\varepsilon)=\left\{\begin{array}{ll}
1, & \varepsilon \geq 0 \\
0, & \varepsilon<0
\end{array},\right.
$$

where the reduced energy can also be related to the photon energy by the expression

$$
\varepsilon=\hbar \omega-E_{g}
$$

The final expression of the gain coefficient for $S^{+}$and $S^{-}$light polarizations is therefore

$$
\begin{aligned}
& g^{+}(\varepsilon)=C_{0} \frac{F_{\uparrow}(\varepsilon)}{(1+\gamma \varepsilon)\left(\varepsilon+E_{g}\right)} \Theta(\varepsilon) \\
& g^{-}(\varepsilon)=C_{0} \frac{F_{\downarrow}(\varepsilon)}{(1+\gamma \varepsilon)\left(\varepsilon+E_{g}\right)} \Theta(\varepsilon),
\end{aligned}
$$

with

$$
C_{0}=\frac{2 \pi m_{0} m_{r}^{*} e^{2} P^{2}}{n_{r} c_{l} \varepsilon_{0} L \hbar^{3}} .
$$


For this particular case ${ }^{\|}$, it is possible to find an analytical solution for the chemical potential as a function of carrier density, which is

$$
\begin{aligned}
& \mu_{C \uparrow(\downarrow)}=E_{g}-\frac{1}{\beta} \ln \left[\frac{1}{\exp \left(\frac{2 \beta \pi \hbar^{2}}{m_{0} m_{c}^{*}} N_{C \uparrow(\downarrow)}\right)-1}\right] \\
& \mu_{V \uparrow(\downarrow)}=\frac{1}{\beta} \ln \left[\frac{1}{\exp \left(\frac{2 \beta \pi \hbar^{2}}{m_{0} m_{v}^{*}} N_{V \uparrow(\downarrow)}\right)-1}\right] .
\end{aligned}
$$

In Figs. 3.2(a)-(c), we present the band structure, the DOS and the occupancy at $T=300 \mathrm{~K}$, respectively, for the two-band model described in this section. Since we are considering a simple parabolic model, the properties are ruled by the values of the effective masses. This is reflected in the different concavities of the energy bands and different multiplication constants in the DOS. Due to the two-fold spin degeneracy, the DOS for spin up and spin down electrons are the same. For the occupancy, the larger effective mass in the valence band provides a value of chemical potential outside the energy band. At $T=0 \mathrm{~K}$, however, the Fermi energy is negative, i. e., it lies inside the valence band.

For the optical properties related to the two-band model, we present the gain coefficient at $T=0 \mathrm{~K}$ in Fig. 3.3(a) without carriers in the system. This is basically the negative value of the absorption coefficient. Such analysis is very interesting to understand the band-edge transitions and how the carriers are absorbed under different light polarization conditions. Also in Fig. 3.3(a) we show the effect of the parameter $\gamma$, which essentially decreases the strength of the optical transitions in the system.

In Fig. 3.3(b) we show the gain coefficient at $T=300 \mathrm{~K}$ varying the carrier density injected in the system and assuming $\gamma=0.1 \mathrm{eV}^{-1}$. Let us start with the case without spin polarized carriers, solid lines in Fig. 3.3(b), to understand how the lasing regime is connected to the band structure of the system, which describes the active region. The pumping mechanism of the laser essentially adds carriers to the active region, and the amount of carrier density added to the system indicates the gain or absorption regime. The criteria to achieve positive gain is that the

$\|$ This is the case of the free noninteracting electron gas in two dimensions where the Sommerfeld expansion fails. For instance, see problem 2.1 from Ashcroft and Mermin book.(1) 


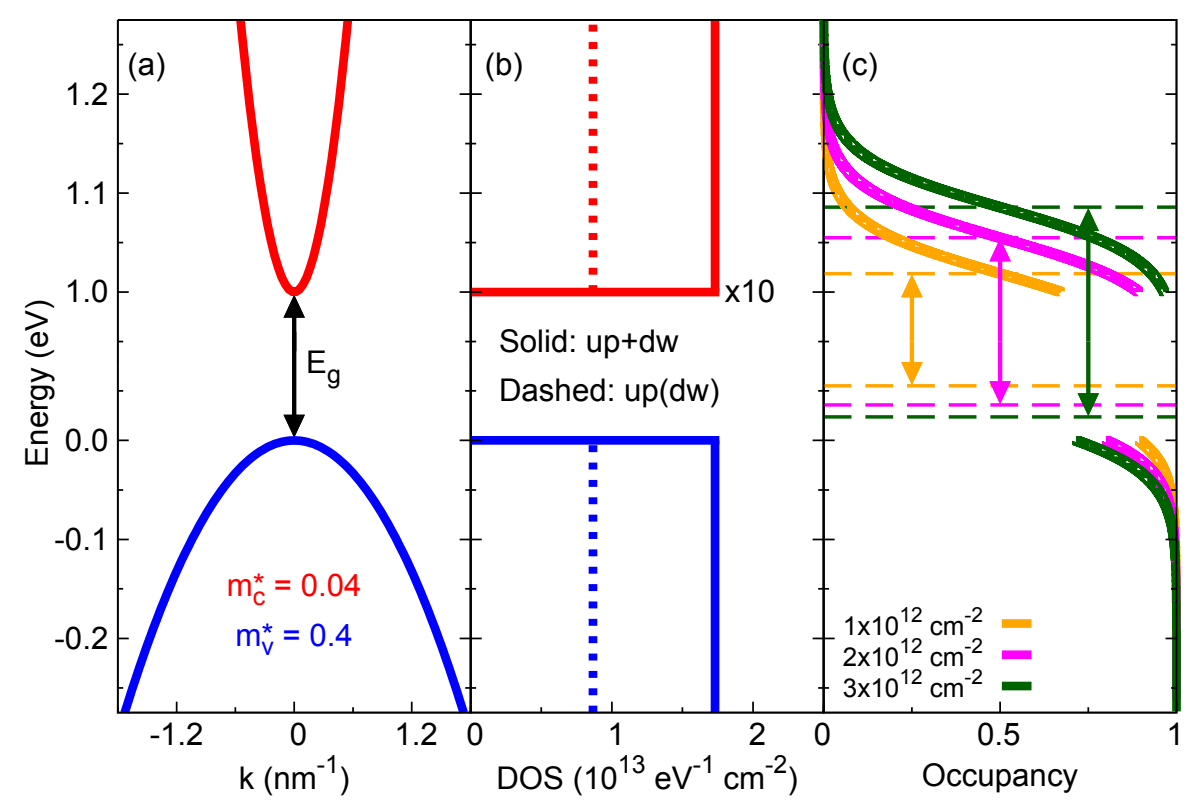

Figure 3.2 - (a) Band structure, (b) DOS and (c) Fermi-Dirac occupancy without spin polarization for the two-band model. We used $m_{c}^{*}=0.04, m_{v}^{*}=0.4$ and $E_{g}=1.0 \mathrm{eV}$. For the occupancy we assumed $T=300 \mathrm{~K}$. Vertical arrows indicate (a) the energy gap and (b) the differences of chemical potential energies, $\Delta \mu=\mu_{C}-\mu_{V}$.

Source: By the author.

difference between conduction and valence band chemical potentials, $\Delta \mu$, exceeds the value of the energy gap, $E_{g}$. Therefore, for photon energies in the range $E_{g}<\hbar \omega<\Delta \mu$ we have the lasing regime. The crossing point from lasing to absorption regime is exactly at $\hbar \omega=\Delta \mu$. It is important to note that, without spin polarization, the gain coefficient is the same for $S^{+}$and $S^{-}$ light polarizations.

Let us now turn to the case with spin polarized carriers, illustrated by the dashed lines in Fig. 3.3(b). For this investigation, we assume only electrons to be spin polarized with the value of $P_{c}=0.5$. We show that the gain coefficients for $S^{+}$and $S^{-}$provide uneven contributions, which, in this simple case, is mainly ruled by the different occupancies of electron spins in the conduction band. Similarly to the case without spin polarization, the photon energy must satisfy the condition $E_{g}<\hbar \omega<\Delta \mu_{\uparrow(\downarrow)}$, however, this energy range is now different for each light polarization. For instance, a carrier density of $2 \times 10^{12} \mathrm{~cm}^{-2}$ added to the system, allows only $S^{+}$polarized light to be in the lasing regime. Increasing the carrier density to $3 \times 10^{12} \mathrm{~cm}^{-2}$, both light polarizations achieve the lasing condition. 


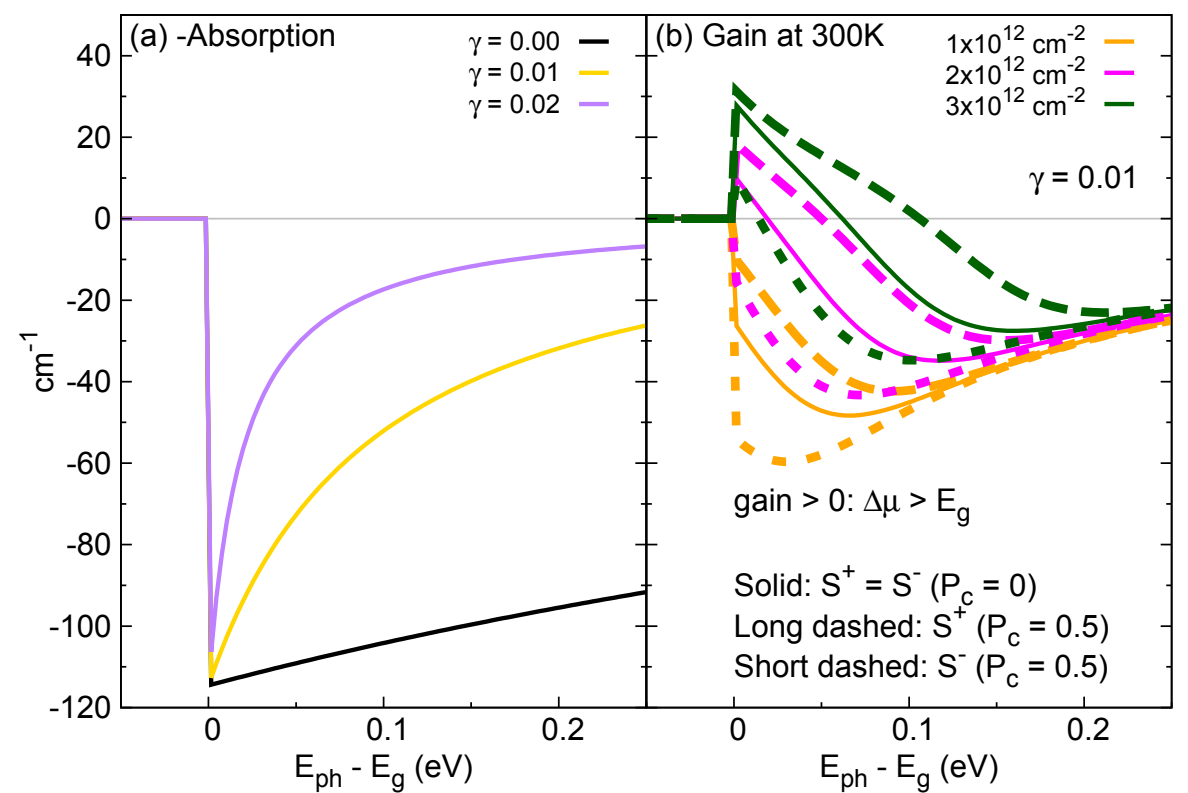

Figure 3.3 - (a) Gain coefficient at $T=0 \mathrm{~K}$, i. e., the negative value of the absorption, without injected carriers and (b) gain coefficient at $T=300 \mathrm{~K}$ with and without spin polarized electrons for different carrier densities. Different values of $\gamma$ are used in (a). In (b), we fixed $\gamma=0.01$ and considered different values of carrier densities. For the constant $C_{0}$ [equation (3.35)], we used $P=10 \mathrm{eV} \AA, L=80 \AA$ and $n_{r}=3$.

Source: By the author.

Although this simple model does not capture all the band structure features of more complex systems, such as quantum wells with several subbands, it provides a physical insight for microscopic behavior of the spin-dependent gain. Based on these gain coefficient calculations, we can systematically investigate the operation of spin lasers from a microscopic point of view under different carrier injection and cavity design conditions. In the next chapter, specially sections 4.1 and 4.3, we investigate absorption and gain coefficients in more realistic systems such as GaAs quantum well spin lasers and polytypic ZB/WZ InP superlattices. 
4 Papers 


\title{
4.1 Toward high-frequency operation of spin lasers
}

\author{
$\underline{\text { Paulo E. Faria Junior }}$
}

Department of Physics, University at Buffalo, State University of New York, Buffalo, New York 14260, USA Instituto de Física de São Carlos, Universidade de São Paulo, 13566-590 São Carlos, São Paulo, Brazil

\section{Gaofeng Xu}

Department of Physics, University at Buffalo, State University of New York, Buffalo, New York 14260, USA

\section{Jeongsu Lee}

Department of Physics, University at Buffalo, State University of New York, Buffalo, New York 14260, USA Institute for Theoretical Physics, University of Regensburg, 93040 Regensburg, Germany

Nils C. Gerhardt

Department of Physics, University at Buffalo, State University of New York, Buffalo, New York 14260, USA Photonics and Terahertz Technology, Ruhr-University Bochum, D-44780 Bochum, Germany

\section{Guilherme M. Sipahi}

Department of Physics, University at Buffalo, State University of New York, Buffalo, New York 14260, USA Instituto de Física de São Carlos, Universidade de São Paulo, 13566-590 São Carlos, São Paulo, Brazil

\section{Igor Žutić}

Department of Physics, University at Buffalo, State University of New York, Buffalo, New York 14260, USA 


\title{
Toward high-frequency operation of spin lasers
}

\author{
Paulo E. Faria Junior, ${ }^{1,2}$ Gaofeng Xu, ${ }^{1}$ Jeongsu Lee, ${ }^{1,3}$ Nils C. Gerhardt, ${ }^{1,4}$ Guilherme M. Sipahi, ${ }^{1,2}$ and Igor Žutić1 ${ }^{, *}$ \\ ${ }^{1}$ Department of Physics, University at Buffalo, State University of New York, Buffalo, New York 14260, USA \\ ${ }^{2}$ Instituto de Física de São Carlos, Universidade de São Paulo, 13566-590 São Carlos, São Paulo, Brazil \\ ${ }^{3}$ Institute for Theoretical Physics, University of Regensburg, 93040 Regensburg, Germany \\ ${ }^{4}$ Photonics and Terahertz Technology, Ruhr-University Bochum, D-44780 Bochum, Germany
}

(Received 23 May 2015; published 25 August 2015)

\begin{abstract}
Injecting spin-polarized carriers into semiconductor lasers provides important opportunities to extend what is known about spintronic devices, as well as to overcome many limitations of conventional (spin-unpolarized) lasers. By developing a microscopic model of spin-dependent optical gain derived from an accurate electronic structure in a quantum-well-based laser, we study how its operation properties can be modified by spin-polarized carriers, carrier density, and resonant cavity design. We reveal that by applying a uniaxial strain, it is possible to attain a large birefringence. While such birefringence is viewed as detrimental in conventional lasers, it could enable fast polarization oscillations of the emitted light in spin lasers, which can be exploited for optical communication and high-performance interconnects. The resulting oscillation frequency $(>200 \mathrm{GHz})$ would significantly exceed the frequency range possible in conventional lasers.
\end{abstract}

DOI: 10.1103/PhysRevB.92.075311

PACS number(s): 42.55.Px, 78.45.+h, 78.67.De, 78.67.Hc

\section{INTRODUCTION}

Both spin lasers and their conventional (spin-unpolarized) counterparts share three main elements: (i) the active (gain) region, responsible for optical amplification and stimulated emission, (ii) the resonant cavity, and (iii) the pump, which injects (optically or electrically) energy/carriers. The main distinction of spin lasers is a net carrier spin polarization (spin imbalance) in the active region, which, in turn, can lead to crucial changes in their operation, as compared to their conventional counterparts. This spin imbalance is responsible for a circularly polarized emitted light, a result of the conservation of the total angular momentum during electron-hole recombination [1].

The experimental realization of spin lasers [2-19] presents two important opportunities. The lasers provide a path to practical room-temperature spintronic devices with different operating principles, not limited to magnetoresistive effects, which have enabled tremendous advances in magnetically stored information [20-24]. This requires revisiting the common understanding of material parameters for desirable operation [25], as well as a departure from more widely studied spintronic devices, where only one type of carrier (electrons) plays an active role. In contrast, since semiconductor lasers are bipolar devices, a simultaneous description of electrons and holes is crucial.

On the other hand, the interest in spin lasers is not limited to spintronics, as they may extend the limits of what is feasible with conventional semiconductor lasers. It was experimentally demonstrated that injecting spin-polarized carriers already leads to noticeable differences in the steady-state operation [4-6]. The onset of lasing is attained for a smaller injection lasing threshold reduction, while the optical gain differs for different polarizations of light, leading to gain asymmetry, also referred to as gain anisotropy $[5,6,8]$. In the stimulated emission, even a small carrier polarization in the active region

*zigor@buffalo.edu can be greatly amplified and lead to the emission of completely circularly polarized emitted light, an example of a very efficient spin filtering [13].

An intuitive picture for a spin laser is provided by a bucket model in Fig. 1 [26,27]. The uneven water levels represent the spin imbalance in the laser, which implies the following: (i) Lasing threshold reduction-in a partitioned bucket, less water needs to be pumped for it to overfill. There are also two thresholds (for cold and hot water) [28]. (ii) Gain asymmetry - an unequal amount of hot and cold water comes out. A small spin imbalance of pumped carriers can (the two water levels slightly above and below the opening, respectively) result in a complete imbalance in the polarization of the emitted light (here only hot water gushes out) and, consequently, spin-filtering. These effects are attained at room

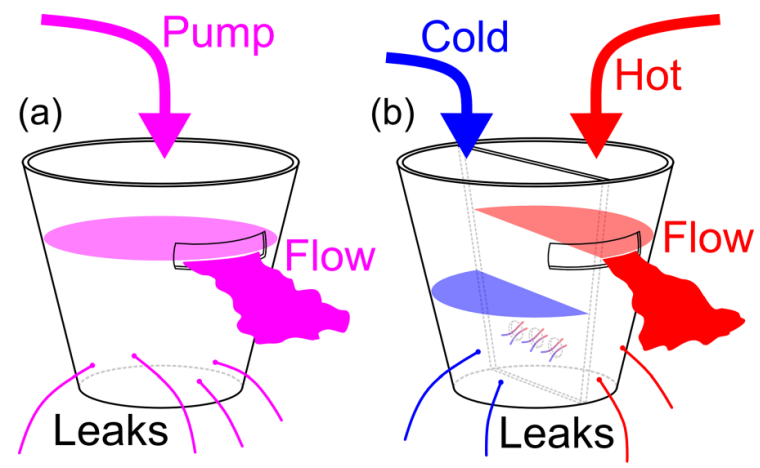

FIG. 1. (Color online) Bucket model for (a) a conventional laser and (b) a spin laser [26]. Water added to the bucket represents the carriers, and the water coming out represents the emitted light. Small leaks depict spontaneous emission, and overflowing water reaching the large opening corresponds to the lasing threshold. In (b) the two halves represent two spin populations (hot and cold water in the analogy), and they are filled separately. The partition between them is not perfect: spin relaxation can cause the two populations to mix. The color code indicates conservation of angular momentum; an unpolarized pumping (violet) is an equal mixture of two polarized contributions (red and blue). 
temperature with either optical or electrical injection. The latter experimental demonstration [17] is a breakthrough toward practical use of spin lasers.

Perhaps the most promising opportunity to overcome the limitations of conventional lasers lies in the dynamic operation of spin lasers, predicted to provide enhanced modulation bandwidth, improved switching properties, and reduced parasitic frequency modulation, i.e., chirp [25,26,29,30]. Moreover, experiments have confirmed that in a given device, a characteristic frequency of polarization oscillations of the emitted light can significantly exceed the corresponding frequency of the intensity oscillations $[11,12,16]$. This behavior was attributed to birefringence-an anisotropy of the index of refraction, considered detrimental in conventional lasers [31].

What should we then require to attain high-frequency operation in spin lasers? Can we provide guidance for the design of an active region and a choice of the resonant cavity? Unfortunately, to address similar questions, we cannot simply rely on the widely used rate-equation description of spin-lasers $[4,5,26,32,33]$, but instead we need to formulate a microscopic description. The crucial consideration is detailed knowledge of the spectral (energy-resolved) optical gain obtained from an accurate description of the electronic structure in the active region, already important to elucidate a steady-state operation of a spin laser.

A typical vertical geometry, the so-called vertical cavity surface emitting lasers (VCSELs) [31,34-36], used in nearly all spin lasers, is illustrated in Fig. 2(a). Even among conventional lasers, VCSELs are recognized for their unique properties, making them particularly suitable for optical data transmission [36]. The resonant cavity is usually in the range of the emission wavelength, providing a longitudinal single-mode operation. It is formed by a pair of parallel highly reflective mirrors made of distributed Bragg reflectors (DBRs), a layered structure with varying refractive index. The gain active (gain) region usually consists of III-V quantum wells (QWs) or quantum dots (QDs) [7-9,26,37-39].

The key effect of the active region is producing a stimulated emission and coherent light that makes the laser

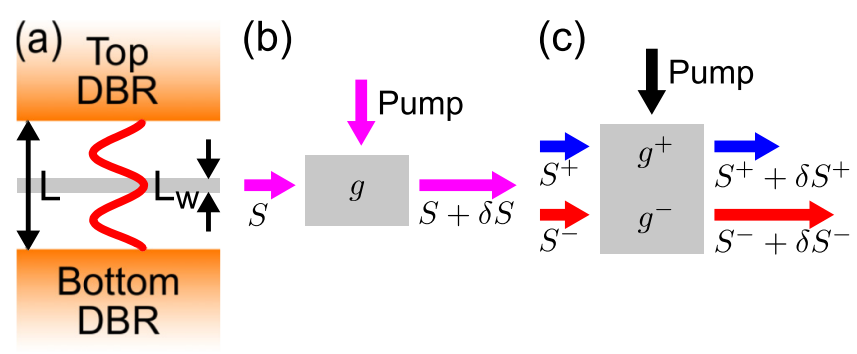

FIG. 2. (Color online) (a) Geometry of a vertical cavity surfaceemitting laser. The resonant cavity of length $L$ is formed between the two mirrors made of distributed Bragg reflectors (DBRs). The shaded region represents the active (gain) region of length $L_{W}$. The profile of a longitudinal optical mode is sketched. Schematic of the optical gain, $g$, in the active region for a conventional laser (b) and a spin laser (c). With external pumping/injection, a photon density $S$ increases by $\delta S$ as it passes across the gain region [40]. In the spin laser, this increase depends on the positive $(+) /$ negative $(-)$ helicity of the light, $S=S^{+}+S^{-}$. such a unique light source. The corresponding optical gain that describes stimulated emission, under sufficiently strong pumping/injection of carriers, can be illustrated pictorially in Figs. 2(b) and 2(c) for both conventional and spin lasers, respectively. In the latter case, it is convenient to decompose the photon density into different circular polarizations and distinguish that the gain is generally polarization-dependent. If we neglect any losses in the resonant cavity, such a gain would provide an exponential growth rate with the distance across a small segment of gain material [34]. Since both static and dynamic operations of spin lasers depend crucially on their corresponding optical gain, our focus will be to provide its microscopic description derived from an accurate electronic structure of an active region.

After this Introduction, in Sec. II we provide a theoretical framework to calculate the gain in quantum-well-based lasers. In Sec. III, we describe the corresponding electronic structure and the carrier populations under spin injection, the key prerequisites to understanding the spin-dependent gain and its spectral dependence, discussed in Sec. IV. Our gain calculations in Sec. V explain how the steady-state properties of spin lasers can be modified by spin-polarized carriers, carrier density, and resonant cavity design. In Sec. VI, we analyze the influence of a uniaxial strain in the active region, which introduces a large birefringence with the resulting oscillation frequency that would significantly exceed the frequency range possible in conventional lasers. In Sec. VII, we describe various considerations for the optimized design of spin lasers and the prospect of their ultrahigh-frequency operation. A brief summary in Sec. VIII ends our paper.

\section{THEORETICAL FRAMEWORK}

While both QWs and QDs [7-9] are used for the active region of spin lasers, we focus here on the QW implementation also found in most of the commercial VCSELs [36]. To obtain an accurate electronic structure in the active region, needed to calculate optical gain, we use the $8 \times 8 \boldsymbol{k} \cdot \boldsymbol{p}$ method [41] The total Hamiltonian of the QW system, with the growth axis along the $z$ direction, is

$$
H_{\mathrm{QW}}(z)=H_{\boldsymbol{k} \cdot \boldsymbol{p}}(z)+H_{\mathrm{st}}(z)+H_{\mathrm{O}}(z),
$$

where $H_{\boldsymbol{k} \cdot \boldsymbol{p}}(z)$ denotes the $\boldsymbol{k} \cdot \boldsymbol{p}$ term, $H_{\mathrm{st}}(z)$ describes the strain term, and $H_{\mathrm{O}}(z)$ includes the band offset at the interface that generates the QW energy profile. The explicit form of these different terms for zinc-blende crystals is given in Appendix A.

Because common nonmagnetic semiconductors are well characterized by the vacuum permeability, $\mu_{0}$, a complex dielectric function $\varepsilon(\omega)=\varepsilon_{r}(\omega)+\varepsilon_{i}(\omega)$, where $\omega$ is the photon (angular) frequency, can be used to simply express the dispersion and absorption of electromagnetic waves. The absorption coefficient describing gain or loss of the amplitude of an electromagnetic wave propagating in such a medium is the negative value of the gain coefficient (or gain spectrum) $[31,42,43]$,

$$
g^{a}(\omega)=-\frac{\omega}{c n_{r}} \epsilon_{i}^{a}(\omega),
$$

where $c$ is the speed of light, $n_{r}$ is the dominant real part of the refractive index of the material [42], and $\varepsilon_{i}^{a}(\omega)$ is the imaginary 
part of the dielectric function, which generally depends on the polarization of light, $a$, given by

$$
\varepsilon_{i}^{a}(\omega)=C_{0} \sum_{c, v, \vec{k}}\left|p_{c v \vec{k}}^{a}\right|^{2}\left(f_{v \vec{k}}-f_{c \vec{k}}\right) \delta\left[\hbar \omega_{c v \vec{k}}-\hbar \omega\right],
$$

where the indices $c$ (not to be confused with the speed of light) and $v$ label the conduction and valence subbands, respectively, $\vec{k}$ is the wave vector, $p_{c v \vec{k}}^{a}$ is the interband dipole transition amplitude, $f_{c(v) \vec{k}}$ is the Fermi-Dirac distribution for the electron occupancy in the conduction (valence) subbands, $\hbar$ is Planck's constant, $\omega_{c v \vec{k}}$ is the interband transition frequency, and $\delta$ is the Dirac delta function, which is often replaced to include broadening effects for finite lifetimes [31,44]. The constant $C_{0}$ is $C_{0}=4 \pi^{2} e^{2} /\left(\varepsilon_{0} m_{0}^{2} \omega^{2} \Omega\right)$, where $e$ is the electron charge, $m_{0}$ is the free-electron mass, and $\Omega$ is the $\mathrm{QW}$ volume.

Analogously to expressing the total photon density in Fig. 2, as the sum of different circular polarizations, $S=S^{+}+S^{-}$, in spin-resolved quantities we use subscripts to describe different spin projections, i.e., eigenvalues of the $\sigma_{z}$ Pauli matrix. The total electron/hole density can be written as the sum of the spin-up (+) and spin-down (-) electron/hole densities, $n=$ $n_{+}+n_{-}$and $p=p_{+}+p_{-}$. In this convention $[25,28,29]$, using the conservation of angular momentum between carriers and photons, the recombination terms are $n_{+} p_{+}, n_{-} p_{-}$, while the corresponding polarization of the emitted light depends on the character of the valence-band holes [45]. We can simply define the carrier spin polarization

$$
P_{\alpha}=\left(\alpha_{+}-\alpha_{-}\right) /\left(\alpha_{+}+\alpha_{-}\right),
$$

where $\alpha=n, p$ [46].

Using the dipole selection rules for the spin-conserving interband transitions, the gain spectrum,

$$
g^{a}(\omega)=g_{+}^{a}(\omega)+g_{-}^{a}(\omega),
$$

can be expressed in terms of the contributions of spin-up and -down carriers. To obtain $g_{+(-)}^{a}(\omega)$, the summation of conduction and valence subbands is restricted to only one spin: $\sum_{c} \rightarrow \sum_{c+(-)}$ and $\sum_{v} \rightarrow \sum_{v+(-)}$ in Eq. (3).

To see how spin-polarized carriers could influence the gain, we show chemical potentials, $\mu_{C(V)}$, for a simplified conduction (valence) band in Fig. 3(a). The spin imbalance in the active region implies that $\mu_{C(V)}$ will also become spin-dependent. Such different chemical potentials lead to the dependence of gain on the polarization of light, described in Fig. 3(b). Without spin-polarized carriers, the gain is the same for $S^{+}$and $S^{-}$helicity of light. In an ideal semiconductor laser, $g>0$ requires a population inversion for photon energies, $E_{g}<\hbar \omega<\left(\mu_{C}-\mu_{V}\right)$. However, a gain broadening is inherent to lasers and, as depicted in Fig. 3(b), $g>0$ even below the band gap, $\hbar \omega<E_{g}$. If we assume $P_{n} \neq 0$ [recall Eq. (4)] and $P_{p}=0$ (accurately satisfied, as spins of holes relax much faster than electrons), we see different gain curves for $S^{+}$and $S^{-}$. The crossover from emission to absorption is now in the range of $\left(\mu_{C_{-}}-\mu_{V_{-}}\right)$and $\left(\mu_{C+}-\mu_{V+}\right)$.

Optical injection of spin-polarized electrons is the most frequently used method to introduce spin imbalance in lasers. Some spin lasers are therefore readily available since they can be based on commercial semiconductor lasers to which a source of circularly polarized light is added subsequently
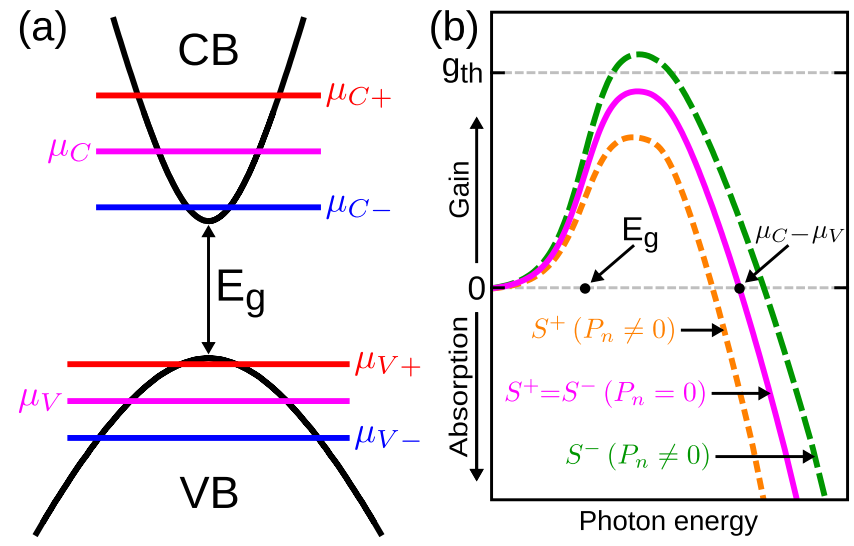

FIG. 3. (Color online) (a) Energy band diagram with a band gap $E_{g}$ and chemical potentials in conduction (valence) bands, $\mu_{C}\left(\mu_{V}\right)$, that in the presence of spin-polarized carriers become spin-dependent: $\mu_{C(V)+} \neq \mu_{C(V)-}$. Unlike the rest of our analysis, here holes are spin-polarized. (b) Gain spectrum for unpolarized (solid) and spinpolarized electrons (dashed curves). Positive gain corresponds to the emission and negative gain to the absorption of photons. The gain threshold $g_{\text {th }}$, required for lasing operation, is attained only for $S^{-}$ helicity of light.

[4]. Such spin injection can be readily understood from dipole optical selection rules, which apply for both excitation and radiative recombination $[1,20]$.

A simplified band diagram for a zinc-blende QW semiconductor with the corresponding interband transitions is depicted in Fig. 4. At the Brillouin zone center, the valence-band degeneracy of heavy and light holes (HH,LH) in the bulk semiconductor is lifted for QWs due to quantum confinement along the growth direction. The angular momentum
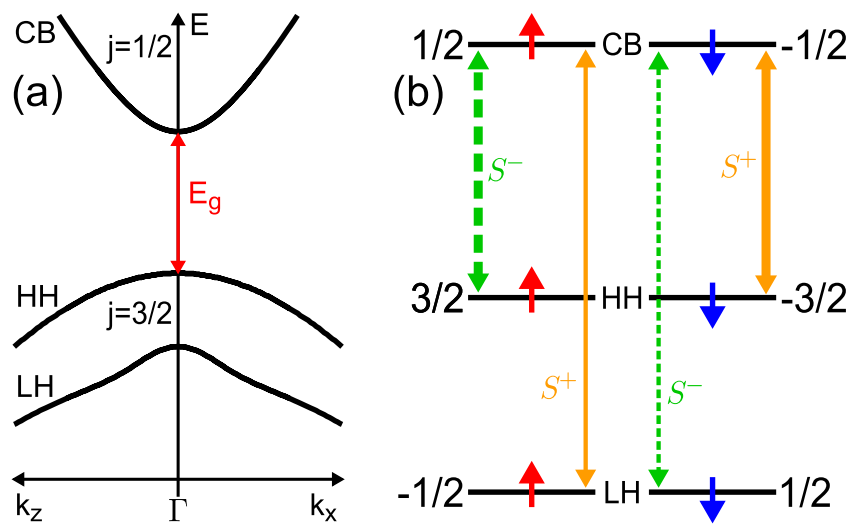

FIG. 4. (Color online) Schematic band structure and optical selection rules in zinc-blende QWs. (a) Conduction band (CB) and valence band with heavy and light holes $(\mathrm{HH}, \mathrm{LH})$ labeled by their total angular momentum $j=1 / 2$ and $3 / 2$, representing the states of the orbital angular momentum $l=0$ and 1 , respectively (Appendix A). (b) Interband dipole transitions near the band edge of a QW for light with positive and negative helicity, $S^{ \pm}$, between the sublevels labeled by $m_{j}$, the projection of the total angular momentum on the $+z$ axis (along the growth direction). The small arrows represent the projection of spin $1 / 2$ of the orbital part that contributes to the transition, indicating that dipole transitions do not change spin (Appendix B). 
of absorbed circularly polarized light is transferred to the semiconductor. Electron orbital momenta are directly oriented by light, and, through spin-orbit interaction, their spins become polarized [1]. While initially holes are also polarized, their spin polarization is quickly lost [20]. Thus, as in Fig. 3(b), we assume throughout this work $P_{p}=0$, unless stated otherwise.

The spin polarization of excited electrons depends on the photon energy for $S^{+}$or $S^{-}$light. From Fig. 4(b) we can infer that if only CB-HH are involved, $\left|P_{n}\right| \rightarrow 1$. At a larger $\hbar \omega$, involving also CB-LH transitions, $\left|P_{n}\right|$ is reduced. Expressing $S^{ \pm} \propto Y_{1}^{ \pm 1}$, where $Y_{l}^{m}$ is the spherical harmonic, provides a simple connection between dipole selection rules and the conservation of angular momentum in optical transitions (Appendix B).

\section{ELECTRONIC STRUCTURE}

For our microscopic description of spin lasers, we focus on an $(\mathrm{Al}, \mathrm{Ga}) \mathrm{As} / \mathrm{GaAs}$-based active region, a choice similar to many commercial VCSELs. We consider an $\mathrm{Al}_{0.3} \mathrm{Ga}_{0.7}$ As barrier and a single 8-nm-thick GaAs QW [47]. The corresponding electronic structure of both the band dispersion and the density of states (DOS) is shown in Fig. 5. Our calculations, based on the $\boldsymbol{k} \cdot \boldsymbol{p}$ method and the $8 \times 8$ Hamiltonian from Eq. (1) (Appendix A), yield two confined CB subbands: CB1, CB2, and five VB subbands, labeled in Fig. 5(a) by the dominant component of the total envelope function at $\vec{k}=0$. The larger number of confined VB subbands stems from larger effective masses for holes than electrons [48]. These differences in the effective masses also appear in the DOS shown in Fig. 5(b).

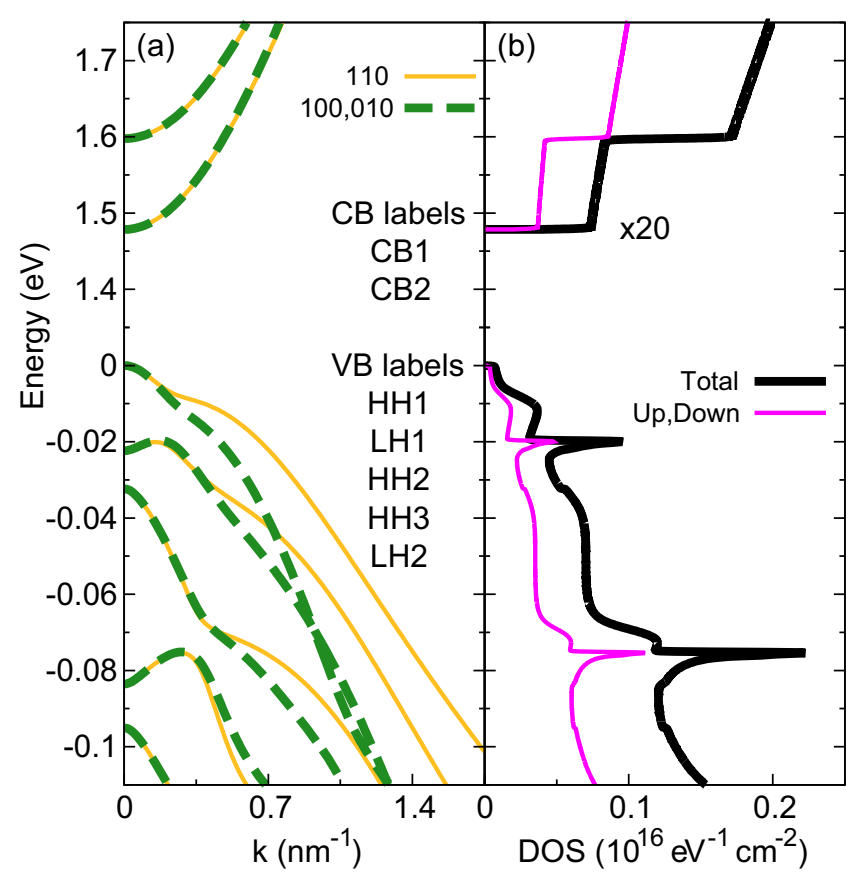

FIG. 5. (Color online) (a) Band structure for the $\mathrm{Al}_{0.3} \mathrm{Ga}_{0.7} \mathrm{As} /$ GaAs QW for different $k$ directions along [100], [010], and [110]. (b) DOS calculated from (a). The conduction-band DOS is multiplied by a factor of 20 to match the valence-band scale. The band gap is $E_{g}=1.479 \mathrm{eV}$ (CB1-HH1 energy difference).

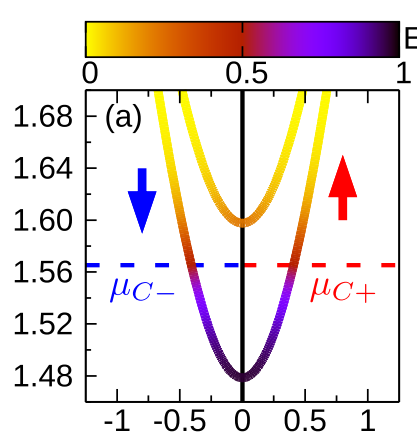
Electron occupancy
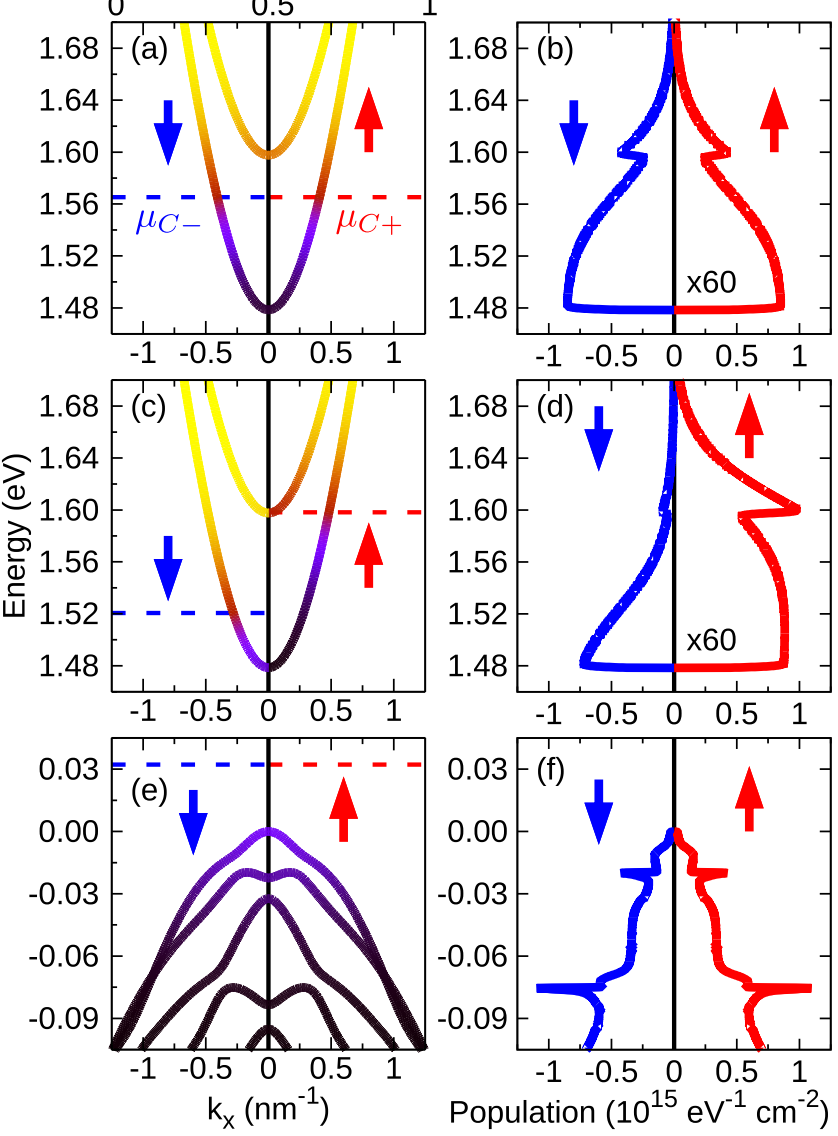

FIG. 6. (Color online) Band structure of Fig. 5(a) with electron occupancy for (a) $P_{n}=0$, (c) $P_{n}=0.5$, and (e) $P_{p}=0$. Carrier population expressed as a product of DOS from Fig. 5(b) and the Fermi-Dirac distribution of electrons for (b) $P_{n}=0$, (d) $P_{n}=0.5$, and (f) $P_{p}=0$. The carrier density is fixed at $n=p=3 \times 10^{12} \mathrm{~cm}^{-2}$ and $T=300 \mathrm{~K}$. The negative (positive) side of the $x$ axis represents spin-down (-up) electrons, dashed lines denote chemical potentials. The CB population is multiplied by 60 and shown in the same scale as for the VB.

As we seek to describe the gain spectrum in the active region, once we have the electronic structure, it is important to understand the effects associated with carrier occupancies though injection/pumping [recall Fig. 2, Eqs. (2) and (3)]. In Figs. 6(a), 6(c), and 6(e), we show both examples of injected unpolarized $\left(P_{n}=0\right)$ and spin-polarized $\left(P_{n}=0.5\right)$ electrons as seen in the equal and spin-split $\mathrm{CB}$ chemical potentials, respectively. The carrier population [34] is given in Figs. 6(b), 6(d), and 6(f) using the product of the Fermi-Dirac distribution and the DOS for CB and VB for both spin projections.

\section{UNDERSTANDING THE SPIN-DEPENDENT GAIN}

From the conservation of angular momentum and polarization-dependent optical transitions, we can understand that even in conventional lasers, carrier spin plays a role in determining the gain. However, in the absence of spin-polarized carriers [49], the gain is identical for the two helicities: $g^{+}=g^{-}$, and we recover a simple description (spin- and 

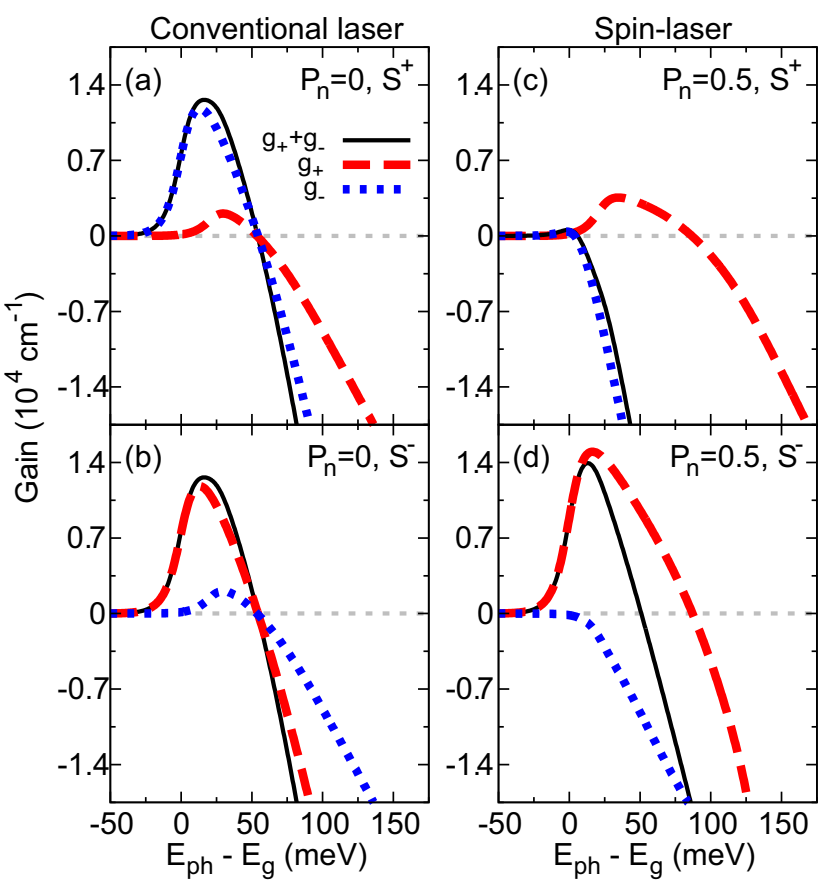

FIG. 7. (Color online) Gain spectra shown as a function of photon energy measured with respect to the energy band gap. Conventional laser, $P_{n}=0$ for (a) $S^{+}$and (b) $S^{-}$light polarization. Spin-lasers, $P_{n}=0.5$ for (c) $S^{+}$and (d) $S^{-}$light polarization. The carrier density $n=p=3 \times 10^{12} \mathrm{~cm}^{-2}$ and $T=300 \mathrm{~K}$ are the same as in Fig. 6.

polarization-independent) from Fig. 2(b). In our notation, $g_{ \pm}^{ \pm}$, the upper (lower) index refers to the circular polarization (carrier spin) [recall Eq. (5)].

This behavior can be further understood from the gain spectrum in Figs. 7(a) and 7(b), where we recognize that $g^{+}=g^{-}$requires (i) $g_{-}^{+}=g_{+}^{-}$and $g_{+}^{+}=g_{-}^{-}$, dominated by CB1-HH1 $\left(1.479 \mathrm{eV}=E_{g}\right)$ and CB1-LH1 $(1.501 \mathrm{eV})$ transitions, respectively (recall Fig. 5). No spin imbalance implies spin-independent $\mu_{C}$ and $\mu_{V}$ [Fig. 3(a)], and thus $g^{ \pm}, g_{+}^{ \pm}$, and $g_{-}^{ \pm}$all vanish the photon energy $\mathrm{E}_{\mathrm{ph}}=\hbar \omega=$ $\mu_{C}-\mu_{V}$. Throughout our calculations, we choose a suitable $\cosh ^{-1}$ broadening [44] with a full width at half-maximum (FWHM) of $19.75 \mathrm{meV}$, which accurately recovers the gain of conventional $(\mathrm{Al}, \mathrm{Ga}) \mathrm{As} / \mathrm{GaAs} \mathrm{QW}$ systems.

We next turn to the gain spectrum of spin lasers. Why is their output different for $S^{+}$and $S^{-}$light, as depicted in Fig. 2(b)? Changing only $P_{n}=0.5$ from Figs. 7(a) and 7(b), we see very different results for $S^{+}$and $S^{-}$light in Figs. 7(c) and 7(d). $P_{n}>0$ implies that $\mu_{C+}>\mu_{C-}$ [see Fig. 6(c)], leading to a larger recombination between the spin-up carriers $\left(n_{+} p_{+}>\right.$ $n_{-} p_{-}$) and thus to a larger $g_{+}$for $S^{+}$and $S^{-}$(red/dashed line) than $g_{-}$(blue/dashed line). The combined effect of having spin-polarized carriers and different polarization-dependent optical transitions for spin-up and -down recombination is then responsible for $g^{+} \neq g^{-}$, given by solid lines in Figs. 7(c) and $7(\mathrm{~d})$. For this case, the emitted light $S^{-}$exceeds that with the opposite helicity, $S^{+}$, and there is a gain asymmetry $[5,6,8]$, another consequence of the polarization-dependent gain. The zero gain is attained at $\mu_{C+}-\mu_{V}$ for spin-up (red curves) and
$\mu_{C-}-\mu_{V}$ for spin-down transitions (blue curves). The total gain, including both of these contributions, reaches zero at an intermediate value. Without any changes to the band structure, a simple reversal of the carrier spin polarization, $P_{n} \rightarrow-P_{n}$, reverses the role of preferential light polarization.

\section{STEADY-STATE GAIN PROPERTIES}

Within our framework, providing spectral information for the gain, we can investigate how the carrier density and its spin polarization, which can be readily modified experimentally, can change the steady-state operation of spin lasers. Specific to VCSELs, it is important to examine how their laser operation is

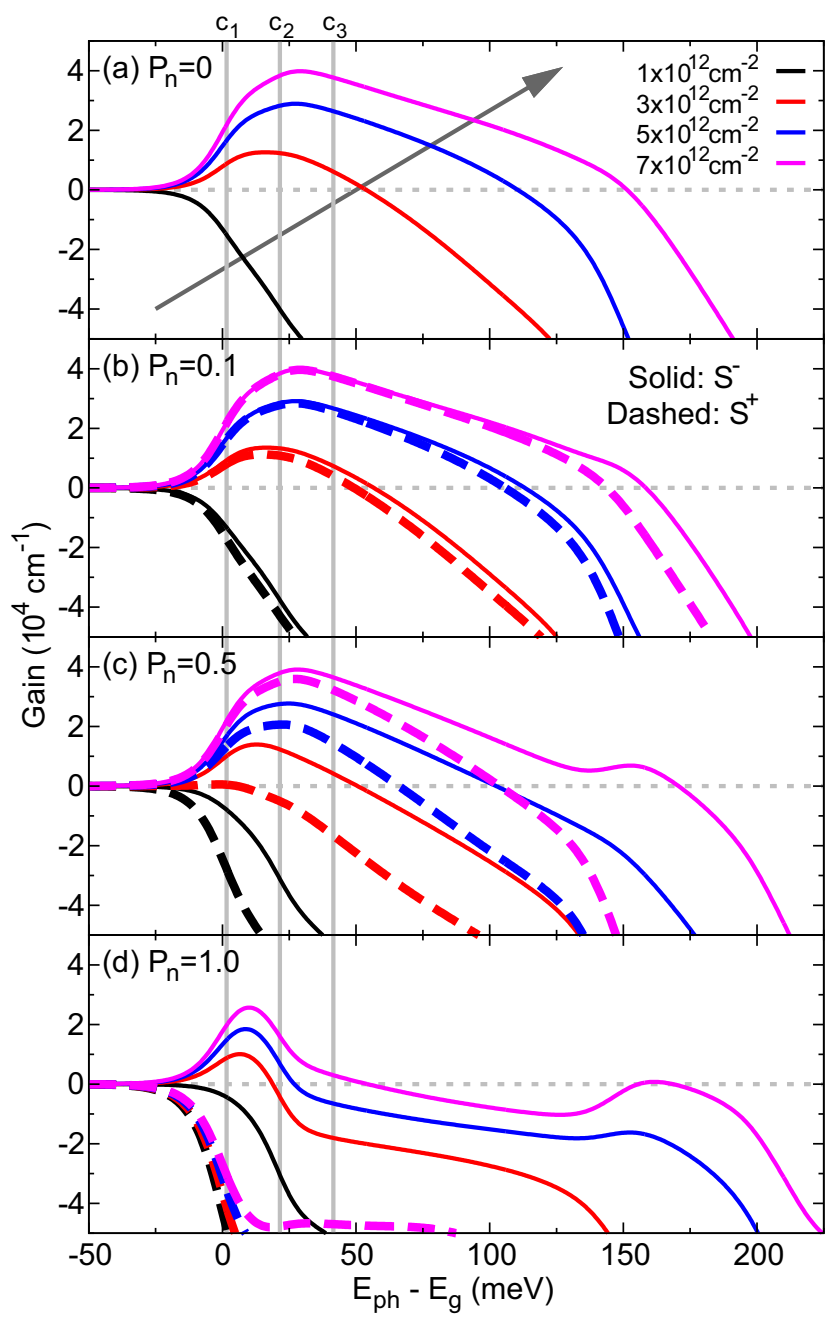

FIG. 8. (Color online) Evolution of the gain spectra with carrier density for (a) $P_{n}=0$, (b) $P_{n}=0.1$, (c) $P_{n}=0.5$, and (d) $P_{n}=1.0$. To achieve emission, a certain value of carrier density should be added to the system. The second peak at $E_{\mathrm{ph}}-E_{\mathrm{g}} \sim 150 \mathrm{meV}$ is related to transitions of CB2-HH2. Transitions of CB2-LH2 at $E_{\mathrm{ph}}-E_{\mathrm{g}} \sim$ $200 \mathrm{meV}$ can be seen in the broader second peak for $P_{n}=1.0$. The difference between $g^{+}$and $g^{-}$that arises due to spin-polarized carriers in the system increases with $P_{n}$. For $P_{n}=1.0$ there is no emission of $S^{+}$-polarized light, i.e., this component is totally absorbed by the system. The diagonal arrow in Fig. 8 indicates the increase of carrier density in the curves. 


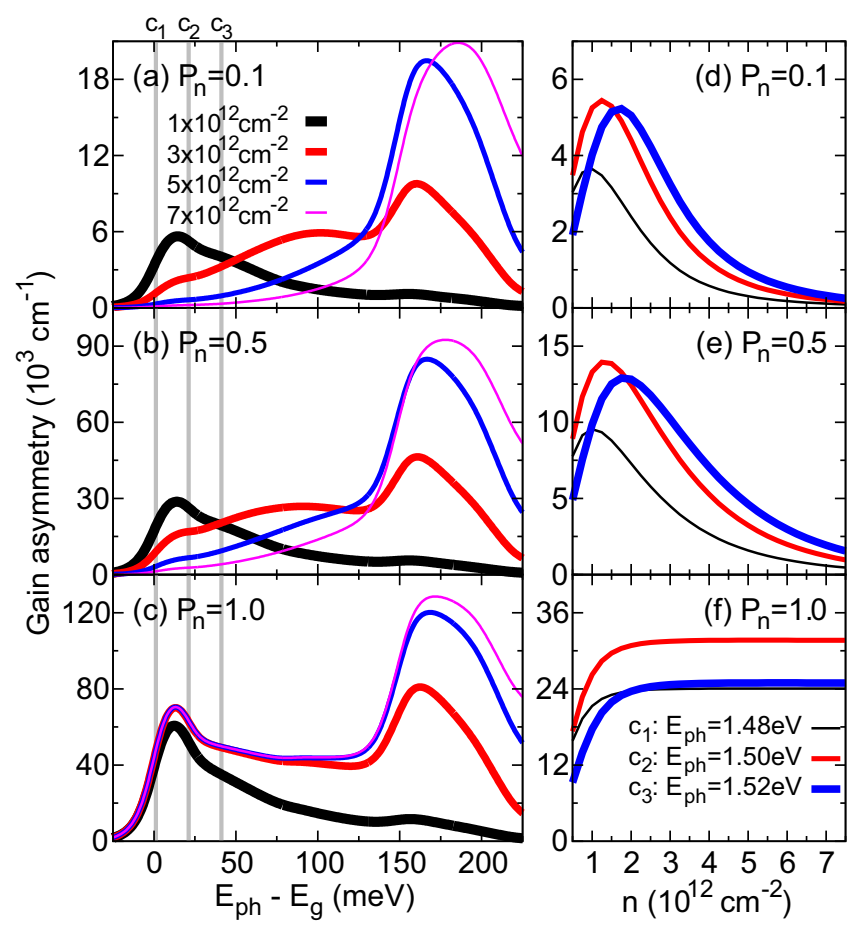

FIG. 9. (Color online) Gain asymmetry obtained from Fig. 8 for (a) $P_{n}=0.1$, (b) $P_{n}=0.5$, and (c) $P_{n}=1.0$. As more carriers are added to the system, the asymmetry peak shifts to higher energies, however this energy region is not necessarily in the regime of a positive gain. Gain asymmetry as a function of carrier density for (d) $P_{n}=0.1$, (e) $P_{n}=0.5$, and (f) $P_{n}=1.0$. Similar to the case of (a)-(c), the asymmetry peaks may not correspond to positive gain.

related to the choice of a resonant cavity, which defines the photon energy at which the constructive interference takes place.

Most of the QW-based lasers do not have a doped active region, and they rely on a charge neutral carrier injection (electrical or optical) [34]. Here we choose $n=p=1,3$, 5 , and $7 \times 10^{12} \mathrm{~cm}^{-2}$, and spin polarizations $P_{n}=0,0.1$, 0.5 , and 1 , respectively. Electrical injection in intrinsic III-V QWs using $\mathrm{Fe}$ or FeCo allows for $\left|P_{n}\right| \sim 0.3-0.7$ [50-52], while $\left|P_{n}\right| \rightarrow 1$ is attainable optically at room temperature [20]. In most of the spin lasers, $\left|P_{n}\right| \lesssim 0.2$ in the active region. We focus on three resonant cavity positions: $c_{1}, c_{2}, c_{3}$ (vertical lines), defining the corresponding energy of emitted photons $c_{1}=1.48 \sim 1.479 \mathrm{eV}$ (CB1-HH1 transition), $c_{2}=$ $1.50 \sim 1.501 \mathrm{eV}$ (CB1-LH1 transition), and $c_{3}=1.52 \mathrm{eV}$ (at the high-energy side of the gain spectrum).

The corresponding results are given in Fig. 8 showing gain spectra different for $S^{+}$and $S^{-}$. This gain asymmetry, $g^{+} \neq g^{-}$, is more pronounced at larger $P_{n}$; at $P_{n}=1$, there is even no $S^{+}$emission. While this trend is expected and could be intuitively understood, there is a more complicated dependence of the gain asymmetry, $g^{-}(\hbar \omega)-g^{+}(\hbar \omega)$, on the carrier density, and the choice of the detuning [42], the energy (frequency) difference between the gain peak, and the resonant cavity position.

The gain asymmetry is one of the key figures of merit for spin lasers, and it can be viewed as crucial for their spin-selective properties, including robust spin-filtering or spin

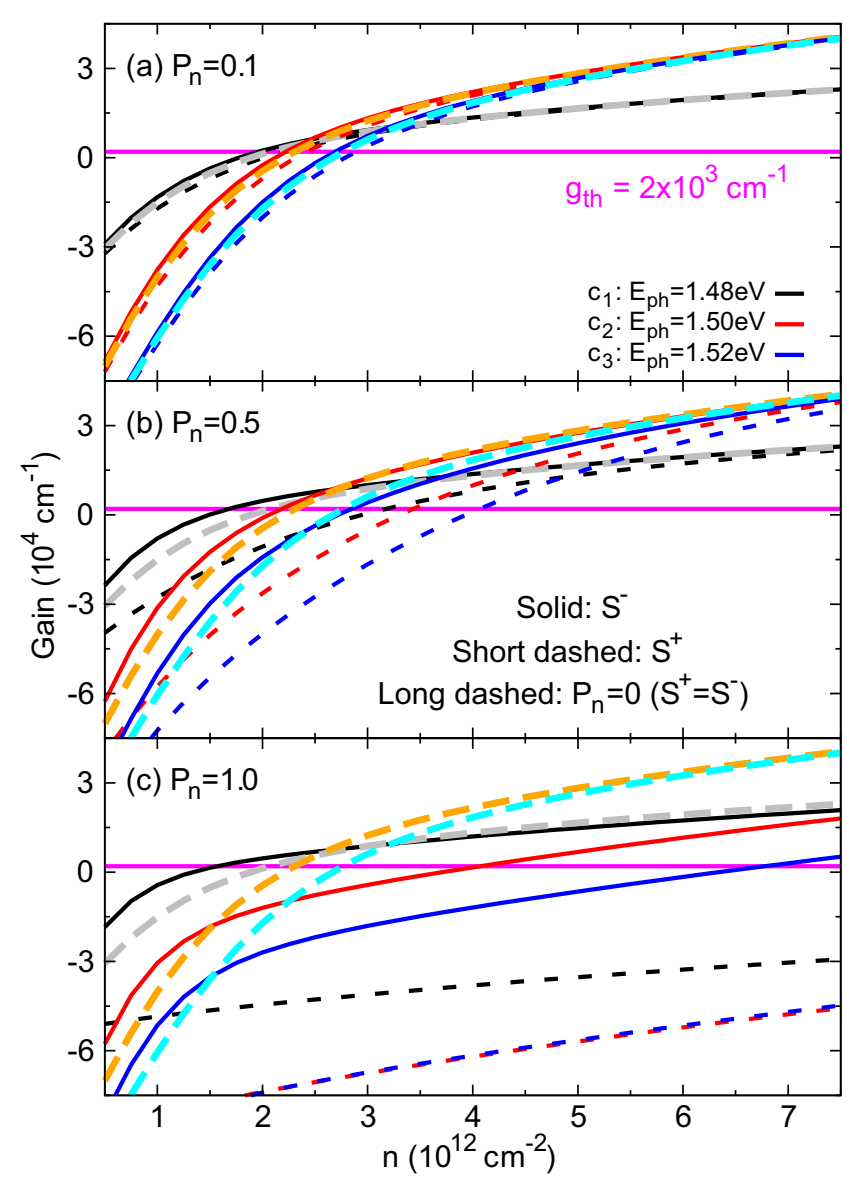

FIG. 10. (Color online) Gain as a function of carrier density for (a) $P_{n}=0.1$, (b) $P_{n}=0.5$, and (c) $P_{n}=1.0$, with the cavity choices $c_{1}, c_{2}$, and $c_{3}$. Comparing (i) solid and short-dashed lines, we can examine the spin-filtering effect, and (ii) solid and long-dashed curves, we can examine the threshold reduction. The solid horizontal line indicates the gain threshold, i.e., the losses in the cavity. To achieve the lasing, the value of gain must be greater than the gain threshold.

amplification, in which even a small $P_{n}$ (few percent) in the active region leads to an almost complete polarization of the emitted light (of just one helicity) [13]. Unfortunately, how to enhance the gain asymmetry, beyond just increasing $P_{n}$, is largely unexplored.

To establish a more systematic understanding of a gain asymmetry, we closely examine $g^{-}(\hbar \omega)-g^{+}(\hbar \omega)$ in Figs. 9(a)-9(c) for different $P_{n}$, carrier densities, and resonant cavities. Increasing $n$, the gain asymmetry peak shifts to higher $\hbar \omega$, indicating an occupation of higher-energy subbands. However, the absolute asymmetry peak is not always in the emission region. For a desirable operation of a spin laser, we should seek a large gain asymmetry with a positive (and a preferably large) gain. Complementary information is given by Figs. 9(d)-9(f) with a density evolution of $g^{-}-g^{+}$for different cavity positions and $P_{n}$. Again, we see that the gain asymmetry peak can be attained outside of the lasing region.

The results in Fig. 9 have shown a complex evolution of the gain asymmetry with the cavity position and carrier density. We now repeat a similar analysis for the gain itself in Fig. 10. 
The gain calculated for two helicities and unpolarized light $\left(S^{+}=S^{-}\right)$provides a useful guide for the threshold reduction and the spin-filtering effect, invoked in a simple bucket model from Fig. 1.

We first consider $P_{n}=0.1$, which shows a behavior with an increase in $n$ or, equivalently, an increase in injection, that could be expected from the bucket model. The threshold value of the gain (the onset of an overflowing bucket), $g_{\text {th }}$, is first reached for $S^{-}$, then for unpolarized light, a sign of threshold reduction, and finally for $S^{+}$(a subdominant helicity from the conservation of angular momentum and $P_{n}>0$ ). Therefore, there is a spin-filtering interval of $n$ (small, since $P_{n}$ itself is small) where we expect lasing with only one helicity. A similar behavior appears for all the cavity choices: $c_{1}, c_{2}$, and $c_{3}$.

We next turn to $P_{n}=0.5$, where $c_{1}$ shows trends expected both from the bucket model and early work on spin lasers [4,5]. An increase from $P_{n}=0.1$ to 0.5 enhances the threshold reduction and the spin-filtering interval. However, different cavity positions $c_{2}$ and $c_{3}$ reveal a different behavior. There is a region where unpolarized light $S^{+}=S^{-}$(long dashed lines) yields a greater gain than for $S^{-}$(solid lines). For $c_{3}$ the threshold is attained at smaller $n$ for unpolarized light than for negative helicity, i.e., there is no threshold reduction [53]. With $P_{n}=1.0$, the threshold reduction is only possible for $c_{1}$.

These results reinforce the possibility for a versatile spinVCSEL design by a careful choice of the resonant cavity, but they also caution that, depending on the given resonant cavity, the usual intuition about the influence of carrier density and spin polarization on the laser operation may not be appropriate.

\section{STRAIN-INDUCED BIREFRINGENCE}

An important implication of an anisotropic dielectric function is the phenomenon of birefringence in which the refractive index, and thus the phase velocity of light, depends on the polarization of light [34]. Due to phase anisotropies in the laser cavity [54], the emitted frequencies of linearly polarized light in the $x$ and $y$ directions $\left(S^{x}\right.$ and $\left.S^{y}\right)$ are usually different. Such birefringence is often undesired for the operation of conventional lasers since it is the origin for the typical complex polarization dynamics and chaotic polarization switching behavior in VCSELs [32,55-58]. While strong values of birefringence are usually considered to be an obstacle for the polarization control in spin-polarized lasers $[6,15]$, the combination of a spin-induced gain asymmetry with birefringence in spin-VCSELs allows us to generate fast and controllable oscillations between $S^{+}$and $S^{-}$polarizations $[12,16]$. The frequency of these polarization oscillations is determined by the linear birefringence in the VCSEL cavity, and it can be much higher than the frequency of relaxation oscillations of the carrier-photon system in conventional VCSELs. This may pave the way toward ultrahigh bandwidth operation for optical communications [12,25,59].

To investigate birefringence effects in the active region of a conventional laser, we consider uniaxial strain by extending the lattice constant in the $x$ direction. For simplicity, we assume the barrier to have the same lattice constant as GaAs, $5.6533 \AA$, in the $y$ direction. Therefore, both barrier and well regions will have the same extension in the $x$ direction. For $a_{x}=5.6544 \AA$

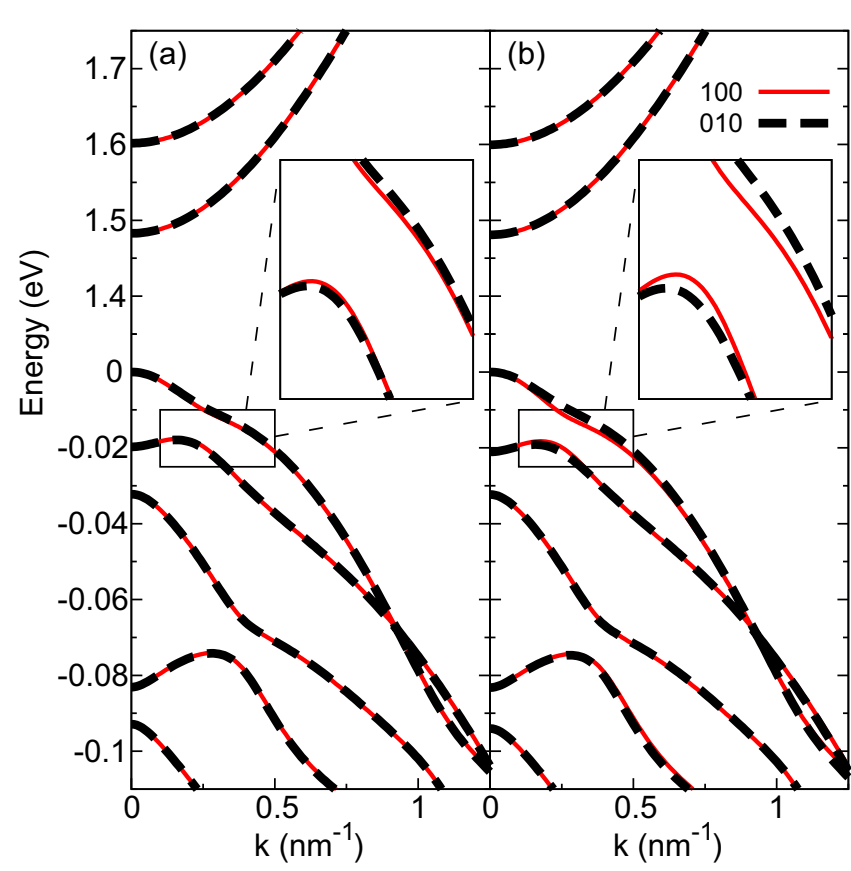

FIG. 11. (Color online) Band structure with uniaxial strain in the active region for (a) $\varepsilon_{x x} \sim 0.019 \%$ and (b) $\varepsilon_{x x} \sim 0.058 \%$. The inset shows a zoom around the HH1 and LH1 interaction region, where the difference between [100] and [010] directions is more visible. The energy gap of the system is $E_{g} \sim 1.483 \mathrm{eV}$ for case (a) and $E_{g} \sim 1.481 \mathrm{eV}$ for case (b).

we have the corresponding element of the strain tensor $\varepsilon_{x x} \sim$ $0.019 \%$, while $a_{x}=5.6566 \AA$ gives $\varepsilon_{x x} \sim 0.058 \%$.

The effect of uniaxial strain in the band structure is presented in Figs. 11(a) and 11(b) for $\varepsilon_{x x} \sim 0.019 \%$ and $\varepsilon_{x x} \sim 0.058 \%$, respectively. The labeling and ordering of subbands is the same as that in Fig. 5(a). Just this slight anisotropy in the $x$ and $y$ lattice constants creates a difference in subbands for the [100] and [010] directions. In the inset we show the region around the anticrossing of HH1 and LH1 subbands, where the difference is more visible.

In addition to the differences induced in the band structure, the uniaxial strain also induces a change in the dipole selection rules between $S^{x}$ and $S^{y}$ light polarizations, which can be seen in the gain spectra we present in Figs. 12(a) and 12(b) for $\varepsilon_{x x} \sim 0.019 \%$ and $\varepsilon_{x x} \sim 0.058 \%$, respectively. Reflecting the features of the band structure, we notice for the emission region of the gain spectra that the largest difference between $g^{x}$ and $g^{y}$ is around the HH1 and LH1 energy regions (between $c_{1}$ and $c_{3}$ cavity positions, approximately). In the absorption regime (negative gain) we notice $g^{x}<g^{y}$, while in the emission regime (positive gain) we have $g^{x}>g^{y}$. This feature is more visible in Fig. 12(b).

To calculate the birefringence coefficient in the active region, we used the definition of Ref. [60], given by

$$
\gamma_{p}(\omega)=-\frac{\omega}{2 n_{e} n_{g}} \delta \varepsilon_{r}(\omega),
$$

where $\omega$ is the frequency of the longitudinal mode in the cavity, $n_{e}$ is the effective index of refraction of the cavity, and $n_{g}$ is the group refractive index. For simplicity, we assume $n_{e}=n_{g}$. 


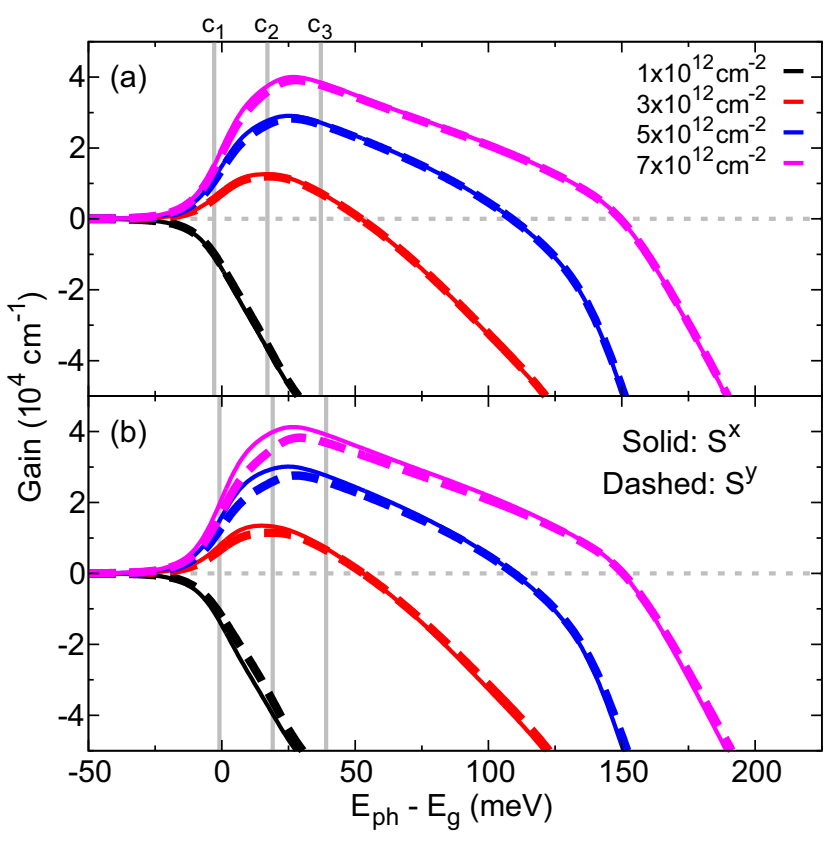

FIG. 12. (Color online) Uniaxial strain modification of gain spectra for strain (a) $\varepsilon_{x x} \sim 0.019 \%$ and (b) $\varepsilon_{x x} \sim 0.058 \%$. The anisotropy in the lattice constants for the $x$ and $y$ directions modifies the output light polarization of the laser. Since there are no spinpolarized carriers in the system, $g^{+}=g^{-}$.

The real part of the dielectric function can be obtained from the imaginary part using the Kramers-Kronig relations [42].

We present the birefringence coefficient in Figs. 13(a) and 13(b) for $\varepsilon_{x x} \sim 0.019 \%$ and $\varepsilon_{x x} \sim 0.058 \%$, respectively. We notice that this strain in the active region, responsible for modest changes in the gain spectra, produces birefringence values of the order of $10^{11}-10^{12} \mathrm{~Hz}$, which may be exploited to generate fast polarization oscillations. Furthermore, when increasing the strain amount by $\sim 0.04 \%$ from case (a) to case (b), the value of $\gamma_{p}$ increases approximately threefold [61]. We also included in our calculations spin-polarized electrons, and we notice that they have only a small influence in the birefringence coefficient. Although they change $\left|g^{x}\right|$ and $\left|g^{y}\right|$ slightly, the asymmetry is not affected at all for small spin polarizations of $100 \%$, which are relevant values in real devices.

Investigating the effect of different cavity designs, we present the values of $\gamma_{p}$ in Figs. 14(a) and 14(b) for $\varepsilon_{x x} \sim$ $0.019 \%$ and $\varepsilon_{x x} \sim 0.058 \%$, respectively. We chose the same photon energies as for the case without birefringence assuming that the different values for the strain-induced birefringence in the active region will not significantly affect the cavity resonance for reasons of simplicity. For the two different strain types, the behavior of $\gamma_{p}$ is very similar for the same resonance energy. Comparing different cavity designs, we observe that for $c_{1}$, the value of $\gamma_{p}$ strongly decreases and also changes sign with the carrier density, $n$. In contrast, for $c_{2}$ and $c_{3}, \gamma_{p}$ is always positive. After a slow increase with $n, \gamma_{p}$ becomes flat and nearly independent of the carrier density.

For consistency, we have also calculated the DBR contributions using the approach given by Mulet and Balle [60]. For large anisotropies in the DBR, the birefringence coefficient is on the order of $10^{10} \mathrm{~Hz}$, consistent with the

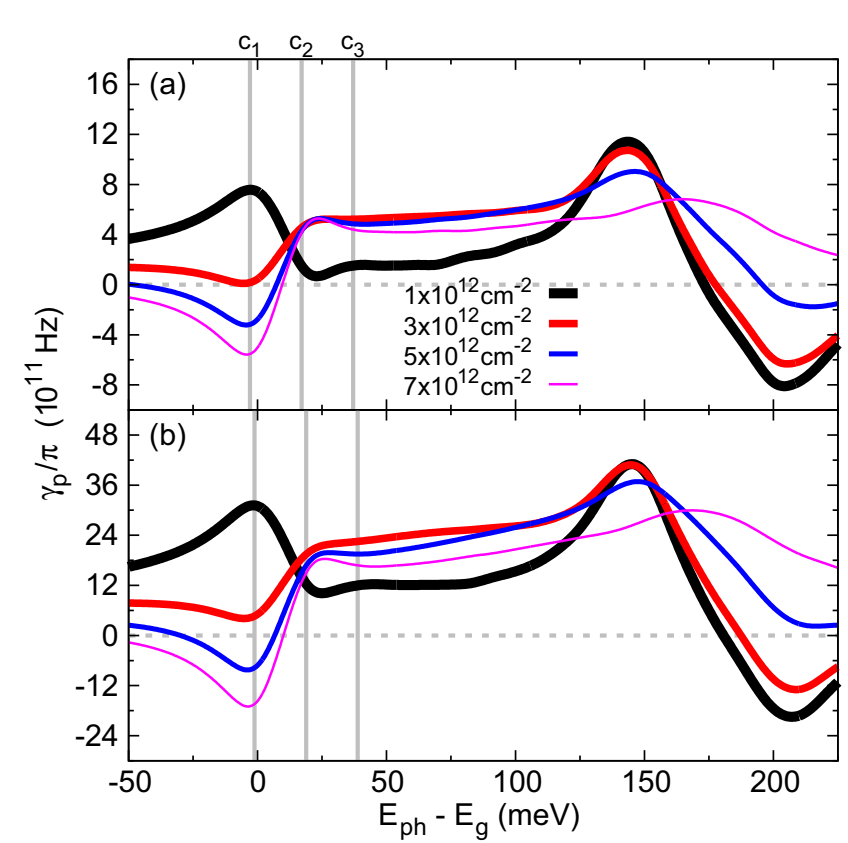

FIG. 13. (Color online) Birefringence coefficient as a function of photon energy considering (a) $\varepsilon_{x x} \sim 0.019 \%$ and (b) $\varepsilon_{x x} \sim 0.058 \%$. Just an increase of $0.0022 \AA$ in $a_{x}$ increases $\gamma_{p}$ by approximately three times. The two peaks, around $E_{\mathrm{ph}}-E_{\mathrm{g}} \sim 0 \mathrm{meV}$ and $E_{\mathrm{ph}}-E_{\mathrm{g}} \sim$ $150 \mathrm{meV}$, are related to transitions from CB1 and CB2. Transitions related to $\mathrm{CB} 2$ are in the absorption regime, not visible in Fig. 12.

measurements given by van Exter et al. [55]. Therefore, for the investigated strain conditions, the main contribution to $\gamma_{p}$ comes from the active region, and it is a very versatile parameter that can be fine-tuned using both carrier density and cavity designs, possibly even changing its sign and reaching carrier density-independent regions.

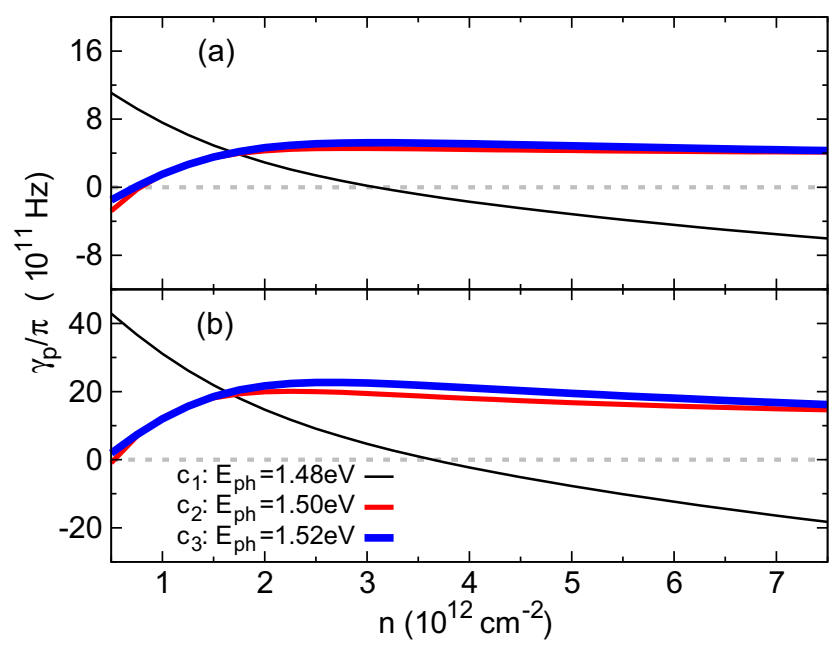

FIG. 14. (Color online) Birefringence coefficient as a function of the carrier density for (a) $\varepsilon_{x x} \sim 0.019 \%$ and (b) $\varepsilon_{x x} \sim 0.058 \%$. For different cavity designs, the behavior of $\gamma_{p}$ can be completely different. The carrier density values where $\gamma_{p}$ changes sign in cavity $c_{1}$ and the flat region in cavities $c_{2}$ and $c_{3}$ are already in the lasing regime. 


\section{ULTRAHIGH-FREQUENCY OPERATION}

Lasers could provide the next generation of parallel optical interconnects and optical information processing [34-36,62-65]. The growth in communication [66] and massive data centers [67] will pose further limitations on interconnects [68]. Conventional metallic interconnects used in multicore microprocessors are increasingly recognized as the bottleneck in maintaining Moore's law scaling and the main source of power dissipation $[65,68]$. Optical interconnects can effectively address the related limitations, such as the electromagnetic crosstalk and signal distortion, while providing a much larger bandwidth $[64,65]$. VCSELs are considered particularly suitable for short-haul communication and on-chip interconnects [36]. However, to fully utilize their potential, it would be important to explore the paths for their high-frequency operation and achieve a higher modulation bandwidth, limited for conventional lasers to about $\sim 50 \mathrm{GHz}[36,69]$.

How can we understand the frequency limitation of a laser? Why would a higher frequency modulation lead to a decrease in a signal-to-noise ratio and limit the effective bandwidth? An accurate analogy is provided by a driven and damped harmonic oscillator. The laser response, just like the harmonic oscillator, is unable to follow a high enough modulation frequency. A Lorentzian-like frequency-dependent displacement of a harmonic oscillator closely matches a modulation response of a laser, decreasing as $1 / \omega$, above the corresponding resonance frequency, known as the relaxation oscillation frequency, $\omega_{R}$, representing a natural oscillation between the carriers and photons and often used to estimate the bandwidth of a laser $[34,36,70]$.

To realize a high-speed operation in conventional lasers requires a careful design and optimization of many parameters. Attaining a high $\omega_{R}$ is closely related to optimizing the gain, which increases with $n$ [71] but decreases with photon density $S$, known as the gain compression [72], which would be desirable to minimize. For a small-signal modulation $S(t)=S_{0}+\delta S(t)$, above the threshold [34],

$$
\omega_{R}^{2} \approx v_{g}(d g / d n) S_{0} / \tau_{\mathrm{ph}},
$$

where $v_{g}$ is the group velocity of the relevant mode, $d g / d n$ is the differential gain at the threshold, and $\tau_{\mathrm{ph}}$ is the photon lifetime. While $\omega_{R}$ increases with $S_{0}$, a larger $S_{0}$, through gain compression, is detrimental by diminishing the differential gain. There are additional factors, beyond Eq. (7), required for a high $\omega_{R}$, such as minimizing the transport time for carriers to reach the active region, achieving a high carrier escape rate into the QW barriers, and minimizing extrinsic parasitic effects between the intrinsic laser and the driving circuit [36,70].

Introducing spin-polarized carriers offers additional possibilities to enhance $\omega_{R}$, corresponding to the modulation of the emitted $S$, beyond the frequencies attainable in conventional lasers. In the regime of small-signal modulation, both $\omega_{R}$ and the bandwidth have been shown to increase with an increase of the spin polarization of the injected carriers, $P_{J}$ [26,29], associated with the threshold reduction [thus for a given injection, $S_{0}$ is larger than in Eq. (7)]. Similar trends are predicted in the large-signal modulation, but the corresponding increase of $\omega_{R}$ (as compared to the conventional lasers) can exceed what would be expected based only on the threshold reduction due to $P_{J} \neq 0$ [25].
Another approach to achieve a higher $\omega_{R}$ is to use the polarization dynamics, instead of the intensity dynamics of the emitted light. The coupling between spin-polarized carriers and the light polarization in birefringent microcavities corresponds to different resonant mechanisms than those that govern the light intensity and thus to potentially higher $\omega_{R}$. Early experiments on polarization dynamics in VCSELs of Oestreich and collaborators have demonstrated spin-carrier dynamics of $120 \mathrm{GHz}$ [73]. However, their (Ga,In)As QW spin lasers operated at $10 \mathrm{~K}$ and required a large magnetic field for fast spin precession.

Could we attain similar ultrahigh frequencies at room temperature without an applied magnetic field? Our findings from Sec. VI suggest that indeed such an operation could be realized by a careful design of birefringent cavity properties providing frequency splitting of the two orthogonal linearly polarized lasing modes. While in conventional VCSEL only one linearly polarized mode is emitted, injecting spin-polarized carriers leads to circularly polarized emission and thus the operation of both linearly polarized modes at the same time. The beating between the two frequency-split linearly polarized modes creates polarization oscillations with frequency determined by the birefringence rate, $\gamma_{p} / \pi[12,16]$.

Strain-induced values of $\gamma_{p}$ in the active region shown in Figs. 13 and 14 are sufficiently high to exceed the highest available frequency operation of conventional VCSELs. A strong spectral dependence of $\gamma_{p}$, including a possible sign change, requires a careful analysis of the detuning behavior, but it also provides important opportunities for desirable operation of spin-lasers. For example, a large $\gamma_{p}$ can be achieved with a very weak dependence on the carrier density. The feasibility of a high-birefringence rate is further corroborated by the experiments using mechanical strain attaining $\gamma_{p} / \pi \sim 80$ $\mathrm{GHz}$ [74], while theoretical calculations suggest even $\gamma_{p} / \pi \sim$ $400 \mathrm{GHz}$ with asymmetric photonic crystals [75].

\section{CONCLUSIONS}

Our microscopic model of optical gain is based on a similar framework previously employed for conventional lasers $[31,34,44]$ to simply elucidate how introducing spin imbalance could enable their improved dynamical operation. In contrast to the common understanding that the birefringence is detrimental for lasers, we focus on the regime of a large strain-induced birefringence to overcome frequency limitations in conventional lasers.

With a goal to maximize the birefringence-dominated bandwidth in an experimentally realized spin laser, we can use the guidance from the analysis of both high-speed conventional lasers and the steady-state operation of spin lasers to explore potential limiting factors. Future calculations should also examine the influence of a spin-dependent gain compression, Coulomb interactions [44,76,77], an active region with multiple QWs [36], spin relaxation [20,25,78], and a careful analysis of the optimal cavity position that would combine high (differential) gain, high-gain asymmetry, and high $\gamma_{p}$.

While currently the most promising path to demonstrate our predictions for ultrahigh-frequency operation is provided by optically injected spin-polarized carriers to the existing VCSELs, there are encouraging developments for electrically injected spin-polarized carriers. A challenge is to overcome 
a relatively large separation between a ferromagnetic spin injector and an active region $(>\mu \mathrm{m})$ implying that at $300 \mathrm{~K}$ recombining carriers would have only a negligible spin polarization [79]. However, room-temperature electrical injection of spin-polarized carriers has already been realized through spin-filtering by integrating nanomagnets with the active region of a VCSEL [17]. Additional efforts focus on vertical external cavity surface-emitting lasers (VECSELs) $[14,15]$, which could enable a thin-film ferromagnet to be deposited just 100-200 nm away from the active region, sufficiently close to attain a considerable spin polarization of carriers in the active region at room temperature.

Independent progress in spintronics to store and sense information using magnets with a perpendicular anisotropy [80] and to attain fast magnetization reversal [81] could also be directly beneficial for spin lasers. Electrical spin injection usually relies on magnetic thin films with in-plane anisotropy, requiring a large applied magnetic field to achieve an out-of-plane magnetization and the projection of injected spin compatible with the carrier recombination of circularly polarized light in a VCSEL geometry (along the $z$ axis; see Fig. 4). However, a perpendicular anisotropy could provide an elegant spin injection in remanence [82-84], avoiding the technologically undesirable applied magnetic field. The progress in fast magnetization reversal could stimulate implementing all-electrical schemes for spin modulation in lasers that were shown to yield an enhanced bandwidth in lasers $[12,16,25,26,29,85,86]$.

Note added in proof. After this work was completed and submitted, our predictions for high-frequency birefringence were experimentally demonstrated in similar GaAs/AlGaAs quantum well spin VCSELs revealing values of $\sim 250 \mathrm{GHz}$ [87].

\section{ACKNOWLEDGMENTS}

We thank M. R. Hofmann and R. Michalzik for valuable discussions about the feasibility of the proposed spin-lasers and the state-of-the-art active regions in conventional lasers. We thank B. Scharf for carefully reading this manuscript. This work has been supported by CNPq (Grant No. 246549/20122), FAPESP (Grants No. 2011/19333-4, No. 2012/05618-0, and No. 2013/23393-8), NSF ECCS-1508873, NSF ECCS1102092, U.S. ONR N000141310754, NSF DMR-1124601, and the German Research Foundation (DFG) Grant "Ultrafast Spin Lasers for Modulation Frequencies in the $100 \mathrm{GHz}$ Range" GE 1231/2-1.

\section{APPENDIX A}

The versatility of the $\boldsymbol{k} \cdot \boldsymbol{p}$ method has been successfully used to obtain the gain spectra in conventional lasers $[31,34,35,42,44]$, as well as to elucidate a wealth of other phenomena, such as the spin Hall effect, topological insulators, and zitterbewegung [88-90]. Our own implementation of the $\boldsymbol{k} \cdot \boldsymbol{p}$ method in this work has been previously tested in calculating the luminescence spectra in $\delta$-doped GaAs [91], confirming experimental and theoretical electronic structure for $\mathrm{GaAs}$ QWs [92] and (Al,Ga)N/GaN superlattices [93], identifying fully spin-polarized semiconductor heterostructures, based on (Zn,Co)O [94], and exploring polytypic systems consisting of zinc-blende and wurtzite crystal phases in the same nanostructure [95,96].

Before considering confined systems, it is important to investigate the corresponding bulk crystal structure and construct the functional form of the Hamiltonian. For zinc-blende crystals, the bulk basis set that describes the lower conduction and top valence bands is [20,97-100]

$$
\begin{aligned}
|\mathrm{CB} \Uparrow\rangle & =|S \uparrow\rangle, \\
|\mathrm{CB} \Downarrow\rangle & =|S \downarrow\rangle, \\
|\mathrm{HH} \uparrow\rangle & =|(X+i Y) \uparrow\rangle / \sqrt{2}, \\
|\mathrm{LH} \Uparrow\rangle & =i|(X+i Y) \downarrow-2 Z \uparrow\rangle / \sqrt{6}, \\
|\mathrm{LH} \Downarrow\rangle & =|(X-i Y) \uparrow+2 Z \downarrow\rangle / \sqrt{6}, \\
|\mathrm{HH} \Downarrow\rangle & =i|(X-i Y) \downarrow\rangle / \sqrt{2}, \\
|\mathrm{SO} \Uparrow\rangle & =|(X+i Y) \downarrow+Z \uparrow\rangle / \sqrt{3}, \\
|\mathrm{SO} \Downarrow\rangle & =i|-(X-i Y) \uparrow+Z \downarrow\rangle / \sqrt{3},
\end{aligned}
$$

where, compared to Fig. 4(a), we also introduce the spin-orbit spin-split-off subbands $|\mathrm{SO}\rangle$. Here $|S\rangle$ and $|X\rangle,|Y\rangle,|Z\rangle$ are the basis states for irreducible representations $\Gamma_{1} \sim x^{2}+y^{2}+z^{2}$ and $\Gamma_{15} \sim x, y, z$, having an orbital angular momentum $l=0$ and 1 , respectively. The single arrows $(\uparrow, \downarrow)$ represent the projection of spin angular momentum $s=1 / 2$ on the $+z$ axis, while the double arrows $(\Uparrow, \Downarrow)$ represent the projection of total angular momentum on the $+z$ axis. Rewriting the basis set (A1) in terms of the total angular momentum $j$ and its projection $m_{j},\left|j, m_{j}\right\rangle$, we have

$$
\begin{aligned}
|\mathrm{CB} \Uparrow(\Downarrow)\rangle & =|1 / 2,1 / 2(-1 / 2)\rangle, \\
|\mathrm{HH} \Uparrow(\Downarrow)\rangle & =|3 / 2,3 / 2(-3 / 2)\rangle, \\
|\mathrm{LH} \Uparrow(\Downarrow)\rangle & =|3 / 2,1 / 2(-1 / 2)\rangle, \\
|\mathrm{SO} \Uparrow(\Downarrow)\rangle & =|1 / 2,1 / 2(-1 / 2)\rangle .
\end{aligned}
$$


In the basis set of Eq. (A1), the $\boldsymbol{k} \cdot \boldsymbol{p}$ term in Eq. (1) is

$$
H_{\boldsymbol{k} \cdot \boldsymbol{p}}=\left[\begin{array}{cccccccc}
U & 0 & i P_{+} & \sqrt{\frac{2}{3}} P_{z} & \frac{i}{\sqrt{3}} P_{-} & 0 & \frac{i}{\sqrt{3}} P_{z} & \sqrt{\frac{2}{3}} P_{-} \\
0 & U & 0 & -\frac{1}{\sqrt{3}} P_{+} & i \sqrt{\frac{2}{3}} P_{z} & -P_{-} & i \sqrt{\frac{2}{3}} P_{+} & -\frac{1}{\sqrt{3}} P_{z} \\
-i P_{-} & 0 & Q & S & R & 0 & \frac{i}{\sqrt{2}} S & -i \sqrt{2} R \\
\sqrt{\frac{2}{3}} P_{z} & -\frac{1}{\sqrt{3}} P_{-} & S^{\dagger} & T & 0 & R & -\frac{i}{\sqrt{2}}(Q-T) & i \sqrt{\frac{3}{2}} S \\
-\frac{i}{\sqrt{3}} P_{+} & -i \sqrt{\frac{2}{3}} P_{z} & R^{\dagger} & 0 & T & -S & -i \sqrt{\frac{3}{2}} S^{\dagger} & -\frac{i}{\sqrt{2}}(Q-T) \\
0 & -P_{+} & 0 & R^{\dagger} & -S^{\dagger} & Q & -i \sqrt{2} R^{\dagger} & -\frac{i}{\sqrt{2}} S^{\dagger} \\
-\frac{i}{\sqrt{3}} P_{z} & -i \sqrt{\frac{2}{3}} P_{-} & -\frac{i}{\sqrt{2}} S^{\dagger} & \frac{i}{\sqrt{2}}(Q-T) & i \sqrt{\frac{3}{2}} S & i \sqrt{2} R & \frac{1}{2}(Q+T)-\Delta_{\mathrm{SO}} & 0 \\
\sqrt{\frac{2}{3}} P_{+} & -\frac{1}{\sqrt{3}} P_{z} & i \sqrt{2} R^{\dagger} & -i \sqrt{\frac{3}{2}} S^{\dagger} & \frac{i}{\sqrt{2}}(Q-T) & \frac{i}{\sqrt{2}} S & 0 & \frac{1}{2}(Q+T)-\Delta_{\mathrm{SO}}
\end{array}\right],
$$

with elements

$$
\begin{aligned}
Q & =-k_{x}\left(\tilde{\gamma}_{1}+\tilde{\gamma}_{2}\right) k_{x}-k_{y}\left(\tilde{\gamma}_{1}+\tilde{\gamma}_{2}\right) k_{y}-k_{z}\left(\tilde{\gamma}_{1}-2 \tilde{\gamma}_{2}\right) k_{z}, \\
T & =-k_{x}\left(\tilde{\gamma}_{1}-\tilde{\gamma}_{2}\right) k_{x}-k_{y}\left(\tilde{\gamma}_{1}-\tilde{\gamma}_{2}\right) k_{y}-k_{z}\left(\tilde{\gamma}_{1}+2 \tilde{\gamma}_{2}\right) k_{z}, \\
S & =i \sqrt{3}\left[\left(k_{x} \tilde{\gamma}_{3} k_{z}+k_{z} \tilde{\gamma}_{3} k_{x}\right)-i\left(k_{y} \tilde{\gamma}_{3} k_{z}+k_{z} \tilde{\gamma}_{3} k_{y}\right)\right], \\
R & =-\sqrt{3}\left[\left(k_{x} \tilde{\gamma}_{2} k_{x}-k_{y} \tilde{\gamma}_{2} k_{y}\right)-i\left(k_{x} \tilde{\gamma}_{3} k_{y}+k_{y} \tilde{\gamma}_{3} k_{x}\right)\right], \\
U & =E_{g}+k_{x} A k_{x}+k_{y} A k_{y}+k_{z} A k_{z}, \\
P_{ \pm} & =(1 / 2 \sqrt{2})\left[P\left(k_{x} \pm i k_{y}\right)+\left(k_{x} \pm i k_{y}\right) P\right], \\
P_{z} & =(1 / 2)\left(P k_{z}+k_{z} P\right),
\end{aligned}
$$

where $\tilde{\gamma}_{1}, \tilde{\gamma}_{2}, \tilde{\gamma}_{3}$, and $A$, given in units of $\hbar^{2} / 2 m_{0}$, are the effective-mass parameters of the valence and conduction bands, respectively, explicitly given below. The gap is $E_{g}$, the spin-orbit splitting at the $\Gamma$ point is $\Delta_{\text {SO }}$, and $P$ is the Kane parameter of the interband interaction, defined as

$$
P=-i \frac{\hbar}{m_{0}}\left\langle\alpha\left|p_{l}\right| S\right\rangle
$$

with $\alpha=X, Y, Z$ and $l=x, y, z$.

The formulation of a bulk $\boldsymbol{k} \cdot \boldsymbol{p}$ model can vary significantly in its complexity, the choice of the specific system, and the number of bands included. In the description of zinc-blende structures, usually either $6 \times 6$ or $8 \times 8$ models are employed [98]. In the first case, the information of the valence and conduction band is decoupled, while in the second case their coupling is explicitly included. Their effective-mass parameters are connected by

$$
\begin{aligned}
\tilde{\gamma}_{1} & =\gamma_{1}-E_{P} / 3 E_{g}, \\
\tilde{\gamma}_{2} & =\gamma_{2}-E_{P} / 6 E_{g}, \\
\tilde{\gamma}_{3} & =\gamma_{3}-E_{P} / 6 E_{g}, \\
A & =\frac{1}{m_{e}^{*}}-\left(\frac{E_{g}+\frac{2}{3} \Delta_{\mathrm{SO}}}{E_{g}+\Delta_{\mathrm{SO}}}\right) \frac{E_{P}}{E_{g}}, \\
E_{P} & =2 m_{0} P^{2} / \hbar^{2}
\end{aligned}
$$

where $\tilde{\gamma}_{1,2,3}$ are used in the $8 \times 8$ model and $\gamma_{1,2,3}$ in the $6 \times 6$ model, which can also be related to the tight-binding parameters [92]. To recover the $6 \times 6$ model from the $8 \times 8$ model, we set $P=0$ in Eqs. (A3), (A4), and (A6).

The strain term, $H_{\mathrm{st}}$, takes a form similar to Eq. (A3) but without the $E_{g}, \Delta_{\mathrm{SO}}$, and $P$ parameters. The matrix elements can be written as

$$
\begin{aligned}
Q_{\mathrm{st}} & =-a_{v}\left(\varepsilon_{x x}+\varepsilon_{y y}+\varepsilon_{z z}\right)-\frac{b}{2}\left(\varepsilon_{x x}+\varepsilon_{y y}-2 \varepsilon_{z z}\right), \\
T_{\mathrm{st}} & =-a_{v}\left(\varepsilon_{x x}+\varepsilon_{y y}+\varepsilon_{z z}\right)+\frac{b}{2}\left(\varepsilon_{x x}+\varepsilon_{y y}-2 \varepsilon_{z z}\right), \\
S_{\mathrm{st}} & =d\left(\varepsilon_{y z}+i \varepsilon_{x z}\right), \\
R_{\mathrm{st}} & =-\frac{\sqrt{3} b}{2}\left(\varepsilon_{x x}-\varepsilon_{y y}\right)+i d \varepsilon_{x y}, \\
U_{\mathrm{st}} & =a_{c}\left(\varepsilon_{x x}+\varepsilon_{y y}+\varepsilon_{z z}\right),
\end{aligned}
$$

with $a_{v}, b$, and $d$ representing the deformation potentials for the valence band and $a_{c}$ for the conduction band. The strain tensor components are given by $\varepsilon_{i j}(i, j=x, y, z)$.

To treat a QW system, which now lacks translational symmetry along the growth direction, we can replace the exponential part of Bloch's theorem by a generic function. This procedure is called the envelope-function approximation [98], and it leads to the dependence along the growth direction of the $\boldsymbol{k} \cdot \boldsymbol{p}$ and strain parameters in Hamiltonian terms $H_{\boldsymbol{k} \cdot \boldsymbol{p}}(z)$ and $H_{\mathrm{st}}(z)$. Also, the band-offset at the interface of different materials is taken into account in the term $H_{\mathrm{O}}(z)$,

$$
H_{\mathrm{O}}(z)=\operatorname{diag}\left[\delta_{V}(z), \ldots, \delta_{V}(z), \delta_{C}(z), \delta_{C}(z)\right],
$$

where $\delta_{V(C)}(z)$ describes the energy change in the valence (conduction) band.

Under the envelope-function approximation, the QW Hamiltonian from Eq. (1) is now described by a system of eight coupled differential equations that do not generally have analytical solutions. We solve these equations numerically using the plane-wave expansion for the $z$-dependent parameters and envelope functions. Details of the envelope-function approximation and plane-wave expansion for QW systems can be found in Refs. [95,96,99].

\section{APPENDIX B}

The interband dipole transition amplitude that appears in Eq. (3) is given by

$$
p_{c v \vec{k}}^{a}=\langle c, \vec{k}|\hat{a} \cdot \vec{p}| v, \vec{k}\rangle,
$$


and for the light polarization $S^{ \pm}$we have

$$
\hat{a}=\frac{1}{\sqrt{2}}(\hat{x} \pm i \hat{y})
$$

and therefore

$$
\hat{a} \cdot \vec{p}=\frac{p_{x} \pm i p_{y}}{\sqrt{2}} .
$$

In the simplified QW of Fig. 4, we are showing the selection rules for $\vec{k}=0$ and assuming the conduction band as $|c, 0\rangle=$ $|\mathrm{CB} \Uparrow(\Downarrow)\rangle$ and the valence band as $|v, 0\rangle=|\mathrm{HH} \Uparrow(\Downarrow)\rangle$ or $|v, 0\rangle=|\mathrm{LH} \Uparrow(\Downarrow)\rangle$. Calculating the matrix elements between these states, we obtain

$$
\left\langle\mathrm{CB} \Uparrow\left|p_{ \pm}\right| \mathrm{HH} \Uparrow\right\rangle=\left\langle S \uparrow\left|\frac{p_{x} \pm i p_{y}}{\sqrt{2}}\right| \frac{1}{\sqrt{2}}(X+i Y) \uparrow\right\rangle=\frac{1}{2}\left\langle S \uparrow\left|p_{x}\right| X \uparrow\right\rangle \mp \frac{1}{2}\left\langle S \uparrow\left|p_{y}\right| Y \uparrow\right\rangle,
$$

which is nonzero only for $p_{-}$,

$$
\left\langle\mathrm{CB} \Downarrow\left|p_{ \pm}\right| \mathrm{HH} \Downarrow\right\rangle=\left\langle S \downarrow\left|\frac{p_{x} \pm i p_{y}}{\sqrt{2}}\right| \frac{i}{\sqrt{2}}(X-i Y) \downarrow\right\rangle=\frac{i}{2}\left\langle S \downarrow\left|p_{x}\right| X \downarrow\right\rangle \pm \frac{i}{2}\left\langle S \downarrow\left|p_{y}\right| Y \downarrow\right\rangle,
$$

which is nonzero only for $p_{+}$,

$$
\left\langle\mathrm{CB} \Uparrow\left|p_{ \pm}\right| \mathrm{LH} \Downarrow\right\rangle=\left\langle S \uparrow\left|\frac{p_{x} \pm i p_{y}}{\sqrt{2}}\right| \frac{1}{\sqrt{6}}[(X-i Y) \uparrow+2 Z \downarrow]\right\rangle=\frac{1}{2 \sqrt{3}}\left\langle S \uparrow\left|p_{x}\right| X \uparrow\right\rangle \pm \frac{1}{2 \sqrt{3}}\left\langle S \uparrow\left|p_{y}\right| Y \uparrow\right\rangle,
$$

which is nonzero only for $p_{+}$, and

$$
\left\langle\mathrm{CB} \Downarrow\left|p_{ \pm}\right| \mathrm{LH} \Uparrow\right\rangle=\left\langle S \downarrow\left|\frac{p_{x} \pm i p_{y}}{\sqrt{2}}\right| \frac{i}{\sqrt{6}}[(X+i Y) \downarrow-2 Z \uparrow]\right\rangle=\frac{1}{2 \sqrt{3}}\left\langle S \downarrow\left|p_{x}\right| X \downarrow\right\rangle \mp \frac{1}{2 \sqrt{3}}\left\langle S \downarrow\left|p_{y}\right| Y \downarrow\right\rangle,
$$

which is nonzero only for $p_{-}$.

In addition to Eqs. (B4)-(B7), we can conclude that $\left\langle\mathrm{CB} \Uparrow\left|p_{ \pm}\right| \mathrm{HH} \Downarrow\right\rangle=\left\langle\mathrm{CB} \Downarrow\left|p_{ \pm}\right| \mathrm{HH} \Uparrow\right\rangle=0$ and $\left\langle\mathrm{CB} \Uparrow\left|p_{ \pm}\right| \mathrm{LH} \Uparrow\right\rangle=$ $\left\langle\mathrm{CB} \Downarrow\left|p_{ \pm}\right| \mathrm{LH} \Downarrow\right\rangle=0$, independent of the light polarization.

[1] Optical Orientation, edited by F. Meier and B. P. Zakharchenya (North-Holland, New York, 1984).

[2] S. Hallstein, J. D. Berger, M. Hilpert, H. C. Schneider, W. W. Rühle, F. Jahnke, S. W. Koch, H. M. Gibbs, G. Khitrova, and M. Oestreich, Phys. Rev. B 56, R7076 (1997).

[3] H. Ando, T. Sogawa, and H. Gotoh, Appl. Phys. Lett. 73, 566 (1998).

[4] J. Rudolph, D. Hägele, H. M. Gibbs, G. Khitrova, and M. Oestreich, Appl. Phys. Lett. 82, 4516 (2003); J. Rudolph, S. Döhrmann, D. Hägele, M. Oestreich, and W. Stolz, ibid. 87, 241117 (2005).

[5] M. Holub, J. Shin, D. Saha, and P. Bhattacharya, Phys. Rev. Lett. 98, 146603 (2007).

[6] S. Hövel, A. Bischoff, N. C. Gerhardt, M. R. Hofmann, T. Ackemann, A. Kroner, and R. Michalzik, Appl. Phys. Lett. 92, 041118 (2008).

[7] D. Basu, D. Saha, C. C. Wu, M. Holub, Z. Mi, and P. Bhattacharya, Appl. Phys. Lett. 92, 091119 (2008).

[8] D. Basu, D. Saha, and P. Bhattacharya, Phys. Rev. Lett. 102, 093904 (2009).

[9] D. Saha, D. Basu, and P. Bhattacharya, Phys. Rev. B 82, 205309 (2010).

[10] H. Fujino, S. Koh, S. Iba, T. Fujimoto, and H. Kawaguchi, Appl. Phys. Lett. 94, 131108 (2009).
[11] M. Li, H. Jähme, H. Soldat, N. C. Gerhardt, M. R. Hofmann, T. Ackemann, A. Kroner, and R. Michalzik, Appl. Phys. Lett. 97, 191114 (2010).

[12] N. C. Gerhardt, M. Y. Li, H. Jähme, H. Höpfner, T. Ackemann, and M. R. Hofmann, Appl. Phys. Lett. 99, 151107 (2011).

[13] S. Iba, S. Koh, K. Ikeda, and H. Kawaguchi, Appl. Phys. Lett. 98, 081113 (2011).

[14] J. Frougier, G. Baili, M. Alouini, I. Sagnes, H. Jaffrès, A. Garnache, C. Deranlot, D. Dolfi, and J.-M. George, Appl. Phys. Lett. 103, 252402 (2013).

[15] J. Frougier, G. Baili, I. Sagnes, D. Dolfi, J.-M. George, and M. Alouini, Opt. Express 23, 9573 (2015).

[16] H. Höpfner, M. Lindemann, N. C. Gerhardt, and M. R. Hofmann, Appl. Phys. Lett. 104, 022409 (2014).

[17] J.-Y. Cheng, T.-M. Wond, C.-W. Chang, C.-Y. Dong, and Y.-F. Chen, Nat. Nanotech. 9, 845 (2014).

[18] S. S. Alharthi, A. Hurtado, V.-M. Korpijarvi, M. Guina, I. D. Henning, and M. J. Adams, Appl. Phys. Lett. 106, 021117 (2015).

[19] F.-k. Hsu, W. Xie, Y.-S. Lee, S.-D. Lin, and C.-W. Lai, Phys. Rev. B 91, 195312 (2015).

[20] I. Žutić, J. Fabian, and S. Das Sarma, Rev. Mod. Phys. 76, 323 (2004). 
[21] J. Fabian, A. Mathos-Abiague, C. Ertler, P. Stano, and I. Žutić, Acta Phys. Slovaca 57, 565 (2007).

[22] Handbook of Spin Transport and Magnetism, edited by E. Y. Tsymbal and I. Žutić (Chapman and Hall/CRC, New York, 2011).

[23] Spin Dependent Transport in Magnetic Nanostructures, edited by S. Maekawa, and T. Shinjo (Taylor \& Francis, New York, 2002).

[24] S. Das Sarma, J. Fabian, X. D. Hu, and I. Žutić, Superlatt. Microstruct. 27, 289 (2000).

[25] J. Lee, S. Bearden, E. Wasner, and I. Žutić, Appl. Phys. Lett. 105, 042411 (2014).

[26] J. Lee, R. Oszwałdowski, C. Gøthgen, and I. Žutić, Phys. Rev. B 85, 045314 (2012).

[27] I. Žutić and P. E. Faria Junior, Nat. Nanotech. 9, 750 (2014).

[28] C. Gøthgen, R. Oszwałdowski, A. Petrou, and I. Žutić, Appl. Phys. Lett. 93, 042513 (2008).

[29] J. Lee, W. Falls, R. Oszwałdowski, and I. Žutić, Appl. Phys. Lett. 97, 041116 (2010).

[30] G. Boéris, J. Lee, K. Výborný, and I. Žutić, Appl. Phys. Lett. 100, 121111 (2012).

[31] S. L. Chuang, Physics of Optoelectronic Devices, 2nd ed. (Wiley, New York, 2009).

[32] M. San Miguel, Q. Feng, and J. V. Moloney, Phys. Rev. A 52, 1728 (1995).

[33] A. Gahl, S. Balle, and M. Miguel, IEEE J. Quantum Electron. 35, 342 (1999).

[34] L. A. Coldren, S. W. Corzine, and M. L. Mašović, Diode Lasers and Photonic Integrated Circuits, 2nd ed. (Wiley, Hoboken, NJ, 2012).

[35] S. F. Yu, Analysis and Design of Vertical Cavity Surface Emitting Lasers (Wiley, New York, 2003).

[36] VCSELs Fundamentals, Technology and Applications of Vertical-Cavity Surface-Emitting Lasers, edited by R. Michalzik (Springer, Berlin, 2013).

[37] R. Oszwałdowski, C. Gøthgen, and I. Žutić, Phys. Rev. B 82, 085316 (2010).

[38] M. J. Adams and D. Alexandropoulos, IEEE Photon. J. 4, 1124 (2012).

[39] Spin injection in QDs can even lead to a phonon laser. See, e.g., A. Khaetskii, V. N. Golovach, X. Hu, and I. Žutić, Phys. Rev. Lett. 111, 186601 (2013).

[40] In a simple picture, neglecting any losses, $d S(z) / d Z=g S(z)$, where $z$ is the coordinate in a small segment of the gain region [34].

[41] Gain calculations for spin-lasers were also performed using the $6 \times 6 \boldsymbol{k} \cdot \boldsymbol{p}$ method, which considers the decoupled electronic structure of the conduction and valence bands by M. Holub and B. T. Jonker, Phys. Rev. B 83, 125309 (2011). The focus was on the steady-state performance of a laser, rather than on the high-frequency operation we consider here.

[42] H. Haug and S. W. Koch, Quantum Theory of Optical and Electronic Properties of Semiconductors, 4th ed. (World Scientific, Singapore, 2004).

[43] This gain coefficient corresponds to the ratio of the number of photons emitted per second per unit volume and the number of injected photons per second per unit area, therefore having a dimension of $1 /$ length.
[44] W. W. Chow and S. W. Koch, Semiconductor-Laser Fundamentals: Physics of the Gain Materials, (Springer, New York, 1999).

[45] Within the rate-equation description, including a widely used spin-flip model, the $n_{+} p_{+}$recombination only gives $S^{-}$helicity of the emitted light. From the consideration, this means that there is only one type of hole within a four-band model (CB and VB with a twofold spin degeneracy).

[46] Analogous expressions can be introduced for the spin polarization of injection and polarization of photon density.

[47] All bulk, strain, and band-offset parameters were extracted from I. Vurgaftman, J. R. Meyer, and L. R. Ram-Mohan, J. Appl. Phys. 89, 5815 (2001).

[48] For GaAs, $m_{e}^{*}=0.067, m_{\mathrm{hh}}^{*}=0.350, m_{\mathrm{lh}}^{*}=0.090$, and $m_{\mathrm{so}}^{*}=$ 0.172 .

[49] We assume that there is no intrinsic magnetic character of the laser due to an applied magnetic field or the presence of a magnetic region.

[50] A. T. Hanbicki, B. T. Jonker, G. Itskos, G. Kioseoglou, and A. Petrou, Appl. Phys. Lett. 80, 1240 (2002).

[51] T. J. Zega, A. T. Hanbicki, S. C. Erwin, I. Žutić, G. Kioseoglou, C. H. Li, B. T. Jonker, and R. M. Stroud, Phys. Rev. Lett. 96, 196101 (2006).

[52] G. Salis, R. Wang, X. Jiang, R. M. Shelby, S. S. P. Parkin, S. R. Bank, and J. S. Harris, Appl. Phys. Lett. 87, 262503 (2005).

[53] The possibility for an increase in threshold in spin lasers with $P_{n}=1$ has been predicted in Ref. [41]. However, we show that such an increase is not universal and depends on the cavity choice, i.e., the detuning between the cavity mode and the gain peak.

[54] A simple phase condition for the standing-wave pattern [recall Fig. 2(a)] can be written as $L=m \lambda /(2 n)$ [36], where $L$ is the cavity length, $m$ is the mode index, and $\lambda$ is the wavelength of the emitted light. The polarization dependence of the refractive index, $n$, thus leads to the polarization dependence of the emitted frequency.

[55] M. P. van Exter, A. K. Jansen van Doorn, and J. P. Woerdman, Phys. Rev. A 56, 845 (1997).

[56] M. Sondermann, M. Weinkath, and T. Ackemann, IEEE J. Quantum Electron. 40, 97 (2004).

[57] M. Virte, K. Panajotov, H. Thienpont, and M. Sciamanna, Nat. Photon. 7, 60 (2012).

[58] R. Al-Seyab, D. Alexandropoulos, I. D. Henning, and M. J. Adams, IEEE Photon. J. 3, 799 (2011).

[59] N. C. Gerhardt and M. R. Hofmann, Adv. Opt. Technol. 2012, 268949 (2012).

[60] J. Mulet and S. Balle, IEEE J. Quantum Electron. 38, 291 (2002).

[61] The birefringence coefficient in a VCSEL can also be enhanced by local heating. A. K. Jansen van Doorn, M. P. van Exter, and J. P. Woerdman, IEEE J. Quantum Electron. 34, 700 (1998).

[62] Optical Fiber Telecommunications VIA Components and Subsystems, 6th ed., edited by I. P. Kaminow, T. Li, and A. E. Willner (Academic, New York, 2013).

[63] G. P. Agrawal, Fiber-Optic Communication Systems, 3rd ed. (Wiley, New York, 2002).

[64] B. Ciftcioglu, R. Berman, S. Wang, J. Hu, I. Savidis, M. Jain, D. Moore, M. Huang, E. G. Friedman, G. Wicks, and H. Wu, Opt. Express 20, 4331 (2012). 
[65] D. A. B. Miller, Proc. IEEE 97, 1166 (2009).

[66] M. Hilbert and P. López, Science 332, 60 (2011).

[67] Scalable, energy-efficient data centers and clouds, 2012, Santa Barbara, CA, http://iee.ucsb.edu/files/Institute for Energy Efficiency Data Center Report.pdf.

[68] www.itrs.net/Links/2011ITRS/2011Chapters/2011Interconnect.pdf.

[69] P. Westbergh, E. P. Haglund, E. Haglund, R. Safaisini, J. S. Gustavsson, and A. Larsson, Electron. Lett. 49, 1021 (2013).

[70] Semiconductor Lasers I, edited by E. Kapon (Academic, San Diego, 1999).

[71] We assume a typical description of an active region that is usually undoped and has a charge neutrality, $n=p$.

[72] Attributed to the spectral hole burning and carrier heating effects in Ref. [70].

[73] M. Oestreich, J. Hübner, D. Hägele, M. Bender, N. Gerhardt, M. Hofmann, W. W. Rühle, H. Kalt, T. Hartmann, P. Klar, W. Heimbrodt, and W. Stolz, Spintronics: Spin Electronics and Optoelectronics in Semiconductors, in Advances in Solid State Physics Vol. 41, edited by B. Kramer (Springer, Berlin, 2001), pp. 173-186.

[74] K. Panajotov, B. Nagler, G. Verschaffelt, A. Georgievski, H. Thienpont, J. Danckaert, and I. Veretennicoff, Appl. Phys. Lett. 77, 1590 (2000).

[75] M. Dems, T. Czyszanowski, H. Thienpont, and K. Panajotov, Opt. Commun. 281, 3149 (2008).

[76] D. Burak, J. V. Moloney, and R. Binder, Phys. Rev. A 61, 053809 (2000).

[77] G. D. Sanders, C.-K. Sun, B. Golubovic, J. G. Fujimoto, and C. J. Stanton, Phys. Rev. B 54, 8005 (1996).

[78] I. Žutić, J. Fabian, and S. Das Sarma, Appl. Phys. Lett. 82, 22 (2003).

[79] H. Soldat, M. Y. Li, N. C. Gerhardt, M. R. Hofmann, A. Ludwig, A. Ebbing, D. Reuter, A. D. Wieck, F. Stromberg, W. Keune, and H. Wende, Appl. Phys. Lett. 99, 051102 (2011).

[80] Commercial magnetic hard drives already employ ferromagnets with perpendicular anisotropy, which enables a higher information density to be stored.

[81] S. Garzon, L. Ye, R. A. Webb, T. M. Crawford, M. Covington, and S. Kaka, Phys. Rev. B 78, 180401(R) (2008).

[82] A. Sinsarp, T. Manago, F. Takano, and H. Akinaga, Jpn. J. Appl. Phys. 46, L4 (2007).
[83] S. Hövel, N. C. Gerhardt, M. R. Hofmann, F.-Y. Lo, A. Ludwig, D. Reuter, A. D. Wieck, E. Schuster, H. Wende, W. Keune, O. Petracic, and K. Westerholt, Appl. Phys. Lett. 93, 021117 (2008).

[84] J. Zarpellon, H. Jaffres, J. Frougier, C. Deranlot, J. M. George, D. H. Mosca, A. Lemaitre, F. Freimuth, Q. H. Duong, P. Renucci, and X. Marie, Phys. Rev. B 86, 205314 (2012).

[85] D. Banerjee, R. Adari, M. Murthy, P. Suggisetti, S. Ganguly, and D. Saha, J. Appl. Phys. 109, 07C317 (2011).

[86] There is also an encouraging progress in light-emitting diodes showing electrical helicity switching. N. Nishizawa, K. Nishibayashi, and H. Munekata, Appl. Phys. Lett. 104, 111102 (2014).

[87] T. Pusch, M. Lindemann, N. C. Gerhardt, M. R. Hofmann, and R. Michalzik, Electron. Lett. (to be published).

[88] S. Murakami, N. Nagaosa, and S.-C. Zhang, Science 301, 1348 (2003).

[89] M. Z. Hasan and C. L. Kane, Rev. Mod. Phys. 82, 3045 (2010).

[90] E. Bernardes, J. Schliemann, M. Lee, J. C. Egues, and D. Loss, Phys. Rev. Lett. 99, 076603 (2007).

[91] G. M. Sipahi, R. Enderlein, L. M. R. Scolfaro, J. R. Leite, E. C. F. da Silva, and A. Levine, Phys. Rev. B 57, 9168 (1998).

[92] J. Lee, K. Výborný, J. E. Han, and I. Žutić, Phys. Rev. B 89, 045315 (2014).

[93] S. C. P. Rodrigues, L. M. R. Scolfaro, J. R. Leite, and G. M. Sipahi, Appl. Phys. Lett. 76, 1015 (2000).

[94] I. S. P. Marin, G. M. Sipahi, M. A. Boselli, and I. C. da Cunha Lima, Appl. Phys. Lett. 89, 192101 (2006).

[95] P. E. Faria Junior and G. M. Sipahi, J. Appl. Phys. 112, 103716 (2012).

[96] P. E. Faria Junior, T. Campos, and G. M. Sipahi, J. Appl. Phys. 116, 193501 (2014).

[97] R. Enderlein and N. J. Horing, Fundamentals of Semiconductor Physics and Devices, 1st ed. (World Scientific, Singapore 1997).

[98] R. Winkler, Spin-orbit Coupling Effects in Two-Dimensional Electron and Hole Systems (Springer, New York, 2003).

[99] G. M. Sipahi, R. Enderlein, L. M. R. Scolfaro, and J. R. Leite, Phys. Rev. B 53, 9930 (1996).

[100] R. Enderlein, G. M. Sipahi, L. M. R. Scolfaro, and J. R. Leite, Phys. Rev. Lett. 79, 3712 (1997). 


\title{
4.2 Band structure calculations of InP wurtzite/zinc-blende quantum wells
}

\author{
$\underline{\text { P. E. Faria Junior }}$
}

Instituto de Física de São Carlos, Universidade de São Paulo, 13566-590 São Carlos, São Paulo, Brazil

\author{
G. M. Sipahi
}

Instituto de Física de São Carlos, Universidade de São Paulo, 13566-590 São Carlos, São Paulo, Brazil

Department of Physics, State University of New York at Buffalo, Buffalo, New York 14260, USA 


\title{
Band structure calculations of InP wurtzite/zinc-blende quantum wells
}

\author{
P. E. Faria Junior ${ }^{1}$ and G. M. Sipahi ${ }^{1,2}$ \\ ${ }^{1}$ Instituto de Física de São Carlos, Universidade de São Paulo, São Carlos 13560-970, SP, Brazil \\ ${ }^{2}$ Department of Physics, State University of New York at Buffalo, Buffalo, New York 14260, USA
}

(Received 6 July 2012; accepted 31 October 2012; published online 28 November 2012)

\begin{abstract}
Semiconductor nanowhiskers (NWs) made of III-V compounds exhibit great potential for technological applications. Controlling the growth conditions, such as temperature and diameter, it is possible to alternate between zinc-blende (ZB) and wurtzite (WZ) crystalline phases, giving origin to the so called polytypism. This effect has great influence in the electronic and optical properties of the system, generating new forms of confinement to the carriers. A theoretical model capable to accurately describe electronic and optical properties in these polytypical nanostructures can be used to study and develop new kinds of nanodevices. In this study, we present the development of a wurtzite/zinc-blende polytypical model to calculate the electronic band structure of nanowhiskers based on group theory concepts and the $\mathrm{k} \cdot \mathrm{p}$ method. Although the interest is in polytypical superlattices, the proposed model was applied to a single quantum well of InP to study the physics of the wurtzite/zinc-blende polytypism. By the analysis of our results, some trends can be predicted: spatial carriers' separation, predominance of perpendicular polarization (xy plane) in the luminescence spectra, and interband transition blueshifts with strain. Also, a possible range of values for the wurtzite InP spontaneous polarization is suggested. (C) 2012 American Institute of Physics. [http://dx.doi.org/10.1063/1.4767511]
\end{abstract}

\section{INTRODUCTION}

Low dimensional semiconductor structures exhibit different characteristics, ruled by the size and morphology of the system. Recently, there is an increasing interest in nanowhiskers (NWs), also known as nanowires. These are nearly one dimensional nanostructures grown perpendicular to the surface of the substrate, usually by the vapor-liquid-crystal (VLC) method. The technological applications of NWs are present in a large variety of fields: biological and chemical nanosensors, ${ }^{1-3}$ light emission devices, ${ }^{4-6}$ and field effect transistors, ${ }^{7-10}$ for example.

The first register in the literature of whiskers was made by Wagner and Ellis ${ }^{11}$ in 1964. In this classic study, the vertical growth of a Si whisker in the [111] direction, activated by droplets of $\mathrm{Au}$, using the VLC method was demonstrated. The radius of the structure is approximately the same as the catalyst droplet of Au and the vertical size depends on the ratio and time of the compound's deposition on the substrate. Although the VLC method is the most common, other methods like vapor-phase epitaxy (VPE), molecular-beam epitaxy (MBE), and magnetron deposition (MD) are also applied for the NWs growth.

In III-V compound NWs (e.g., arsenides and phosphides), a surprising characteristic is the predominance of the wurtzite (WZ) phase in the structure. Exception made for the nitrides, the stable crystalline structure of III-V compounds in the bulk form is zinc-blende ( $\mathrm{ZB})$. Although the difference between formation energy of the two phases are small, approximately $20 \mathrm{meV}$ per pair of atoms at zero pressure, high pressures would be necessary to obtain the WZ phase in the bulk form. However, reducing the dimensions of the system to the nanoscale level, such as in these NWs, the
WZ phase becomes more stable. This stability is due to the smaller surface energy of lateral faces compared to the cubic crystal. An extensive summary of NWs growth, properties, and applications was made by Dubrovskii et al. ${ }^{12}$

Controlling the growth conditions, such as temperature and diameter of the NW, it is possible to create different regions composed of $\mathrm{ZB}$ and WZ structures. ${ }^{13-20}$ The mixture of both crystalline phases in the same nanostructure is called polytypism. Such characteristic directly affects the electronic and optical properties of NWs. The detailed study of polytypism in III-V semiconductor NWs is fundamental to the development of novel functional nanodevices with a variety of features.

The theoretical tool to calculate the electronic band structure of polytypical NWs used in this paper is the $k \cdot p$ method. Although the formulation of this method has already been done for $\mathrm{ZB}$ and $\mathrm{WZ}$ crystal structures in the bulk form $^{21-25}$ and in superlattices and heterostructures, ${ }^{26-30}$ it was never applied to a polytypical case.

In order to extend the $\mathrm{k} \cdot \mathrm{p}$ method to treat WZ/ZB polytypism, we analyzed the irreducible representations (IRs) in the $\Gamma$-point of both crystal structure. Relying on the symmetry connection of the polytypical interface presented in the paper of Murayama e Nakayama, ${ }^{31}$ it was possible to describe the top valence bands and lower conduction bands of both crystal structures in the same Hamiltonian matrix. The envelope function scheme was then applied to obtain the variation of the parameters along the growth direction, coupling the different phase regions in a NW, thus completing the polytypical model. ${ }^{32}$ The effects of strain, spontaneous polarization, and piezoelectric polarization were also included in the model in order to describe a more physical system. 
The present paper is divided as follows: In Sec. II, we discuss the symmetry of $\mathrm{ZB}$ and $\mathrm{WZ}$ crystal structures and analyze how the IRs of the energy bands are connected in the polytypical interface. Section III describes the Hamiltonian terms for the polytypical model. The results, and their discussion, of InP WZ/ZB/WZ single well are found in Sec. IV and finally, in Sec. V, we draw our conclusions.

\section{SYMMETRY ANALYSIS}

\section{A. Zinc-blende and wurtzite structures}

Our formulation relies on group theory concepts and, therefore, it is necessary to understand the symmetry of the two crystal structures considered in the polytypical NWs. A good description of the concepts of space group symmetry can be found in the book of M. S. Dresselhaus, G. Dresselhaus and A. Jorio. ${ }^{33}$

The ZB structure belongs to the class of symmorphic space groups and has the $T_{d}$ symmetry as its factor group. The number of atoms in the primitive unit cell is two. Unlike $\mathrm{ZB}$, the WZ structure belongs to the class of nonsymmorphic space groups, and its factor group is isomorphic to $C_{6 v}$. The classes of symmetry operations $C_{2}, C_{6}$, and $\sigma_{v}$ are followed by a translation of $c / 2$ in the [0001] direction. WZ has four atoms in the primitive unit cell. Comparing the factor groups, one can notice that $C_{6 v}$ is less symmetric than $T_{d}$. The symmetry of the crystal structure reflects directly the number of interactions within the $\mathrm{k} \cdot \mathrm{p}$ framework: less symmetry implicates in more parameters to describe the electronic band structure coupling in the Hamiltonian.

In polytypical NWs, the common growth direction is the ZB [111], which exhibit a noticeable similarity to WZ [0001]. Actually, analyzing both crystal structures in these directions, one can describe then as stacked hexagonal layers. The $\mathrm{ZB}$ has three layers in the stacking sequence $(\mathrm{ABCABC})$ while $\mathrm{WZ}$ has only two $(\mathrm{ABAB})$, as shown in Fig. 1. The crystal structure alternation occurs when a stacking fault happens in WZ, leading to a single ZB segment, or when two twin planes appear in $\mathrm{ZB}$, originating a single $\mathrm{WZ}$ segment. $^{15}$
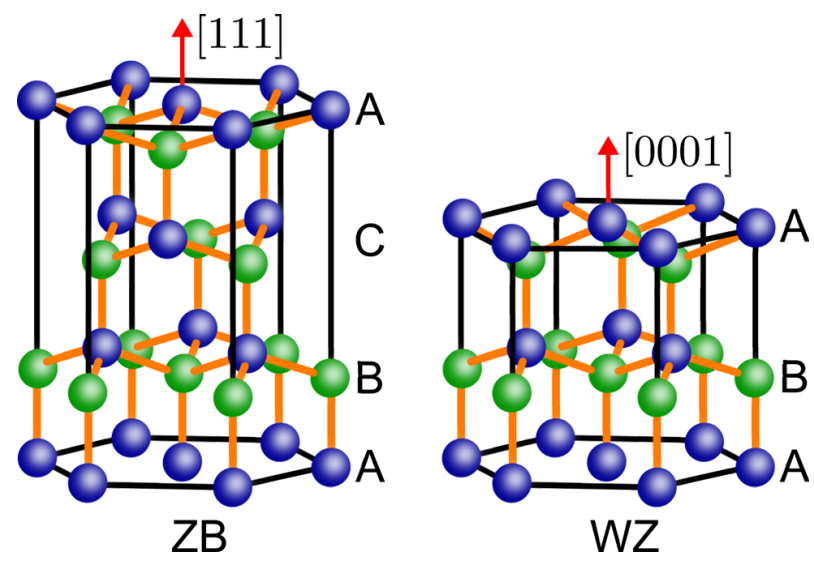

FIG. 1. ZB (left) and WZ (right) structures and their stacking sequence. The $\mathrm{ZB}$ is presented in the [111] direction. In this direction, the unit cell is twice as large as the usual ZB unit cell. ${ }^{31}$ The WZ is presented in the [0001] direction. The arrows (red lines) are the growth directions in NWs.

\section{B. Irreducible representations at the polytypical interface}

In order to correctly describe a polytypical system, it is essential to determine how the symmetry of states in both phases are connected. We used the scheme proposed by Murayama and Nakayama ${ }^{31}$ of single group IRs at the WZ/ZB interface. The same scheme was revisited by De and Pryor ${ }^{34}$ for the double group IRs with the inclusion of the spin-orbit coupling.

Since WZ has twice more atoms in the primitive unit cell than $\mathrm{ZB}$, the number of energy bands in the $\Gamma$-point is also twice as large. Considering the $s p^{3}$ hybridization, without spin, ZB has 8 energy bands while WZ has 16 . However, in the [111] direction, the $\mathrm{ZB}$ unit cell is two times larger than the usual face-centered cubic (FCC) unit cell. ${ }^{31}$ In the IRs scheme mentioned above, the presence of energy bands with $L$ symmetry takes into account the mismatch in the number of atoms for the usual unit cells. The reason for the appearance of the $L$ symmetry is the fact that ZB [111] growth direction is directed towards the $L$-point, as displayed in Fig. 2(a), hence this point is mapped out in the $\Gamma$-point. Figure 2(b) displays the first Brillouin zone (FBZ) for the WZ structure.

Among all the IRs presented in Refs. 31 and 34, we considered only a small subset. Displayed in Fig. 3, this subset comprises the lower conduction band and the top three valence bands, whose minimum and maxima are assigned to the $\Gamma$-point in both structures. This particular choice of energy bands subset stands because we want to study the mixing of states and the nonparabolicity of the valence band, important effects in the interband transitions of semiconductor systems. The price that is paid in considering only a small subset is the accuracy of the Hamiltonian for a fraction (10\%-20\%) of the FBZ around the $\Gamma$-point. But since the major effects in the electronic and optical properties of the semiconductor are ruled by the carriers in the neighborhood of the band minima, this is a reasonable choice.

The basis states for the IRs of the considered bands are presented in Eqs. (1) and (2) for ZB and WZ, respectively,

$$
\begin{aligned}
& \Gamma_{1 c}^{Z B} \sim x^{2}+y^{2}+z^{2}, \\
& \Gamma_{15 v}^{Z B} \sim(x, y, z),
\end{aligned}
$$
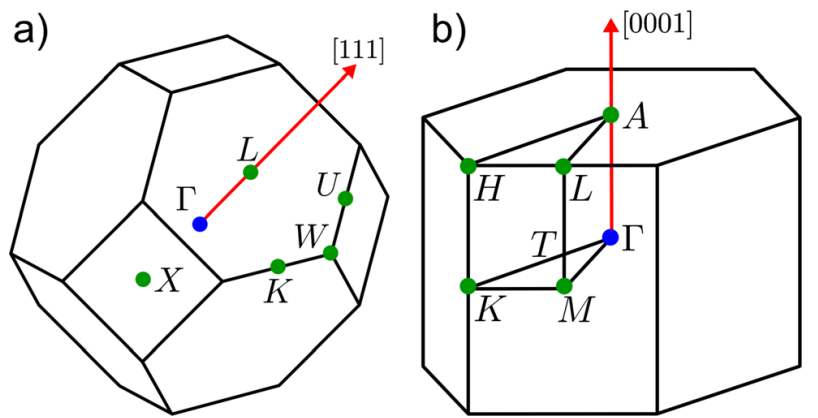

FIG. 2. Usual (a) ZB and (b) WZ first Brillouin zones and their respective high symmetry points. The arrows (red lines) represent the growth directions, the same in Fig. (1). For ZB, the [111] direction is directed towards the L-point. 


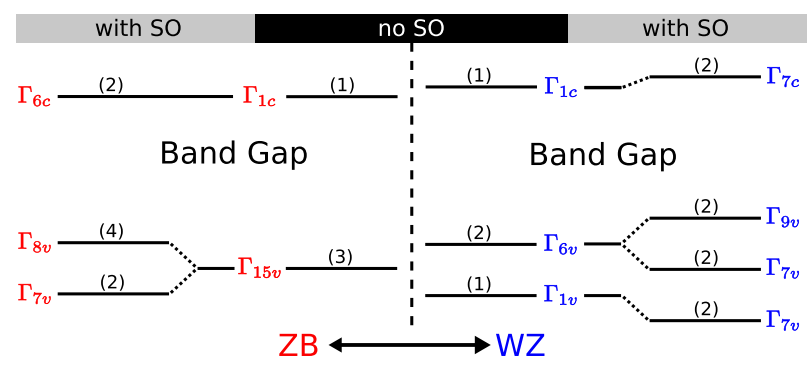

FIG. 3. The subset of IRs considered in this formulation with and without the spin-orbit (SO) coupling. The numbers in parentheses are the degeneracy of the IRs. The notation for the IRs follow Refs. 31 and 34.

$$
\begin{aligned}
& \Gamma_{1 c}^{W Z} \sim x^{2}+y^{2}+z^{2}, \\
& \Gamma_{6 v}^{W Z} \sim(x, y), \\
& \Gamma_{1 v}^{W Z} \sim z .
\end{aligned}
$$

Although the IRs belong to different symmetry groups $\left(T_{d}\right.$ and $C_{6 v}$ ), the basis states transform as the usual $x, y$, and $z$ cartesian coordinates for the valence bands and the scalar $x^{2}+y^{2}+z^{2}$ for the conduction band in both crystal structures. This information is crucial to represent $\mathrm{WZ}$ and $\mathrm{ZB}$ with the same Hamiltonian and is the essential insight of our formulation.

\section{THEORETICAL MODEL}

\section{A. $\mathbf{k} \cdot \mathbf{p}$ and strain Hamiltonian}

In order to develop our Hamiltonian, it is convenient to describe the ZB structure in a coordinate system that has the $z$ axis parallel to the growth direction. In other terms, both phases will be described in the basis set that is normally used to describe wurtzite systems. This coordinate system is the primed one presented in Fig. 4. Even though the choice of the coordinate system is arbitrary, it alters the elements in the Hamiltonian. For example, in the unprimed coordinate system, the $k_{z}$ direction is directed towards the $X$-point but in the primed coordinate system, it is directed towards the $L$-point. Thus, an anisotropy between the $k_{x}$ and $k_{z}$ directions must
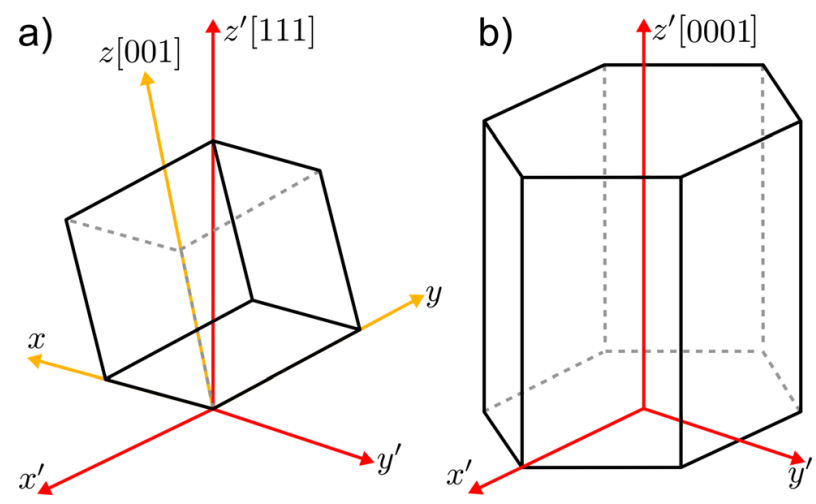

FIG. 4. (a) ZB conventional unit cell with two different coordinate systems. (b) WZ conventional unit cell with its common coordinate system. The [111] growth direction for ZB structure passes along the main diagonal of the cube and is represented in the primed coordinate system. appear in the ZB Hamiltonian since these directions are not reaching equivalent points in the reciprocal space anymore.

Considering the single group formulation, the choice of the coordinate system defines the symmetry operation matrices used to derive the momentum matrix elements. It would be necessary to recalculate the Hamiltonian terms for the ZB structure. However, the energy bands we consider here are exactly the ones customary used in the ZB [001] Hamiltonian. Instead of recalculating the terms for $Z B$ [111] $k \cdot p$ Hamiltonian, it is possible, and also useful, to apply a basis rotation to the $\mathrm{ZB}$ [001] matrix. This rotation procedure is well described in the paper of Park and Chuang. ${ }^{35}$

Since the lattice parameters for $\mathrm{WZ}$ and $\mathrm{ZB}$ structures are not necessarily matched, the strain Hamiltonian is also considered. Due to its familiar form with the k.p terms, it is described in this section. The basis rotation is also applied to obtain the ZB strain matrix in the [111] direction.

The basis set for both crystal structures in the primed coordinate system (the prime will not appear in the notation for simplicity) is given by

$$
\begin{aligned}
& \left|c_{1}\right\rangle=-\frac{1}{\sqrt{2}}|(X+i Y) \uparrow\rangle, \\
& \left|c_{2}\right\rangle=\frac{1}{\sqrt{2}}|(X-i Y) \uparrow\rangle, \\
& \left|c_{3}\right\rangle=|Z \uparrow\rangle, \\
& \left|c_{4}\right\rangle=\frac{1}{\sqrt{2}}|(X-i Y) \downarrow\rangle, \\
& \left|c_{5}\right\rangle=-\frac{1}{\sqrt{2}}|(X+i Y) \downarrow\rangle, \\
& \left|c_{6}\right\rangle=|Z \downarrow\rangle, \\
& \left|c_{7}\right\rangle=i|S \uparrow\rangle, \\
& \left|c_{8}\right\rangle=i|S \downarrow\rangle .
\end{aligned}
$$

In a first approximation, the interband interaction is not taken into account explicitly here, thus the conduction band is a single band model for spin-up and spin-down reading as

$$
\begin{aligned}
E_{C}(\vec{k})= & E_{g}+E_{0}+\frac{\hbar^{2}}{2 m_{e}^{\|}} k_{z}^{2}+\frac{\hbar^{2}}{2 m_{e}^{\perp}}\left(k_{x}^{2}+k_{y}^{2}\right) \\
& +a_{c \|} \varepsilon_{z z}+a_{c \perp}\left(\varepsilon_{x x}+\varepsilon_{y y}\right),
\end{aligned}
$$

where $E_{g}$ is the band gap, $E_{0}$ is the energy reference at $\vec{k}=$ $0, m_{e}^{\|, \perp}$ are the electron effective masses, and $a_{c \|, \perp}$ are the conduction band deformation potentials. The symbols $\|$ and $\perp$ stands for the direction parallel and perpendicular do the $z$ axis, respectively. In the $\mathrm{ZB}$ structure, however, $a_{c \|, \perp}$ and $m_{e}^{\|, \perp}$ have the same value.

The Hamiltonian for $\mathrm{WZ}$ and $\mathrm{ZB}$ valence band is given by

$$
H_{V}(\vec{k})=\left[\begin{array}{cccccc}
F & -K^{*} & -H^{*} & 0 & 0 & 0 \\
-K & G & H & 0 & 0 & \Delta \\
-H & H^{*} & \lambda & 0 & \Delta & 0 \\
0 & 0 & 0 & F & -K & H \\
0 & 0 & \Delta & -K^{*} & G & -H^{*} \\
0 & \Delta & 0 & H^{*} & -H & \lambda
\end{array}\right]
$$


and the matrix terms are defined as

$$
\begin{aligned}
F= & \Delta_{1}+\Delta_{2}+\lambda+\theta \\
G= & \Delta_{1}-\Delta_{2}+\lambda+\theta \\
\lambda= & A_{1} k_{z}^{2}+A_{2}\left(k_{x}^{2}+k_{y}^{2}\right) \\
& +D_{1} \varepsilon_{z z}+D_{2}\left(\varepsilon_{x x}+\varepsilon_{y y}\right), \\
\theta= & A_{3} k_{z}^{2}+A_{4}\left(k_{x}^{2}+k_{y}^{2}\right) \\
& +D_{3} \varepsilon_{z z}+D_{4}\left(\varepsilon_{x x}+\varepsilon_{y y}\right), \\
K= & A_{5} k_{+}^{2}+2 \sqrt{2} A_{z} k_{-} k_{z} \\
& +D_{5}^{(1)}\left(\varepsilon_{x x}-\varepsilon_{y y}\right)+D_{5}^{(2)} 2 i \varepsilon_{x y}, \\
H= & A_{6} k_{+} k_{z}+A_{z} k_{-}^{2} \\
& +D_{6}\left(\varepsilon_{x z}+i \varepsilon_{y z}\right)+D_{z}\left(\varepsilon_{x x}-\varepsilon_{y y}\right), \\
\Delta= & \sqrt{2} \Delta_{3},
\end{aligned}
$$

where $k_{\alpha}(\alpha=x, y, z)$ are the wave vectors in the primed coordinate system, $A_{i}(i=1, \ldots, 6, z)$ are the holes effective mass parameters, $\Delta_{1}$ is the crystal field splitting energy in WZ, $\Delta_{2,3}$ are the spin-orbit coupling splitting energies, $k_{ \pm}=k_{x} \pm i k_{y}, D_{i}$ 's are the valence band deformation potentials, and $\varepsilon_{i j}(i, j=x, y, z)$ are the components of the strain tensor.

It is important to notice that the parameter $A_{z}$ appears in the matrix elements to regain the original isotropic symmetry of the $\mathrm{ZB}$ band structure in the new coordinate system. In the regions of WZ crystal structure, this parameter is zero and the matrix is exactly the canonical in use for WZ crystals.

Although this is not the usual way to describe ZB crystals, all the parameters in the matrix can be related to the familiar $\gamma_{i}(i=1,2,3)$ and $\Delta_{S O}$, as shown above

$$
\begin{aligned}
& \Delta_{1}=0, \\
& \Delta_{2}=\Delta_{3}=\frac{\Delta_{S O}}{3}, \\
& A_{1}=-\gamma_{1}-4 \gamma_{3}, \\
& A_{2}=-\gamma_{1}+2 \gamma_{3}, \\
& A_{3}=6 \gamma_{3}, \\
& A_{4}=-3 \gamma_{3}, \\
& A_{5}=-\gamma_{2}-2 \gamma_{3}, \\
& A_{6}=-\sqrt{2}\left(2 \gamma_{2}+\gamma_{3}\right), \\
& A_{z}=\gamma_{2}-\gamma_{3} .
\end{aligned}
$$

In the same way as the $A_{z}$ parameter appears in $\mathrm{k} \cdot \mathrm{p}$ matrix, some extra deformation potential terms were appears to allow the use of the same strain Hamiltonian for both crystal structures. The deformation potential $D_{5}$ was split in two parts because the strain tensor $\varepsilon_{x y}$ is not present in the ZB structure. For WZ, $D_{5}^{(1)}=D_{5}^{(2)}$. Also, the $D_{z}$ deformation potential takes into account the non existing term $\varepsilon_{x x}-\varepsilon_{y y}$ in the WZ structure.

The deformation potentials $D_{i}$ 's are related to the ZB ones

$$
\begin{aligned}
D_{1} & =a_{v}+\frac{2 d}{\sqrt{3}}, \\
D_{2} & =a_{v}-\frac{d}{\sqrt{3}}, \\
D_{3} & =-\sqrt{3} d, \\
D_{4} & =\frac{3 d}{2 \sqrt{3}}, \\
D_{5}^{(1)} & =-\frac{b}{2}-\frac{d}{\sqrt{3}}, \\
D_{5}^{(2)} & =0, \\
D_{6} & =0, \\
D_{z} & =-\frac{b}{2}+\frac{d}{2 \sqrt{3}}, \\
a_{c \|} & =a_{c \perp}=a_{c} .
\end{aligned}
$$

One may also argue that this formulation is very similar to the $\mathrm{WZ}$ phase. The insight here is to consider $\mathrm{ZB}$ as a $\mathrm{WZ}$ structure without the crystal field splitting energy. Since the WZ structure is less symmetric than $\mathrm{ZB}$, as mentioned in Sec. II A, it is possible to represent the $\mathrm{ZB}$ parameters with the WZ ones.

The coordinate system rotation of the Hamiltonian also applies to the strain tensor components. Considering biaxial strain, it is possible to obtain effective values for $C_{13}^{(z)}$ and $C_{33}^{(z)}$ for $\mathrm{ZB}$ in the primed coordinate system. The upper script $(z)$ is to differ from the WZ structure. These effective values are

$$
\begin{aligned}
& C_{13}^{(z)}=C_{11}^{(z)}+2 C_{12}^{(z)}-2 C_{44}^{(z)}, \\
& C_{33}^{(z)}=C_{11}^{(z)}+2 C_{12}^{(z)}+4 C_{44}^{(z)} .
\end{aligned}
$$

Thus, we have a single set of expressions to describe biaxial strain in the primed coordinate system for both $\mathrm{ZB}$ and WZ crystal structures

$$
\begin{gathered}
\varepsilon_{x x}=\varepsilon_{y y}=\frac{a_{0}-a}{a}, \\
\varepsilon_{z z}=-\frac{2 C_{13}^{(z, w)}}{C_{33}^{(z, w)}} \varepsilon_{x x}, \\
\varepsilon_{y z}=\varepsilon_{z x}=\varepsilon_{x y}=0 .
\end{gathered}
$$

Since deformation potentials and elastic stiffness constants for WZ InP are not yet available in the literature, we will consider here that the strain effect appears only in the ZB structure. This assumption is not totally unrealistic because $\mathrm{WZ}$ is the dominant phase in the NW.

\section{B. Spontaneous and piezoelectric polarization}

Piezoelectric polarization appears when a crystal is subjected to strain. In ZB semiconductors grown along the [111] direction, the magnitude of the piezoelectric polarization, in the unprimed coordinate system of Fig. 4, is given by ${ }^{36}$

$$
P_{i}=2 e_{14} \varepsilon_{j k},
$$


where $e_{14}$ is the piezoelectric constant for ZB materials, $(i, j$, $k)$ are the cartesian coordinates $(x, y, z)$ in a cyclic order, and $\varepsilon_{j k}$ are the strain tensor components.

Applying the coordinate system rotation in the piezoelectric polarization vector components in order to describe them in the primed coordinate system, we obtain the resulting piezoelectric polarization alongside the growth direction

$$
P_{z}=-\frac{2}{\sqrt{3}} e_{14}\left[1+\frac{2 C_{13}^{(z)}}{C_{33}^{(z)}}\right] \varepsilon_{x x},
$$

where $\varepsilon_{x x}$ is given by the expression (11).

The spontaneous polarization effect in WZ structure is due to the relative displacement between the cations and anions when the ratio $c / a$ is different from the ideal value of the hexagonal close-packed structure.

In a heterostructure, the effect of the different polarizations in each region creates an electric field through the whole structure. The net electric field in a determined layer, $i$, due to spontaneous and piezoelectric polarizations in the system is given by ${ }^{37}$

$$
E_{i}=\frac{\sum_{j=1}^{N}\left(P_{j}-P_{i}\right) \frac{l_{j}}{\varepsilon_{j}}}{\varepsilon_{i} \sum_{j=1}^{N} \frac{l_{j}}{\varepsilon_{j}}},
$$

where $j$ sums all over the layers in the heterostructure with polarization $P$, dielectric constant $\varepsilon$, and length $l$.

\section{Effective mass equation in reciprocal space}

In the NW, there are several regions of $\mathrm{WZ}$ and $\mathrm{ZB}$ phases that create confinement profiles for the carriers due to the band offset in the polytypical interface. Each region, or crystalline structure, is comprised by their unique set of parameters. The envelope function approximation ${ }^{26,38}$ is applied to couple the different crystal structures alongside the growth direction $(z)$ in the NW. In each region, the wave function is expanded in terms of the Bloch functions of the corresponding polytype. Thus, the wave function of the whole system is given by

$$
\psi(\vec{r})=\sum_{l} e^{i(\vec{k} \cdot \vec{r})} g_{l}(z) u_{l}^{(W Z, Z B)}(\vec{r})
$$

where $g_{l}(\vec{r})$ are the envelope functions of the $l$-th basis state.

Considering different Bloch functions for each region, since they are not eigenstates of the bulk Hamiltonian, the parameters vary alongside the growth direction, making it possible to use matrix (5), for both crystal structures. Moreover, since each crystal structure dictates its symmetry to their respective Bloch functions, some matrix elements can be forbidden by symmetry in the region of a certain crystalline phase. For example, the $A_{z}$ parameter is zero in WZ regions, whereas the $\Delta_{1}$ parameter is zero in $\mathrm{ZB}$ regions.

To represent the growth dependence of the Hamiltonian parameters and envelope functions, the plane wave expansion is used. This formalism considers the periodicity (periodic boundary conditions) of the whole system allowing the expansion of the growth dependent functions in Fourier coefficients

$$
U(z)=\sum_{K} U_{K} e^{i K z}
$$

where $U_{K}$ are the Fourier coefficients of the function $U(z)$ and $K$ is a reciprocal lattice vector of the whole system in $z$ direction.

Since the Fourier expansion has a $z$ dependent exponential, its combination with the exponential part of Eq. (17) induces the change $\vec{k} \rightarrow \vec{k}+K \hat{z}$ in the expressions (4) and $(5)$, completing the plane wave expansion. ${ }^{27,30}$

\section{RESULTS AND DISCUSSION}

In order to test the model, it was applied to a polytypical $\mathrm{WZ} / \mathrm{ZB} / \mathrm{WZ}$ quantum well of InP. Although a real NW is composed by several regions with different sizes of $\mathrm{WZ}$ and $\mathrm{ZB}$, the physical trends of the polytypical interface can be extracted from a single well structure. We choose the InP compound basically for two reasons: the small spin-orbit energy makes easier to fit the matrix parameters to the effective mass values given in the paper of De and Pryor ${ }^{34}$ for the WZ polytype and also because the InP NWs can be found in a great number of studies in the present literature. ${ }^{13,39-45}$ The effects of lateral confinement are neglected in a first approach, assuming NWs with large lateral dimensions. Strain, piezoelectric, and spontaneous polarization are also included in the single well system.

When both crystal structures are put side by side, a band offset is created at the interface, originating a confinement profile. However, comparing the band edges (at $\vec{k}=0$ ) of different crystal phases is a difficult task. In $\mathrm{ZB}$, the angular momentum basis gives us a natural description of the band edges since the diagonalization of spin also occurs in this basis. For WZ, the basis that diagonalizes the Hamiltonian at $\vec{k}=0$ is dependent on the parameters $\Delta_{1,2,3}$. Therefore, neither of the bases are appropriate to describe both phases together.

The basis set presented in Eq. (3) preserves the spin and also leaves the crystal field split energy, $\Delta_{1}$, diagonal as well as the spin-orbit term $\Delta_{2}$. This is a very convenient basis since $\Delta_{1}$ is responsible for the highest symmetry break in $\mathrm{WZ}$ and this is the dominant structure in NWs. Also, for the case of InP, $\Delta_{1}$ is almost 9 times larger than $\Delta_{2,3}$ and represents the biggest contribution for the valence band profile in WZ phase.

Figure 5 exhibits the unstrained energy profile at $\vec{k}=0$ for WZ/ZB InP in two different schemes: in the left, the energies for the diagonalized Hamiltonian of the system and in the right, the diagonal terms of Eqs. (4) and (5) in the basis set (3), including the band mismatch. Notice that in the right scheme, the $\Delta_{3}$ is not taken into account since it is offdiagonal. The notation in the right side refers to the diagonal matrix elements of the states in the basis set (3), which means

$$
\begin{aligned}
& \left|c_{1,4}\right\rangle \Rightarrow\left\langle c_{1,4}|H| c_{1,4}\right\rangle=\Delta_{1}(z)+\Delta_{2}(z)+\Delta E_{v}(z), \\
& \left|c_{2,5}\right\rangle \Rightarrow\left\langle c_{2,5}|H| c_{2,5}\right\rangle=\Delta_{1}(z)-\Delta_{2}(z)+\Delta E_{v}(z), \\
& \left|c_{3,6}\right\rangle \Rightarrow\left\langle c_{3,6}|H| c_{3,6}\right\rangle=\Delta E_{v}(z), \\
& \left|c_{7,8}\right\rangle \Rightarrow\left\langle c_{7,8}|H| c_{7,8}\right\rangle=E_{g}(z)+E_{0}(z)+\Delta E_{c}(z),
\end{aligned}
$$



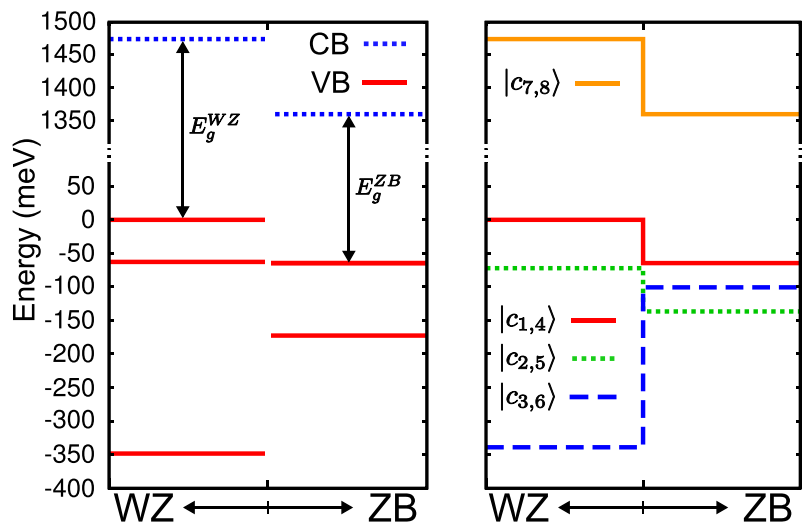

FIG. 5. Unstrained energy profile at the polytypical interface at $\vec{k}=0$. Left side: Band edge energies of the system. Right side: Diagonal terms of the Hamiltonian Eqs. (4) and (5). The $\left|c_{i}\right\rangle, i=1, \ldots, 8$ represents the elements of the basis set (3). The band mismatch between $\mathrm{WZ}$ and $\mathrm{ZB}$ phases is taken from Ref. 34.

where $\Delta E_{c}(z)$ and $\Delta E_{v}(z)$ are the band offsets for conduction and valence band, respectively.

\section{A. Strain effects}

In all performed calculations, the entire length of the system is set to $500 \AA$, and the width of the $\mathrm{ZB}$ region, $l$, is variable. The $\mathrm{k} \cdot \mathrm{p}$ and strain parameters for $\mathrm{ZB}$ and $\mathrm{WZ} \mathrm{InP}$ can be found in Tables I and II, respectively.

Figure 6 shows the single well potentials with and without the effect of strain for the calculations. Since the potential profiles exhibits a type-II behavior, we expect a spatial separation of the carriers: electrons are more likely to be in the $\mathrm{ZB}$ region and the holes in the $\mathrm{WZ}$ region.

TABLE I. $\mathrm{k} \cdot \mathrm{p}$ parameters used for InP in the calculations.

\begin{tabular}{lcc}
\hline \hline Parameter & ZB InP (Ref. 46) & WZ InP (Ref. 34) \\
\hline Lattice constant (A) & 5.8697 & 4.1505 \\
$a$ & - & 6.7777 \\
$c$ & & \\
Energy parameters (eV) & 1.4236 & 1.474 \\
$E_{g}$ & 0 & 0.303 \\
$\Delta_{1}$ & 0.036 & 0.036 \\
$\Delta_{2}=\Delta_{3}$ & & \\
Conduction band effective masses & 0.0795 & 0.105 \\
$m_{e}^{\|} / m_{0}$ & 0.0795 & 0.088 \\
$m_{e}^{\perp} / m_{0}$ & & \\
Valence band effective & & \\
mass parameters (units of $\left.\frac{\hbar^{2}}{2 m_{0}}\right)$ & -13.4800 & -10.7156 \\
$A_{1}$ & -0.8800 & -0.8299 \\
$A_{2}$ & 12.6000 & 9.9301 \\
$A_{3}$ & -6.3000 & -5.2933 \\
$A_{4}$ & -5.8000 & 5.0000 \\
$A_{5}$ & -7.4953 & 1.5000 \\
$A_{6}$ & -0.5000 & 0 \\
$A_{z}$ & & \\
\hline \hline
\end{tabular}

TABLE II. Strain parameters for ZB InP used in the calculations.

\begin{tabular}{lc}
\hline \hline Parameter & ZB InP (Ref. 46) \\
\hline Deformation potentials (eV) & -6.3735 \\
$D_{1}$ & 2.2868 \\
$D_{2}$ & 8.6603 \\
$D_{3}$ & -4.3301 \\
$D_{4}$ & 3.8868 \\
$D_{5}^{(1)}$ & 0 \\
$D_{5}^{(2)}$ & 0 \\
$D_{6}$ & -0.4434 \\
$D_{z}$ & -6.0 \\
$a_{c}$ & -6.0 \\
$a_{c \perp}$ & \\
Elastic stiffness constant (GPa) & 101.1 \\
$C_{11}$ & 56.1 \\
$C_{12}$ & 45.6 \\
$C_{44}$ & \\
\hline \hline
\end{tabular}

The strain considered here, $-0.8 \%$, is a intermediate value between two data available in the literature from $a b$ initio calculations. Reference 44 shows that the deviation between the lattice constant of ZB[111] and WZ[0001] is $-1.3 \%$ and Ref. 43 shows $-0.3 \%$. Also, Ref. 42 suggests a difference slighter than $0.5 \%$ between the lattice constants of the two polytypes. The effect of strain shallows the potential wells in the conduction and valence bands, reducing the confinement of the carriers. We expect to have less confined states for the strained potential compared to the unstrained one.

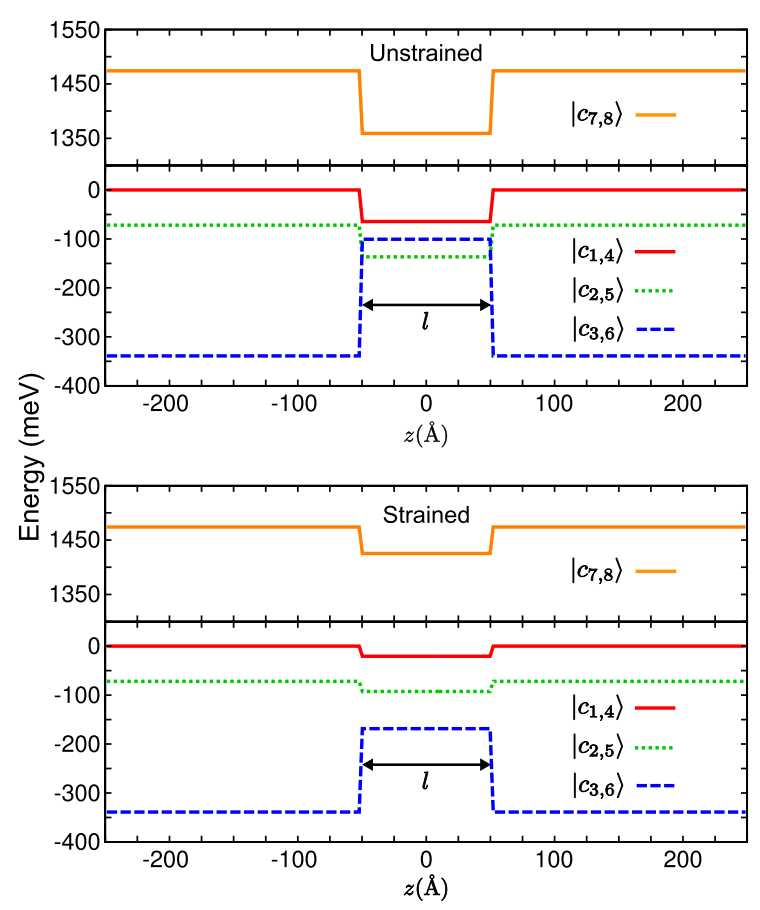

FIG. 6. Diagonal potential profile of the Hamiltonian for the polytypical InP system with and without strain. The width of the $\mathrm{ZB}$ region, $l$, can change but the whole system's dimension remains constant with $500 \AA$. 
For both unstrained and strained cases, the valence band states in $\vec{k}=0$ are either heavy holes $(\mathrm{HH})$ or light holes $(\mathrm{LH})$. There is no crystal-field split-off hole $(\mathrm{CH})$ states in the energy range considered here. This characterization of the energy bands was made following Chuang and Chang notation, ${ }^{47}$ the usual one for $\mathrm{WZ}$ compounds. The resulting band structures for some values of $l$ can be found in the supplemental material. ${ }^{52}$

The information of the energy states' composition can reveal important trends in the luminescence spectra for this kind of system. For example, at $\vec{k}=0$, the dominant symmetry of the energy states belongs to $(x, y)$, which means that the luminescence spectra are more intense perpendicular to the growth direction. However, experimental measurements ${ }^{41}$ indicate that the intensities perpendicular and parallel to the growth direction are almost similar. Therefore, we expect that the contribution for the parallel luminescence comes from the states at $\vec{k} \neq 0$. For $\vec{k}$ points away from the $\Gamma$-point, there is a stronger mixing between all the basis states, allowing more considerable contribution from $z$ symmetry.

The effect of the $\mathrm{ZB}$ region width for both strained and unstrained cases is summarized in Fig. 7 for the conduction and valence band states at $\vec{k}=0$. Increasing the value of $l$, we find that the number of confined states in the conduction band increases. On the other hand, for the valence band, the number of confined states decreases because the WZ region's width also decreases. Nevertheless, the effect of the variation of $l$ is more significant for the conduction band since the electron effective mass in $\mathrm{ZB}\left(m_{e}^{*} / m_{0}=0.0795\right)^{46}$ is smaller than the heavy hole mass of $\mathrm{WZ}\left(m_{H H}^{\|} / m_{0}=1.273\right.$ and $\left.m_{H H}^{\perp} / m_{0}=0.158\right) .^{34}$

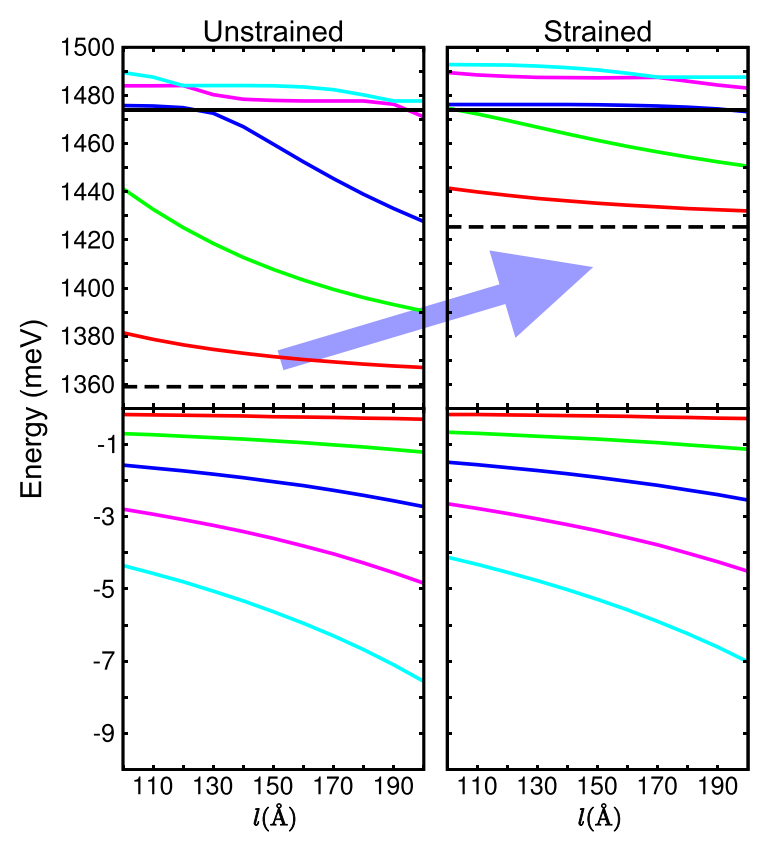

FIG. 7. The first 5 states of the conduction and valence bands at $\vec{k}=0$ as a function of the $\mathrm{ZB}$ region width $l$. The solid (black) lines indicate the top of the conduction band well and the dashed (black) lines indicate the bottom. The arrow (blue) indicates the blueshift induced in interband energy transition due to strain effects.
It is possible to observe the same confinement trends in the strained case. However, the number of confined states for the strained profile compared to the unstrained one is smaller, since the bottom of the well in the conduction band has a higher value. This energy displacement in the conduction band induces a blueshift in the interband transition energy peak of, approximately, $60 \mathrm{meV}$.

\section{B. Polarization effects}

Strain also gives rise to the piezoelectric polarization due to the relative displacements between cations and anions in the crystal structure. For the InP ZB, the value for the piezoelectric constant used was $e_{14}=0.035 \mathrm{C} / \mathrm{m}^{2}{ }^{48}$ Also, for WZ compounds, the spontaneous polarization is present. This parameter is unknown for WZ InP, however, Ref. 42 suggests that this value is smaller than that of $\mathrm{InN}$ $\left(-0.03 \mathrm{C} / \mathrm{m}^{2}\right)$. In an attempt to estimate this value for $\mathrm{InP}$, we performed the band structure calculations considering a range of values for $P_{s p}$. For both crystal structures, the value used for static dielectric constant was 12.5, taken from the Ioffe Institute's New Semiconductor Materials electronic archive website. ${ }^{49}$

The energy of the first 5 conduction and valence band states at $\vec{k}=0$ as a function of spontaneous polarization in $\mathrm{WZ} \mathrm{InP}$ for three different $\mathrm{ZB}$ region widths is presented in Fig. 8. For $l=160 \AA$ and $l=200 \AA$, there

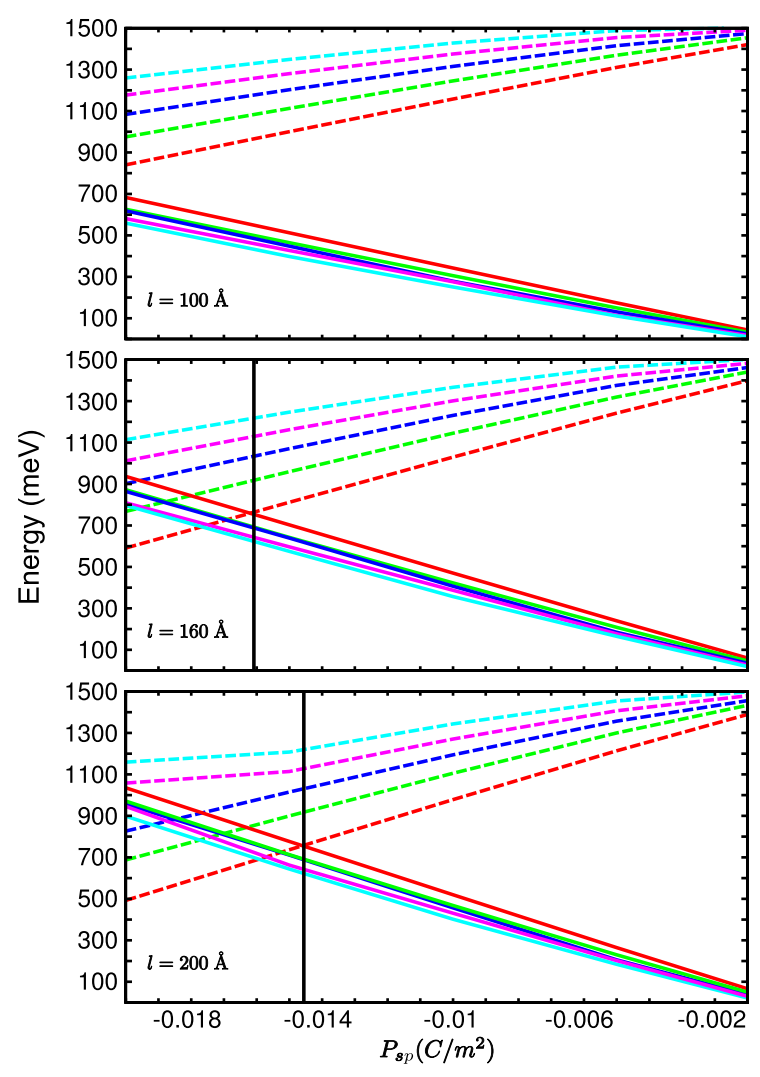

FIG. 8. The first 5 states of the conduction (dashed line) and valence (solid line) bands at $\vec{k}=0$ as a function of WZ spontaneous polarization $P_{s p}$ (varying from $-0.02 \mathrm{C} / \mathrm{m}^{2}$ to $-0.001 \mathrm{C} / \mathrm{m}^{2}$ ). Notice the crossing of valence and conduction bands indicating a negative gap in the system. The vertical lines indicate the point where the energy gap of the system changes sign. 

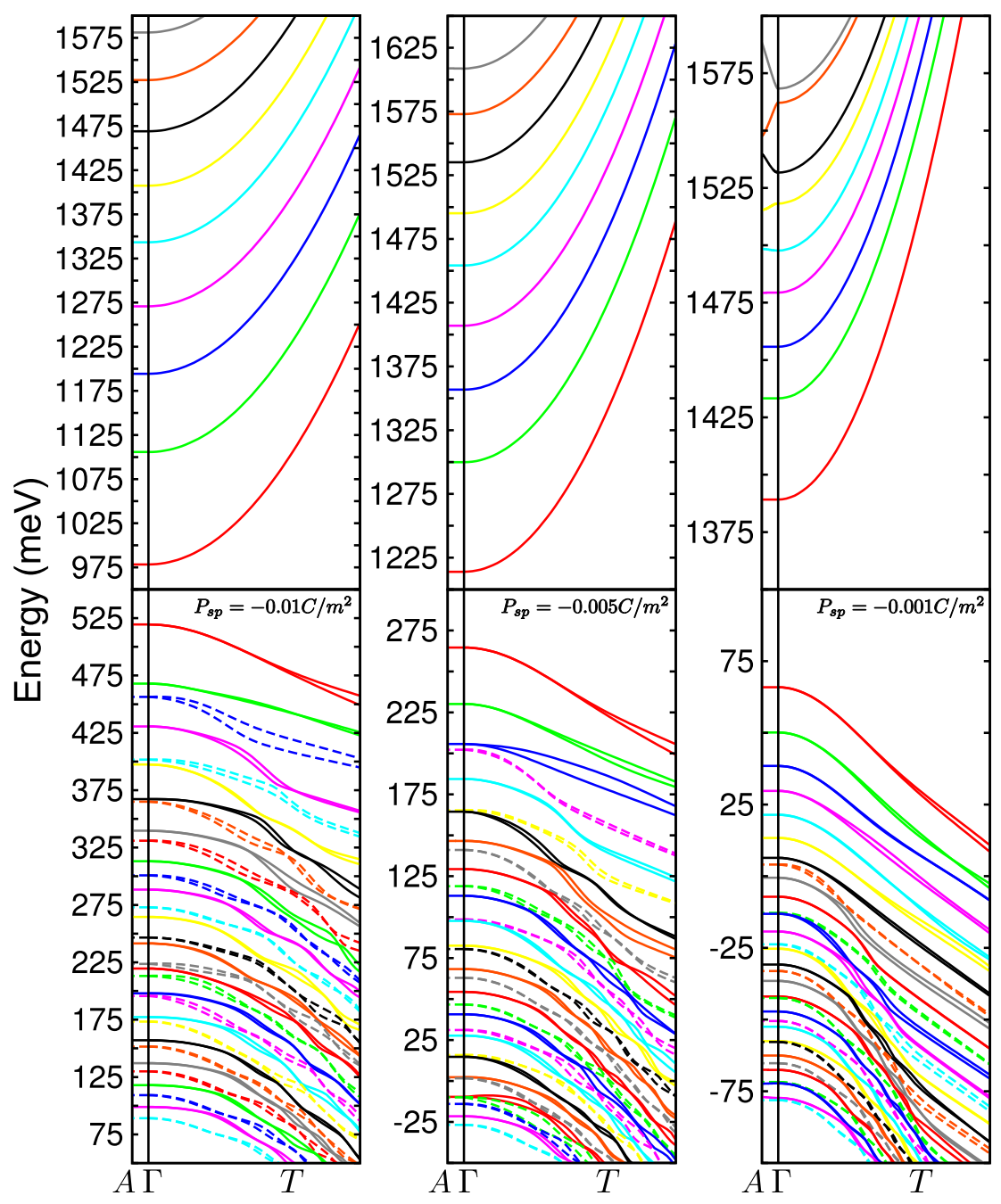

FIG. 9. Band structure calculations for three different values of $P_{s p}$. In the valence band, the solid lines represent $\mathrm{HH}$ states and the dashed lines represent LH states. The spin-splitting of the valence energy bands is due to the field induced asymmetry.

is a crossing between the conduction and valence band states, denoted by the vertical line. This is not observed experimentally, therefore, we consider this region forbidden. Then, from the allowed range of spontaneous polarization (right side of the vertical line in Fig. 8), we considered the values $-0.01 \mathrm{C} / \mathrm{m}^{2},-0.005 \mathrm{C} / \mathrm{m}^{2}$, and $-0.001 \mathrm{C} / \mathrm{m}^{2}$ to analyze the polarization effects in the band structure.

Fixing the $\mathrm{ZB}$ region at $200 \AA$, we calculated the band structures for the three values of $P_{s p}$ discussed above. These results are shown in Fig. 9. From bottom to top, the conduction energy bands are nominated as EL1-EL9, composed of $\left|c_{7,8}\right\rangle$ states. In the valence band, the solid lines are HH states and the dashed lines are LH states. Since the net polarization potential increases with the value of $P_{s p}$, the energy range is different for the three cases analyzed above. Also, the number of confined states decreases as the spontaneous polarization decreases. On the other hand, the energy difference between the conduction and valence band ground state, the energy gap of the system, increases as the spontaneous polarization decreases, blueshifting the interband energy transitions.
Higher values of $P_{s p}$ induces stronger mixing of states. The anticrossings and also the spin splitting in the valence sub bands can be observed. The strength of the resulting electric field not only increases the mixing of $\mathrm{HH}$ and $\mathrm{LH}$ states but also increases the value of the spin splitting in each sub band. This spin splitting is the well known Rashba effect ${ }^{50}$ due to the potential inversion asymmetry, even though the term $\alpha(\vec{\sigma} \times \vec{k}) \cdot \vec{E}$ does not appear explicitly in the Hamiltonian. ${ }^{51}$

\section{Spatial carriers separation}

The effect of piezoelectric and spontaneous polarization also induce carriers' spatial separation. This effect, in the probability densities in $\vec{k}=0$, can be seen in Fig. 10 . The lowest four states of the conduction band and the highest four states of the valence band are presented. At $\vec{k}=0$, the wave functions of spin-up and spin-down are degenerated. We can see that the overlap increases for more excited states, also blueshifting the energy peak in the interband transitions from the fundamental gap of the system. Since the potential profile is not completely even or odd, the envelope functions no longer have well defined parities. 

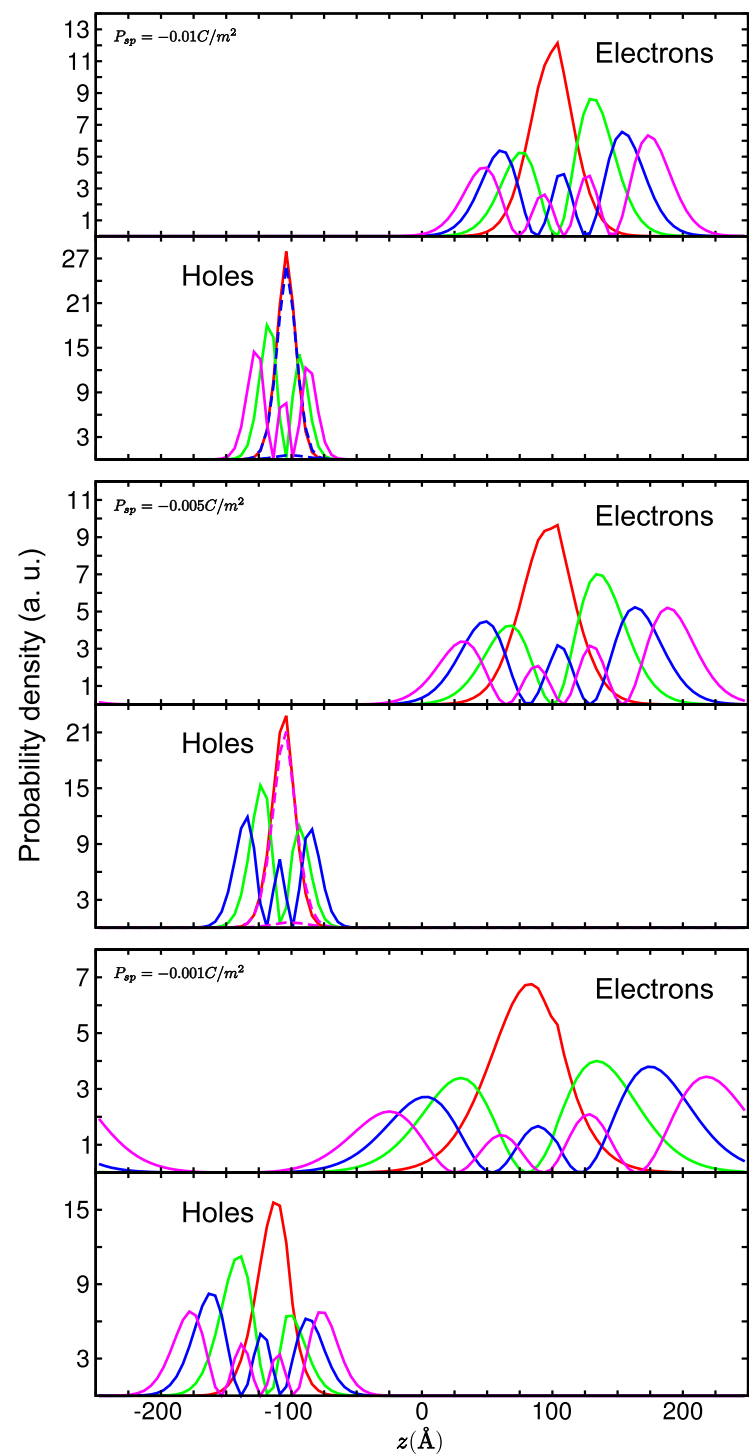

FIG. 10. Probability densities at $\vec{k}=0$ for the lowest four states of the conduction band and the highest four states of the valence band of Fig. 9. The solid lines are the HH states and dashed lines represent the LH states.

\section{CONCLUSIONS}

The basic result of this study is the theoretical model based on the $\mathrm{k} \cdot \mathrm{p}$ method and group theory concepts to calculate band structures of WZ/ZB polytypical systems in the vicinity of the band edge. The method allows us to describe in the same matrix Hamiltonian the $\mathrm{ZB}$ and WZ structures, with $k_{z}$ along the [111] and [0001] directions, respectively. Since the WZ structure is less symmetric, the ZB parameters are assigned to the $\mathrm{WZ}$ ones. Our method not only is able to describe the $\mathrm{k} \cdot \mathrm{p}$ terms of the Hamiltonian but also includes the strain and polarization (spontaneous and piezoelectric) effects.

Extracting the parameters of WZ InP from Ref. 34, we applied our model to a WZ/ZB/WZ single well in order to understand the physics of the polytypical interface. The potential profile at the interface WZ/ZB is type-II, whose feature is the spatial separation of carriers. The performed calculations in this study hold this characteristic.
Due to the lack of parameters in the literature for $\mathrm{WZ}$ $\mathrm{InP}$, only the strain effect in the $\mathrm{ZB}$ region was considered here. This seems to be a reasonable consideration since the WZ structure is the dominant phase in NWs structures. However, such strain parameters would be fundamental in a system that the stable lattice constant is an intermediate value between $\mathrm{WZ}$ and ZB InP lattice parameters.

Within the limitation of strain, the piezoelectric polarization was also considered in the $\mathrm{ZB}$ region. For the $\mathrm{WZ}$ region, only the spontaneous polarization appears. Since there is no value in the literature for the spontaneous polarization of WZ InP, a range of values were considered in the simulations. Some of these values, however, induces a negative gap in the system. There is no data in the literature that corroborates this effect.

The proposed model, jointly with the obtained results, proved to be useful in the study of electronic band structures of WZ/ZB polytypical systems, such as NWs. Exploring the opportunities of band gap engineering, considering not only different compounds but also different crystal structures, could lead to the development of novel nanodevices.

\section{ACKNOWLEDGMENTS}

The authors acknowledge financial support from the Brazilian funding agencies CAPES, CNPq, and FAPESP.

${ }^{1}$ Y. Cui, Q. Wei, H. Park, and C. M. Lieber, Science 293, 1289 (2001).

${ }^{2}$ F. Patolsky, G. Zheng, and C. M. Lieber, Nanomedicine 1, 51 (2006).

${ }^{3}$ G. Zheng, F. Patolsky, Y. Cui, W. U. Wang, and C. M. Lieber, Nat. Biotechnol. 23, 1294 (2005).

${ }^{4}$ M. T. Björk, B. J. Ohlsson, T. Sass, A. I. Persson, C. Thelander, M. H. Magnusson, K. Deppert, L. R. Wallenberg, and L. Samuelson, Appl. Phys. Lett. 80, 1058 (2002).

${ }^{5}$ X. Duan, Y. Huang, Y. Cui, J. Wang, and C. M. Lieber, Nature 409, 66 (2001).

${ }^{6}$ P. J. Pauzauskie, D. J. Sirbuly, and P. Yang, Phys. Rev. Lett. 96, 143903 (2006).

${ }^{7}$ A. B. Greytak, L. J. Lauhon, M. S. Gudiksen, and C. M. Lieber, Appl. Phys. Lett. 84, 4176 (2004).

${ }^{8}$ H. Ng, J. Han, T. Yamada, and P. Nguyen, Nano Lett. 4, 1247 (2004).

${ }^{9}$ J. Goldberger, A. I. Hochbaum, R. Fan, and P. Yang, Nano Lett. 6, 973 (2006).

${ }^{10}$ G. C. Liang, J. Xiang, N. Kharche, G. Klimeck, C. M. Lieber, and M. Lundstrom, Nano Lett. 7, 642 (2007).

${ }^{11}$ R. S. Wagner and W. C. Ellis, Appl. Phys. Lett. 4, 89 (1964).

${ }^{12}$ V. Dubrovskii, G. Cirlin, and V. Ustinov, Semiconductors 43, 1539 (2009).

${ }^{13}$ Y. Kitauchi, Y. Kobayashi, K. Tomioka, S. Hara, K. Hiruma, T. Fukui, and J. Motohisa, Nano Lett. 10, 1699 (2010).

${ }^{14}$ J. Johansson, L. S. Karlsson, C. P. T. Svensson, T. Mårtensson, B. A Wacaser, K. Deppert, L. Samuelson, and W. Seifert, Nature Mater. 5, 574 (2006).

${ }^{15}$ P. Caroff, K. A. Dick, J. Johansson, M. E. Messing, K. Deppert, and L. Samuelson, Nat. Nanotechnol. 4, 50 (2009).

${ }^{16}$ S.-G. Ihn, J.-I. Song, Y.-H. Kim, J. Y. Lee, and I.-H. Ahn, IEEE Trans. Nanotechnol. 6, 384 (2007).

${ }^{17}$ J. Bao, D. C. Bell, F. Capasso, N. Erdman, D. Wei, L. Fröberg, T. Mårtensson, and L. Samuelson, Adv. Mater. 21, 3654 (2009).

${ }^{18}$ J. Bolinsson, P. Caroff, B. Mandl, and K. A. Dick, Nanotechnology 22, 265606 (2011).

${ }^{19}$ C. Thelander, P. Caroff, S. Plissard, A. W. Dey, and K. A. Dick, Nano Lett. 11, 2424 (2011).

${ }^{20}$ K. A. Dick, P. Caroff, J. Bolinsson, M. E. Messing, J. Johansson, K. Deppert, L. R. Wallenberg, and L. Samuelson, Semicond. Sci. Technol. 25, 024009 (2010)

${ }^{21}$ G. Dresselhaus, Phys. Rev. 100, 580 (1955). 
${ }^{22}$ E. O. Kane, Physics of III-V compounds, Semiconductors and Semimetals (Academic, New York, 1966), Vol. 1.

${ }^{23}$ G. E. Pikus, Sov. Phys. JETP 14, 898 (1962).

${ }^{24}$ G. Bir and G. Pikus, Symmetry and Strain-Induced Effects in Semiconductors (Wiley, New York, 1974).

${ }^{25}$ S. L. Chuang and C. S. Chang, Phys. Rev. B 54, 2491 (1996).

${ }^{26} \mathrm{G}$. Bastard and J. A. Brum, IEEE J. Quantum Electron. 22, 1625 (1986).

${ }^{27}$ G. M. Sipahi, R. Enderlein, L. M. R. Scolfaro, and J. R. Leite, Phys. Rev. B 53, 9930 (1996).

${ }^{28}$ S. C. P. Rodrigues, G. M. Sipahi, L. M. R. Scolfaro, and J. R. Leite, Appl. Phys. Lett. 76, 1015 (2000).

${ }^{29}$ L. C. Chuang and C. S. Chang, Semicond. Sci. Technol. 12, 252 (1997).

${ }^{30}$ S. C. P. Rodrigues and G. M. Sipahi, J. Cryst. Growth 246, 347 (2002).

${ }^{31}$ M. Murayama and T. Nakayama, Phys. Rev. B 49, 4710 (1994).

${ }^{32}$ P. E. Faria Junior and G. M. Sipahi, http://arxiv.org/abs/1012.0227 (2010).

${ }^{33}$ M. S. Dresselhaus, G. Dresselhaus, and A. Jorio, Group Theory: Application to the Physics of Condensed Matter (Springer-Verlag, 2008).

${ }^{34}$ A. De and C. E. Pryor, Phys. Rev. B 81, 155210 (2010).

${ }^{35}$ S. H. Park and S. L. Chuang, J. Appl. Phys. 87, 353 (2000).

${ }^{36}$ C. Mailhiot and D. L. Smith, Phys. Rev. B 35, 1242 (1987).

${ }^{37} \mathrm{P}$. Harrison, Quantum Wells, Wires and Dots: Theoretical and Computational Physics of Semiconductor Nanostructures (Wiley, 2006).

${ }^{38}$ G. Bastard, Wave Mechanics Applied to Semiconductor Heterostructures (Halsted, Les Ulis Cedex, France, 1988).

${ }^{39}$ K. Pemasiri, M. Montazeri, R. Gass, L. M. Smith, H. E. Jackson, J. Yarrison-Rice, S. Paiman, Q. Gao, H. H. Tan, C. Jagadish, X. Zhang, and J. Zou, Nano Lett. 9, 648 (2009).
${ }^{40}$ S. Paiman, Q. Gao, H. H. Tan, C. Jagadish, K. Pemasiri, M. Montazeri, H. E. Jackson, L. M. Smith, J. M. Yarrison-Rice, X. Zhang, and J. Zou, Nanotechnology 20, 225606 (2009).

${ }^{41}$ E. G. Gadret, G. O. Dias, L. C. O. Dacal, M. M. de Lima, Jr., C. V. R. S. Ruffo, F. Iikawa, M. J. S. P. Brasil, T. Chiaramonte, M. A. Cotta, L. H. G. Tizei, D. Ugarte, and A. Cantarero, Phys. Rev. B 82, 125327 (2010).

${ }^{42}$ L. Zhang, J.-W. Luo, A. Zunger, N. Akopian, V. Zwiller, and J.-C. Harmand, Nanoletters 10, 4055 (2010).

${ }^{43}$ D. Li, Z. Wang, and F. Gao, Nanotechnology 21, 505709 (2010).

${ }^{44}$ L. C. O. Dacal and A. Cantarero, Solid State Commun. 151, 781 (2011).

${ }^{45}$ M. Moewe, L. C. Chuang, V. G. Dubrovskii, and C. Chang-Hasnain, J. Appl. Phys. 104, 044313 (2008).

${ }^{46}$ I. Vurgaftman, J. R. Meyer, and L. R. Ram-Mohan, J. Appl. Phys. 89, 5815 (2001).

${ }^{47}$ S. L. Chuang and C. S. Chang, Appl. Phys. Lett. 68, 1657 (1996).

${ }^{48}$ M. Holm, M. E. Pistol, and C. Pryor, J. Appl. Phys. 92, 932 (2002).

${ }^{49}$ See basic Indium Phosphide parameters $(300 \mathrm{~K})$ at the Ioffe Institute's New Semiconductor Materials electronic archive, http://www.ioffe.ru/ SVA/NSM/Semicond/InP/basic.html.

${ }^{50}$ Y. A. Bychkov and E. I. Rashba, J. Phys. C: Solid State Phys. 17, 6039 (1984).

${ }^{51}$ R. Winkler, Spin-Orbit Coupling Effects in Two-Dimensional Electron and Hole Systems (Springer, Berlin, 2003).

${ }^{52}$ See supplementary material at http://dx.doi.org/10.1063/1.4767511 for bulk InP WZ and ZB band structures, ZB strain determination, band structures of potentials in Fig. 6 and potentials that lead to the band structures of Fig. 9. 
Supplemental material for the paper "Band structure calculations of InP wurtzite/zinc-blende quantum wells"

P. E. Faria Junior ${ }^{1}$ and G. M. Sipahi ${ }^{1,2}$

1) Instituto de Física de São Carlos, Universidade de São Paulo, Caixa Postal 369, 13560-970 São Carlos, SP, Brazil

2) Department of Physics, State University of New York at Buffalo, Buffalo, New York 14260, USA 


\section{BULK CALCULATION - CHEKING PARAMETERS}

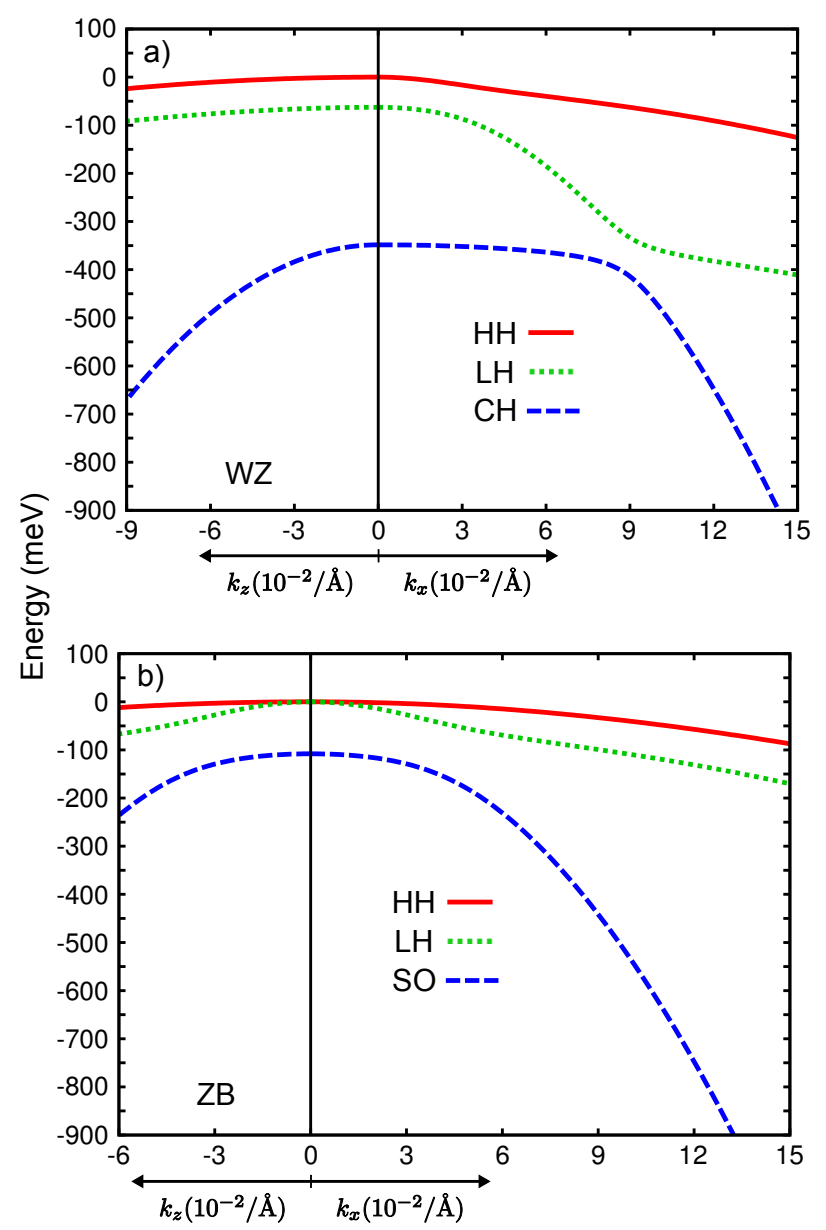

Figure 1. (Color online) Valence band structure for bulk (a) WZ and (b) ZB in the primed coordinate system. The usual identification of the bands was used for ZB while in WZ it was necessary to analyze the composition of the states in the $\Gamma$-point. We can see the anisotropy between $k_{z}$ and $k_{x}$ in WZ and also in ZB because in the new coordinate system the $x$ and $z$ axes do not reach equivalent points in the reciprocal space. The top of the valence band in both crystal structures was chosen to be at zero.

The resulting valence band structures for bulk WZ and ZB InP are shown in Figure 1, using Hamiltonian (5) of the article without strain effects. The presence of the crystal field in the WZ structure creates three distinct two-fold degenerate bands whereas in ZB there is a four-fold and a two-fold degenerate set of bands. Additionally, the anisotropy between $k_{x}$ and $k_{x}$ is evident in both crystal structures. In WZ it is due to the different symmetry 
properties of the $x y$-plane and the $z$ axis but in ZB it is because $k_{x}$ and $k_{z}$ directions (in the primed coordinate system of Fig. 4) do not reach equivalent points in the reciprocal space (Fig. 1 of Park and Chuang's paper ${ }^{1}$ ). Since the conduction band is a parabolic model, we did not present its dispersion relation.

Following Chuang and Chang ${ }^{2}$ notation, the valence energy bands for WZ are named after the composition of the states at $\vec{k}=0$. HH (heavy hole) states are composed only by $\left|c_{1}\right\rangle$ or $\left|c_{4}\right\rangle$, LH (light hole) states are composed mainly of $\left|c_{2}\right\rangle$ or $\left|c_{5}\right\rangle$ and $\mathrm{CH}$ (crystal-field split-off hole) are composed mainly of $\left|c_{3}\right\rangle$ or $\left|c_{6}\right\rangle$. For the ZB structure the common identification of the valence energy bands was used. The four-fold degenerate bands at $\vec{k}=0$ are $\mathrm{HH}$ and LH and the lower two-fold degenerate band is SO (split-off hole).

\section{STRAIN DETERMINATION}

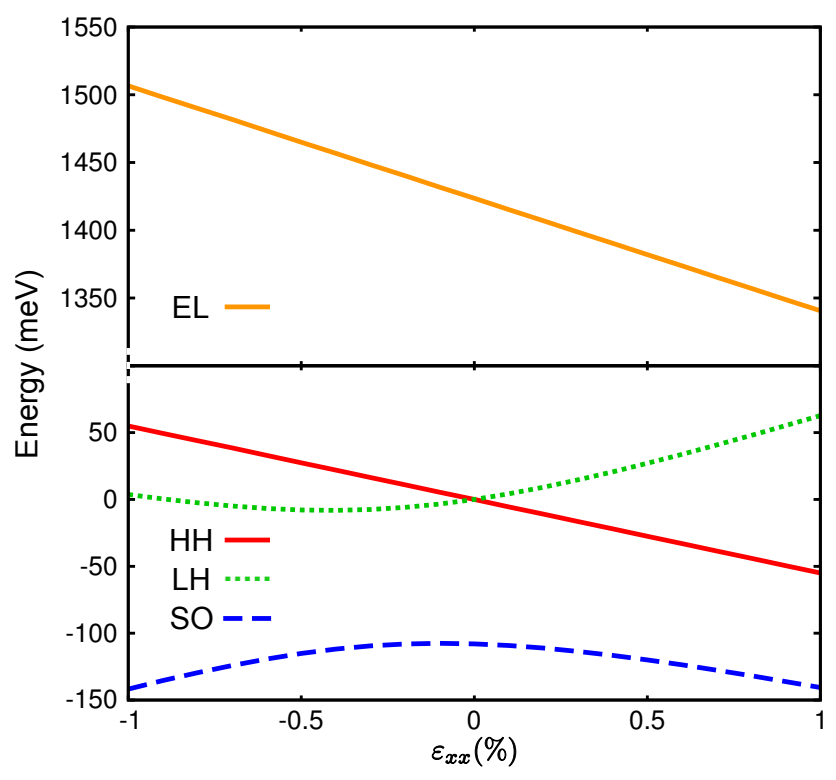

Figure 2. (Color online) Strain effect in the band edges of ZB InP as a function of the percentage of strain tensor. EL and HH shows a linear variation while LH and SO shows a nonlinear behavior. The strain effect removes the HH and LH degeneracy.

Figure 2 shows the effect of strain at $\vec{k}=0$ for the diagonalized Hamiltonian $(\mathrm{k} \cdot \mathrm{p}$ and strain terms) as a function of the percentage of strain tensor. A linear variation for the conduction band and the $\mathrm{HH}$ band is observed, however, the $\mathrm{LH}$ and SO bands have a 
non-linear behavior. The order of the $\mathrm{HH}$ and $\mathrm{LH}$ bands changes when strain is distensive. We can observe that for negative values of the strain tensor in the range of the values from ab initio calculations discuessed in the article, the LH is below the HH state. Hence, the physical properties derived from our calculations are consistent.

\section{BAND STRUCTURES OF WZ/ZB/WZ QUANTUM WELL WITH AND WITHOUT STRAIN EFFECTS}

The conduction and valence band structures of the WZ/ZB/WZ quantum well without strain effects are presented in Fig. 3 for three different widths of the ZB region. The calculations were performed up to $10 \%$ in the $\Gamma-T$ direction and $100 \%$ in the $\Gamma-A$ direction. For the valence band 64 energy states are presented while only 18 are presented for the conduction band. Since the system has no asymmetric potential, the energy bands

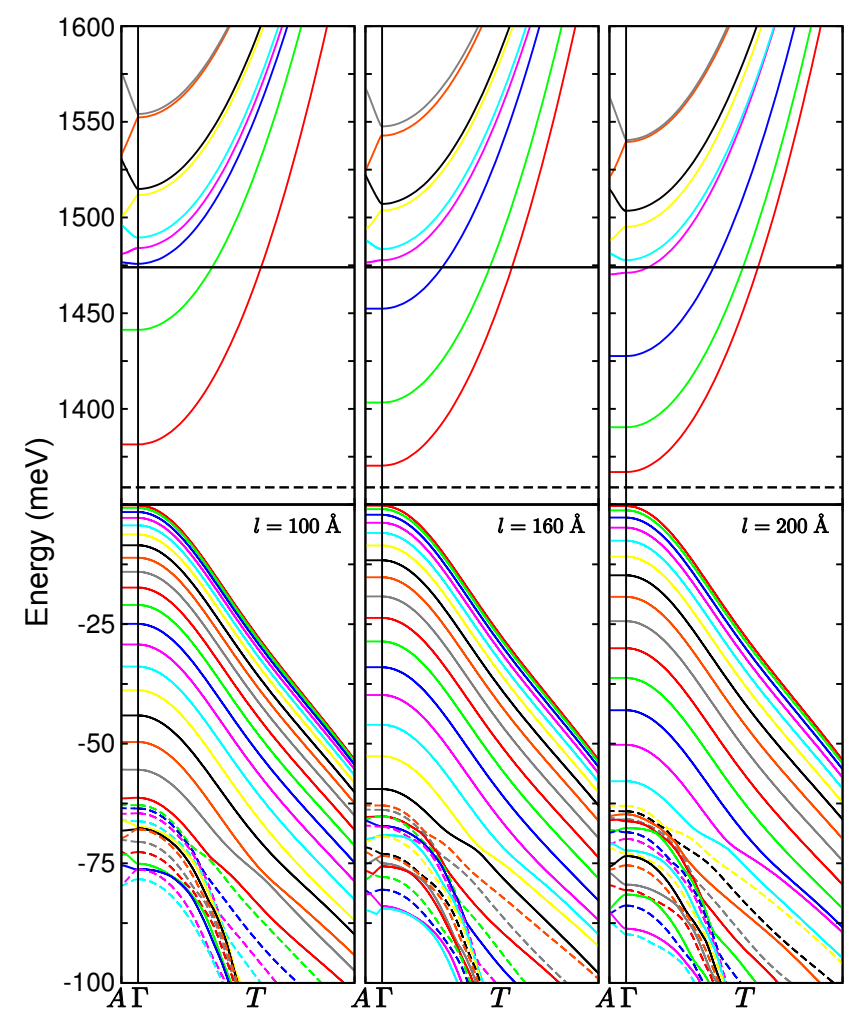

Figure 3. (Color online) Band structure without strain effects for the WZ/ZB/WZ polytypical quantum well. The solid horizontal line is the top energy of conduction band well and the dashed horizontal line is the bottom energy of the conduction band well. The $\Gamma-T$ direction refers to $k_{x}$ and $\Gamma-A$ to $k_{z}$. 
are two-fold degenerate in spin, therefore 32 states are visible in the valence band and 9 in the conduction band. For the three different values of $l$, the conduction energy bands are nominated, from bottom to top, as EL1-EL9, composed of $\left|c_{7,8}\right\rangle$ states. In the valence bands, solid lines are HH states and dashed lines are LH states.

Since the highest valence band states are HH there is no significant anticrossing among the energy bands in the $\Gamma-T$ direction. The anticrossing is characteristic of interactions between $\mathrm{HH}$ and LH bands in ZB and WZ quantum well structures. A slight anticrossing, however, can be seen in the energy region just above $-75 \mathrm{meV}$, which is next to the interaction region of the $\left|c_{1,4}\right\rangle$ and $\left|c_{2,5}\right\rangle$ profiles.

For the strained potential profile, the band structures for three different widths of the ZB region are displayed in Figure 4. The calculations were performed considering the same extension for the FBZ of the unstrained band structure. For the three different values of $l$, the conduction energy bands are nominated, from bottom to top, as EL1-EL9, composed of

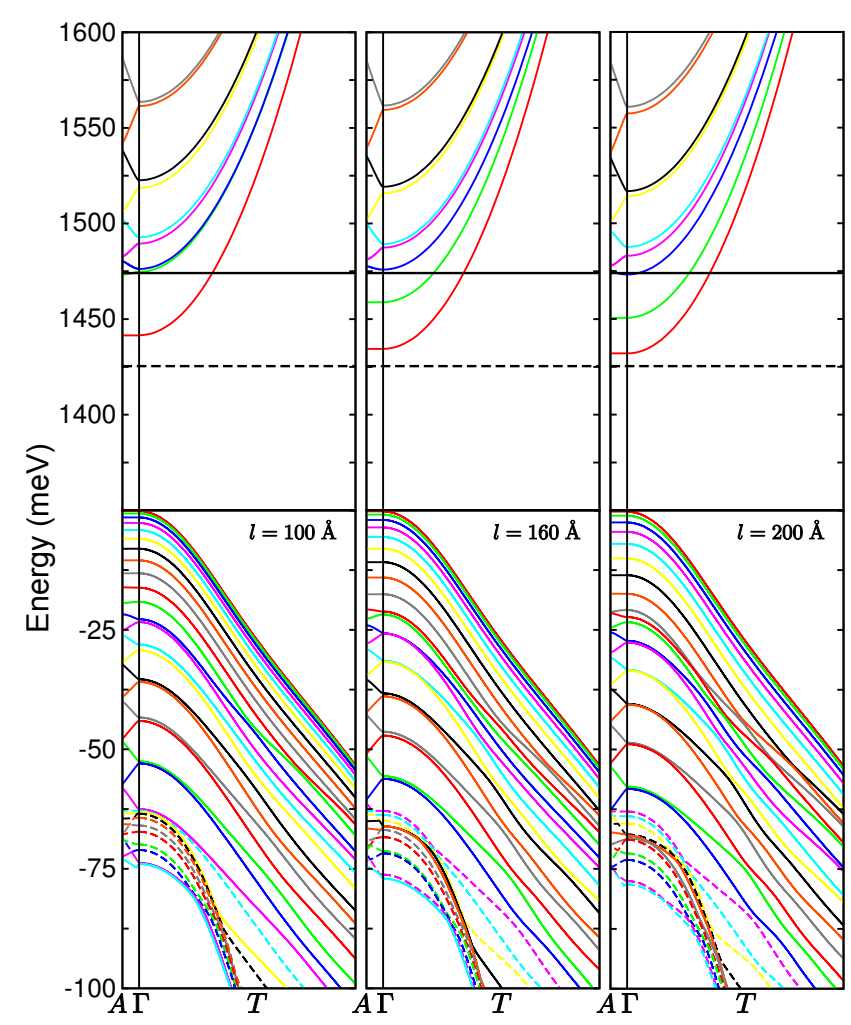

Figure 4. (Color online) Band structure with strain effects for the WZ/ZB/WZ polytypical quantum well. The solid and dashed lines have the same meaning as in Figure 3. The $\Gamma-T$ direction refers to $k_{x}$ and $\Gamma-A$ to $k_{z}$. 
$\left|c_{7,8}\right\rangle$ states. In the valence bands, solid lines are HH states and dashed lines are LH states.

An interesting feature presented in the strained band structure is the presence of some confined states below the top region, around $-62 \mathrm{meV}$. This suggests a confinement in the intermediate region of the $\left|c_{2,5}\right\rangle$ and $\left|c_{3,6}\right\rangle$ profiles. Note that the coupling of these two profiles at $\vec{k}=0$ happens because of the off-diagonal spin-orbit term.

\section{QUANTUM WELL PROFILES WITH POLARIZATION EFFECTS}

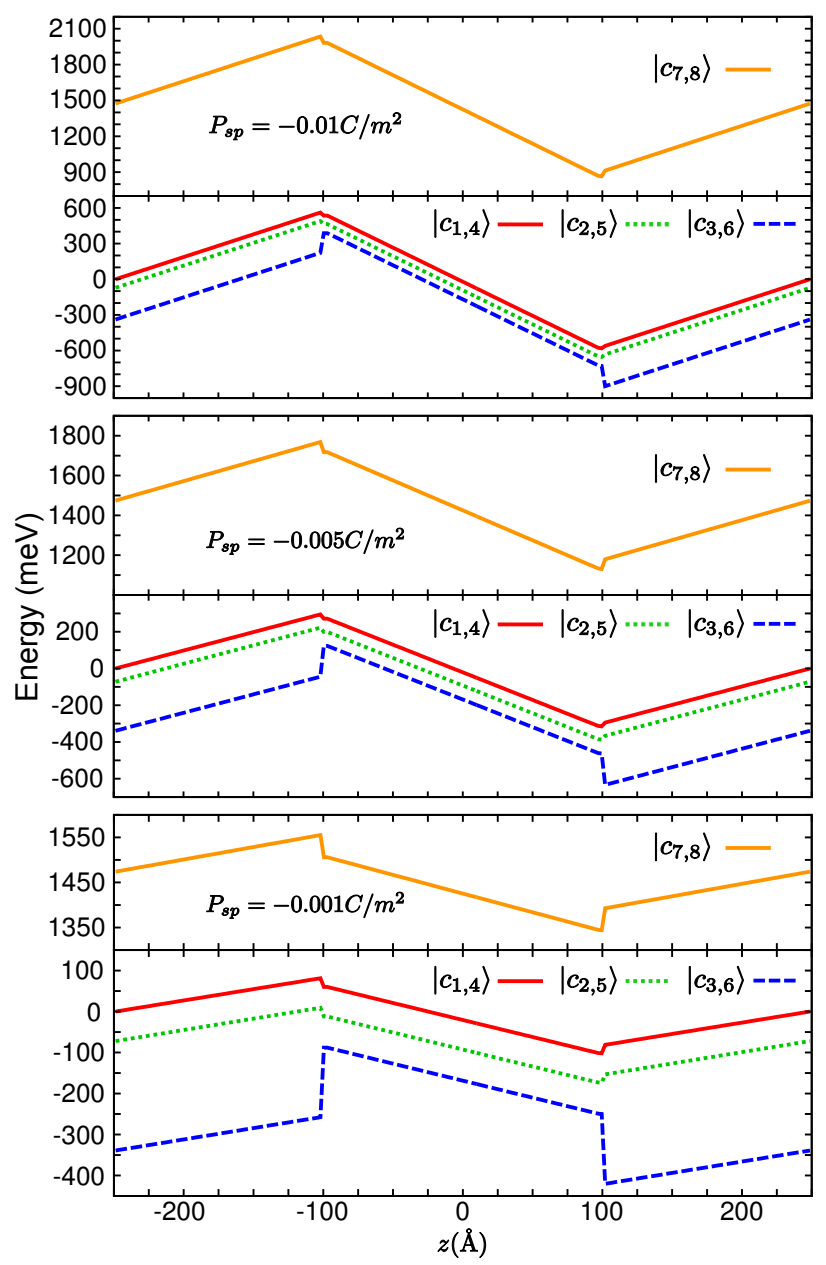

Figure 5. (Color online) Diagonal potential profile of the Hamiltonian for the polytypical InP system considering strain, piezoelectric polarization in the ZB region and spontaneous polarization in the WZ region for $l=200 \AA$. 
The diagonal potential profile including effects of piezoelectric and spontaneous polarization is presented in Figure 5. The ZB region is fixed at $200 \AA$. Analyzing these profiles, we expect to have strong coupling in the band structure for higher values of $P_{s p}$ since the profiles are closer to each other. This induces the mixing of states because an energy value can be in more than one profile.

\section{REFERENCES}

${ }^{1}$ S. H. Park and S. L. Chuang, Journal of Applied Physics 87, 353 (2000).

${ }^{2}$ S. L. Chuang and C. S. Chang, Applied Physics Letters 68, 1657 (1996). 


\title{
4.3 Interband polarized absorption in InP polytypic super- lattices
}

\author{
P. E. Faria Junior
}

Instituto de Física de São Carlos, Universidade de São Paulo, 13566-590 São Carlos, São Paulo, Brazil

Department of Physics, State University of New York at Buffalo, Buffalo, New York 14260, USA

\section{T. Campos}

Instituto de Física de São Carlos, Universidade de São Paulo, 13566-590 São Carlos, São Paulo, Brazil

\section{G. M. Sipahi}

Instituto de Física de São Carlos, Universidade de São Paulo, 13566-590 São Carlos, São Paulo, Brazil

Department of Physics, State University of New York at Buffalo, Buffalo, New York 14260, USA 


\title{
Interband polarized absorption in InP polytypic superlattices
}

\author{
P. E. Faria Junior, ${ }^{1,2}$ T. Campos, ${ }^{1}$ and G. M. Sipahi ${ }^{1,2}$ \\ ${ }^{1}$ Instituto de Física de São Carlos, Universidade de São Paulo, 13566-590 São Carlos, São Paulo, Brazil \\ ${ }^{2}$ Department of Physics, State University of New York at Buffalo, Buffalo, New York 14260, USA
}

(Received 25 September 2014; accepted 25 October 2014; published online 17 November 2014)

\begin{abstract}
Recent advances in growth techniques have allowed the fabrication of semiconductor nanostructures with mixed wurtzite/zinc-blende crystal phases. Although the optical characterization of these polytypic structures is well reported in the literature, a deeper theoretical understanding of how crystal phase mixing and quantum confinement change the output linear light polarization is still needed. In this paper, we theoretically investigate the mixing effects of wurtzite and zinc-blende phases on the interband absorption and in the degree of light polarization of an InP polytypic superlattice. We use a single $8 \times 8 \mathrm{k} \cdot \mathrm{p}$ Hamiltonian that describes both crystal phases. Quantum confinement is investigated by changing the size of the polytypic unit cell. We also include the optical confinement effect due to the dielectric mismatch between the superlattice and the vaccum and we show it to be necessary to match experimental results. Our calculations for large wurtzite concentrations and small quantum confinement explain the optical trends of recent photoluminescence excitation measurements. Furthermore, we find a high sensitivity to zinc-blende concentrations in the degree of linear polarization. This sensitivity can be reduced by increasing quantum confinement. In conclusion, our theoretical analysis provides an explanation for optical trends in InP polytypic superlattices, and shows that the interplay of crystal phase mixing and quantum confinement is an area worth exploring for light polarization engineering. (C) 2014 AIP Publishing LLC. [http://dx.doi.org/10.1063/1.4901209]
\end{abstract}

\section{INTRODUCTION}

The past few years has seen tremendous advances in growth techniques of low dimensional semiconductor nanostructures, especially concerning III-V nanowires (NWs). At the moment, it is possible to precisely tune the growth conditions to achieve single crystal phase nanostructures ${ }^{1-3}$ or polytypic heterostructures with sharp interfaces. ${ }^{4,5}$ Moreover, it has been reported successful integration of III-V NWs with silicon, ${ }^{6-9}$ increasing the possibilities for developing new optoelectronic devices. ${ }^{10,11}$

Because of its lower surface recombination and higher electron mobility, ${ }^{12,13} \mathrm{InP}$ is a good candidate, among the III-V compounds, to be embedded in these novel devices. Polytypic InP homojunctions showing a type-II band alignment ${ }^{14}$ can be explored to engineer light polarization ${ }^{15}$ and to enhance the lifetime of carriers. ${ }^{16,17}$ In fact, the use of InP NWs has been proposed in FETs, ${ }^{18-20}$ silicon integrated nanolasers, ${ }^{21}$ and stacked p-n junctions in solar cells. ${ }^{22,23}$

Although the process underlying the formation of these polytypic homojunctions is elucidated ${ }^{24-27}$ and an extensive literature on the optical characterization of these structures is available, ${ }^{9,28-32}$ we lack theoretical understanding of how the crystal phase mixture changes the light polarization on these nanostructures.

The aim of this study is to provide a comprehensive analysis on how wurtzite (WZ)/zinc-blende (ZB) mixing, quantum confinement (QC), and also optical confinement (OC) modify the interband absorption and the degree of linear polarization (DLP) in an InP polytypic superlattice. From now on, we will use the term superlattice for the polytypic case. In our calculations, the QC along growth direction takes into account the changes of $\mathrm{WZ}$ and $\mathrm{ZB}$ phases. Also, assuming large crosssections, we neglect lateral QC.
A scheme of the superlattice and possible light polarizations is presented in Fig. 1(a). Although we show a NW with multiple WZ and ZB segments, the periodicity of these segments allows us to consider only the unit cell, bounded by dashed lines, to understand the physics of the superlattice. The incoming light polarization can be either parallel $(\mathrm{Z})$ or perpendicular $(\mathrm{X})$ to the growth direction.
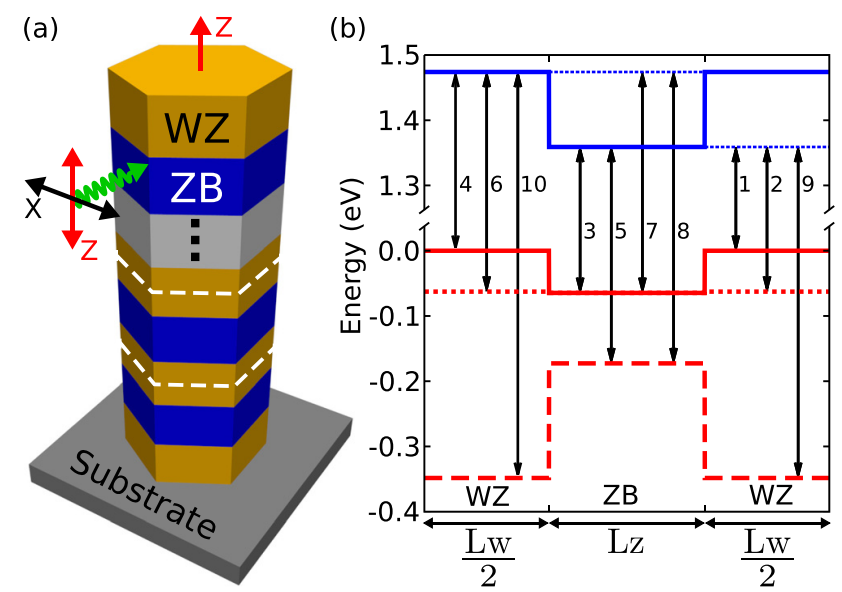

FIG. 1. (a) InP polytypic superlattice grown along WZ[0001]/ZB[111] direction. The (red) arrow on top indicates the growth direction. Polarization of incident light can be in X- or Z-direction, i.e., perpendicular or parallel to the growth direction. The polytypic superlattice unit cell, or simply supercell, of size $L=\mathrm{Lw}+\mathrm{Lz}$ is bounded by dashed (white) lines. (b) Band-edge energy diagram at $\vec{k}=0$ of type-II InP supercell with possible transitions. The numbers on the side of the vertical arrows indicate the magnitude of energy transitions, i.e., 1 means the lowest and 10 the highest. For the valence band, solid line is heavy hole band for both WZ/ZB, dotted line is light hole band for both $\mathrm{WZ} / \mathrm{ZB}$, and dashed line is crystal-field split-off hole band for WZ and splitoff hole band for ZB. The small dotted lines in the conduction band are plotted just to guide the eyes. 
To calculate the band structure, we extend our polytypic $\mathrm{k} \cdot \mathrm{p}$ method $^{33}$ and include the conduction and valence band interaction explicitly. This interaction increases the reliability of the method, and allows us to calculate the band structure further away from the center of the Brillouin zone. Furthermore, we provide the parameter sets for $\mathrm{WZ}$ and $\mathrm{ZB}$ InP in this new $8 \times 8 \mathrm{k} \cdot \mathrm{p}$ configuration.

We find that the trends of recent photoluminescence (PL) and excitation photoluminescence (PLE) measurements performed by Gadret et al. ${ }^{31}$ can be explained by our model. Although their samples are disordered, i.e., the regions of WZ/ZB are not periodically ordered, we can predict the observed trends considering a supercell of $100 \mathrm{~nm}$ composed of $95 \%$ WZ. In addition, we show that the DLP can be tuned using WZ/ZB mixing and QC. The limiting cases of our superlattice, i.e., pure ZB and pure WZ NWs are also calculated and their DLP around gap energy is in very good agreement with the results from Mishra et al. ${ }^{29}$ This matching emphasizes the use of OC in our calculations.

The structure of the present paper is the following: In Sec. II, we describe the $8 \times 8$ polytypic k.p method and present our approach for the interband transitions. Section III contains our results for interband absorption and DLP in the bulk and superlattice regimes. Finally, in Sec. IV, we summarize our main findings and present our conclusions.

\section{THEORETICAL BACKGROUND}

\section{A. Hamiltonian}

We expand the Hamiltonian of Ref. 33 to explicitly include the interband interaction. Since there is no coupling between the $\mathrm{ZB}$ irreducible representations for conduction $\left(\Gamma_{1} \sim x^{2}+y^{2}+z^{2}\right)$ and valence $\left(\Gamma_{15} \sim x, y, z\right)$ bands, we can apply the same rotation ${ }^{34}$ for the [001] $\mathrm{k} \cdot \mathrm{p}$ matrix with interband interaction. The total rotation matrix would be the direct sum of valence and conduction band rotation matrices, therefore an $8 \times 8$ matrix with $6 \times 6$ and $2 \times 2$ blocks. An alternative procedure would be to start with the Hamiltonian in the [111] coordinate system without interband interaction and derive the interband matrix elements in the [111] coordinate system relating them to the [001].

Our bulk Hamiltonian basis set, defined at $\Gamma$-point, in the $\mathrm{ZB}[111] / \mathrm{WZ}[0001]$ coordinate system is

$$
\begin{aligned}
& \left|c_{1}\right\rangle=-\frac{1}{\sqrt{2}}|(X+i Y) \uparrow\rangle \\
& \left|c_{2}\right\rangle=\frac{1}{\sqrt{2}}|(X-i Y) \uparrow\rangle \\
& \left|c_{3}\right\rangle=|Z \uparrow\rangle \\
& \left|c_{4}\right\rangle=\frac{1}{\sqrt{2}}|(X-i Y) \downarrow\rangle \\
& \left|c_{5}\right\rangle=-\frac{1}{\sqrt{2}}|(X+i Y) \downarrow\rangle \\
& \left|c_{6}\right\rangle=|Z \downarrow\rangle \\
& \left|c_{7}\right\rangle=i|S \uparrow\rangle \\
& \left|c_{8}\right\rangle=i|S \downarrow\rangle
\end{aligned}
$$

with 1-6 representing the valence band states and 7-8 the conduction band states. In this basis set, the Hamiltonian including interband interactions is given by

$$
\mathbb{H}_{8}=\left[\begin{array}{cc}
\mathbb{H}_{\mathrm{V}} & \mathbb{H}_{\mathrm{VC}} \\
\mathbb{H}_{\mathrm{VC}}^{\dagger} & \mathbb{H}_{\mathrm{C}}
\end{array}\right],
$$

where $\mathbb{H}_{\mathrm{V}}$ represent the valence band, $\mathbb{H}_{\mathrm{C}}$ is the conduction band, and $\mathbb{H}_{\mathrm{VC}}$ is the interaction term between them. The sub-matrices have the following forms:

$$
\mathbb{H}_{\mathrm{V}}=\left[\begin{array}{cccccc}
F & -K^{*} & -H^{*} & 0 & 0 & 0 \\
-K & G & H & 0 & 0 & \sqrt{2} \Delta_{3} \\
-H & H^{*} & \lambda & 0 & \sqrt{2} \Delta_{3} & 0 \\
0 & 0 & 0 & F & -K & H \\
0 & 0 & \sqrt{2} \Delta_{3} & -K^{*} & G & -H^{*} \\
0 & \sqrt{2} \Delta_{3} & 0 & H^{*} & -H & \lambda
\end{array}\right]
$$

$$
\begin{array}{r}
\mathbb{H}_{\mathrm{VC}}=\left[\begin{array}{cc}
-\frac{1}{\sqrt{2}} P_{2} k_{-} & 0 \\
\frac{1}{\sqrt{2}} P_{2} k_{+} & 0 \\
P_{1} k_{z} & 0 \\
0 & \frac{1}{\sqrt{2}} P_{2} k_{+} \\
0 & -\frac{1}{\sqrt{2}} P_{2} k_{-} \\
0 & P_{1} k_{z}
\end{array}\right], \\
\mathbb{H}_{\mathrm{C}}=\left[\begin{array}{cc}
E_{C} & 0 \\
0 & E_{C}
\end{array}\right]
\end{array}
$$

and their terms

$$
\begin{aligned}
F & =\Delta_{1}+\Delta_{2}+\lambda+\theta \\
G & =\Delta_{1}-\Delta_{2}+\lambda+\theta \\
\lambda & =\tilde{A}_{1} k_{z}^{2}+\tilde{A}_{2}\left(k_{x}^{2}+k_{y}^{2}\right), \\
\theta & =\tilde{A}_{3} k_{z}^{2}+\tilde{A}_{4}\left(k_{x}^{2}+k_{y}^{2}\right), \\
K & =\tilde{A}_{5} k_{+}^{2}+2 \sqrt{2} \tilde{A}_{z} k_{-} k_{z}, \\
H & =\tilde{A}_{6} k_{+} k_{z}+\tilde{A}_{z} k_{-}^{2}, \\
E_{C} & =E_{g}+E_{0}+\tilde{e}_{1} k_{z}^{2}+\tilde{e}_{2}\left(k_{x}^{2}+k_{y}^{2}\right),
\end{aligned}
$$

where $\tilde{A}_{1} \ldots \tilde{A}_{6}, \tilde{A}_{z}$ and $\tilde{e}_{1}, \tilde{e}_{2}$, given in units of $\hbar^{2} / 2 m_{0}$, are the effective mass parameters of valence and conduction bands, respectively. Here $\Delta_{1}$ is the crystal field splitting energy in WZ, $\Delta_{2}, \Delta_{3}$ are the spin-orbit coupling splitting energies, $k_{ \pm}=k_{x} \pm i k_{y}$ and $P_{1}, P_{2}$ are the Kane parameters of the interband interactions, given by 


$$
\begin{aligned}
P_{1} & =-i \frac{\hbar}{m_{0}}\left\langle X\left|p_{x}\right| S\right\rangle=-i \frac{\hbar}{m_{0}}\left\langle Y\left|p_{y}\right| S\right\rangle, \\
P_{2} & =-i \frac{\hbar}{m_{0}}\left\langle Z\left|p_{z}\right| S\right\rangle .
\end{aligned}
$$

We would like to emphasize that the Hamiltonian (2) and its terms (6) describe both WZ and ZB crystal structures, however, the usual $\mathrm{ZB}$ parameters must be mapped to the ones in Eq. (6). Moreover, the inclusion of the interband interaction explicitly in the Hamiltonian also requires some corrections to be made in the second order effective mass parameters. These corrections appear because conduction and valence band states are now treated as belonging to the same class, following Löwdin's notation. ${ }^{35}$ We describe the mapping and corrections of parameters with detail in the Appendix.

In order to treat the confined direction along $z$, we apply the envelope function approximation ${ }^{36,37}$ to the Hamiltonian (2). This treatment can be summarized with the following changes:

$$
\begin{aligned}
g & \rightarrow g(z) \\
k_{z} & \rightarrow-i \frac{\partial}{\partial z} \\
B k_{z}^{2} & \rightarrow-\frac{\partial}{\partial z} B(z) \frac{\partial}{\partial z} \\
P k_{z} & \rightarrow-\frac{i}{2}\left[\frac{\partial}{\partial z} P(z)+P(z) \frac{\partial}{\partial z}\right]
\end{aligned}
$$

with $g$ representing the parameters in the Hamiltonian (different in $\mathrm{WZ}$ and $\mathrm{ZB}$ ), $B$ representing an effective mass parameter and $P$ is the interband parameter. The last two equations are the symmetrization requirements to hold the Hermitian property of the momentum operator. ${ }^{37}$ Any parameter in the Hamiltonian acquires a dependence along $z$, making it different for $\mathrm{WZ}$ and $\mathrm{ZB}$. Also, the confinement profile due to the polytypic interface is added to the Hamiltonian. In Fig. 1(b), we present the InP band-edge profile along $z$ for $\vec{k}=0$, which takes into account the interface profile and the intrinsic splittings of each crystal structure.

Under the envelope function approximation, a general state $n, \vec{k}$ of the superlattice can be written as

$$
\psi_{n, \vec{k}}(\vec{r})=e^{i \vec{k} \cdot \vec{r}} \sum_{m=1}^{8} f_{n, \vec{k}, m}(z) u_{m}(\vec{r}) .
$$

We then apply the plane wave expansion for the parameters and the envelope functions to solve the Hamiltonian numerically. Since this expansion automatically considers periodic boundary conditions, we can associate the value $k_{z}$ for the superlattice, $-\frac{\pi}{L} \leq k_{z} \leq \frac{\pi}{L}$, while the Fourier coefficients have $K_{j}=j \frac{2 \pi}{L}$ (with $j=0, \pm 1, \pm 2, \ldots$ ). For confined states, the dispersion of the band structure along $k_{z}$ is a flat band. However, higher energy states are no longer confined and does not have this flat dispersion. Since we are interested in transitions that also take into account these higher energy states, we will include the $k_{z}$ in our calculation.

\section{B. Interband absorption}

The absorption coefficient ${ }^{38}$ of photons with energy $\hbar \omega$ can be written as

$$
\alpha_{\hat{\epsilon}}(\hbar \omega)=\frac{C}{\hbar \omega} \sum_{a, b, \vec{k}} I_{a, b, \vec{k}}^{\hat{\epsilon}}\left(f_{a, \vec{k}}-f_{b, \vec{k}}\right) \mathcal{L}_{a, b, \vec{k}}^{\Gamma}(\hbar \omega),
$$

where $a(b)$ runs over conduction (valence) sub-bands, $\vec{k}$ runs over reciprocal space points, $\hat{\epsilon}$ is the light polarization, $I_{a, b, \vec{k}}^{\hat{e}}$ is the interband dipole transition amplitude, $\mathcal{L}_{a, b, \vec{k}}^{\Gamma}(\hbar \omega)$ gives the transition broadening, and $f_{a(b), \vec{k}}$ is the Fermi-Dirac distribution of conduction (valence) band. We will consider $T=0 \mathrm{~K}$ and no doping, i.e., the valence band is full and the conduction band is empty, ${ }^{39}$ leading to $f_{a, \vec{k}}-f_{b, \vec{k}}=1$. The constant $C$ is given by

$$
C=\frac{\pi \hbar e^{2}}{c n_{r} \varepsilon_{0} m_{0}^{2} \Omega}
$$

where $e$ is the electron charge, $c$ is the velocity of light, $n_{r}$ is the refractive index of the material, $\epsilon_{0}$ is the vacuum dielectric constant, $m_{0}$ is the free electron mass, and $\Omega$ is the volume of the real space.

We considered a Lorentzian broadening for the transitions

$$
\mathcal{L}_{a, b, \vec{k}}^{\Gamma}(\hbar \omega)=\frac{1}{2 \pi} \frac{\Gamma}{\left[E_{a}(\vec{k})-E_{b}(\vec{k})-\hbar \omega\right]^{2}+\left(\frac{\Gamma}{2}\right)^{2}}
$$

with $\Gamma$ as the full width at half-maximum. In our calculations, we set $\Gamma=2 \mathrm{meV}$.

For $\hat{x}$ and $\hat{z}$ light polarizations, the interband dipole transition amplitudes, between conduction $(a)$ and valence $(b)$ states, are given by

$$
\begin{aligned}
I_{a, b, \vec{k}}^{\hat{x}} \propto & \frac{1}{2} \mid\left\langle F_{a, \vec{k}, 7} \mid F_{b, \vec{k}, 1}\right\rangle+\left\langle F_{a, \vec{k}, 1} \mid F_{b, \vec{k}, 7}\right\rangle \\
& -\left\langle F_{a, \vec{k}, 7} \mid F_{b, \vec{k}, 2}\right\rangle-\left\langle F_{a, \vec{k}, 2} \mid F_{b, \vec{k}, 7}\right\rangle \\
& -\left\langle F_{a, \vec{k}, 8} \mid F_{b, \vec{k}, 4}\right\rangle-\left\langle F_{a, \vec{k}, 4} \mid F_{b, \vec{k}, 8}\right\rangle \\
& +\left\langle F_{a, \vec{k}, 8} \mid F_{b, \vec{k}, 5}\right\rangle+\left.\left\langle F_{a, \vec{k}, 5} \mid F_{b, \vec{k}, 8}\right\rangle\right|^{2}, \\
I_{a, b, \vec{k}}^{\hat{z}} \propto & \mid\left\langle F_{a, \vec{k}, 7} \mid F_{b, \vec{k}, 3}\right\rangle+\left\langle F_{a, \vec{k}, 3} \mid F_{b, \vec{k}, 7}\right\rangle \\
& +\left\langle F_{a, \vec{k}, 8} \mid F_{b, \vec{k}, 6}\right\rangle+\left.\left\langle F_{a, \vec{k}, 6} \mid F_{b, \vec{k}, 8}\right\rangle\right|^{2}
\end{aligned}
$$

with

$$
\left\langle F_{a, \vec{k}, m} \mid F_{b, \vec{k}, n}\right\rangle=\frac{1}{L} \int_{L} d z f_{a, \vec{k}, m}^{*}(z) f_{b, \vec{k}, n}(z),
$$

where $L$ is the size of the supercell and the factor $1 / L$ appears because our envelope functions are normalized in reciprocal space.

We assumed the interband coupling parameters to be constant throughout the polytypic system, i.e., the same values were used for both polytypes since their numerical values do not differ much (see Table I).

The relative contributions of the different light polarizations can be probed by analyzing the DLP: 
TABLE I. k p parameters of the polytypic $8 \times 8$ model for InP.

\begin{tabular}{lcr}
\hline \hline Parameter & ZB InP & WZ InP \\
\hline Lattice constant $(\AA)$ & & \\
$a$ & 4.1505 & 4.1505 \\
$c$ & 10.1666 & 6.7777 \\
Energy parameters (eV) & & \\
$E_{g}$ & 1.4236 & 1.474 \\
$\Delta_{1}$ & 0 & 0.303 \\
$\Delta_{2}=\Delta_{3}$ & 0.036 & 0.036 \\
Conduction band effective mass & & \\
parameters (units of $\left.\frac{\hbar^{2}}{2 m_{0}}\right)$ & & \\
$\tilde{e}_{1}$ & -1.6202 & -1.2486 \\
$\tilde{e}_{2}$ & -1.6202 & -1.6231 \\
Valence band effective mass & & \\
parameters (units of $\left.\frac{\hbar^{2}}{2 m_{0}}\right)$ & & -0.8299 \\
$\tilde{A}_{1}$ & 1.0605 & -0.8423 \\
$\tilde{A}_{2}$ & -0.8799 & 1.2001 \\
$\tilde{A}_{3}$ & -1.9404 & 0.8272 \\
$\tilde{A}_{4}$ & 0.9702 & 0.3902 \\
$\tilde{A}_{5}$ & 1.4702 & \\
$\tilde{A}_{6}$ & 2.7863 & \\
$\tilde{A}_{z}$ & -0.5000 & \\
Interband coupling parameters $(\mathrm{eV} \AA)$ & & \\
$P_{1}=P_{2}$ & & \\
\hline \hline
\end{tabular}

$$
\operatorname{DLP}(\hbar \omega)=\frac{\alpha_{\hat{z}}(\hbar \omega)-\alpha_{\hat{x}}(\hbar \omega)}{\alpha_{\hat{z}}(\hbar \omega)+\alpha_{\hat{x}}(\hbar \omega)}
$$

which ranges from -1 , if the absorbed light is polarized perpendicular to the wire axis, to 1 , if polarization is parallel to the growth direction.

We have also investigated the effect of $\mathrm{OC}$ due to the dielectric mismatch between the vacuum and the superlattice. This effect is included as follows: ${ }^{40}$

$$
\alpha_{\hat{x}}(\hbar \omega) \rightarrow \frac{2}{1+\epsilon} \alpha_{\hat{x}}(\hbar \omega)
$$

with $\epsilon$ being the dielectric constant of the superlattice, which was considered to be the same for ZB and WZ InP $(\epsilon=12.4$ (Refs. 41 and 42)).

\section{RESULTS AND DISCUSSION}

\section{A. Bulk}

Before we turn to the superlattice case, it is useful to understand the light polarization properties for bulk $\mathrm{ZB}$ and WZ. Indeed, these would be the limiting cases of our superlattice calculations. We can view these bulk limiting cases as NWs of large diameter and length, with pure crystal phases. Here, we also assumed the light polarizations described in Fig. 1(a). Moreover, the $8 \times 8$ parameter sets were derived from the $6 \times 6$ model of our previous paper, ${ }^{33}$ which was based on the effective masses of Ref. 43. The lattice constants $a$ and $c$ of ZB are given in the [111] unit cell (ZB has three bilayers of atoms instead of two in WZ). Table I have all the parameters used in the simulations.

In Fig. 2(a), we show the band structure of bulk $\mathrm{ZB}[111]$ and WZ[0001] for $k_{x}$ and $k_{z}$ directions. At $\vec{k}=0$,
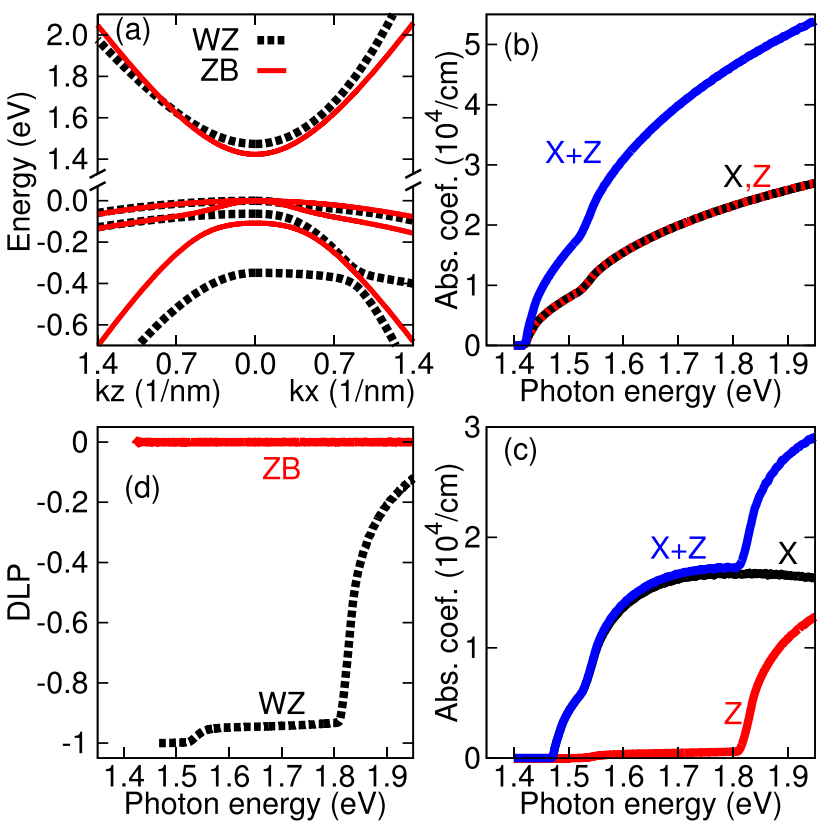

FIG. 2. In clockwise order: (a) InP bulk band structure for WZ (dashed lines) and ZB (solid lines). InP Bulk ZB (b) and WZ (c) absorption coefficients as functions of the photon energies. (d) $\mathrm{ZB}$ (solid line) and WZ (dashed line) DLP. The crystal structure plays an important role in the absorption: ZB absorbs light isotropically, while WZ have a clear anisotropy that changes when photon energy reaches the band edge energies.

the valence band of WZ has three energy bands two-fold degenerate, while $\mathrm{ZB}$ has one four-fold degenerate band and one two-fold degenerate. From top to bottom, WZ valence bands are labeled HH (heavy hole), LH (light hole), and $\mathrm{CH}$ (crystal-field split-off hole), following Chuang and Chang's notation, ${ }^{44}$ and $\mathrm{ZB}$ valence bands are labeled usually as $\mathrm{HH} /$ LH and SO (split-off hole). Each band-edge in the band structure will have a signature in the absorption spectra; therefore, we can expect three regions for $\mathrm{WZ}$ and only two for ZB. Moreover, the symmetry of the eigenstates will rule the light polarization, as shown by Eqs. (13) and (14).

Figures 2(b) and 2(c) show the bulk absorption coefficients for $\mathrm{ZB}$ and $\mathrm{WZ}$, respectively, as calculated by Eq. (10). Although we considered ZB in the [111] unit cell, $\mathrm{X}$ - and Z-polarizations have the same absorption as we would expect from the cubic symmetry. One can easily show that the coordinate system rotation we have applied holds this cubic character in the absorption coefficient. Note the shoulder in the curve when the photon energy reaches the SO band energy. For WZ, however, a clear anisotropy between X- and Z-polarizations is found. Before we reach the $\mathrm{CH}$ band energy, light is predominantly $\mathrm{X}$-polarized, however, after $\mathrm{CH}$ band the $\mathrm{Z}$-polarized absorption increases while X-polarized slightly decreases.

To highlight the polarization differences for $\mathrm{ZB}$ and WZ, we show in Fig. 2(d) the DLP, given by Eq. (16). Since $\mathrm{X}$ - and Z-polarizations are the same in $\mathrm{ZB}$, we have a straight line at DLP $=0$, meaning isotropic absorption. For WZ, the DLP starts at -1 , slightly increases when LH band is reached and after $\mathrm{CH}$ band, it rapidly goes to 0 due to the Z-polarization contribution. In the superlattice calculations, we expect that the WZ/ZB mixing and QC effects will 
change the DLP to some intermediate value between pure $\mathrm{ZB}$ and $\mathrm{WZ}$.

\section{B. Absorption and PLE measurements}

Let us start the superlattice investigation by considering small QC, i.e., relatively large $\mathrm{WZ}$ and ZB regions (5-90 nm each with total supercell of $100 \mathrm{~nm}$ ), values typically found in superlattice samples. Although there is small QC, small regions of $\mathrm{WZ}$ and $\mathrm{ZB}$ act as perturbations to the bulk states leading to different electronic and optical properties.

In Fig. 3(a), we show the total absorption, $\alpha_{\hat{x}}+\alpha_{\hat{z}}$, without OC effects. The first (top) solid curve is the case of $90 \%$ of ZB and $10 \%$ of WZ and we can notice the two characteristic shoulders of the bulk case, the first around transition energy 3 and the second around transition 5. Increasing the mixing of $\mathrm{ZB}$ and $\mathrm{WZ}$, new shoulders appear and when we reach the last (bottom) solid curve, $90 \%$ of $\mathrm{WZ}$ and $10 \%$ of $\mathrm{ZB}$, we notice the three characteristic shoulders of WZ bulk case, around transitions 4, 6, and 10, respectively. This WZ characteristic is also noted in the dashed line, which is the 95\%WZ/5\%ZB. We can also notice from Fig. 3(a) that there is a non-zero absorption coefficient between $\mathrm{ZB}$ and $\mathrm{WZ}$ energy gaps (transitions 2 and 4) even for large WZ concentrations. This phenomenon is due to the characteristic type-II band alignment of $\mathrm{ZB} / \mathrm{WZ}$ homojunctions.

When we add OC effects, which are presented in Fig. 3(b), we notice a significant suppression of X-polarization that becomes more evident as WZ/ZB ratio increases. Since

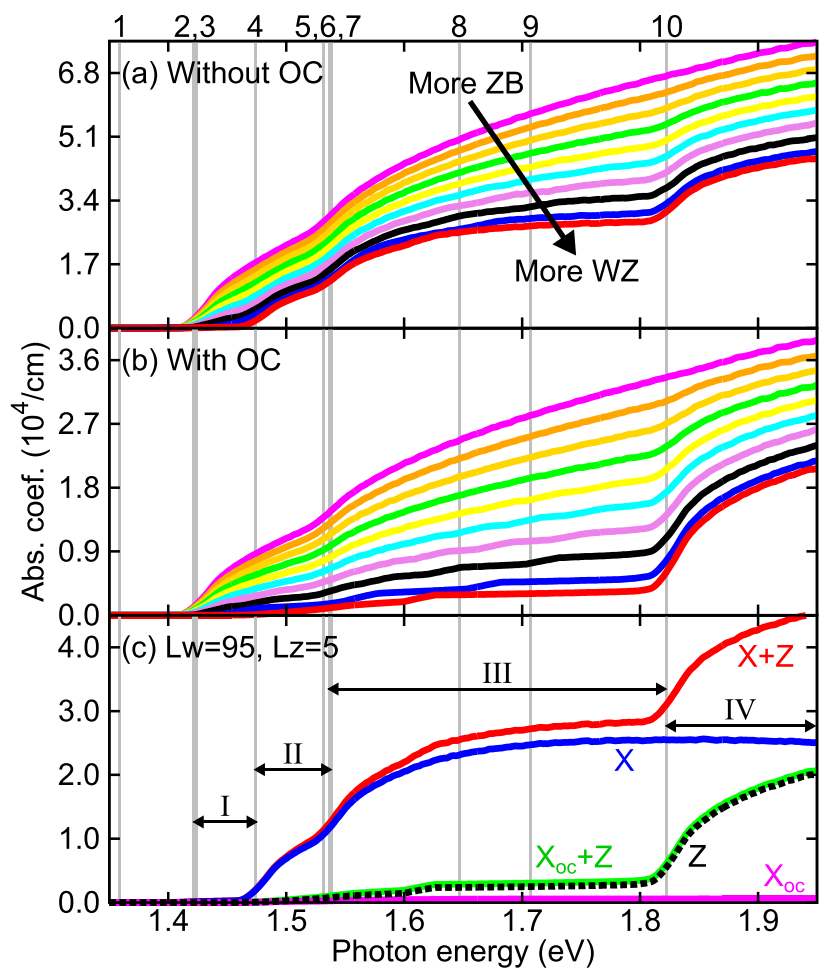

FIG. 3. Absorption coefficient for $100 \mathrm{~nm}$ supercell with different WZ/ZB ratio (a) without $\mathrm{OC}$ and (b) with OC. From top to bottom, solid lines range from $10 \% \mathrm{WZ} / 90 \% \mathrm{ZB}$ to $90 \% \mathrm{WZ} / 10 \% \mathrm{ZB}$ with steps of $10 \%$. The dashed line is the $95 \% \mathrm{WZ} / 5 \% \mathrm{ZB}$ regime. (c) Absorption coefficient for $95 \% \mathrm{WZ} /$ $5 \% \mathrm{ZB}$, including the contributions for $\mathrm{X}$ - and $\mathrm{Z}$-polarizations. The numbers at the top of the figure indicate the transition energies of Fig. 1(b). in WZ, the absorption spectrum in regions I and II is mainly comprises sub-bands with a mixture of states $\left|c_{1}\right\rangle,\left|c_{4}\right\rangle$ due to $\mathrm{HH}$ symmetry, the $\mathrm{OC}$ almost forbids the $\mathrm{X}$ component from penetrating the NW, therefore the suppression.

The experimental paper of Gadret et al. ${ }^{31}$ investigates optical properties of InP polytypic superlattices. They report PL and PLE measurements of InP polytypic samples with statistically negligible percentage of $\mathrm{ZB}$. In this regime, they notice three absorption edges in the PLE $(\sim 1.488 \mathrm{eV}$, $\sim 1.532 \mathrm{eV}$, and $\sim 1.675 \mathrm{eV}$ ) for energies above the PL peak $(\sim 1.432 \mathrm{eV})$ and also a long tail at the low energy side of the PL peak. Their system is comparable to our simulation for $100 \mathrm{~nm}$ supercell with $95 \%$ of $\mathrm{WZ}$ and $5 \%$ of $\mathrm{ZB}$ or even higher WZ percentage over ZB. Indeed, the measured trends are well described by our Fig. 3(c). We can identify four regions that we can relate to the experimental spectra: I (between transitions $2 / 3$ and 4, i.e., between $\mathrm{ZB}$ and $\mathrm{WZ}$ gap energies), II (between transitions 4 and 5/6/7), III (between transitions 5/6/7 and 10), and IV (after transition 10). From the observed data, we can assign the three absorption edges to the beginning of energy regions II, III, and IV, respectively. Region I actually comprises the region where the PL peak is observed. Furthermore, the long tail at low energy side of PL can be explained by the type-II confinement of WZ/ZB interface, which has negligible absorption coefficient. Since we are not considering excitons, we do not observe the peaks in the absorption at the band-edge transitions (visible in the experimental data). However, the bandedge character is well described by our model, represented by the shoulders in our graphs. The blue-shift of our bandedge transition energies compared to the experimental data is also related to the lack of excitonic effects in our model.

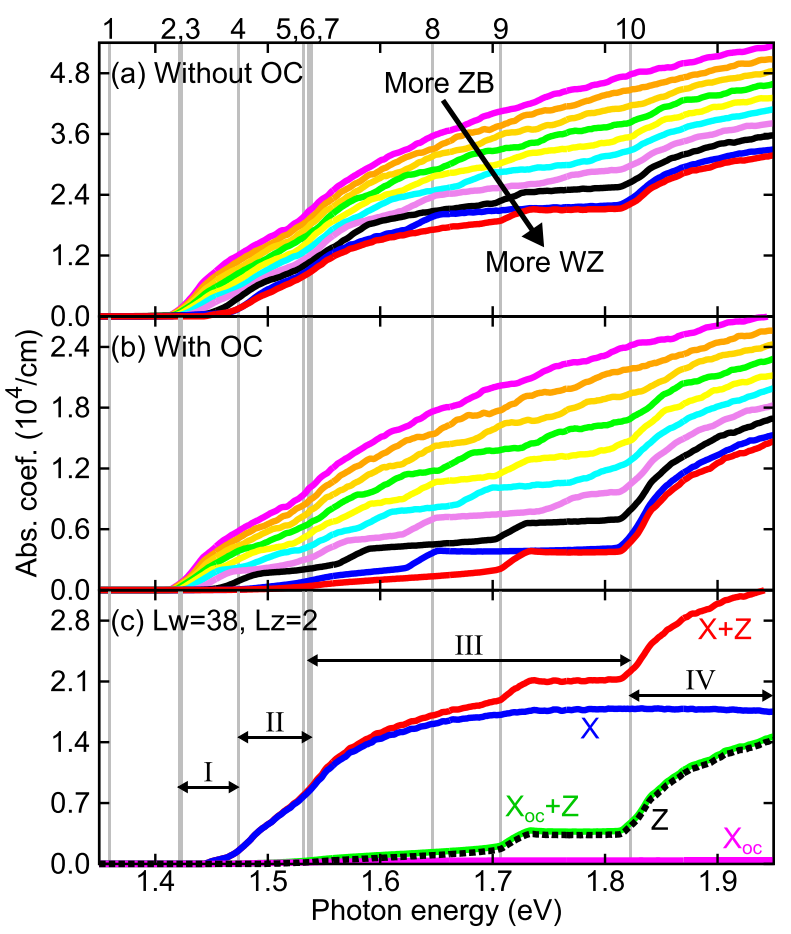

FIG. 4. Same as system Fig. 3 for $40 \mathrm{~nm}$ supercell. In this case, because of the smaller supercell, QC effects are more pronounced. 


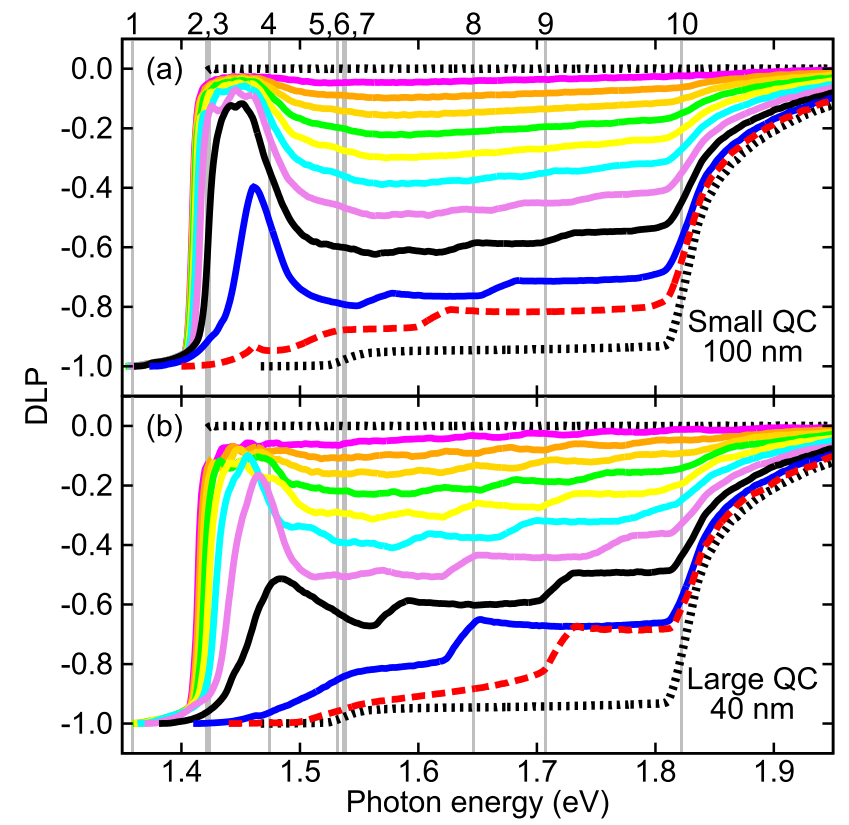

FIG. 5. Degree of linear polarization without optical confinement for small QC (a) and large QC (b). The straight dotted line at 0 indicates the bulk ZB limit, whereas the lowest dotted line is the bulk WZ limit. The solid and dashed lines have the same meaning as in Figs. 3 and 4.

For comparison, we plotted in Fig. 4 the same results but for a $40 \mathrm{~nm}$ supercell. In Figs. 4(a) and 4(b), we observe the same trends as before but with more quantization effects, signalled by the extra shoulders or step-like behavior in absorption spectra. As we increase the QC, the number of sub-bands in the same energy range decreases, leading to clear shoulders in the spectra as the photon energy reaches these few sub-bands. In Fig. 4(c), we show the different contribution of X- and Z-polarizations. Comparing to Fig. 3(c), it can be seen that the QC effect is more visible in Z-polarization since this is the confined direction. For the X-polarization, a small red-shift is observed due to greater overlap between states.

\section{Quantum confinement and crystal phase mixing effects in the DLP}

For a better understanding of the optical properties of the InP homojunctions, it is valuable to study the DLP. Specifically, we are interested in how polarization properties are modified by different crystal phase mixing and QC.

In Fig. 5, we show the behavior of the DLP only under QC effects, no OC included, for the case in which the supercell has 100 nm, Fig. 5(a), and 40 nm, Fig. 5(b). In general, the DLP is very sensitive to ZB insertions: In region $\mathrm{I}$, it is close to 0 , exception made to systems where $\mathrm{WZ}$ regions are largely dominant over $\mathrm{ZB}$ ones, about $80 \% \mathrm{WZ}$ or more for the small QC regime and $70 \% \mathrm{WZ}$ or more in large QC. For all different WZ/ZB mixing, the limits are bulk WZ and bulk ZB DLP, presented in Fig. 2(d) and showed here with dotted lines.

To further analyze the effect of the OC, we present in Fig. 6 the DLP calculations for the same systems previously discussed, including QC and OC effects. Here, we also include OC effects in bulk calculations. In the paper of

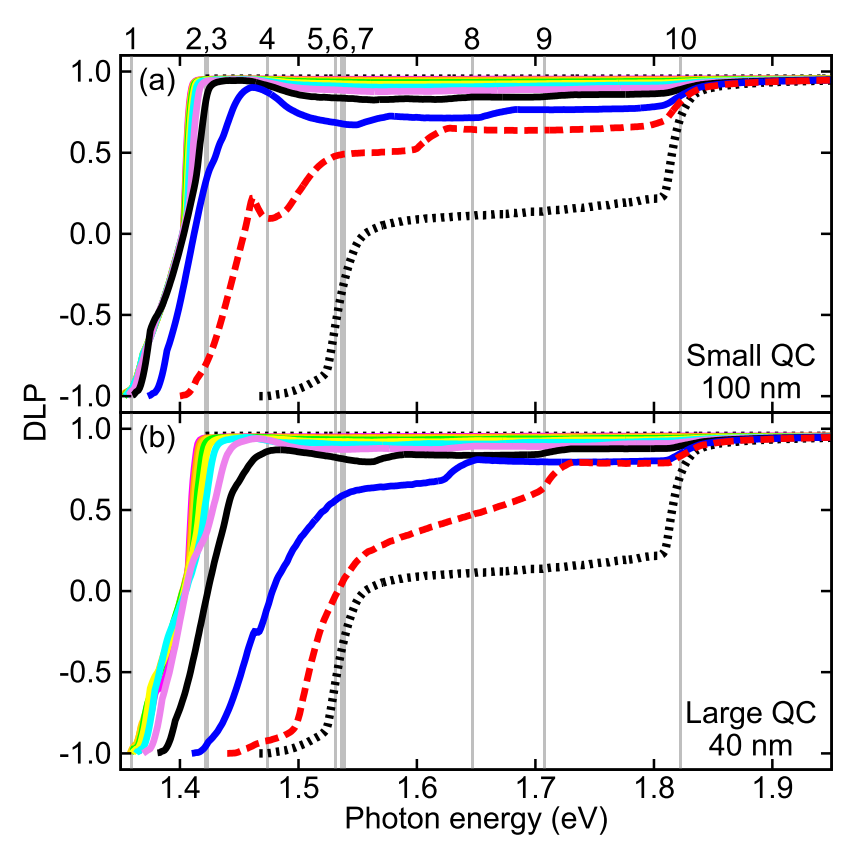

FIG. 6. Same as Fig. 5 but including optical confinement. The straight dotted line at 1 indicates the bulk $\mathrm{ZB}$ limit, whereas the lowest dotted line is the bulk WZ limit.

Mishra et al., ${ }^{29}$ their PL measurements for pure WZ and ZB NWs with large diameters indicate that for ZB light is strongly polarized along the NW axis, whereas for WZ light is strongly polarized in the perpendicular direction. Our calculations for the bulk case with OC are in very good agreement with these experimental results. In fact, this indicates that $\mathrm{OC}$ is a necessary feature to be included in the description. Also, these are the limiting cases for all WZ/ZB mixed systems.

Comparing the results with OC for small QC, Fig. 6(a), and large QC, Fig. 6(b), we also notice that the DLP is very susceptible to $\mathrm{ZB}$ concentration, i.e., just a small amount of ZB can switch the DLP from -1 to approximately 1 . Moreover, for large QC, WZ features hold more effectively around the gap energy. This happens because WZ holes (parallel to growth direction) have larger effective masses $\left(m_{H H}^{\mathrm{ZB}}=0.532\right.$ and $\left.m_{H H \|}^{\mathrm{WZ}}=1.273\right)$ and, therefore, are the dominant symmetry to light polarization. Hence, increasing QC can reduce the ZB susceptibility to the DLP.

As a final remark, if we compare our DLP for small QC to the PL spectra measurement of Gadret et al., ${ }^{31}$ we also notice a non-zero value for parallel polarization. Since their NWs have a statistically negligible percentage of $\mathrm{ZB}$ and still present parallel polarization, we believe that this corroborates our results of ZB susceptibility to the DLP.

\section{CONCLUSIONS}

We have expanded our previous polytypic $\mathrm{k} \cdot \mathrm{p}$ model $^{33}$ to include interband coupling explicitly. The validation of our $8 \times 8$ model was considered for the bulk case, and we found how selection rules for $\mathrm{WZ}$ and $\mathrm{ZB}$ allow different light polarization features.

For the InP polytypic superlattice, we found good agreement between our results and the experimental 
measurements of PLE and light polarization. Although we have not considered excitonic effects, the energy regions from the paper of Gadret et al. ${ }^{31}$ can be mapped to our calculations with small QC and large WZ composition. When QC is increased, step like features are observed in the interband absorption for Z-polarization, which is parallel to the growth direction.

Since WZ and ZB present different optical selection rules, any mixing of these two crystal phases should combine these different light polarizations. Our DLP calculations for pure $\mathrm{ZB}$ or pure $\mathrm{WZ}$ are in good agreement with experiments of Mishra et al., ${ }^{29}$ and OC effects were necessary to match the experimental results. Stronger QC retains the WZ behavior of the DLP, since WZ HH has larger effective mass than $\mathrm{ZB} \mathrm{HH}$. In the polytypic cases, we found that the DLP is very susceptible to $\mathrm{ZB}$ regions and only a small amount of ZB drastically increases the DLP. This ZB susceptibility is increased if QC effects are decreased. Furthermore, our results for the DLP also explain the polarized PL measured by Gadret et al. ${ }^{31}$

In summary, we believe that our findings provide a theoretical explanation for the optical properties observed in InP polytypic superlattices and also indicate how linear light polarization can be tuned using QC and crystal phase mixing. We wish to emphasize that our theoretical approach is not only limited to InP but also could be applied to other III$\mathrm{V}$ compounds that exhibit polytypism. ${ }^{43}$

\section{ACKNOWLEDGMENTS}

The authors acknowledge financial support from the Brazilian agencies CNPq (Grant Nos. 138457/2011-5, 246549/ 2012-2, and 149904/2013-4) and FAPESP (Grant Nos. 2011/ 19333-4, 2012/05618-0, and 2013/23393-8). The authors thank James P. Parry and Karie Friedman for kindly proofreading the paper.

\section{APPENDIX: SECOND-ORDER CORRECTIONS}

When the interband coupling is considered in $\mathrm{k} \cdot \mathrm{p}$ Hamiltonians, it is necessary to correct some of the second order parameters due to the modification of states that belong to classes $\mathrm{A}$ and $\mathrm{B} .{ }^{35}$ For the Luttinger parameters in $\mathrm{ZB}$, we have

$$
\begin{aligned}
\tilde{\gamma}_{1} & =\gamma_{1}-\frac{E_{P}}{3 E_{g}} \\
\tilde{\gamma}_{2} & =\gamma_{2}-\frac{E_{P}}{6 E_{g}} \\
\tilde{\gamma}_{3} & =\gamma_{3}-\frac{E_{P}}{6 E_{g}} \\
F & =\frac{1}{m_{e}^{*}}-\frac{E_{g}+\frac{2}{3} \Delta_{S O}}{E_{g}+\Delta_{S O}} \frac{E_{P}}{E_{g}} \\
E_{P} & =\frac{2 m_{0}}{\hbar^{2}} P^{2}
\end{aligned}
$$

and for WZ parameters, we have

$$
\begin{aligned}
\tilde{A}_{1} & =A_{1}+\frac{E_{P 1}}{E_{g}+\Delta_{1}} \\
\tilde{A}_{2} & =A_{2} \\
\tilde{A}_{3} & =A_{3}-\frac{E_{P 1}}{E_{g}+\Delta_{1}} \\
\tilde{A}_{4} & =A_{4}+\frac{1}{2} \frac{E_{P 2}}{E_{g}} \\
\tilde{A}_{5} & =A_{5}+\frac{1}{2} \frac{E_{P 2}}{E_{g}} \\
\tilde{A}_{6} & =A_{6}+\frac{1}{\sqrt{2}} \frac{\sqrt{E_{P 1} E_{P 2}}}{E_{g}+\frac{\Delta_{1}}{2}} \\
\tilde{e}_{1} & =e_{1}-\frac{E_{P 1}}{E_{g}+\Delta_{1}} \\
\tilde{e}_{2} & =e_{2}-\frac{E_{P 2}}{E_{g}} \\
E_{P 1(2)} & =\frac{2 m_{0}}{\hbar^{2}} P_{1(2)}^{2} .
\end{aligned}
$$

Given the corrected Luttinger parameters, we only have to connect them to the ones in the Hamiltonian (2), using the same idea as presented in our previous paper ${ }^{33}$

$$
\begin{aligned}
& \Delta_{1}=0 \\
& \Delta_{2}=\Delta_{3}=\frac{\Delta_{S O}}{3} \\
& \tilde{A}_{1}=-\tilde{\gamma}_{1}-4 \tilde{\gamma}_{3} \\
& \tilde{A}_{2}=-\tilde{\gamma}_{1}+2 \tilde{\gamma}_{3} \\
& \tilde{A}_{3}=6 \tilde{\gamma}_{3} \\
& \tilde{A}_{4}=-3 \tilde{\gamma}_{3} \\
& \tilde{A}_{5}=-\tilde{\gamma}_{2}-2 \tilde{\gamma}_{3} \\
& \tilde{A}_{6}=-\sqrt{2}\left(2 \tilde{\gamma}_{2}+\tilde{\gamma}_{3}\right) \\
& \tilde{A}_{z}=\tilde{\gamma}_{2}-\tilde{\gamma}_{3} \\
& \tilde{e}_{1}=\tilde{e}_{2}=F \\
& P_{1}=P_{2}=P
\end{aligned}
$$

For the numerical values presented in Table I, we first corrected the $\mathrm{ZB}$ parameters and then applied the connection to the polytypic Hamiltonian.

${ }^{1}$ P. Mohan, J. Motohisa, and T. Fukui, Nanotechnology 16, 2903 (2005).

${ }^{2}$ T. T. T. Vu, T. Zehender, M. A. Verheijen, S. R. Plissard, G. W. G. Immink, J. E. M. Haverkort, and E. P. A. M. Bakkers, Nanotechnology 24, 115705 (2013).

${ }^{3}$ D. Pan, M. Fu, X. Yu, X. Wang, L. Zhu, S. Nie, S. Wang, Q. Chen, P. Xiong, S. von Molnár, and J. Zhao, Nano Lett. 14, 1214 (2014).

${ }^{4}$ S. Lehmann, J. Wallentin, D. Jacobsson, K. Deppert, and K. A. Dick, Nano Lett. 13, 4099 (2013).

${ }^{5}$ V. Khranovskyy, A. M. Glushenkov, Y. Chen, A. Khalid, H. Zhang, L. Hultman, B. Monemar, and R. Yakimova, Nanotechnology 24, 215202 (2013).

${ }^{6}$ F. Ren, K. Wei Ng, K. Li, H. Sun, and C. J. Chang-Hasnain, Appl. Phys. Lett. 102, 012115 (2013).

${ }^{7}$ M. Borg, H. Schmid, K. E. Moselund, G. Signorello, L. Gignac, J. Bruley, C. Breslin, P. Das Kanungo, P. Werner, and H. Riel, Nano Lett. 14, 1914 (2014). ${ }^{8}$ M. Heiss, E. Russo-Averchi, A. Dalmau-Mallorquí, G. Tütüncüoğlu, F. Matteini, D. Rüffer, S. Conesa-Boj, O. Demichel, E. Alarcon-Lladó, A. Fontcuberta, and I. Morral, Nanotechnology 25, 014015 (2014). 
${ }^{9}$ K. Li, H. Sun, F. Ren, K. W. Ng, T.-T. D. Tran, R. Chen, and C. J. ChangHasnain, Nano Lett. 14, 183 (2014).

${ }^{10}$ N. Akopian, G. Patriarche, L. Liu, J.-C. Harmand, and V. Zwiller, Nano Lett. 10, 1198 (2010).

${ }^{11}$ M. Smit, X. Leijtens, H. Ambrosius, E. Bente, J. van der Tol, B. Smalbrugge, T. de Vries, E.-J. Geluk, J. Bolk, R. van Veldhoven, L. Augustin, P. Thijs, D. D'Agostino, H. Rabbani, K. Lawniczuk, S. Stopinski, S. Tahvili, A. Corradi, E. Kleijn, D. Dzibrou, M. Felicetti, E. Bitincka, V. Moskalenko, J. Zhao, R. Santos, G. Gilardi, W. Yao, K. Williams, P. Stabile, P. Kuindersma, J. Pello, S. Bhat, Y. Jiao, D. Heiss, G. Roelkens, M. Wale, P. Firth, F. Soares, N. Grote, M. Schell, H. Debregeas, M. Achouche, J.-L. Gentner, A. Bakker, T. Korthorst, D. Gallagher, A. Dabbs, A. Melloni, F. Morichetti, D. Melati, A. Wonfor, R. Penty, R. Broeke, B. Musk, and D. Robbins, Semicond. Sci. Technol. 29, 083001 (2014)

${ }^{12}$ H. J. Joyce, C. J. Docherty, Q. Gao, H. H. Tan, C. Jagadish, J. LloydHughes, L. M. Herz, and M. B. Johnston, Nanotechnology 24, 214006 (2013).

${ }^{13}$ C. S. Ponseca, H. Němec, J. Wallentin, N. Anttu, J. P. Beech, A. Iqbal, M. Borgström, M.-E. Pistol, L. Samuelson, and A. Yartsev, Phys. Rev. B 90, 085405 (2014).

${ }^{14}$ M. Murayama and T. Nakayama, Phys. Rev. B 49, 4710 (1994).

${ }^{15}$ T. Ba Hoang, A. F. Moses, L. Ahtapodov, H. Zhou, D. L. Dheeraj, A. T. J. van Helvoort, B.-O. Fimland, and H. Weman, Nano Lett. 10, 2927 (2010).

${ }^{16}$ K. Pemasiri, M. Montazeri, R. Gass, L. M. Smith, H. E. Jackson, J. Yarrison-Rice, S. Paiman, Q. Gao, H. H. Tan, C. Jagadish, X. Zhang, and J. Zou, Nano Lett. 9, 648 (2009).

${ }^{17}$ C. K. Yong, J. Wong-Leung, H. J. Joyce, J. Lloyd-Hughes, Q. Gao, H. H. Tan, C. Jagadish, M. B. Johnston, and L. M. Herz, Nano Lett. 13, 4280 (2013).

${ }^{18}$ X. Duan, Y. Huang, Y. Cui, J. Wang, and C. M. Lieber, Nature 409, 66 (2001).

${ }^{19}$ X. Jiang, Q. Xiong, S. Nam, F. Qian, Y. Li, and C. M. Lieber, Nano Lett. 7, 3214 (2007).

${ }^{20}$ J. Wallentin, M. Ek, L. R. Wallenberg, L. Samuelson, and M. T. Borgström, Nano Lett. 12, 151 (2012).

${ }^{21}$ Z. Wang, B. Tian, M. Paladugu, M. Pantouvaki, N. Le Thomas, C. Merckling, W. Guo, J. Dekoster, J. Van Campenhout, P. Absil, and D. Van Thourhout, Nano Lett. 13, 5063 (2013).

${ }^{22}$ J. Wallentin, N. Anttu, D. Asoli, M. Huffman, I. Aberg, M. H. Magnusson, G. Siefer, P. Fuss-Kailuweit, F. Dimroth, B. Witzigmann,
H. Q. Xu, L. Samuelson, K. Deppert, and M. T. Borgström, Science 339, 1057 (2013).

${ }^{23}$ Y. Cui, J. Wang, S. R. Plissard, A. Cavalli, T. T. T. Vu, R. P. J. van Veldhoven, L. Gao, M. Trainor, M. A. Verheijen, J. E. M. Haverkort, and E. P. A. M. Bakkers, Nano Lett. 13, 4113 (2013).

${ }^{24}$ T. Akiyama, K. Nakamura, and T. Ito, Phys. Rev. B 73, 235308 (2006).

${ }^{25}$ Y. Kitauchi, Y. Kobayashi, K. Tomioka, S. Hara, K. Hiruma, T. Fukui, and J. Motohisa, Nano Lett. 10, 1699 (2010).

${ }^{26}$ K. Ikejiri, Y. Kitauchi, K. Tomioka, J. Motohisa, and T. Fukui, Nano Lett. 11, 4314 (2011).

${ }^{27}$ P. J. Poole, D. Dalacu, X. Wu, J. Lapointe, and K. Mnaymneh, Nanotechnology 23, 385205 (2012).

${ }^{28}$ M. Mattila, T. Hakkarainen, M. Mulot, and H. Lipsanen, Nanotechnology 17, 1580 (2006).

${ }^{29}$ A. Mishra, L. V. Titova, T. B. Hoang, H. E. Jackson, L. M. Smith, J. M. Yarrison-Rice, Y. Kim, H. J. Joyce, Q. Gao, H. H. Tan, and C. Jagadish, Appl. Phys. Lett. 91, 263104 (2007).

${ }^{30}$ S. Paiman, Q. Gao, H. H. Tan, C. Jagadish, K. Pemasiri, M. Montazeri, H. E. Jackson, L. M. Smith, J. M. Yarrison-Rice, X. Zhang, and J. Zou, Nanotechnology 20, 225606 (2009).

${ }^{31}$ E. G. Gadret, G. O. Dias, L. C. O. Dacal, M. M. de Lima, C. V. R. S. Ruffo, F. Iikawa, M. J. S. P. Brasil, T. Chiaramonte, M. A. Cotta, L. H. G. Tizei, D. Ugarte, and A. Cantarero, Phys. Rev. B 82, 125327 (2010).

${ }^{32}$ P. Kailuweit, M. Peters, J. Leene, K. Mergenthaler, F. Dimroth, and A. W. Bett, Prog. Photovoltaics: Res. Appl. 20, 945 (2012).

${ }^{33}$ P. E. Faria Junior and G. M. Sipahi, J. Appl. Phys. 112, 103716 (2012).

${ }^{34}$ S. H. Park and S. L. Chuang, J. Appl. Phys. 87, 353 (2000).

${ }^{35}$ P. Löwdin, J. Chem. Phys. 19, 1396 (1951).

${ }^{36}$ G. Bastard, Phys. Rev. B 24, 5693 (1981).

${ }^{37}$ G. Baraff and D. Gershoni, Phys. Rev. B 43, 4011 (1991).

${ }^{38}$ S. L. Chuang, Physics of Optoelectronic Devices (John Wiley, New York, 1995).

${ }^{39}$ V. V. Ravi Kishore, B. Partoens, and F. M. Peeters, Phys. Rev. B 82, 235425 (2010).

${ }^{40}$ M. Califano and A. Zunger, Phys. Rev. B 70, 165317 (2004).

${ }^{41}$ J. Wang, M. S. Gudiksen, X. Duan, Y. Cui, and C. M. Lieber, Science 293, 1455 (2001)

${ }^{42}$ M. P. Persson and H. Q. Xu, Phys. Rev. B 70, 161310 (2004).

${ }^{43}$ A. De and C. E. Pryor, Phys. Rev. B 81, 155210 (2010).

${ }^{44}$ S. L. Chuang and C. S. Chang, Appl. Phys. Lett. 68, 1657 (1996). 


\title{
4.4 Realistic multiband $k \cdot p$ approach from $a b$ initio and spin- orbit coupling effects of InAs and InP in wurtzite phase
}

\author{
$\underline{\text { Paulo E. Faria Junior }}$
}

São Carlos Institute of Physics, University of São Paulo, 13566-590 São Carlos, São Paulo, Brazil

\section{Tiago Campos}

São Carlos Institute of Physics, University of São Paulo, 13566-590 São Carlos, São Paulo, Brazil Institute for Theoretical Physics, University of Regensburg, 93040 Regensburg, Germany

Carlos M. O. Bastos

São Carlos Institute of Physics, University of São Paulo, 13566-590 São Carlos, São Paulo, Brazil

\section{Martin Gmitra}

Institute for Theoretical Physics, University of Regensburg, 93040 Regensburg, Germany

Jaroslav Fabian

Institute for Theoretical Physics, University of Regensburg, 93040 Regensburg, Germany

\section{Guilherme M. Sipahi}

Instituto de Física de São Carlos, Universidade de São Paulo, 13566-590 São Carlos, São Paulo, Brazil Department of Physics, University at Buffalo, State University of New York, Buffalo, New York 14260, USA 


\title{
Realistic multiband $k \cdot p$ approach from $a b$ initio and spin-orbit coupling effects of InAs and InP in wurtzite phase
}

\author{
Paulo E. Faria Junior, ${ }^{1, *}$ Tiago Campos, ${ }^{1,2}$ Carlos M. O. Bastos, ${ }^{1}$ Martin Gmitra, ${ }^{2}$ Jaroslav Fabian, ${ }^{2}$ and Guilherme M. Sipahi ${ }^{1,3}$ \\ ${ }^{1}$ São Carlos Institute of Physics, University of São Paulo, 13566-590 São Carlos, São Paulo, Brazil \\ ${ }^{2}$ Institute for Theoretical Physics, University of Regensburg, 93040 Regensburg, Germany \\ ${ }^{3}$ Department of Physics, State University of New York at Buffalo, Buffalo, New York 14260, USA
}

(Received 20 April 2016; published 30 June 2016)

\begin{abstract}
Semiconductor nanowires based on non-nitride III-V compounds can be synthesized under certain growth conditions to favor the appearance of the wurtzite crystal phase. Despite reports in the literature of ab initio band structures for these wurtzite compounds, we still lack effective multiband models and parameter sets that can be simply used to investigate physical properties of such systems, for instance, under quantum confinement effects. In order to address this deficiency, in this study we calculate the $a b$ initio band structure of bulk InAs and InP in the wurtzite phase and develop an $8 \times 8 k \cdot p$ Hamiltonian to describe the energy bands around the $\Gamma$ point. We show that our $k \cdot p$ model is robust and can be fitted to describe the important features of the ab initio band structure. The correct description of the spin-splitting effects that arise due to the lack of inversion symmetry in wurtzite crystals is obtained with the $k$-dependent spin-orbit term in the Hamiltonian, often neglected in the literature. All the energy bands display a Rashba-like spin texture for the in-plane spin expectation value. We also provide the density of states and the carrier density as functions of the Fermi energy. Alternatively, we show an analytical description of the conduction band, valid close to the $\Gamma$ point. The same fitting procedure is applied to the $6 \times 6$ valence band Hamiltonian. However, we find that the most reliable approach is the $8 \times 8$ $k \cdot p$ Hamiltonian for both compounds. The $k \cdot p$ Hamiltonians and parameter sets that we develop in this paper provide a reliable theoretical framework that can be easily applied to investigate electronic, transport, optical, and spin properties of InAs- and InP-based nanostructures.
\end{abstract}

DOI: 10.1103/PhysRevB.93.235204

\section{INTRODUCTION}

In the past decade, the development of low-dimensional III-V semiconductor nanostructures has witnessed great advances [1]. For instance, one interesting feature that was observed in the synthesis of III-V nanowires is the appearance of the wurtzite (WZ) crystal phase, instead of the usual zinc-blende (ZB) [2]. This created new possibilities of III-V compounds with WZ structure besides the well-established nitride-based materials. Moreover, controlling the growth conditions, e.g., temperature and III/V ratio, it is possible to achieve not only single crystal phase nanowires [3,4] but also to mix $\mathrm{ZB}$ and $\mathrm{WZ}$ regions with sharp interfaces in the same nanostructure, which is known as polytypism [5-7]. It has been demonstrated that mixed phases greatly affect the physical properties, for example, of the light polarization [8-10], electron transport $[11,12]$, and photoconductivity [13].

Among these new III-V compounds with WZ structure, InAs and InP are particularly important. InAs WZ has a large spin-orbit coupling (SOC) which favors the study of spin-related phenomena, for instance, spin field effect transistors [14], and the search for the elusive Majorana fermions [15]. Also, InAs WZ shows remarkable piezoelectric and piezoresistive properties [16] that, combined with the InAs narrow band gap, can operate in the near-infrared regime. On the other hand, $\mathrm{InP}$ is a promising candidate for photovoltaic applications [17] and for enhancing the efficiency of solar cells [18]. In fact, a silicon-integrated nanolaser

*fariajunior.pe@gmail.com of InP nanowire has already been demonstrated at room temperature with a wide wavelength range due to the WZ/ZB polytypism [19]. Furthermore, it is also possible to combine InAs and InP WZ compounds in axial [20] and radial [21] heterostructures, which opens the path for novel opportunities in band gap engineering.

Theoretical studies based on WZ III-V compounds including InAs and InP were reported using different $a b$ initio approaches. The main focus of these studies was the determination of the lattice parameters, band gaps, and SOC energy splittings in the valence band [22-27]. Of these references, De and Pryor [23] provide useful information that can be used in effective models, such as the effective masses and the spin-splitting parameters. The issue of using these parameters is that they are only valid in the immediate vicinity of the $\Gamma$ point $[\sim 2 \%$ of the first Brillouin zone (FBZ)], limiting the range of physical phenomena that can be investigated. In order to achieve a better description farther away from the $\Gamma$ point, a multiband effective model is desirable. Although $k \cdot p$ models and parameters are well established for WZ III-nitride compounds $[28,29]$, there are only a few reports in the literature for non-nitrides, such as InP [10,30] and GaAs [31].

In this study, we develop a robust $8 \times 8 k \cdot p$ Hamiltonian to describe the $a b$ initio band structure calculated by WIEN2k [32] of InAs and InP in the WZ phase. We show that our fitted parameters reproduce the $a b$ initio band structure, capturing the important anticrossings and spinsplitting features up to $1.0 \mathrm{~nm}^{-1}\left(\sim 10 \%\right.$ of the FBZ in the $k_{x} k_{y}$ plane and $\sim 22 \%$ in the $k_{z}$ direction). At $\Gamma$ point, each band is twofold degenerate and for the valence band we found that the band ordering, from top to bottom, is $\mathrm{HH}$ (heavy hole), $\mathrm{CH}$ 
(crystal field split-off hole), and LH (light hole) for InAs and $\mathrm{HH}, \mathrm{LH}$, and $\mathrm{CH}$ for InP. This ordering is due to an interplay of SOC energy splittings and the crystal field energy splitting. The intricate behavior of spin splittings, arising from the bulk inversion asymmetry (BIA) of the WZ structure, is correctly described by the $k$-dependent SOC term, often neglected in the literature. Calculating the spin expectation value for the Bloch states, we find a Rashba-like spin texture [33] with either clockwise $(\mathrm{CW})$ or counterclockwise $(\mathrm{CCW})$ orientation. All these spin-dependent features extracted from our $k \cdot p$ Hamiltonian and parameter sets were systematically checked to agree with $a b$ initio calculations. Furthermore, based on our effective $8 \times 8$ Hamiltonian, we calculated the density of states (DOS) and predict the carrier density as a function of the Fermi energy. We also provide an analytical description of the conduction band valid close to the $\Gamma$ point and a compact description of the valence band $(6 \times 6$ Hamiltonian $)$. But, we would like to emphasize that the best description of InAs and InP WZ is obtained using the total $8 \times 8$ Hamiltonian. In summary, the main goal of our paper is to provide a realistic $k \cdot p$ description that can be used to study charge and spin transport, optics, as well as (superconducting) proximity effects in semiconductor heterostructures, e.g., quantum wells and wires. Such heterostructures cannot be investigated by first principles due to their mesoscopic sizes, and the $k \cdot p$ technique (using the prescription $\vec{k} \rightarrow-i \vec{\nabla}$ ) is currently perhaps the best choice for obtaining physically relevant quantities for them.

This paper is organized as follows: In Sec. II we present the $a b$ initio band structure of InAs and InP WZ. The multiband $k \cdot p$ model and its considerations are discussed in Sec. III. In Sec. IV, we describe our main results: (i) the fitting approach, (ii) the comparison between the $a b$ initio and $k \cdot p$ for band structure and the spin splittings, (iii) the spin expectation value for all energy bands, and (iv) the DOS extracted from the $8 \times 8$ Hamiltonian. The analytical description of the conduction band $(\mathrm{CB})$ close to the $\Gamma$ point is presented in Sec. $\mathrm{V}$, and the compact form of the valence Hamiltonian, along with its parameters, is shown in Sec. VI. Finally, in Sec. VII we present our conclusions and possible direct applications of our effective multiband model.

\section{AB INITIO BAND STRUCTURE}

The $a b$ initio electronic structure for InAs and InP in the WZ phase was calculated within the density functional theory (DFT) framework [34], using the full potential linearized augmented plane wave method implemented by the WIEN2k code [32]. To account for local and semilocal functional deficiencies to correctly describe band gaps in semiconductors, we used an efficient and accurate alternative for electronic structure calculations based on the modified Becke-Johnson (mBJ) exchange potential [35] with LDA (local density approximation) correlation [36]. It has been shown that the semilocal $\mathrm{mBJ}$ exchange potential provides prediction of band gaps of the same order [37-39] as hybrid functionals [40] and the $G W$ method [41-43]. In addition, the semilocal approach to the exchange-correlation functional is barely expensive when compared to the LDA [44] or the generalized gradient approximation [45]. The SOC is included within the second variational step [46]. Regarding the technical details of our calculations, we expanded the wave functions in atomic spheres for orbital quantum numbers up to 10; the plane wave cutoff multiplied with the smallest atomic radii equals 10 and the irreducible Brillouin zone was sampled with $600 k$ points. Further details on $a b$ initio calculations of III-V semiconductors, either with $\mathrm{ZB}$ or WZ structure, using the $\mathrm{mBJ}$ potential can be found in Ref. [47].

The particular order of cation (In) and anions (As, P) within the unit cell determines spin orientation [49]. We consider the following primitive basis vectors for corresponding hexagonal Bravais lattice, $\vec{a}_{1}=a(\sqrt{3},-1,0) / 2, \vec{a}_{2}=a(0,1,0)$, and $\vec{a}_{3}=c(0,0,1)$, where $a$ and $c$ are the WZ lattice parameters. Using the three basis vectors $\vec{a}_{i}(i=1,2,3)$ we define the following four atomic positions that form the WZ structure: $(2 / 3,1 / 3, u)$ and $(1 / 3,2 / 3,1 / 2+u)$, with $u=0$ for anion and and $u=3 / 8$ for cation. We note that in general there might be $u=3 / 8+\epsilon$ with a small dimensionless cell-internal structural parameter $\epsilon$ describing a deviation from ideal tetrahedrons as one observes for $\mathrm{SiC}$ polytypes [50]. In our calculations we considered $\epsilon=0$ since it is a rather small valued parameter [25,51]. For the lattice parameters we considered $a=4.2742 \AA$ and $c=7.025 \AA$ [52] for InAs and $a=4.1148 \AA$ and $c=6.7515 \AA$ [53] for InP.

We show the band structures obtained with WIEN2k in Fig. 1(a) for InAs and Fig. 1(b) for InP. Both compounds show a direct band gap at the $\Gamma$ point with values of $E_{g}=0.467 \mathrm{eV}$ for InAs and $E_{g}=1.494 \mathrm{eV}$ for InP. Due to the hexagonal

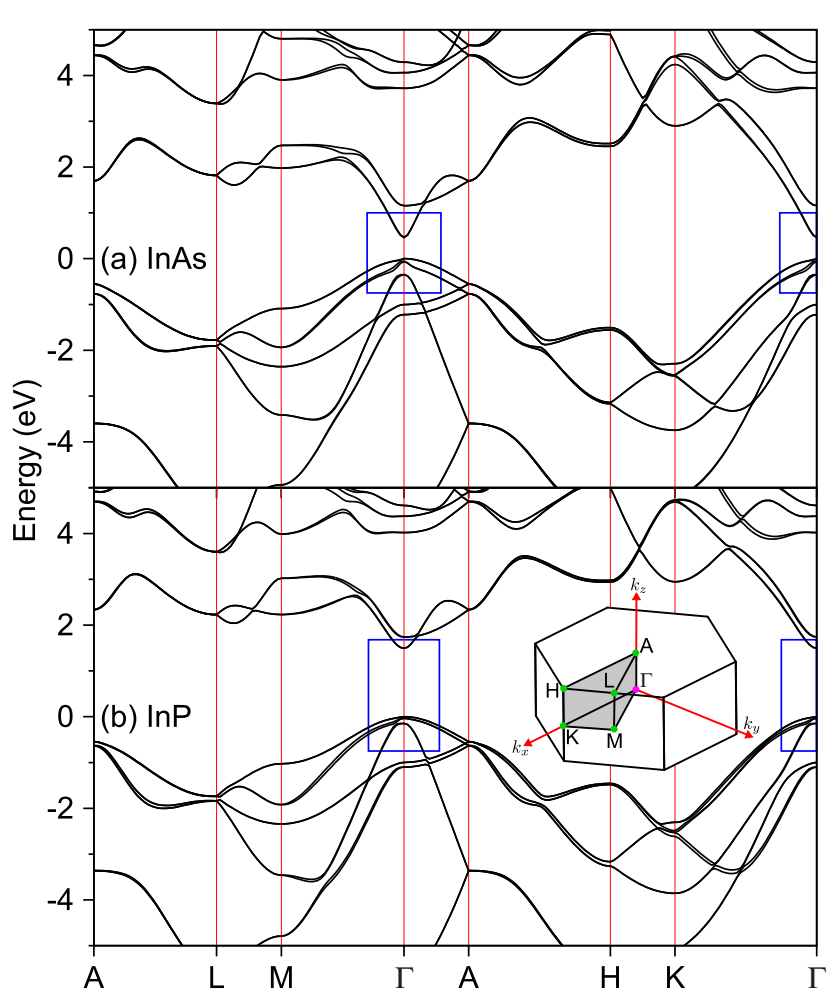

FIG. 1. Ab initio band structure along high-symmetry lines for (a) InAs and (b) InP in WZ phase. The inset shows the FBZ of WZ structure indicating the high-symmetry points. The rectangles highlight the region of interest around the $\Gamma$ point. 


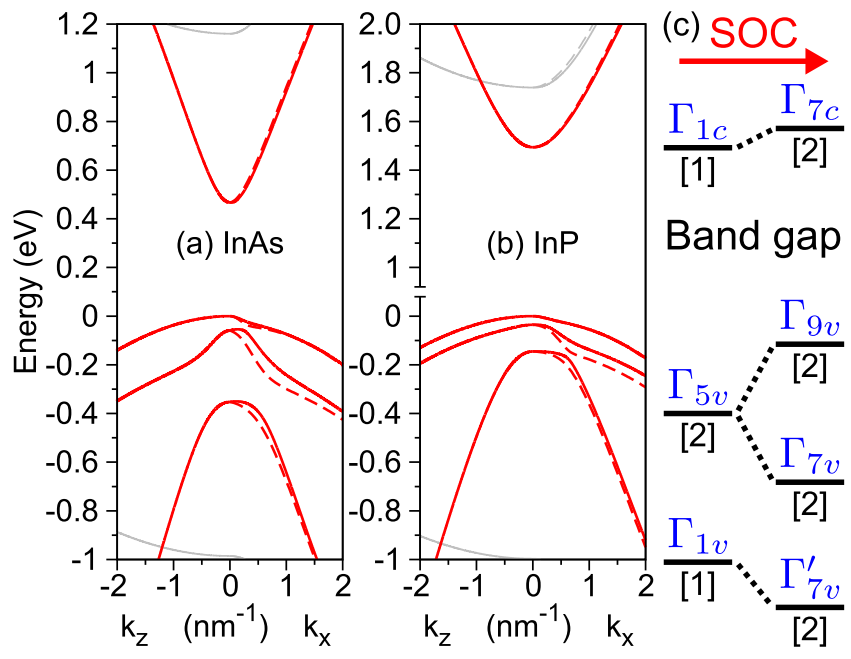

FIG. 2. Band structure for $\mathrm{WZ}$ (a) InAs and (b) InP around $\Gamma$ point for $k_{z}(\Gamma-\mathrm{A})$ and $k_{x}(\Gamma-\mathrm{K})$ directions. The solid lines indicate the outer branch and the dashed lines indicate the inner branch of the spin-split bands. The thin (gray) lines indicate the energy bands outside our range of interest. (c) Change in the irreducible representations of energy bands at $\Gamma$ point under SOC. The subscripts $v$ and $c$ added to the irreducible representations indicate valence and conduction bands, respectively, and the prime distinguishes between the two possibilities of $\Gamma_{7}$. The numbers in square brackets are the degeneracy of the bands. Our notation for the irreducible representations follows Ref. [48].

symmetry of WZ, the $\Gamma$ point, as well as the symmetry line connecting $\Gamma$-A (hexagonal axis), belongs to the $C_{6 v}$ symmetry group [54], which has only two-dimensional double-group representations. From this follows that the states along the hexagonal axis are spin degenerate [54,55]. Irreducible representations of other points in the FBZ compatible with spin are singly degenerate. Hence, except for accidental or time-reversal degeneracies at $\Gamma$ and $A$ points, spin splittings must occur for all bands.

In Figs. 2(a), for InAs, and 2(b), for InP, we display the rectangular regions of Fig. 1, i.e., a zoom of the band structure around the $\Gamma$ point. At this energy range, the anticrossing and spin-splitting features of the band structures are evident. Because of large SOC in InAs, the valence band energy levels are farther apart than InP bands and additional curvatures are present along the $k_{z}$ direction. For InP the top two valence bands along $k_{z}$ show similar curvatures and no anticrossing is visible. The effect of SOC in the energy bands at the $\Gamma$ point is shown schematically in Fig. 2(c). Without SOC, the irreducible representations belong to the simple group, while with SOC, they are referred to as the double group. This distinction is important for $k \cdot p$ perturbative approaches.

We present a comparison between our ab initio calculations and other theoretical papers in the literature in Table I. Besides the lattice constants $a$ and $c$ we compare the values of the internal parameter $u$, the energy gap $E_{g}$, and the energy difference between the top valence band $\Gamma_{9 v}$ and the other bands $\Gamma_{7 v}$ and $\Gamma_{7 v}^{\prime}$ [following the notation of Fig. 2(c)]. These energy differences are defined as $\Delta E_{97}=E\left(\Gamma_{9 v}\right)-E\left(\Gamma_{7 v}\right)$ and $\Delta E_{97}^{\prime}=E\left(\Gamma_{9 v}\right)-E\left(\Gamma_{7 v}^{\prime}\right)$. It is very common to compare the crystal field splitting energy, $\Delta_{1}$, and the SOC energy,
TABLE I. Comparison of theoretical data for InAs and InP in WZ phase. The lattice constants $a$ and $c$ are given in $\AA$, and $u$ is dimensionless. The band gap, $E_{g}$, and the valence band energy differences, $\Delta E_{97}$ and $\Delta E_{97}^{\prime}$, are given in $\mathrm{eV}$.

\begin{tabular}{cccccccc}
\hline \hline & $a$ & $c$ & $u$ & $E_{g}$ & $\Delta E_{97}$ & $\Delta E_{97}^{\prime}$ \\
\hline InAs & This study & 4.2742 & 7.0250 & 0.37500 & 0.4670 & 0.0592 & 0.3527 \\
& Ref. [23] & 4.1505 & 6.7777 & 0.37500 & 0.4810 & 0.1050 & 0.4690 \\
& Ref. [25] & 4.2570 & 6.9894 & 0.37447 & 0.4810 & 0.0573 & 0.3937 \\
& Ref. [27] & 4.2564 & 7.0046 & 0.37400 & 0.4610 & 0.0700 & 0.3640 \\
& Ref. [47] & 4.2742 & 7.0250 & 0.37422 & 0.4610 & 0.0660 & 0.3600 \\
InP & This study & 4.1148 & 6.7515 & 0.37500 & 1.4940 & 0.0354 & 0.1450 \\
& Ref. [23] & 4.2839 & 6.9955 & 0.37500 & 1.4740 & 0.0630 & 0.3480 \\
& Ref. [24] & 4.1500 & 6.9120 & 0.37100 & 1.4936 & 0.0450 & 0.2430 \\
& Ref. [25] & 4.1148 & 6.7515 & 0.37458 & 1.5760 & 0.0321 & 0.1339 \\
\hline \hline
\end{tabular}

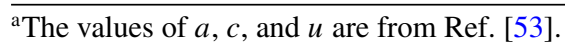

$\Delta_{S O}$; however, these parameters are usually obtained under the quasicubic approximation and do not provide a direct comparison with experiments such as $\Delta E_{97}$ and $\Delta E_{97^{\prime}}$. We can see that all the values obtained by our calculations are within the range of reported data in previous papers. We also compare experimental measurements of the energy gap with our calculated values, shown in Table II. We focused on experimental data obtained by photoluminescence measurements at low temperature of large-diameter nanowires, so that lateral quantum confinement is negligible. For both InAs (despite the reduced set of available data) and InP compounds, our calculated values of the energy gaps are consistent with the experiments. Furthermore, photoluminescence excitation measurements can probe the $\Gamma_{7 v}$ and $\Gamma_{7 v}^{\prime}$ valence bands and allow us to check our calculated values for $\Delta E_{97}$ and $\Delta E_{97}^{\prime}$ energies. To the best of our knowledge, such experiments are only available for InP. Typical values found for $\Delta E_{97}$ and $\Delta E_{97}^{\prime}$ in InP WZ are $0.044 \mathrm{eV}$ and $0.187 \mathrm{eV}$ in Ref. [9], $0.043 \mathrm{eV}$ and $0.179 \mathrm{eV}$ in Ref. [56], and $0.044 \mathrm{eV}$ and $0.182 \mathrm{eV}$ in Ref. [57]. Our calculated values for InP of $\Delta E_{97}=0.0354 \mathrm{eV}$ and

TABLE II. Comparison between theoretical and experimental values of the energy gap. We indicate the temperature of the photoluminescence measurements in parentheses.

\begin{tabular}{lcc}
\hline \hline & $\begin{array}{c}E_{g}(\mathrm{eV}) \\
\text { This study }\end{array}$ & $\begin{array}{c}E_{g}(\mathrm{eV}) \\
\text { Experiment }\end{array}$ \\
\hline InAs & 0.467 & $0.520(7 \mathrm{~K})^{\mathrm{a}}, 0.500(20 \mathrm{~K})^{\mathrm{b}}$ \\
& & $0.458(5 \mathrm{~K})^{\mathrm{c}}$ \\
InP & 1.494 & $1.492(10 \mathrm{~K})^{\mathrm{d}}, 1.494(10 \mathrm{~K})^{\mathrm{e}}$ \\
& & $1.490(20 \mathrm{~K})^{\mathrm{f}}, 1.491(4 \mathrm{~K})^{\mathrm{g}}$ \\
& & $1.493(4 \mathrm{~K})^{\mathrm{h}}, 1.488(6 \mathrm{~K})^{\mathrm{i}}$ \\
\hline \hline
\end{tabular}

\footnotetext{
${ }^{\mathrm{a}}$ Reference [60]

${ }^{\mathrm{b}}$ Reference [61]

${ }^{\mathrm{c}}$ Reference [62]

${ }^{\mathrm{d}}$ Reference [57]

${ }^{\mathrm{e}}$ Reference [56]

${ }^{\mathrm{f}}$ Reference [63]

geference [3]

${ }^{\mathrm{h}}$ Reference [64]

${ }^{\mathrm{i}}$ Reference [9].
} 
$\Delta E_{97}^{\prime}=0.145 \mathrm{eV}$ (from Table I) are also in good agreement with these experimental trends. For completeness, we provide in Appendix A the calculated values of effective masses around the $\Gamma$ point.

\section{III. $\boldsymbol{k} \cdot \boldsymbol{p}$ FORMULATION}

One alternative approach to $a b$ initio band structure calculations is the $k \cdot p$ method. In the $k \cdot p$ approach, the many-body interactions of electrons with nuclei and other electrons are described by an effective potential which has the same periodicity as the Bravais lattice of the crystal [58]. Such periodic property of the potential allows us to use Bloch's theorem for the total wave function. The single-particle Hamiltonian for the periodic part of the Bloch function, $u_{n, \vec{k}}(\vec{r})$, can be written as

$$
\begin{aligned}
\mathbf{H} & =\underbrace{\frac{p^{2}}{2 m_{0}}+V(\vec{r})}_{\mathbf{H}_{0}}+\underbrace{\frac{\hbar}{4 m_{0}^{2} c^{2}}[\vec{\nabla} V(\vec{r}) \times \vec{p}] \cdot \vec{\sigma}}_{\mathbf{H}_{\mathbf{S O}}} \\
& +\underbrace{\frac{\hbar^{2} k^{2}}{2 m_{0}}}_{\mathbf{H}_{\mathbf{k} 2}}+\underbrace{\frac{\hbar}{m_{0}} \vec{k} \cdot \vec{p}}_{\mathbf{H}_{\mathbf{k p}}}+\underbrace{\frac{\hbar^{2}}{4 m_{0}^{2} c^{2}}[\vec{\nabla} V(\vec{r}) \times \vec{k}] \cdot \vec{\sigma}}_{\mathbf{H}_{\mathbf{k S O}}},
\end{aligned}
$$

in which the different terms in the Hamiltonian are identified for convenience.

We can solve the above equation perturbatively expanding the functions $u_{n, \vec{k}}(\vec{r})$ around a specific reciprocal space point for which we know the solutions for the Hamiltonian. Since WZ InAs and InP have a direct band gap at the $\Gamma$ point, this is the chosen expansion point. The perturbative technique we use in this paper is Löwdin's formalism [59]. In this approach, the functions at the $\Gamma$ point, i.e., the basis set to expand $u_{n, \vec{k}}(\vec{r})$, are divided into classes $\mathrm{A}$ and $\mathrm{B}$. The energy bands we are interested in describing comprise the class A while the other energy bands belong to class B. The contribution of states in class B appear in second or higher orders of perturbation. The matrix elements we consider can arise from first- or secondorder perturbation, reading as

$$
H_{f, \alpha \alpha^{\prime}}^{(1)}=\left\langle\alpha\left|\mathbf{H}_{\mathbf{f}}\right| \alpha^{\prime}\right\rangle
$$

and

$$
H_{f g, \alpha \alpha^{\prime}}^{(2)}=\sum_{\beta}^{B} \frac{\left\langle\alpha\left|\mathbf{H}_{\mathbf{f}}\right| \beta\right\rangle\left\langle\beta\left|\mathbf{H}_{\mathbf{g}}\right| \alpha^{\prime}\right\rangle}{E_{\alpha \alpha^{\prime}}-E_{\beta}},
$$

where $\mathbf{H}_{\mathbf{f}}$ and $\mathbf{H}_{\mathbf{g}}$ can be any of the terms of Eq. (1), except $\mathbf{H}_{\mathbf{0}}$.

Since the unperturbed term, $\mathbf{H}_{\mathbf{0}}$, in Eq. (1) does not contain SOC effects explicitly, we consider the simple group description of the energy bands, the most usual approach in the literature [65]. Under such approximation, the states in class A belong to the irreducible representations shown on the left side of Fig. 2(c), a 4-dimensional Hilbert space, combined with the spin-1/2 angular momentum, a 2-dimensional Hilbert space. Therefore, the 8 -dimensional basis set for the $k \cdot p$
Hamiltonian in Dirac notation [66] is given by

$$
\begin{array}{rlrl}
\left|c_{1}\right\rangle & =-\frac{\left|\left(\Gamma_{5 v}^{x}+i \Gamma_{5 v}^{y}\right) \uparrow\right\rangle}{\sqrt{2}}, & & \left|c_{5}\right\rangle=-\frac{\left|\left(\Gamma_{5 v}^{x}+i \Gamma_{5 v}^{y}\right) \downarrow\right\rangle}{\sqrt{2}}, \\
\left|c_{2}\right\rangle=\frac{\left|\left(\Gamma_{5 v}^{x}-i \Gamma_{5 v}^{y}\right) \uparrow\right\rangle}{\sqrt{2}}, & \left|c_{6}\right\rangle=\left|\Gamma_{1 v} \downarrow\right\rangle, \\
\left|c_{3}\right\rangle=\left|\Gamma_{1 v} \uparrow\right\rangle, & \left|c_{7}\right\rangle=i\left|\Gamma_{1 c} \uparrow\right\rangle, \\
\left|c_{4}\right\rangle=\frac{\left|\left(\Gamma_{5 v}^{x}-i \Gamma_{5 v}^{y}\right) \downarrow\right\rangle}{\sqrt{2}}, & \left|c_{8}\right\rangle=i\left|\Gamma_{1 c} \downarrow\right\rangle
\end{array}
$$

with 1-6 representing the valence band states and 7-8 the conduction band states. Since $\Gamma_{5 v}$ is two dimensional, we identified its basis states by $\left|\Gamma_{5 v}^{x}\right\rangle \sim x$ and $\left|\Gamma_{5 v}^{y}\right\rangle \sim y$. The single arrows $(\uparrow, \downarrow)$ represent the projection of spin up and spin down, eigenvalues of the $\sigma_{z}$ Pauli matrix. The states in class B have simple group symmetries $\Gamma_{1}, \Gamma_{3}, \Gamma_{5}$, and $\Gamma_{6}$, which is the only necessary information to calculate second-order contributions.

To describe the interaction among the energy bands, we consider all terms of Eq. (1) in first-order perturbation and only the term $\mathbf{H}_{\mathbf{k p}}$ in second order. Therefore, the total matrix Hamiltonian in the basis set (4) comprises the following terms:

$$
H=H_{0}+H_{S O}^{(1)}+H_{k p}^{(1)}+H_{k S O}^{(1)}+H_{k p}^{(2)},
$$

with the explicit form of each matrix and the definition of the parameters given in Appendix B.

In Fig. 3 we show schematically the interactions for each term in the total Hamiltonian (5). The Fig. 3(a) represents the unperturbed Hamiltonian without SOC, where states $\left|c_{1(4)}\right\rangle$ and $\left|c_{2(5)}\right\rangle$ are degenerate for spin up (down). The only terms that couple different spin projections arise from $H_{S O}^{(1)}$ or $H_{k S O}^{(1)}$, Figs. 3(b) and 3(d), respectively. Usually $H_{k S O}^{(1)}$ is neglected in WZ Hamiltonians [28,29,31,67-70]. However, the explicit interactions for nonzero $k$ values are crucial to correctly describe the spin-splitting properties. We included $H_{k S O}^{(1)}$ following the approach of Dresselhaus for ZB [71]. Moreover, the coupling of $H_{S O}^{(1)}$ to other terms provides additional contributions to the spin splitting of energy bands. Besides spin-splitting properties, we want a good description of the band structure curvatures. Such effects can be modeled by linear and quadratic terms of the $H_{k p}^{(1)}$ and $H_{k p}^{(2)}$, Figs. 3(c) and 3(e), respectively. The only term that allows a $k$-dependent self-interaction of states is $H_{k p}^{(2)}$ which gives the effective mass contribution to our model.

Although the $k \cdot p$ method provides the functional form of the Hamiltonian, the parameters that describe different materials cannot be found by group theory arguments only. In order to calculate the matrix elements we would need the functions at the expansion point and also the periodic potential $V(\vec{r})$. Alternatively, we can directly fit the $k \cdot p$ Hamiltonian to the $a b$ initio band structure to extract the parameters [29,31,67,69,72].

\section{NUMERICAL FITTING OF THE $8 \times 8 \boldsymbol{k} \cdot p$ HAMILTONIAN}

We start our fitting approach by calculating the $k$ independent parameters of the Hamiltonian, i.e., the energy 

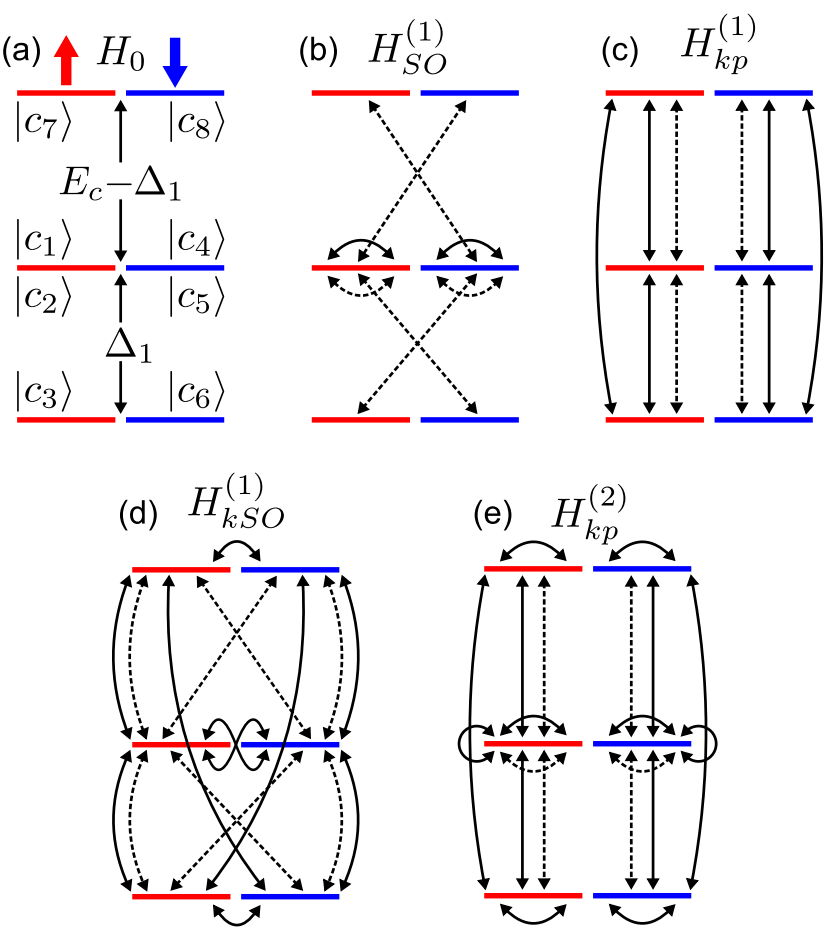

FIG. 3. Possible interactions of the Hamiltonian terms (a) $H_{0}$, (b) $H_{S O}^{(1)}$, (c) $H_{k p}^{(1)}$, (d) $H_{k S O}^{(1)}$, and (e) $H_{k p}^{(2)}$. The arrows on top of panel (a) indicate spin-up and spin-down projections of the basis states. Since $\left|c_{1(4)}\right\rangle$ and $\left|c_{2(5)}\right\rangle$ are degenerate, we indicate the interactions arising from $\left|c_{1(4)}\right\rangle$ with solid lines and the interactions arising from $\left|c_{2(5)}\right\rangle$ with dashed lines. For the other states without degeneracy we used solid lines. In panel (a) we show the energy splittings without SOC, formally defined in Appendix B.

splittings. The values for crystal field splitting, $\Delta_{1}$, and the conduction band energy, $E_{c}$, can be obtained from the ab initio calculation without SOC, which is in fact the assumption of the $k \cdot p$ perturbative theory $\left[H_{0}\right.$ term; see Fig. 3(a)]. This approach is very useful because it simplifies the calculation of the SOC energy splittings inside the valence band, $\Delta_{2}$ (coupling same spins) and $\Delta_{3}$ (coupling different spins), and the SOC between conduction and valence bands, $\Delta_{4}$. Please refer to Appendix B for the formal definition of these splitting energies. By setting the values of $\Delta_{1}$ and $E_{c}$, it possible to have $\Delta_{2} \neq \Delta_{3}$ and neglect the cubic approximation [28]. If the values of $\Delta_{1}$ and $E_{c}$ were not found without SOC, we would have to determine 5 variables having only 3 linear independent combinations of the energy bands with SOC. This approach would provide a range of possible values and further analysis would be necessary. Starting with $\Delta_{1}$ and $E_{c}$ values without SOC, we obtained four different solution sets for the SOC splitting energies since $\Delta_{3}$ and $\Delta_{4}$ are off-diagonal terms in the Hamiltonian and can assume positive or negative values with the same magnitude. At the $\Gamma$ point any of these solution sets give the same eigenvalues; therefore we set $\Delta_{3}$ to be positive [23-25,27] and investigated the effect of positive and negative values of $\Delta_{4}$.

Before starting the fitting of the $k$-dependent parameters, it is important to define the fitting region we are interested in, which is connected to the limits of our $k \cdot p$ model. Basically, in order to describe as precisely as possible the 8 bands we are interested in, we should stay in a region away from the influence of remote bands, roughly $k \sim 1.5 \mathrm{~nm}^{-1}$; see Figs. 2(a) and 2(b). We also want to have a nice description of the anticrossings in the band structure around $k \sim 0.5 \mathrm{~nm}^{-1}$. Furthermore, in the $k \cdot p$ Hamiltonian $k_{x}$ and $k_{y}$ directions are equivalent, but this is not the case for the $a b$ initio band structure. Around $k \sim 1.0 \mathrm{~nm}^{-1}$, the $a b$ initio band structures along the $\Gamma-\mathrm{K}$ and $\Gamma-\mathrm{M}$ directions are different, especially the spin splitting, which is another feature to be described. Therefore, it is reasonable to set the goal of our fitting at $k=1.0 \mathrm{~nm}^{-1}$ to find the best parameter set that describes the $a b$ initio band structure around the $\Gamma$ point for all 8 bands.

To increase the accuracy of our parameter sets, we fitted, simultaneously, the energy bands in multiple directions of the FBZ $(\Gamma-\mathrm{K}, \Gamma-\mathrm{M}, \Gamma-\mathrm{A}, \Gamma-\mathrm{H}$, and $\Gamma-\mathrm{L})$. The fitting algorithm was developed using the LMFIT [73] package of Python assuming several minimization methods available. We noticed that the minimization methods behave differently and usually provide different parameter sets. After an initial fit, we chose the best parameter set and used it as input for a new fit using all minimization methods again. To find the best fit, the band structures and spin splittings are compared by their residue [74] up to $k=1.0 \mathrm{~nm}^{-1}$ for all directions. The best parameter sets for InAs and InP found by our fitting approach are presented in Table III.

TABLE III. Parameter sets of the $8 \times 8$ Hamiltonian for InAs and InP WZ. The energy splittings are given in $\mathrm{eV}$, linear parameters in $\mathrm{eV} \AA$, and second-order parameters in units of $\hbar^{2} / 2 m_{0}$.

\begin{tabular}{lrr}
\hline \hline Parameter & InAs & \multicolumn{1}{c}{ InP } \\
\hline Energy splittings & & \\
$\Delta_{1}$ & 0.1003 & 0.0945 \\
$\Delta_{2}$ & 0.1023 & 0.0279 \\
$\Delta_{3}$ & 0.1041 & 0.0314 \\
$\Delta_{4}$ & 0.0388 & 0.0411 \\
$E_{c}$ & 0.6649 & 1.6142 \\
Linear parameters & & \\
$A_{7}$ & -0.4904 & -0.1539 \\
$P_{1}$ & 8.3860 & 7.6349 \\
$P_{2}$ & 6.8987 & 5.5651 \\
$\alpha_{1}$ & -0.0189 & 0.2466 \\
$\alpha_{2}$ & -0.2892 & -0.2223 \\
$\alpha_{3}$ & -0.5117 & -0.2394 \\
$\beta_{1}$ & -0.0695 & -0.0481 \\
$\beta_{2}$ & -0.2171 & -0.1386 \\
$\gamma_{1}$ & 0.5306 & 0.2485 \\
Second order parameters & & \\
$A_{1}$ & 1.5726 & -1.0419 \\
$A_{2}$ & -1.6521 & -0.9645 \\
$A_{3}$ & -2.6301 & -0.0694 \\
$A_{4}$ & 0.5126 & -1.2760 \\
$A_{5}$ & 0.1172 & -1.1024 \\
$A_{6}$ & 1.3103 & -0.5677 \\
$e_{1}$ & -3.2005 & -0.5732 \\
$e_{2}$ & 0.6363 & 2.4084 \\
$B_{1}$ & -2.3925 & -7.7892 \\
$B_{2}$ & 2.3155 & 4.3981 \\
$B_{3}$ & -1.7231 & 9.1120 \\
\hline \hline & & \\
\hline & & \\
\hline
\end{tabular}



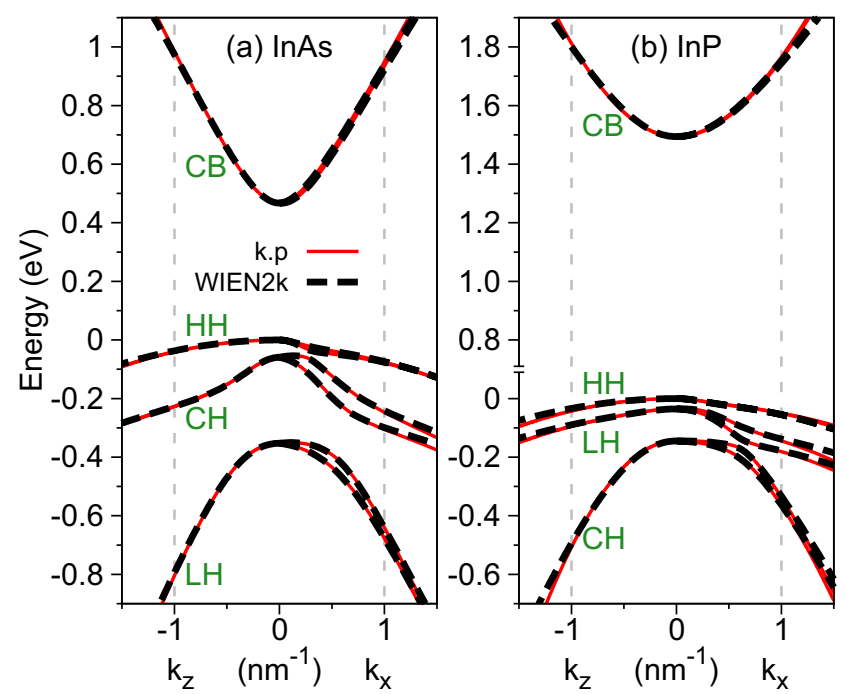

FIG. 4. Comparison of band structures calculated from the fitted $k \cdot p$ model (solid lines) and the $a b$ initio WIEN2k (dashed lines) for (a) InAs and (b) InP. The vertical dashed lines at $1.0 \mathrm{~nm}^{-1}$ indicate the borders of the fitting range.

In Fig. 4, we present the comparison between the fitted and WIEN2k ab initio band structures along $k_{z}$ and $k_{x}$ for InAs and InP. All the important features around the $\Gamma$ point, i.e., anticrossings and spin splittings, are captured by our model. We notice a good agreement up to $k=1.0 \mathrm{~nm}^{-1}$ with small deviations above it, indicating that we are reaching the region where the influence of remote energy bands becomes important. We labeled the valence bands according to the composition of states at the $\Gamma$ point. Following Chuang and Chang's notation [75], HH is purely composed of $\left|c_{1(4)}\right\rangle$ states, LH has more contribution from $\left|c_{2(5)}\right\rangle$ than $\left|c_{3(6)}\right\rangle$ states, and $\mathrm{CH}$ has more contribution from $\left|c_{3(6)}\right\rangle$ than $\left|c_{2(5)}\right\rangle$ states. Since this analysis is usually performed without the $\Delta_{4}$ parameter, we also calculated $\Delta_{2}$ and $\Delta_{3}$ considering $\Delta_{4}=0$ and we found that the same labeling holds (these values are shown in Sec. VI). Furthermore, we also compared the $k \cdot p$ composition with the projection to atomic orbitals of the ab initio wave functions and the same trends can be noticed. The labeling order of $\mathrm{CH}-\mathrm{LH}$ in InAs is due to the values of SOC splitting energies, which are slightly larger than the crystal field splitting. For InP, the crystal field splitting is dominant leading to $\mathrm{LH}-\mathrm{CH}$ ordering. Although this labeling of the valence band can be confusing, it is very useful to extract optical trends from the band-egde transitions. For instance, if we take into account optical transitions arising from the top two valence bands, we can expect InP light polarization to be more in-plane due to $\mathrm{LH}$ contribution than InAs due to $\mathrm{CH}$ contribution. Finally, for the conduction band of both InAs and InP we simply label it $\mathrm{CB}$, short notation for conduction band; $\mathrm{CB}$ is mainly composed of $\left|c_{7(8)}\right\rangle$ states.

Let us take a closer look at the spin-splitting properties obtained from the $k \cdot p$ model and the ab initio. We show the comparison between the two methods in Fig. 5 for InAs and InP along the $k_{x}$ direction. Similarly to the band structure, we have a good agreement up to $k=1.0 \mathrm{~nm}^{-1}$ with deviations above this region. The intricate behaviors, i.e., the appearance of maxima and crossings between $\mathrm{HH}$ spin-split bands, are also
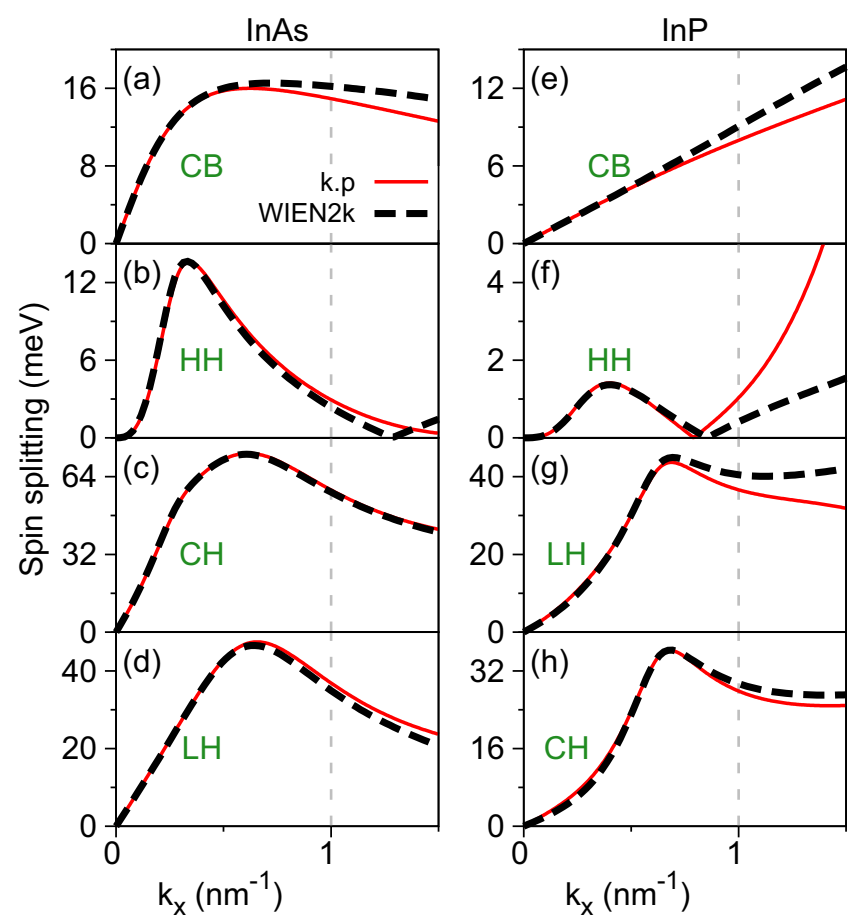

FIG. 5. Comparison of the spin splittings along $k_{x}$ for the energy bands (a) $\mathrm{CB}$, (b) $\mathrm{HH}$, (c) $\mathrm{CH}$, and (d) $\mathrm{LH}$ of InAs and (e) $\mathrm{CB}$, (f) $\mathrm{HH},(\mathrm{g}) \mathrm{LH}$, and (h) $\mathrm{CH}$ of InP. The line schemes follow Fig. 4.

described by our model. All these spin-splitting characteristics have only one physical origin, the BIA of the WZ structure. The strength of SOC is greater in InAs than InP, visible at the peak values and positions. From the largest to the smallest values of the spin splitting, we have $\mathrm{CH}(\mathrm{LH}), \mathrm{LH}(\mathrm{CH}), \mathrm{CB}$, and $\mathrm{HH}$ for InAs (InP). Furthermore, a linear behavior is maintained for InP CB throughout the fitting region. For InAs, this linear behavior is attained only in a small region close to the $\Gamma$ point. In Appendix $\mathrm{C}$, we present the band structure and spin splittings for the other FBZ directions used in the fitting.

Another feature we investigated is the spin orientation, i.e., the spin expectation value, $\langle\vec{\sigma}\rangle$, for the different energy bands, presented in Fig. 6 for the $k_{x} k_{y}$ plane $\left(k_{z}=0\right)$. We chose the constant-energy contours to be $E_{n}\left(k_{x}=0.5 \mathrm{~nm}^{-1}, k_{y}=\right.$ $0, k_{z}=0$ ) of the outer branch, i.e., $E_{\mathrm{CB}} \sim 630.0 \mathrm{meV}, E_{\mathrm{HH}} \sim$ $-37.2 \mathrm{meV}, E_{\mathrm{CH}} \sim-123.0 \mathrm{meV}, E_{\mathrm{LH}} \sim-391.8 \mathrm{meV}$ for InAs and $E_{\mathrm{CB}} \sim 1563.5 \mathrm{meV}, E_{\mathrm{HH}} \sim-21.9 \mathrm{meV}, E_{\mathrm{LH}} \sim$ $-75.0 \mathrm{meV}, E_{\mathrm{CH}} \sim-156.7 \mathrm{meV}$ for InP. We found that all the investigated energy bands show a Rashba-like spin texture. For InAs, the bands $\mathrm{CB}, \mathrm{HH}$, and $\mathrm{CH}$ have the same spin texture, i.e., $\mathrm{CW}(\mathrm{CCW})$ orientation for the inner (outer) branch, while LH has the CCW (CW) orientation for the inner (outer) branch. In other words, the top two valence bands have the same spin texture while the third valence band has the opposite. For InP, the same spin texture holds, even though the labeling of $\mathrm{CH}$ and LH is reversed. The spin textures calculated with the $k \cdot p$ model were also checked with the $a b$ initio calculations.

Performing the fitting approach with the negative sign of $\Delta_{4}$ we obtained the same behavior of the band structure and the spin splittings, but with a reversed orientation in the spin texture, i.e., $\mathrm{CW}$ orientation becomes $\mathrm{CCW}$ and vice versa for 

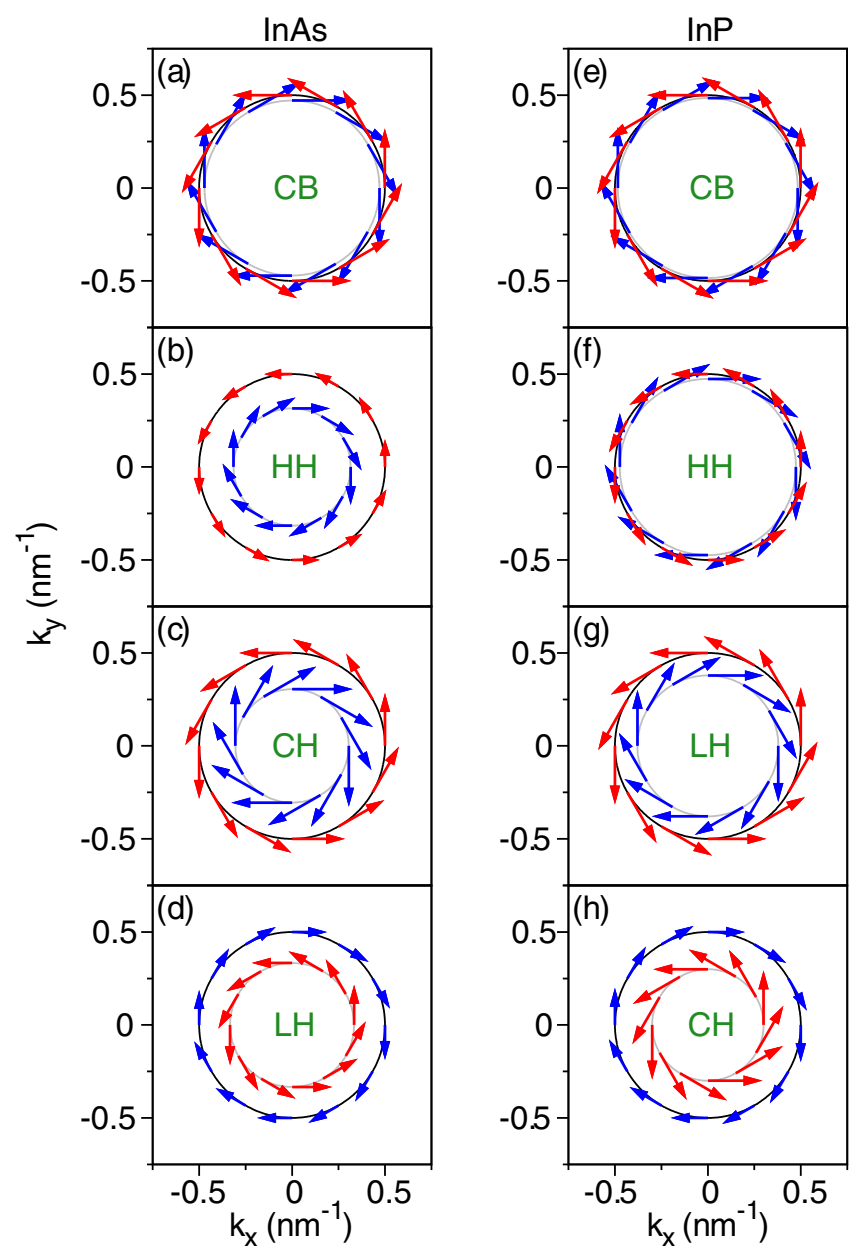

FIG. 6. Spin texture in the $k_{x} k_{y}$ plane $\left(k_{z}=0\right)$ for the energy bands (a) $\mathrm{CB}$, (b) $\mathrm{HH}$, (c) $\mathrm{CH}$, and (d) $\mathrm{LH}$ of InAs and (e) $\mathrm{CB}$, (f) $\mathrm{HH},(\mathrm{g}) \mathrm{LH}$, and (h) $\mathrm{CH}$ of InP. The blue arrows indicate clockwise orientation while the red arrows indicate counterclockwise orientation. The amplitudes of all arrows are multiplied by 0.3 to fit the figure. The constant-energy contours are also drawn in the figure in black for the outer branches and in gray for the inner branches.

all bands. Specifically, we found that starting with negative value of $\Delta_{4}$, the signs of parameters $A_{7}, \alpha_{1}, \alpha_{2}, \alpha_{3}, \gamma_{1}, B_{1}, B_{2}$, and $B_{3}$ are changed, but not their amplitude. This change in the spin texture is a feature expected from $a b$ initio regarding the cation and anion positions within the crystal unit cell [49] and it is reflected in our $k \cdot p$ model and parameters. Therefore, in order to provide reliable parameter sets for $k \cdot p$ Hamiltonians, not only the band structure and the spin splittings should be checked but also the spin orientation. We would like to emphasize that all these features were systematically checked in this study.

\section{A. Density of states and carrier density}

Relying on the effective $8 \times 8 k \cdot p$ Hamiltonian, it is straightforward to calculate a smooth DOS using a fine 3dimensional $(3 \mathrm{D})$ mesh of $k$ points $(300 \times 300 \times 300)$ without much computational effort. In Fig. 7(a) we show the DOS for the conduction band of InAs and InP. For comparison, we also
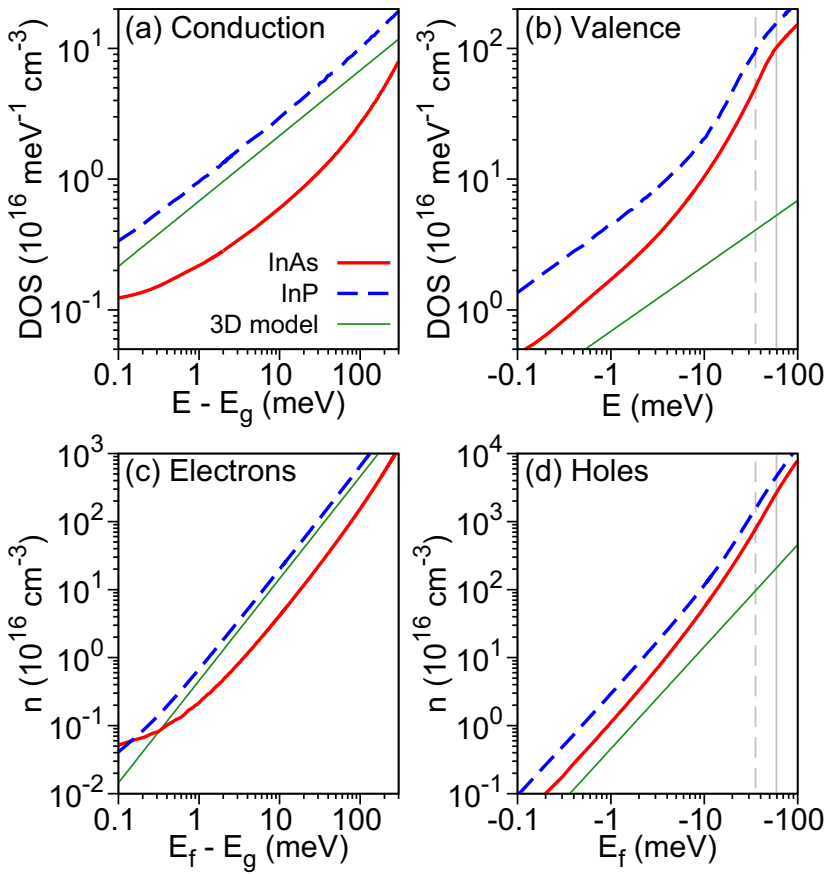

FIG. 7. Calculated DOS for (a) conduction band and (b) valence band of InAs, InP, and the 3D parabolic band model using an effective mass of $m^{*}=0.1$. Carrier density, $n$, as a function of Fermi energy, $E_{f}$, for (c) electrons and (d) holes obtained by the integration of the DOS in panels (a) and (b), respectively. The dashed vertical lines in panels (b) and (d) indicate the LH energy at $\Gamma$ point for InP while the solid vertical lines indicate the $\mathrm{CH}$ energy energy at $\Gamma$ point for InAs.

show the DOS for the 3D parabolic band model [DOS $(E) \propto$ $\sqrt{E}]$, which is just a straight line in the $\log -\log$ scale. Due to the complex behavior of the InAs and InP conduction bands, we clearly see deviations from the linear behavior, especially for InAs. For the DOS of the valence band, presented in Fig. 7(b), the deviations from the parabolic model are much more visible, showing explicitly the need of a multiband approach. When the valence band energy approaches the $\mathrm{CH}(\mathrm{LH})$ region of InAs (InP), the DOS changes its curvature. Moreover, the valence band DOS is approximately one order of magnitude larger than the DOS of the conduction band, a behavior attributed to the small curvatures of the valence bands, i.e., large effective masses for holes (in a single band picture). Integrating the DOS we obtain the carrier density as a function of the Fermi energy, presented in Figs. 7(c) and 7(d) for electrons and holes, respectively. Typically, InP supports larger values of the carrier density than InAs. For instance, for $100 \mathrm{meV}$ above the energy gap $\sim 1.6 \times 10^{18} \mathrm{~cm}^{-3}$ for InAs and $\sim 6.5 \times 10^{18} \mathrm{~cm}^{-3}$. In the Supplemental Material [76] we provide a curve fitting of the carrier density curves that can be directly applied to predict the carrier concentration or the Fermi energy without the explicit DOS calculation using the $8 \times 8 k \cdot p$ Hamiltonian.

\section{ANALYTICAL DESCRIPTION FOR CONDUCTION BAND}

Since the conduction band has a predominant contribution of $\left|c_{7(8)}\right\rangle$ states, it is useful to provide an analytical description that holds for small regions close to the $\Gamma$ point that can 
be easily used in spin dynamics studies. We apply Löwdin's approach again, but now dividing the basis states $\mathrm{A}$ [Eq. (4)] of the full matrix into two new classes $\mathrm{A}^{\prime}\left(\left|c_{7,8}\right\rangle\right)$ and $\mathrm{B}^{\prime}\left(\left|c_{1, \cdots, 6}\right\rangle\right.$. Using only the terms we already calculated in the full Hamiltonian as contribution to the effective Hamiltonian, this Löwdin approach is usually referred to as folding down $[59,68]$. The effective Hamiltonian for the first-order folding down, keeping terms up to $k^{3}$, can be written as

$$
H_{\mathrm{CB}}=M(\vec{k}) \mathcal{I}_{2}+\vec{\Omega}(\vec{k}) \cdot \vec{\sigma},
$$

in which $\mathcal{I}_{2}$ is a $2 \times 2$ identity matrix and $M$ is the effective mass term given by

$$
M=E_{g}+m_{z} k_{z}^{2}+m_{x y}\left(k_{x}^{2}+k_{y}^{2}\right),
$$

with the coefficients $m_{z}$ and $m_{x y}$ given by

$$
\begin{aligned}
m_{z}= & e_{1}+\frac{P_{1}^{2}}{E_{c}}+\frac{2 \beta_{1}^{2}}{E_{c}-\Delta_{1}+\Delta_{2}}+\frac{2 \Delta_{4}^{2}\left(A_{1}+A_{3}\right)}{\left(E_{c}-\Delta_{1}+\Delta_{2}\right)^{2}} \\
m_{x y}= & e_{2}+\frac{1}{2} \frac{\left(P_{2}+\beta_{1}\right)^{2}}{E_{c}-\Delta_{1}+\Delta_{2}}+\frac{1}{2} \frac{\left(P_{2}-\beta_{1}\right)^{2}}{E_{c}-\Delta_{1}-\Delta_{2}} \\
& +\frac{\beta_{2}^{2}}{E_{c}}+\frac{2 \Delta_{4}^{2}\left(A_{2}+A_{4}\right)}{\left(E_{c}-\Delta_{1}+\Delta_{2}\right)^{2}}
\end{aligned}
$$

The SOC field $\vec{\Omega}(\vec{k})$ is written as

$$
\vec{\Omega}(\vec{k})=\left[\alpha+\gamma_{z} k_{z}^{2}+\gamma_{x y}\left(k_{x}^{2}+k_{y}^{2}\right)\right]\left[\begin{array}{c}
k_{y} \\
-k_{x} \\
0
\end{array}\right],
$$

with linear and cubic coefficients given by

$$
\begin{aligned}
\alpha & =-\gamma_{1}+\frac{2 \Delta_{4}\left(P_{2}+\beta_{1}\right)}{E_{c}-\Delta_{1}+\Delta_{2}}, \\
\gamma_{z} & =2 \sqrt{2} \beta_{1} B_{3}-\frac{2 \beta_{2} B_{1}}{E_{c}}+\frac{2 \Delta_{4}\left(P_{2}+\beta_{1}\right)\left(A_{1}+A_{3}\right)}{\left(E_{c}-\Delta_{1}+\Delta_{2}\right)^{2}}, \\
\gamma_{x y} & =-\frac{2 \beta_{2} B_{2}}{E_{c}}+\frac{2 \Delta_{4}\left(P_{2}+\beta_{1}\right)\left(A_{2}+A_{4}\right)}{\left(E_{c}-\Delta_{1}+\Delta_{2}\right)^{2}} .
\end{aligned}
$$

This analytical approach for the conduction band provides a reasonable description up to $0.2 \mathrm{~nm}^{-1}$ for InAs and $0.6 \mathrm{~nm}^{-1}$ for InP, which is roughly $100 \mathrm{meV}$ above the energy gap in both cases. The numerical values of $m_{z}, m_{x y}, \alpha, \gamma_{z}$, and $\gamma_{x y}$ can be obtained by replacing the parameters with values presented in Table III. Setting the $k$-dependent SOC parameters $\gamma_{1}$ and $\beta_{1}$ to zero, we recover the analytical linear splitting found in Ref. [68]. Our approach has the advantage of also providing the analytical description of the cubic terms. For additional corrections to the cubic term, it is possible to include higher order terms in the folding-down approach. The comparison to $a b$ initio data using the analytical expressions presented in this section can be found in the Supplemental Material [76].

\section{COMPACT DESCRIPTION FOR VALENCE BAND}

Because of the coupling from the crystal field and SOC energies, the best simplified description for the valence band is simply neglecting the coupling with the conduction band, thus leading to a $6 \times 6$ matrix. It is possible to write this $6 \times 6$ Hamiltonian in a compact form using direct products of $3 \times 3$ (orbital) and $2 \times 2$ (spin) matrices [28,72]. In the basis set $\left\{\left|c_{1}\right\rangle,\left|c_{3}\right\rangle,\left|c_{2}\right\rangle,\left|c_{5}\right\rangle,\left|c_{6}\right\rangle,\left|c_{4}\right\rangle\right\}$, the compact form of valence band is written as

$$
\begin{aligned}
H_{\mathrm{VB}}= & \Delta_{1} J_{z}^{2} \mathcal{I}_{2}+\Delta_{2} J_{z} \sigma_{z}+\sqrt{2} \Delta_{3}\left(J_{+} \sigma_{-}+J_{-} \sigma_{+}\right) \\
& +\left(A_{1} \mathcal{I}_{3}+A_{3} J_{z}^{2}\right) k_{z}^{2} \mathcal{I}_{2}+\left(A_{2} \mathcal{I}_{3}+A_{4} J_{z}^{2}\right)\left(k_{x}^{2}+k_{y}^{2}\right) \mathcal{I}_{2} \\
& -A_{5}\left(J_{+}^{2} k_{-}^{2}+J_{-}^{2} k_{+}^{2}\right) \mathcal{I}_{2} \\
& -2 A_{6} k_{z}\left(\left\{J_{z} J_{+}\right\} k_{-}+\left\{J_{z}, J_{-}\right\} k_{+}\right) \mathcal{I}_{2} \\
& +i A_{7}\left(J_{+} k_{-}-J_{-} k_{+}\right) \mathcal{I}_{2} \\
& +i \sqrt{2} \alpha_{1}\left[\left\{J_{z} J_{-}\right\}\left(\sigma_{z} k_{+}-2 \sigma_{+} k_{z}\right)\right. \\
& \left.-\left\{J_{z} J_{+}\right\}\left(\sigma_{z} k_{-}-2 \sigma_{-} k_{z}\right)\right] \\
& +i\left[\left(\alpha_{3}-\alpha_{2}\right) J_{z}^{2}-\alpha_{3} \mathcal{I}_{3}\right]\left(\sigma_{+} k_{-}-\sigma_{-} k_{+}\right)
\end{aligned}
$$

with $\quad\left\{J_{a} J_{b}\right\}=\frac{1}{2}\left(J_{a} J_{b}+J_{b} J_{a}\right), \quad J_{ \pm}=\frac{1}{\sqrt{2}}\left(J_{x} \pm J_{y}\right), \quad \sigma_{ \pm}=$ $\frac{1}{2}\left(\sigma_{x} \pm \sigma_{y}\right)$, and $k_{ \pm}=k_{x} \pm i k_{y}$. The definitions of $J_{x}, J_{y}$, and $J_{z}$ matrices can be found in Appendix A [Eq. (A3)] of Ref. [28]. The matrix $\mathcal{I}_{n}$ is an $n$-dimensional identity. The product of $3 \times 3$ matrices $(A)$ with $2 \times 2$ matrices $(a)$ is defined here as

$$
A a=\left[\begin{array}{ll}
a_{11} A & a_{12} A \\
a_{22} A & a_{22} A
\end{array}\right] .
$$

To obtain the best parameter sets that describe the ab initio band structure, we performed the same fitting approach as described in Sec. IV. We found that, in order to attain the monotonic behavior of the bands, some features of the band structure or the spin splittings are not matched as precisely as the results using the $8 \times 8$ Hamiltonian. For instance, the band structures and the spin splittings for InP look reasonable; however, the spin orientation for $\mathrm{LH}$ and $\mathrm{CH}$ shows opposite trends. For InAs, the spin texture follows the correct behavior; however, the band structure and the spin splittings show the $a b$ initio features shifted to higher $k$ values. We show the fitting results for the $6 \times 6$ description and the comparison to $a b$ initio in the Supplemental Material [76]. The best parameter sets are displayed in Table IV. We would like to emphasize that the

TABLE IV. Parameter sets of the $6 \times 6$ valence band Hamiltonian for InAs and InP WZ. The units follow Table III.

\begin{tabular}{lrr}
\hline \hline Parameter & InAs & \multicolumn{1}{c}{ InP } \\
\hline Energy splittings & & \\
$\Delta_{1}$ & 0.1003 & 0.0945 \\
$\Delta_{2}$ & 0.1038 & 0.0286 \\
$\Delta_{3}$ & 0.1037 & 0.0310 \\
Linear parameters & & \\
$A_{7}$ & -0.5565 & -0.0917 \\
$\alpha_{1}$ & -0.0237 & 0.3309 \\
$\alpha_{2}$ & -0.0758 & -0.0702 \\
$\alpha_{3}$ & -0.0967 & -0.0521 \\
Second order parameters & & \\
$A_{1}$ & -17.2689 & -10.5414 \\
$A_{2}$ & -1.2047 & -1.4542 \\
$A_{3}$ & 16.6637 & 9.4589 \\
$A_{4}$ & -7.6202 & -3.2741 \\
$A_{5}$ & -5.9281 & 3.9468 \\
$A_{6}$ & -7.3872 & -0.2759 \\
\hline \hline
\end{tabular}


most reliable approach is to use the $8 \times 8$ Hamiltonian with parameter sets we provide in Sec. IV.

\section{CONCLUSIONS}

In this paper, we have calculated the band structure of InAs and InP in the WZ phase using the WIEN2k $a b$ initio code. Both compounds have a direct band gap at the $\Gamma$ point with the SOC effects clearly larger for InAs than InP. Our calculations are consistent with theoretical and experimental reported values in the literature. In order to describe the band structure around the FBZ center, we developed a multiband $8 \times 8 k \cdot p$ model for the first conduction band and the top three valence bands, including spin. The fitted parameters we obtained for the $k \cdot p$ Hamiltonian recover the important features of the $a b$ initio band structure with good agreement up to $1.0 \mathrm{~nm}^{-1}$ for multiple directions in the FBZ. Due to the stronger SOC of InAs compared to its crystal field splitting, the labeling of $\mathrm{LH}$ and $\mathrm{CH}$ energy bands at the $\Gamma$ point is reversed from InP. Regarding the spin-splitting properties, we included the $k$-dependent SOC term in the Hamiltonian, which is usually neglected in the literature. This term, combined with the other indirect couplings in the Hamiltonian, allowed the description of the spin-splitting properties farther away from the vicinity of the $\Gamma$ point. Our model captured all the important features including the description of maxima values and also the crossing between the spin-split bands (clearly seen in the $\mathrm{HH}$ band of InP, for instance). All these intricate behaviors of spin splitting have a unique physical origin, the BIA of the WZ structure. Furthermore, we calculated the in-plane spin orientation, i.e., the spin expectation value, of the energy bands and found that they all have a Rashba-like spin texture, either CW or CCW. This spin orientation was also compared to $a b$ initio data to correctly identify the signs of the parameters in the Hamiltonian. Using our multiband $k \cdot p$ Hamiltonian, we obtained the DOS for conduction and valence bands and calculated the carrier density as a function of the Fermi energy. In addition to the $8 \times 8$ Hamiltonian, we present analytical expressions for the effective masses and the SOC field of conduction band which holds in the vicinities of the $\Gamma$ point. For completeness, we also fitted the $6 \times 6 k \cdot p$ model for the valence band to the $a b$ initio data. We emphasize that the best effective description that matches our $a b$ initio calculations is the full $8 \times 8 k \cdot p$ Hamiltonian.

In conclusion, we provided in this study robust $k \cdot p$ models and parameter sets that can be straightforwardly applied to investigate novel effects in InAs- and InP-based nanostructures. For instance, polytypic systems of mixed WZ and $\mathrm{ZB}$ have already been demonstrated experimentally for both InAs and InP with great growth control of the different phases [2] and there are also theoretical models to treat such systems $[10,30,77]$. Furthermore, InAs nanowires are also a platform for studies in Majorana fermions [15]. One of the key ingredients for such realization is the presence of a robust SOC to split the energy bands, a feature already included in our model. Finally, it is straightforward to include strain effects by using the well-established WZ strain Hamiltonian [28,30] combined with the elastic constants and deformation potentials for InAs and InP in the WZ phase already reported in the literature [26,78-80].

\section{ACKNOWLEDGMENTS}

The authors acknowledge financial support from CAPES PVE (Grant No. 88881.068174/2014-01), CNPq (Grants No. 149904/2013-4, No. 88887.110814/2015-00, and No. 304289/2015-9), DFG SFB 689, and FAPESP (Grant No. 2012/05618-0). P.E.F.J. thanks A. Polimeni for suggesting the calculation of effective masses.

\section{APPENDIX A: EFFECTIVE MASSES}

Very close to the $\Gamma$ point we can estimate the effective masses by fitting a parabolic dispersion to the ab initio data. In Table $\mathrm{V}$, we show the values of effective masses along the $k_{z}$ and $k_{x}$ directions for the highlighted energy bands of Figs. 2(a) and 2(b). For the $k_{x}$ direction, we calculated the effective masses assuming the average value of the spin-splitting bands, i.e., $\left(E_{o}+E_{i}\right) / 2$ with the subindex $o(i)$ indicating the outer (inner) branch.

\section{APPENDIX B: HAMILTONIAN TERMS AND PARAMETERS}

In this Appendix, we present the matrix forms of all terms in Eq. (5) and the definition of parameters using the simple group formalism.

Matrix representation of $H_{0}$ :

$$
H_{0}=\operatorname{diag}\left[\Delta_{1}, \Delta_{1}, 0, \Delta_{1}, \Delta_{1}, 0, E_{c}, E_{c}\right],
$$

with the definitions $\left\langle\Gamma_{5 v}^{x}\left|H_{0}\right| \Gamma_{5 v}^{x}\right\rangle=\left\langle\Gamma_{5 v}^{y}\left|H_{0}\right| \Gamma_{5 v}^{y}\right\rangle=\Delta_{1}$, $\left\langle\Gamma_{1 v}\left|H_{0}\right| \Gamma_{1 v}\right\rangle=0$, and $\left\langle\Gamma_{1 c}\left|H_{0}\right| \Gamma_{1 c}\right\rangle=E_{c}$.

The zero energy is defined without SOC for states $\left|c_{3}\right\rangle$ and $\left|c_{6}\right\rangle$. The parameter $\Delta_{1}$ is the crystal field splitting energy, which arises due to the $\mathrm{WZ}$ anisotropy between the $x y$ plane and $z$ direction, and the conduction band energy is denoted by the parameter $E_{c}$. It is possible to make the connection with the energy gap including SOC coupling by writing $E_{c}=E_{g}+\Delta_{c}$, for instance. It is also convenient to consider a diagonal energy offset to set the top valence band at zero energy.

TABLE V. Effective masses for InAs and InP along $k_{z}\left(m_{\|}^{*}\right)$ and $k_{x}\left(m_{\perp}^{*}\right)$ for the highlighted bands of Figs. 2(a) and 2(b). The effective masses were obtained by fitting a parabola up to $2 \%$ of the FBZ along the specified directions.

\begin{tabular}{lrrrrr}
\hline \hline & \multicolumn{2}{c}{ InAs } & & \multicolumn{2}{c}{$\operatorname{InP}$} \\
\cline { 2 - 3 } \cline { 5 - 6 } & \multicolumn{1}{c}{$m_{\|}^{*}$} & $m_{\perp}^{*}$ & & \multicolumn{1}{c}{$m_{\|}^{*}$} & \multicolumn{1}{c}{$m_{\perp}^{*}$} \\
\hline$\Gamma_{7 c}$ & 0.0370 & 0.0416 & & 0.0947 & 0.1183 \\
$\Gamma_{9 v}$ & -0.9738 & -0.0795 & & -1.0646 & -0.2091 \\
$\Gamma_{7 v}$ & -0.0551 & -0.1046 & & -0.3064 & -0.1988 \\
$\Gamma_{7 v}^{\prime}$ & -0.0863 & -0.1838 & & -0.1016 & -0.4887 \\
\hline \hline
\end{tabular}


Matrix representation of $H_{S O}^{(1)}$ :

$$
H_{S O}^{(1)}=\left[\begin{array}{cccccccc}
\Delta_{2} & 0 & 0 & 0 & 0 & 0 & 0 & 0 \\
0 & -\Delta_{2} & 0 & 0 & 0 & \sqrt{2} \Delta_{3} & 0 & i \sqrt{2} \Delta_{4} \\
0 & 0 & 0 & 0 & \sqrt{2} \Delta_{3} & 0 & 0 & 0 \\
0 & 0 & 0 & \Delta_{2} & 0 & 0 & 0 & 0 \\
0 & 0 & \sqrt{2} \Delta_{3} & 0 & -\Delta_{2} & 0 & i \sqrt{2} \Delta_{4} & 0 \\
0 & \sqrt{2} \Delta_{3} & 0 & 0 & 0 & 0 & 0 & 0 \\
0 & 0 & 0 & 0 & -i \sqrt{2} \Delta_{4} & 0 & 0 & 0 \\
0 & -i \sqrt{2} \Delta_{4} & 0 & 0 & 0 & 0 & 0 & 0
\end{array}\right],
$$

with the definitions

$$
\begin{aligned}
& \Delta_{2}=\frac{i \hbar}{4 m_{0}^{2} c^{2}}\left\langle\Gamma_{5 v}^{x}\left|\frac{\partial V}{\partial x} p_{y}-\frac{\partial V}{\partial y} p_{x}\right| \Gamma_{5 v}^{y}\right\rangle, \\
& \Delta_{3}=\frac{i \hbar}{4 m_{0}^{2} c^{2}}\left\langle\Gamma_{5 v}^{y}\left|\frac{\partial V}{\partial y} p_{z}-\frac{\partial V}{\partial z} p_{y}\right| \Gamma_{1 v}\right\rangle=\frac{i \hbar}{4 m_{0}^{2} c^{2}}\left\langle\Gamma_{1 v}\left|\frac{\partial V}{\partial z} p_{x}-\frac{\partial V}{\partial x} p_{z}\right| \Gamma_{5 v}^{x}\right\rangle, \\
& \Delta_{4}=\frac{i \hbar}{4 m_{0}^{2} c^{2}}\left\langle\Gamma_{5 v}^{y}\left|\frac{\partial V}{\partial y} p_{z}-\frac{\partial V}{\partial z} p_{y}\right| \Gamma_{1 c}\right\rangle=\frac{i \hbar}{4 m_{0}^{2} c^{2}}\left\langle\Gamma_{1 c}\left|\frac{\partial V}{\partial z} p_{x}-\frac{\partial V}{\partial x} p_{z}\right| \Gamma_{5 v}^{x}\right\rangle .
\end{aligned}
$$

Matrix representation of $H_{k p}^{(1)}$ :

$$
H_{k p}^{(1)}=\left[\begin{array}{cccccccc}
0 & 0 & i A_{7} k_{-} & 0 & 0 & 0 & -\frac{1}{\sqrt{2}} P_{2} k_{-} & 0 \\
0 & 0 & -i A_{7} k_{+} & 0 & 0 & 0 & \frac{1}{\sqrt{2}} P_{2} k_{+} & 0 \\
-i A_{7} k_{+} & i A_{7} k_{-} & 0 & 0 & 0 & 0 & P_{1} k_{z} & 0 \\
0 & 0 & 0 & 0 & 0 & -i A_{7} k_{+} & 0 & \frac{1}{\sqrt{2}} P_{2} k_{+} \\
0 & 0 & 0 & 0 & 0 & i A_{7} k_{-} & 0 & -\frac{1}{\sqrt{2}} P_{2} k_{-} \\
0 & 0 & 0 & i A_{7} k_{-} & -i A_{7} k_{+} & 0 & 0 & P_{1} k_{z} \\
-\frac{1}{\sqrt{2}} P_{2} k_{+} & \frac{1}{\sqrt{2}} P_{2} k_{-} & P_{1} k_{z} & 0 & 0 & 0 & 0 & 0 \\
0 & 0 & 0 & \frac{1}{\sqrt{2}} P_{2} k_{-} & -\frac{1}{\sqrt{2}} P_{2} k_{+} & P_{1} k_{z} & 0 & 0
\end{array}\right] \text {, }
$$

with the definitions

$$
\begin{aligned}
A_{7} & =\frac{i}{\sqrt{2}} \frac{\hbar}{m_{0}}\left\langle\Gamma_{5 v}^{x}\left|p_{x}\right| \Gamma_{1 v}\right\rangle=\frac{i}{\sqrt{2}} \frac{\hbar}{m_{0}}\left\langle\Gamma_{5 v}^{y}\left|p_{y}\right| \Gamma_{1 v}\right\rangle, \\
P_{2} & =i \frac{\hbar}{m_{0}}\left\langle\Gamma_{5 v}^{x}\left|p_{x}\right| \Gamma_{1 c}\right\rangle=i \frac{\hbar}{m_{0}}\left\langle\Gamma_{5 v}^{y}\left|p_{y}\right| \Gamma_{1 c}\right\rangle, \\
P_{1} & =i \frac{\hbar}{m_{0}}\left\langle\Gamma_{1 v}\left|p_{z}\right| \Gamma_{1 c}\right\rangle, \\
k_{ \pm} & =k_{x} \pm i k_{y} .
\end{aligned}
$$

Matrix representation of $H_{k S O}^{(1)}$ :

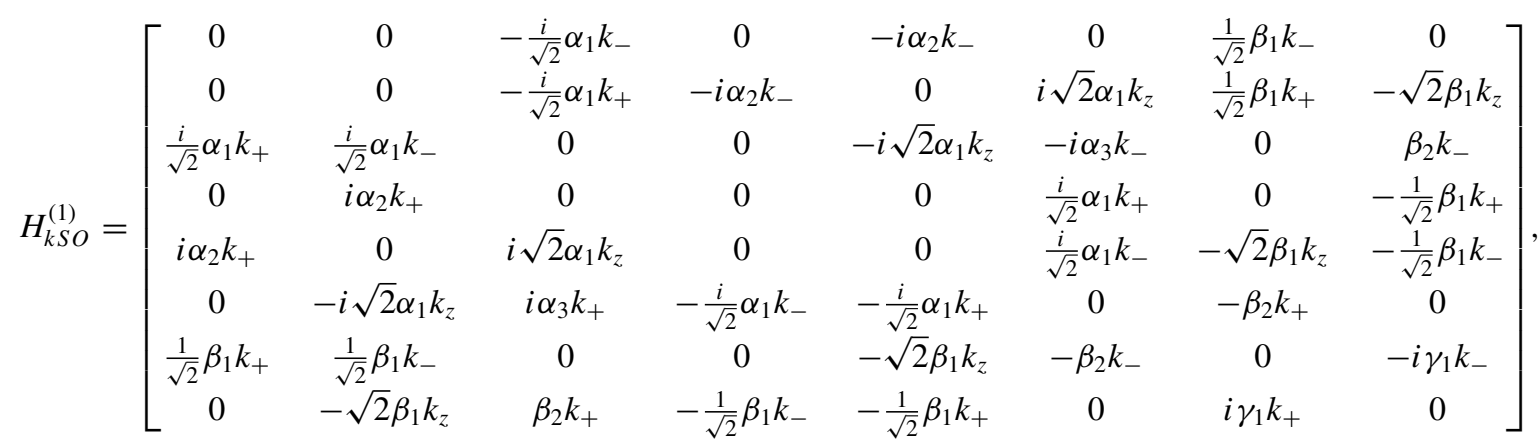


with the definitions

$$
\begin{aligned}
& \alpha_{1}=\frac{\hbar^{2}}{4 m_{0}^{2} c^{2}}\left\langle\Gamma_{5 v}^{x}\left|\frac{\partial V}{\partial x}\right| \Gamma_{1 v}\right\rangle=\frac{\hbar^{2}}{4 m_{0}^{2} c^{2}}\left\langle\Gamma_{5 v}^{y}\left|\frac{\partial V}{\partial y}\right| \Gamma_{1 v}\right\rangle, \\
& \alpha_{2}=\frac{\hbar^{2}}{4 m_{0}^{2} c^{2}}\left\langle\Gamma_{5 v}^{x}\left|\frac{\partial V}{\partial z}\right| \Gamma_{5 v}^{x}\right\rangle=\frac{\hbar^{2}}{4 m_{0}^{2} c^{2}}\left\langle\Gamma_{5 v}^{y}\left|\frac{\partial V}{\partial z}\right| \Gamma_{5 v}^{y}\right\rangle, \\
& \alpha_{3}=\frac{\hbar^{2}}{4 m_{0}^{2} c^{2}}\left\langle\Gamma_{1 v}\left|\frac{\partial V}{\partial z}\right| \Gamma_{1 v}\right\rangle, \\
& \beta_{1}=\frac{\hbar^{2}}{4 m_{0}^{2} c^{2}}\left\langle\Gamma_{5 v}^{x}\left|\frac{\partial V}{\partial x}\right| \Gamma_{1 c}\right\rangle=\frac{\hbar^{2}}{4 m_{0}^{2} c^{2}}\left\langle\Gamma_{5 v}^{y}\left|\frac{\partial V}{\partial y}\right| \Gamma_{1 c}\right\rangle, \\
& \beta_{2}=\frac{\hbar^{2}}{4 m_{0}^{2} c^{2}}\left\langle\Gamma_{1 v}\left|\frac{\partial V}{\partial z}\right| \Gamma_{1 c}\right\rangle, \\
& \gamma_{1}=\frac{\hbar^{2}}{4 m_{0}^{2} c^{2}}\left\langle\Gamma_{1 c}\left|\frac{\partial V}{\partial z}\right| \Gamma_{1 c}\right\rangle .
\end{aligned}
$$

Matrix representation of $H_{k p}^{(2)}$ :

$$
H_{k p}^{(2)}=\left[\begin{array}{cccccccc}
\lambda+\theta & -K^{*} & -H^{*} & 0 & 0 & 0 & T^{*} & 0 \\
-K & \lambda+\theta & H & 0 & 0 & 0 & T & 0 \\
-H & H^{*} & \lambda & 0 & 0 & 0 & U & 0 \\
0 & 0 & 0 & \lambda+\theta & -K & H & 0 & T \\
0 & 0 & 0 & -K^{*} & \lambda+\theta & -H^{*} & 0 & T^{*} \\
0 & 0 & 0 & H^{*} & -H & \lambda & 0 & U \\
T & T^{*} & U^{*} & 0 & 0 & 0 & V & 0 \\
0 & 0 & 0 & T^{*} & T & U^{*} & 0 & V
\end{array}\right],
$$

with elements given by

$$
\begin{aligned}
\lambda & =A_{1} k_{z}^{2}+A_{2}\left(k_{x}^{2}+k_{y}^{2}\right), \\
\theta & =A_{3} k_{z}^{2}+A_{4}\left(k_{x}^{2}+k_{y}^{2}\right), \\
K & =A_{5} k_{+}^{2}, \\
H & =A_{6} k_{+} k_{z}, \\
T & =i B_{3} k_{+} k_{z}, \\
U & =i\left[B_{1} k_{z}^{2}+B_{2}\left(k_{x}^{2}+k_{y}^{2}\right)\right], \\
V & =e_{1} k_{z}^{2}+e_{2}\left(k_{x}^{2}+k_{y}^{2}\right),
\end{aligned}
$$

and all the parameters in units of $\hbar^{2} / 2 m_{0}$.

The term $\mathbf{H}_{\mathbf{k} 2}$ is already included in the diagonal terms of $H_{k p}^{(2)}$. Strictly speaking, the matrix representation $H_{k p}^{(2)}$ is defined as

$$
H_{k p, \alpha \alpha^{\prime}}^{(2)}=\mathbf{H}_{\mathbf{k} 2} \delta_{\alpha \alpha^{\prime}}+\sum_{\beta}^{B} \frac{\left\langle\alpha\left|\mathbf{H}_{\mathbf{k p}}\right| \beta\right\rangle\left\langle\beta\left|\mathbf{H}_{\mathbf{k p}}\right| \alpha^{\prime}\right\rangle}{E_{\alpha \alpha^{\prime}}-E_{\beta}} .
$$

The second-order $k \cdot p$ parameters $A_{1}$ to $A_{6}$ are defined the same way as in Ref. [28], while $e_{1}, e_{2}, B_{1}, B_{2}$, and $B_{3}$ are given by

$$
e_{1}=1+\frac{2}{m_{0}} \sum_{\beta}^{B\left[\Gamma_{1}\right]} \frac{\left|\left\langle\Gamma_{1 c}\left|p_{z}\right| \beta\right\rangle\right|^{2}}{E_{1 c}-E_{\beta}}
$$

$$
\begin{aligned}
& e_{2}=1+\frac{2}{m_{0}} \sum_{\beta}^{B\left[\Gamma_{5}\right]} \frac{\left|\left\langle\Gamma_{1 c}\left|p_{x}\right| \beta\right\rangle\right|^{2}}{E_{1 c}-E_{\beta}} \\
& =1+\frac{2}{m_{0}} \sum_{\beta}^{B\left[\Gamma_{5}\right]} \frac{\left|\left\langle\Gamma_{1 c}\left|p_{y}\right| \beta\right\rangle\right|^{2}}{E_{1 c}-E_{\beta}}, \\
& B_{1}=\frac{2}{m_{0}} \sum_{\beta}^{B\left[\Gamma_{1}\right]} \frac{\left\langle\Gamma_{1 v}\left|p_{z}\right| \beta\right\rangle\left\langle\beta\left|p_{z}\right| \Gamma_{1 c}\right\rangle}{E_{1 v 1 c}-E_{\beta}}, \\
& B_{2}=\frac{2}{m_{0}} \sum_{\beta}^{B\left[\Gamma_{5}\right]} \frac{\left\langle\Gamma_{1 v}\left|p_{x}\right| \beta\right\rangle\left\langle\beta\left|p_{x}\right| \Gamma_{1 c}\right\rangle}{E_{1 v 1 c}-E_{\beta}} \\
& =\frac{2}{m_{0}} \sum_{\beta}^{B\left[\Gamma_{5}\right]} \frac{\left\langle\Gamma_{1 v}\left|p_{y}\right| \beta\right\rangle\left\langle\beta\left|p_{y}\right| \Gamma_{1 c}\right\rangle}{E_{1 v 1 c}-E_{\beta}}, \\
& B_{3}=\frac{\sqrt{2}}{m_{0}}\left(\sum_{\beta}^{B\left[\Gamma_{1}\right]} \frac{\left\langle\Gamma_{5 v}^{x}\left|p_{x}\right| \beta\right\rangle\left\langle\beta\left|p_{z}\right| \Gamma_{1 c}\right\rangle}{E_{5 v 1 c}-E_{\beta}}\right. \\
& \left.+\sum_{\beta}^{B\left[\Gamma_{5}\right]} \frac{\left\langle\Gamma_{5 v}^{x}\left|p_{z}\right| \beta\right\rangle\left\langle\beta\left|p_{x}\right| \Gamma_{1 c}\right\rangle}{E_{5 v 1 c}-E_{\beta}}\right) \\
& =\frac{\sqrt{2}}{m_{0}}\left(\sum_{\beta}^{B\left[\Gamma_{1}\right]} \frac{\left\langle\Gamma_{5 v}^{y}\left|p_{y}\right| \beta\right\rangle\left\langle\beta\left|p_{z}\right| \Gamma_{1 c}\right\rangle}{E_{5 v 1 c}-E_{\beta}}\right. \\
& \left.+\sum_{\beta}^{B\left[\Gamma_{5}\right]} \frac{\left\langle\Gamma_{5 v}^{y}\left|p_{z}\right| \beta\right\rangle\left\langle\beta\left|p_{y}\right| \Gamma_{1 c}\right\rangle}{E_{5 v 1 c}-E_{\beta}}\right),
\end{aligned}
$$




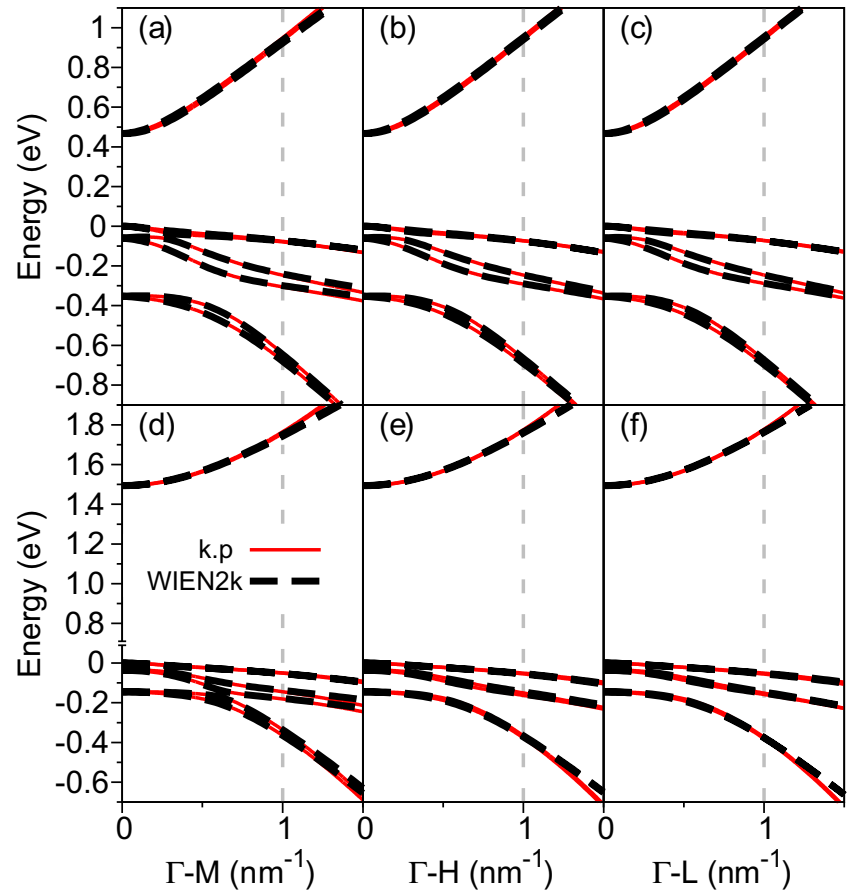

FIG. 8. Comparison of band structures for (a) $\Gamma-\mathrm{M}$, (b) $\Gamma-\mathrm{H}$, and (c) $\Gamma$-L directions of InAs and (d) $\Gamma-\mathrm{M}$, (e) $\Gamma-\mathrm{H}$, and (f) $\Gamma-\mathrm{L}$ directions of InP. The line schemes follow Fig. 4.

with nonzero contributions represented by the irreducible representations in the brackets above the summation.

\section{APPENDIX C: FITTING IN OTHER DIRECTIONS}

The comparison between the fitted and WIEN2k ab initio band structures is displayed in Fig. 8 for the $\Gamma-\mathrm{M}, \Gamma-\mathrm{H}$, and $\Gamma-\mathrm{L}$ directions. For the $\Gamma$-M direction, we have the same behavior discussed for $\Gamma-\mathrm{K}$. However, the $k \cdot p$ band structures in the $\Gamma-\mathrm{H}$ and $\Gamma$-L directions have closer values to $a b$ initio. This better agreement arises from the second-order parameters $A_{6}$ and $B_{3}$ which only couple the $k_{x} k_{y}$ plane to $k_{z}$, providing additional corrections to the band structures.

In Fig. 9 and Fig. 10, we show the comparison of spin splittings along the $\Gamma-\mathrm{M}, \Gamma-\mathrm{H}$, and $\Gamma-\mathrm{L}$ directions for InAs and InP, respectively. For the $\Gamma-\mathrm{H}$ and $\Gamma$-L directions, the spin splittings are usually smaller compared to $\Gamma-\mathrm{K}$ and $\Gamma-\mathrm{M}$. This difference, however, depends on the material and the energy band. For instance, $\mathrm{CH}$ and $\mathrm{LH}$ values for InAs in $\Gamma-\mathrm{H}$ and $\Gamma-\mathrm{L}$ are approximately half the value in the $\Gamma-\mathrm{K}$ and $\Gamma-\mathrm{M}$ directions while $\mathrm{LH}$ and $\mathrm{CH}$ values for $\mathrm{InP}$ are approximately one-fourth of the values. Because of these larger differences for InP, the spin splittings for $\mathrm{CH}$ bands along $\Gamma-\mathrm{H}$ and $\Gamma-\mathrm{L}$ show a small deviation compared to ab initio [Figs. 10(h) and 10(1)]. However, the crossings for HH bands [Figs. 10(f) and 10(j)] are precisely reproduced. Comparing all directions, we verify that our $k \cdot p$ model and parameter sets reproduce with great agreement the $a b$ initio band structure and spin splittings along all the considered directions of the FBZ.

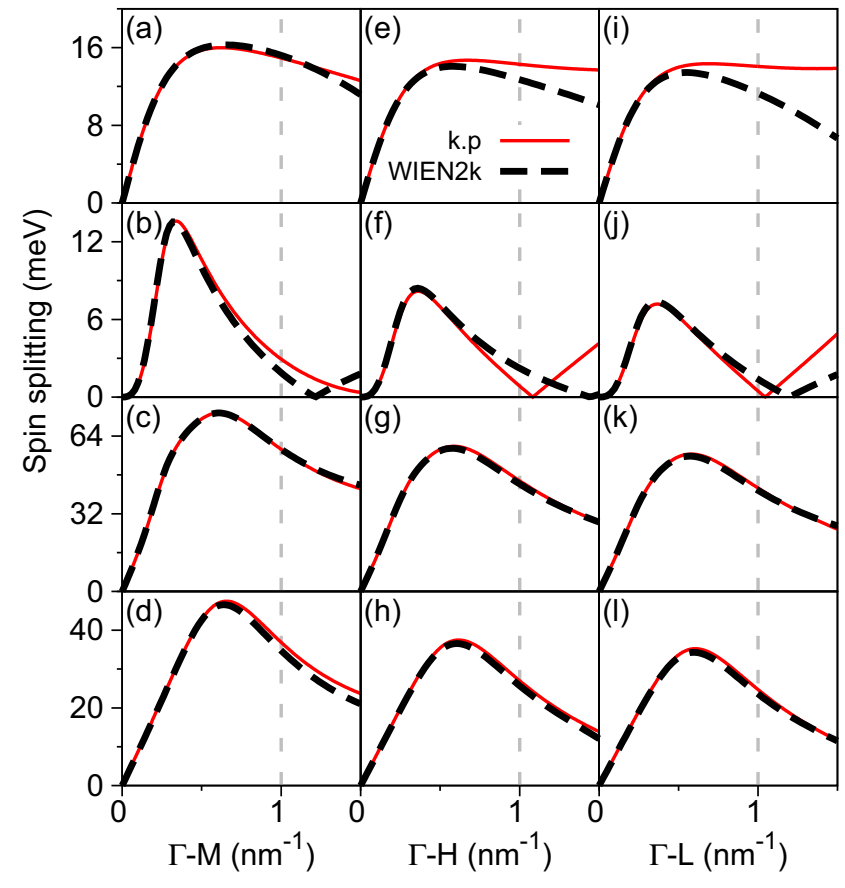

FIG. 9. Comparison of the InAs spin splittings for (a), (e), (i) CB; (b), (f), (j) HH; (c), (g), (k) CH; and (d), (h), (l) LH along $\Gamma-\mathrm{M}, \Gamma-\mathrm{H}$, and $\Gamma$-L directions. The line schemes follow Fig. 4.

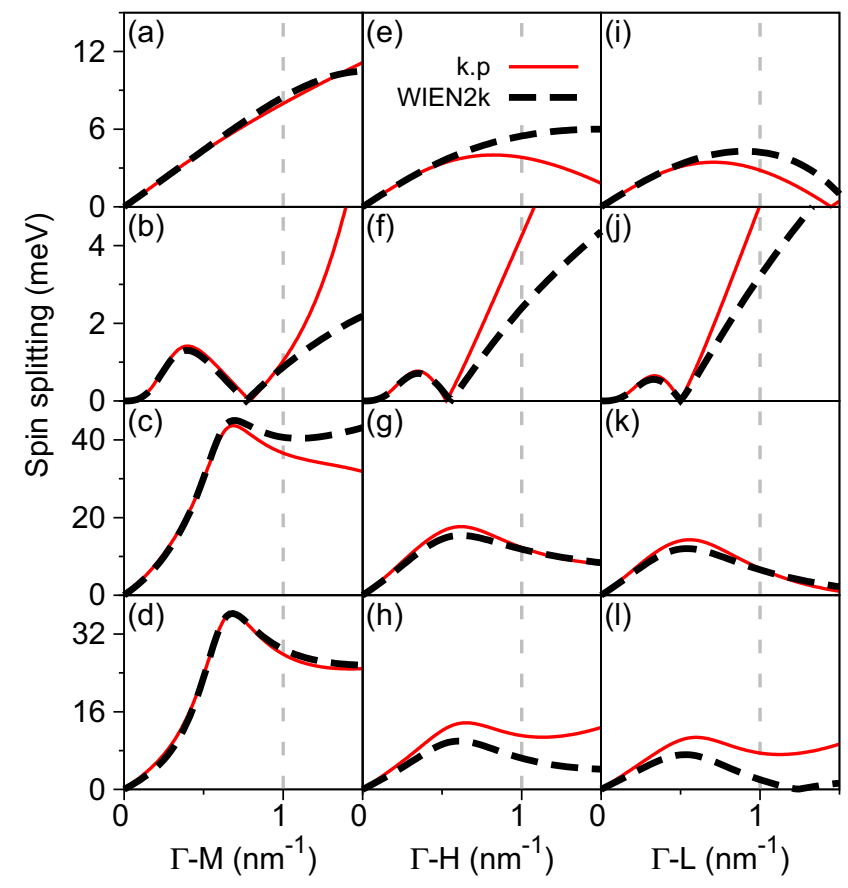

FIG. 10. Comparison of the InP spin splittings for (a), (e), (i) CB (b), (f), (j) HH; (c), (g), (k) LH; and (d), (h), (l) $\mathrm{CH}$ along $\Gamma-\mathrm{M}, \Gamma-\mathrm{H}$, and $\Gamma$-L directions. The line schemes follow Fig. 4. 
[1] Y. Li, F. Qian, J. Xiang, and C. M. Lieber, Mater. Today 9, 18 (2006).

[2] P. Caroff, K. A. Dick, J. Johansson, M. E. Messing, K. Deppert, and L. Samuelson, Nat. Nanotechnol. 4, 50 (2009).

[3] T. T. T. Vu, T. Zehender, M. A. Verheijen, S. R. Plissard, G. W. G. Immink, J. E. M. Haverkort, and E. P. A. M. Bakkers, Nanotechnology 24, 115705 (2013).

[4] D. Pan, M. Fu, X. Yu, X. Wang, L. Zhu, S. Nie, S. Wang, Q. Chen, P. Xiong, S. von Molnár, and J. Zhao, Nano Lett. 14, 1214 (2014).

[5] K. A. Dick, C. Thelander, L. Samuelson, and P. Caroff, Nano Lett. 10, 3494 (2010).

[6] J. Bolinsson, P. Caroff, B. Mandl, and K. A. Dick, Nanotechnology 22, 265606 (2011).

[7] S. Lehmann, J. Wallentin, D. Jacobsson, K. Deppert, and K. A. Dick, Nano Lett. 13, 4099 (2013).

[8] T. Ba Hoang, A. F. Moses, L. Ahtapodov, H. Zhou, D. L. Dheeraj, A. T. J. van Helvoort, B.-O. Fimland, and H. Weman, Nano Lett. 10, 2927 (2010).

[9] E. G. Gadret, G. O. Dias, L. C. O. Dacal, M. M. de Lima, C. V. R. S. Ruffo, F. Iikawa, M. J. S. P. Brasil, T. Chiaramonte, M. A. Cotta, L. H. G. Tizei, D. Ugarte, and A. Cantarero, Phys. Rev. B 82, 125327 (2010).

[10] P. E. Faria Junior, T. Campos, and G. M. Sipahi, J. Appl. Phys. 116, 193501 (2014).

[11] C. Thelander, P. Caroff, S. Plissard, A. Dey, and K. Dick, Nano Lett. 11, 2424 (2011).

[12] A. Konar, J. Mathew, K. Nayak, M. Bajaj, R. K. Pandey, S. Dhara, K. V. R. M. Murali, and M. M. Deshmukh, Nano Lett. 15, 1684 (2015).

[13] J. Li, X. Yan, F. Sun, X. Zhang, and X. Ren, Appl. Phys. Lett. 107, 263103 (2015).

[14] P. Chuang, S.-C. Ho, L. W. Smith, F. Sfigakis, M. Pepper, C.-H. Chen, J.-C. Fan, J. P. Griffiths, I. Farrer, H. E. Beere, G. A. C. Jones, D. A. Ritchie, and T.-M. Chen, Nat. Nanotechnol. 10, 35 (2014).

[15] A. Das, Y. Ronen, Y. Most, Y. Oreg, M. Heiblum, and H. Shtrikman, Nat. Phys. 8, 887 (2012).

[16] X. Li, X. Wei, T. Xu, D. Pan, J. Zhao, and Q. Chen, Adv. Mater. 27, 2852 (2015).

[17] H. J. Joyce, C. J. Docherty, Q. Gao, H. H. Tan, C. Jagadish, J. Lloyd-Hughes, L. M. Herz, and M. B. Johnston, Nanotechnology 24, 214006 (2013).

[18] Y. Cui, J. Wang, S. R. Plissard, A. Cavalli, T. T. T. Vu, R. P. J. van Veldhoven, L. Gao, M. Trainor, M. A. Verheijen, J. E. M. Haverkort, and E. P. A. M. Bakkers, Nano Lett. 13, 4113 (2013).

[19] Z. Wang, B. Tian, M. Paladugu, M. Pantouvaki, N. Le Thomas, C. Merckling, W. Guo, J. Dekoster, J. V. Campenhout, P. Absil, and D. V. Thourhout, Nano Lett. 13, 5063 (2013).

[20] S. F. Svensson, S. Jeppesen, C. Thelander, L. Samuelson, H. Linke, and K. A. Dick, Nanotechnology 24, 345601 (2013).

[21] D. Lindgren, K. Kawaguchi, M. Heurlin, M. T. Borgström, M.-E. Pistol, L. Samuelson, and A. Gustafsson, Nanotechnology 24, 225203 (2013).

[22] Z. Zanolli, F. Fuchs, J. Furthmüller, U. von Barth, and F. Bechstedt, Phys. Rev. B 75, 245121 (2007).

[23] A. De and C. E. Pryor, Phys. Rev. B 81, 155210 (2010).

[24] L. C. O. Dacal and A. Cantarero, Solid State Commun. 151, 781 (2011).
[25] A. Belabbes, C. Panse, J. Furthmüller, and F. Bechstedt, Phys. Rev. B 86, 075208 (2012).

[26] C. Hajlaoui, L. Pedesseau, F. Raouafi, F. Ben CheikhLarbi, J. Even, and J.-M. Jancu, J. Phys. D: Appl. Phys. 46, 505106 (2013).

[27] L. C. O. Dacal and A. Cantarero, Mater. Res. Express 1, 015702 (2014).

[28] S. L. Chuang and C. S. Chang, Phys. Rev. B 54, 2491 (1996).

[29] P. Rinke, M. Winkelnkemper, A. Qteish, D. Bimberg, J. Neugebauer, and M. Scheffler, Phys. Rev. B 77, 075202 (2008).

[30] P. E. Faria Junior and G. M. Sipahi, J. Appl. Phys. 112, 103716 (2012).

[31] T. Cheiwchanchamnangij and W. R. Lambrecht, Phys. Rev. B 84, 035203 (2011).

[32] P. Blaha, K. Schwarz, G. K. H. Madsen, D. Kvasnicka, and J. Luitz, WIEN2k, An Augmented Plane Wave + Local Orbitals Program for Calculating Crystal Properties (Vienna University of Technology, Vienna, 2013).

[33] I. Žutić, J. Fabian, and S. Das Sarma, Rev. Mod. Phys. 76, 323 (2004).

[34] J. Hohenberg and W. Kohn, Phys. Rev. 136, B864 (1964).

[35] A. D. Becke and E. R. Johnson, J. Chem. Phys. 124, 221101 (2006).

[36] F. Tran and P. Blaha, Phys. Rev. Lett. 102, 226401 (2009).

[37] Y.-S. Kim, M. Marsman, G. Kresse, F. Tran, and P. Blaha, Phys. Rev. B 82, 205212 (2010).

[38] D. Koller, F. Tran, and P. Blaha, Phys. Rev. B 83, 195134 (2011).

[39] D. Koller, F. Tran, and P. Blaha, Phys. Rev. B 85, 155109 (2012).

[40] Y.-S. Kim, K. Hummer, and G. Kresse, Phys. Rev. B 80, 035203 (2009).

[41] A. N. Chantis, M. van Schilfgaarde, and T. Kotani, Phys. Rev. Lett. 96, 086405 (2006).

[42] J.-W. Luo, G. Bester, and A. Zunger, Phys. Rev. Lett. 102, 056405 (2009).

[43] A. N. Chantis, N. E. Christensen, A. Svane, and M. Cardona, Phys. Rev. B 81, 205205 (2010).

[44] J. P. Perdew and A. Zunger, Phys. Rev. B 23, 5048 (1981).

[45] J. P. Perdew, K. Burke, and M. Ernzerhof, Phys. Rev. Lett. 77, 3865 (1996).

[46] D. J. Singh and L. Nordstrom, Planewaves, Pseudopotentials, and the LAPW Method (Springer, Berlin, 2006).

[47] M. Gmitra and J. Fabian, arXiv:1606.00588.

[48] M. S. Dresselhaus, G. Dresselhaus, and A. Jorio, Group Theory: Application to the Physics of Condensed Matter, 1st ed. (Springer, Berlin, 2008). From Table D.11, replace $\Delta_{1 \ldots 6}$ with $\Gamma_{1 \ldots 6}$ and use the factor $T_{\Delta}=1$.

[49] M. Cardona, N. E. Christensen, and G. Fasol, Phys. Rev. B 38, 1806 (1988)

[50] P. Käckell, B. Wenzien, and F. Bechstedt, Phys. Rev. B 50, 17037 (1994).

[51] M. I. McMahon and R. J. Nelmes, Phys. Rev. Lett. 95, 215505 (2005).

[52] D. Kriegner, C. Panse, B. Mandl, K. A. Dick, M. Keplinger, J. M. Persson, P. Caroff, D. Ercolani, L. Sorba, F. Bechstedt, J. Stangl, and G. Bauer, Nano Lett. 11, 1483 (2011).

[53] C. Panse, D. Kriegner, and F. Bechstedt, Phys. Rev. B 84, 075217 (2011).

[54] R. C. Casella, Phys. Rev. 114, 1514 (1959).

[55] J. J. Hopfield, J. Appl. Phys. 32, 2277 (1961). 
[56] M. De Luca, A. Zilli, A. Fonseka, S. Mokkapati, A. Miriametro, H. Tan, L. Smith, C. Jagadish, M. Capizzi, and A. Polimeni, Nano Lett. 15, 998 (2015).

[57] A. Zilli, M. De Luca, D. Tedeschi, A. Fonseka, A. Miriametro, H. Tan, C. Jagadish, M. Capizzi, and A. Polimeni, ACS Nano 9, 4277 (2015).

[58] R. Enderlein and N. J. Horing, Fundamentals of Semiconductor Physics and Devices, 1st ed. (World Scientific Publishing, Singapore, 1997).

[59] P. O. Löwdin, J. Chem. Phys. 19, 1396 (1951).

[60] J. Bao, D. C. Bell, F. Capasso, N. Erdman, D. Wei, L. Fröberg, T. Mårtensson, and L. Samuelson, Adv. Mater. 21, 3654 (2009).

[61] G. Koblmüller, S. Hertenberger, K. Vizbaras, M. Bichler, F. Bao, J-P. Zhang, and G. Abstreiter, Nanotechnology 21, 365602 (2010).

[62] M. Möller, M. M. de Lima Jr., A. Cantarero, T. Chiaramonte, M. A. Cotta, and F. Iikawa, Nanotechnology 23, 375704 (2012).

[63] A. Mishra, L. V. Titova, T. B. Hoang, H. E. Jackson, L. M. Smith, J. M. Yarrison-Rice, Y. Kim, H. J. Joyce, Q. Gao, H. H. Tan, and C. Jagadish, Appl. Phys. Lett. 91, 263104 (2007).

[64] G. L. Tuin, M. T. Borgström, J. Trägårdh, M. Ek, L. R. Wallenberg, L. Samuelson, and M.-E. Pistol, Nano Res. 4, 159 (2011).

[65] As far as we are concerned, the only paper in the literature that provides a double-group description of the $k \cdot p$ model (for cubic crystals) is W. J. Elder, R. M. Ward, and J. Zhang, Phys. Rev. B 83, 165210 (2011).

[66] Explicitly, $u_{n, \Gamma}(\vec{r})=\left\langle\vec{r} \mid c_{n}\right\rangle$, with $n=1, \ldots, 8$ running over the energy bands at the $\Gamma$ point we are describing.

[67] R. Beresford, J. Appl. Phys. 95, 6216 (2004).

[68] J. Y. Fu and M. W. Wu, J. Appl. Phys. 104, 093712 (2008).
[69] A. Marnetto, M. Penna, and M. Goano, J. Appl. Phys. 108, 033701 (2010).

[70] M. S. Miao, Q. Yan, C. G. Van de Walle, W. K. Lou, L. L. Li, and K. Chang, Phys. Rev. Lett. 109, 186803 (2012).

[71] G. Dresselhaus, Phys. Rev. 100, 580 (1955).

[72] A. Punya and W. R. L. Lambrecht, Phys. Rev. B 85, 195147 (2012).

[73] M. Newville, T. Stensitzki, D. B. Allen, and A. Ingargiola, LMFIT: Non-linear least-square minimization and curve-fitting for Python (2014), doi:10.5281/zenodo.11813.

[74] The residue calculated for a specific direction in the FBZ is given by $r=\frac{1}{N} \sum_{n, \vec{k}}\left[f_{n}(\vec{k})-t_{n}(\vec{k})\right]^{2}$, in which $n$ runs over the 8 energy bands or 4 spin splitting curves, $\vec{k}$ runs over the $k$ points, $f_{n}(\vec{k})$ is the fitted data, $t_{n}(\vec{k})$ is the ab initio data, and $N$ is a normalization constant which includes the number of $k$ points used multiplied by the size of $n$. The total residue is the sum of the residues in all directions.

[75] S. L. Chuang and C. S. Chang, Appl. Phys. Lett. 68, 1657 (1996).

[76] See Supplemental Material at http://link.aps.org/supplemental/ 10.1103/PhysRevB.93.235204 for the curve fitting of the carrier density, the comparison between $a b$ initio and the analytical approach for conduction band and the fitting of the $6 \times 6$ Hamiltonian approach for valence band.

[77] J. I. Climente, C. Segarra, F. Rajadell, and J. Planelles, J. Appl. Phys. 119, 125705 (2016).

[78] M. W. Larsson, J. B. Wagner, M. Wallin, P. Håkansson, L. E. Fröberg, L. Samuelson, and L. R. Wallenberg, Nanotechnology 18, 015504 (2007).

[79] F. Boxberg, N. Søndergaard, and H. Q. Xu, Adv. Mater. 24, 4692 (2012).

[80] C. Hajlaoui, L. Pedesseau, F. Raouafi, F. Ben Cheikh Larbi, J. Even, and J.-M. Jancu, J. Exp. Theor. Phys. 121, 246 (2015). 


\title{
Supplemental material for the paper "Realistic multiband k.p approach from $a b$ initio and spin-orbit coupling effects of InAs and InP in wurtzite phase"
}

\author{
Paulo E. Faria Junior, ${ }^{1}$ Tiago Campos, ${ }^{1,2}$ Carlos M. O. Bastos, ${ }^{1}$ \\ Martin Gmitra, ${ }^{2}$ Jaroslav Fabian, ${ }^{2}$ and Guilherme M. Sipahi ${ }^{1,3}$ \\ ${ }^{1}$ São Carlos Institute of Physics, University of São Paulo, 13566-590 São Carlos, São Paulo, Brazil \\ ${ }^{2}$ Institute for Theoretical Physics, University of Regensburg, 93040 Regensburg, Germany \\ ${ }^{3}$ Department of Physics, State University of New York at Buffalo, Buffalo, New York 14260, USA
}

\section{CURVE FITTING FOR THE CARRIER DENSITY}

In this section, we provide a curve fitting of the carrier densities presented in Figs. $7(\mathrm{c})$ and $7(\mathrm{~d})$ of the main paper. The 3D parabolic model gives us a carrier density dependence of the form $n(E) \propto E^{\frac{3}{2}}$, with the proportionality constant dependent on the effective mass. Since the calculated band structures of InAs and InP do not obey this parabolic behavior, we use the functional form

$$
n(E)=a+b E+c E^{d}
$$

to fit the carrier density as a function of the Fermi energy, with $E=E_{f}-E_{g}$ for the electrons and $E=\left|E_{f}\right|$ for the holes. The energy $E$ is given in meV and the carrier density $n$ in $10^{16} \mathrm{~cm}^{-3}$. This functional form does not carry any physical meaning in its different terms, it just provides an analytical way to predict the carrier density given the Fermi energy measured from the band edge. Because of linear SOC terms in conduction band, DOS is nonzero at the energy gap, therefore the requirement to use the fitting parameter $a$. Also, the linear dispersion $b E$ gives a better agreement with the numerical data. For valence band, the fitting of parameters $c$ and $d$ is enough, and therefore $a=b=0$. The best fitting is obtained by separating the carrier density in two different regions, which we call low and high energy regimes. The fitting parameters are shown in table I.

Table I: Numerical parameters to be used in equation (1) to predict the carrier density or the Fermi energy.

\begin{tabular}{lcccc|lcc}
\hline \hline \multicolumn{3}{c|}{ Electrons } & \multicolumn{2}{c}{ Holes } \\
\hline & $a$ & $b$ & $c$ & $d$ & & $c$ & $d$ \\
\hline InAs low $(E \leq 35)$ & 0.0385 & 0.1115 & 0.0684 & 1.6417 & InAs low $(E \leq 6)$ & 1.1076 & 1.5923 \\
InAs high $(35<E \leq 300)$ & -9.4227 & 0.8434 & 0.0023 & 2.2664 & InAs high $(6<E \leq 100)$ & 0.4065 & 2.1492 \\
\hline InP low $(E \leq 35)$ & 0.0117 & 0.1022 & 0.5636 & 1.5239 & InP low $(E \leq 10)$ & 2.4625 & 1.6694 \\
InP high $(35<E \leq 300)$ & -10.1466 & 1.5372 & 0.2265 & 1.6747 & InP high $(10<E \leq 100)$ & 0.9292 & 2.0845 \\
\hline
\end{tabular}

\section{ANALYTICAL DESCRIPTION FOR THE CONDUCTION BAND COMPARED TO}

Using the analytical expressions for the conduction band provided in the main paper (section V), we show the comparison between this approach and the $a b$ initio data in Fig. 1. Although the description for InP provides better results further away of $\Gamma$ point, the maximum accurate energy is around $100 \mathrm{meV}$ above the gap for both materials.

\section{III. $6 \times 6$ MODEL FOR THE VALENCE BAND COMPARED TO AB INITIO}

\section{A. InAs}

Applying the fitting approach discussed in Sec. IV of the main paper for the valence band of InAs, we obtain the band structure displayed in Fig, 2. In order to achieve the monotonic behavior of the valence band, i. e., decreasing energy while increasing $k$, the resulting fitted parameters provide a band structure that is shifted to higher $k$ values. These same trends can be seen in the spin splitting, shown in Fig, 3, by looking at the peak values. The correct fitting closer to $\Gamma$ point does not provide the correct monotonic behavior, i. e., it either makes the spin splitting branches diverge drastically or the $\mathrm{HH}$ band acquires an upward curvature. Because of the small energy gap of InAs and the large SOC effects, we emphasize that including the explicit coupling with the conduction band is necessary and, therefore, the most suitable approach is the $8 \times 8$ model we discuss in the main paper. 

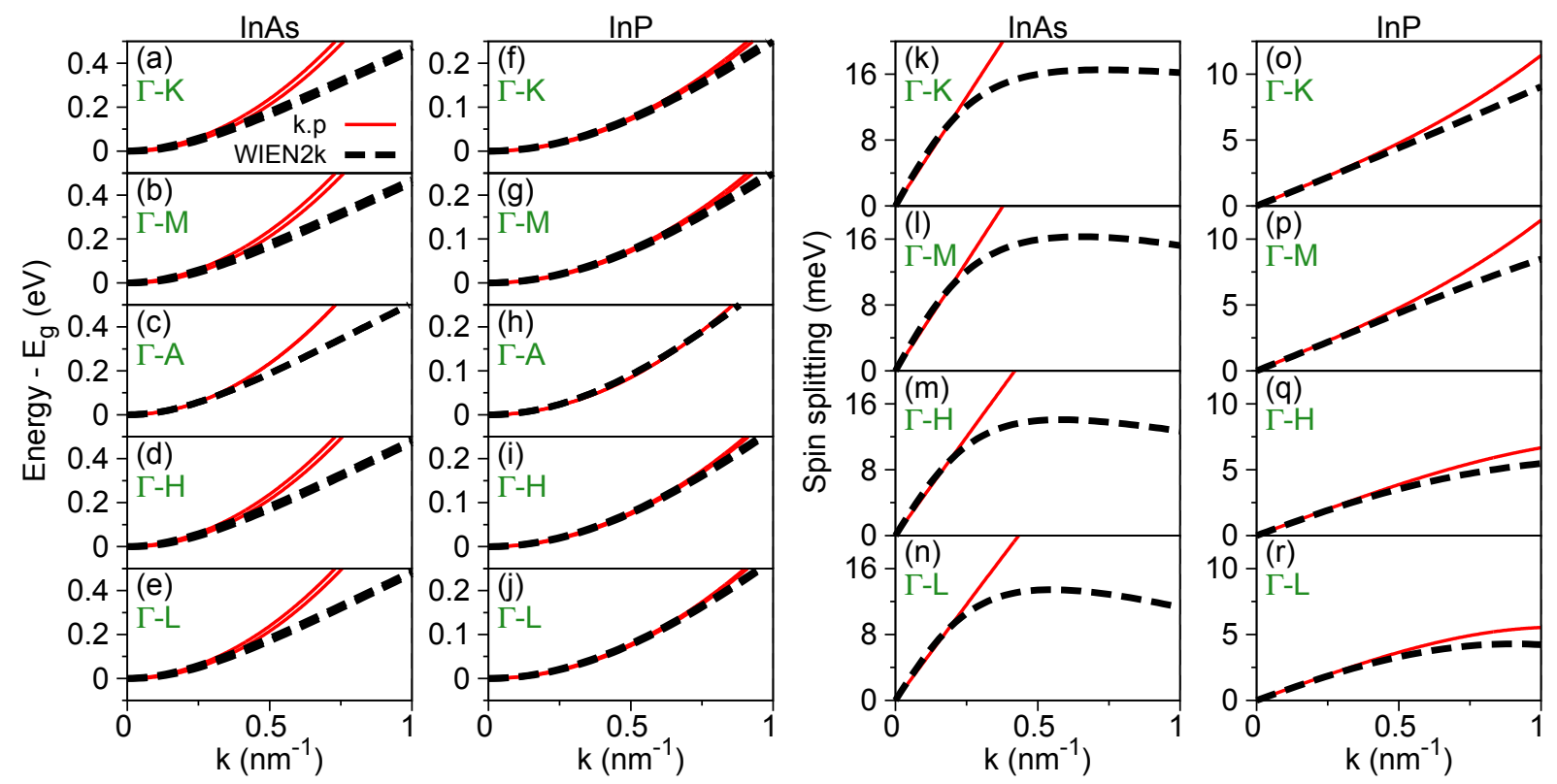

Figure 1: (Color online) Comparison between the analytical expression for conduction band and WIEN2k band structure for (a-e) InAs and (f-j) InP for $\Gamma-(K, M, A, H, L)$ directions. Comparison between the analytical spin splittings for (k-n) InAs and (o-r) InP along $\Gamma-(\mathrm{K}, \mathrm{M}, \mathrm{H}, \mathrm{L})$ directions.

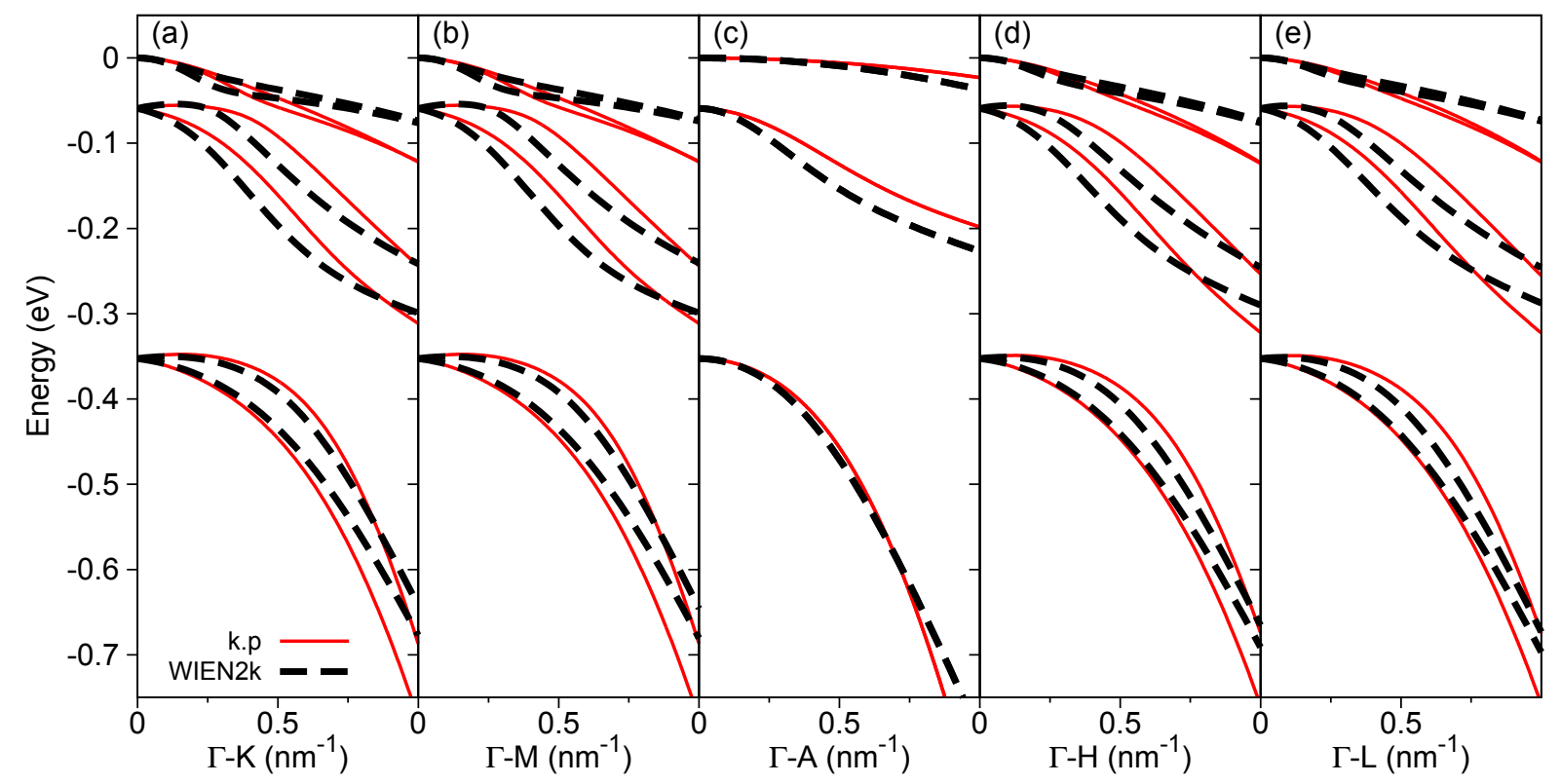

Figure 2: (Color online) Comparison between the $6 \times 6 \mathrm{k} . \mathrm{p}$ model for valence band and WIEN2k band structure for InAs along (a) $\Gamma-\mathrm{K}$, (b) $\Gamma-\mathrm{M},($ c) $\Gamma-\mathrm{A}$, (d) $\Gamma-\mathrm{H}$ and (e) $\Gamma$-L.

In Fig. 4 we show the spin expectation value in $y$ direction, $\left\langle\sigma_{y}\right\rangle$, along $\Gamma$-K direction for InAs valence band. Despite the differences for the band structure and the spin splittings, the spin orientation follows the same trends and signs of the $8 \times 8$ model, except for the crossing in the $\mathrm{HH}$ band that happens for a smaller $k$ value.

B. InP

For InP, the fitting approach for the valence band provides a reasonable agreement for the band structure, Fig. 5, and the spin splitting, Fig. 6. It is important to note that within this $6 \times 6$ model, there is only one parameter, $A_{6}$, that couples $k_{x(y)}$ and $k_{z}$ wave vectors. Because $\mathrm{InP}$ band structure along $\Gamma-\mathrm{H}(\mathrm{L})$ direcion is different from $\Gamma-\mathrm{K}(\mathrm{M})$ direction, only $A_{6}$ is not capable of correcting this anisotropy. Therefore, we see larger 


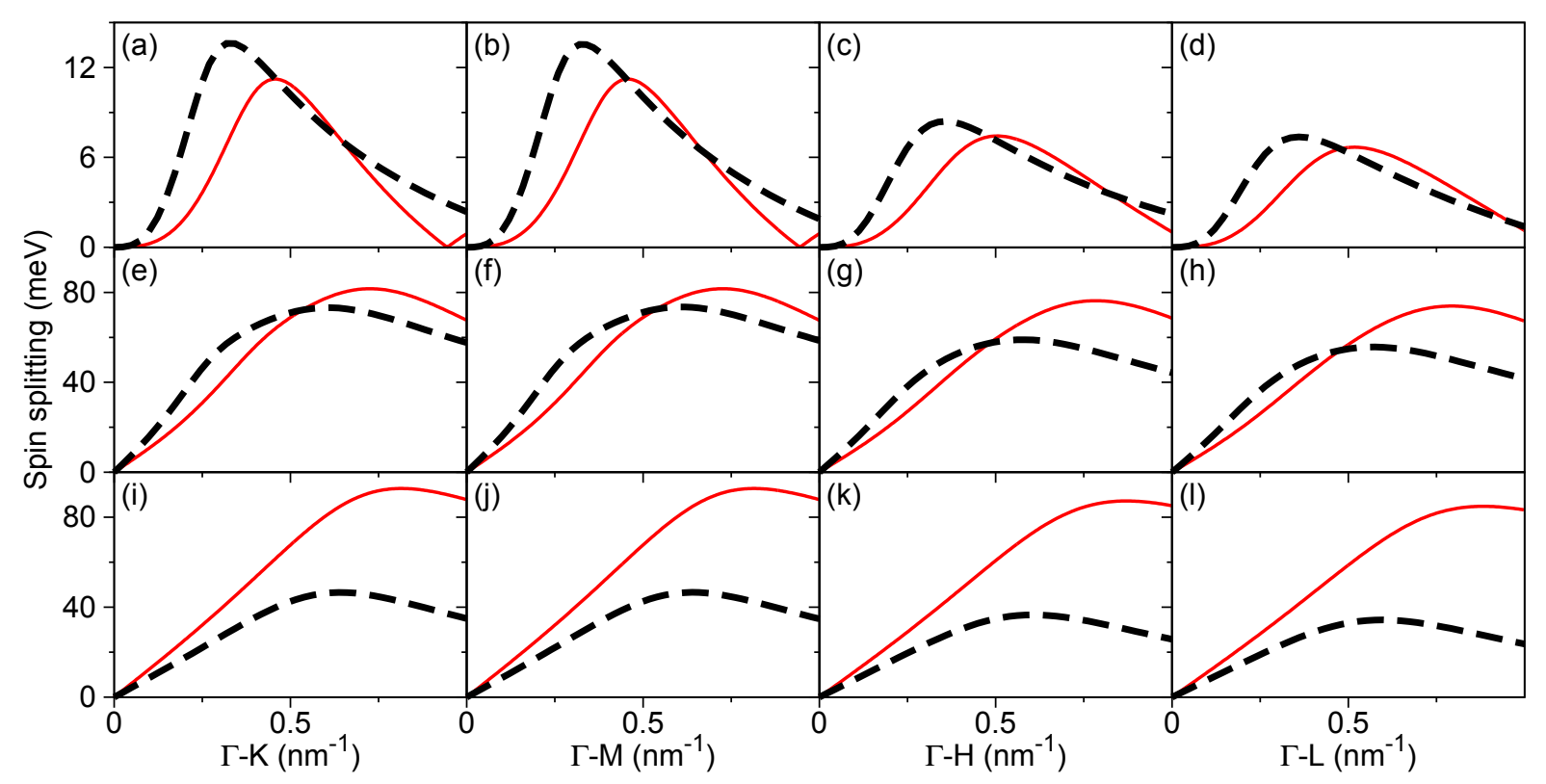

Figure 3: (Color online) Comparison of the InAs spin splittings for (a-d) HH, (e-h) CH and (i-l) LH along $\mathrm{C}-(\mathrm{K}, \mathrm{M}, \mathrm{H}$, L) direcions.
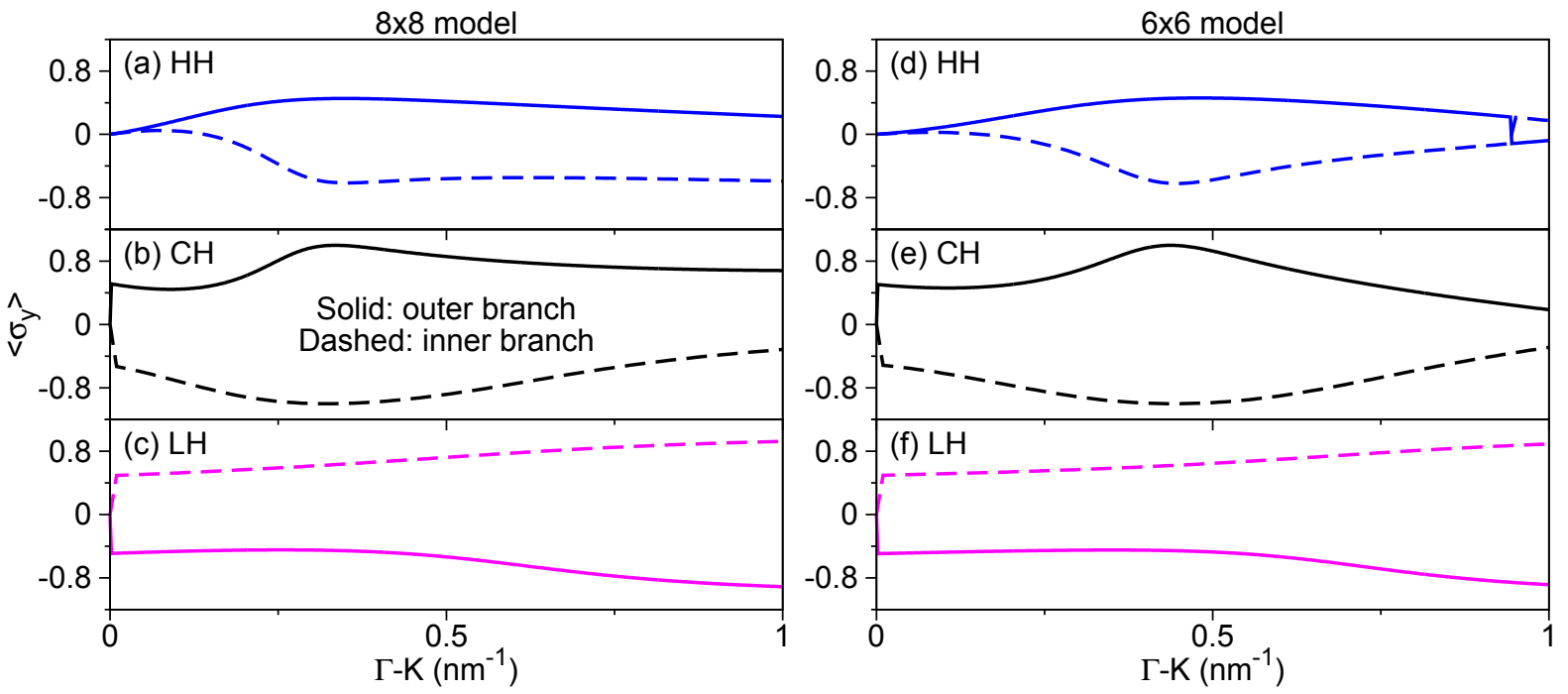

Figure 4: (Color online) Spin expectation value in $y$ direction, $\left\langle\sigma_{y}\right\rangle$, along $\Gamma$-K for the (a-c) $8 \times 8$ and (d-f) $6 \times 6$ model for $\mathrm{HH}, \mathrm{CH}$ and LH bands. The values of $\left\langle\sigma_{x}\right\rangle$ and $\left\langle\sigma_{z}\right\rangle$ are zero along $\Gamma-\mathrm{K}$. Solid (dashed) lines indicate the outer (inner) branch of the bands, as presented in Fig. 2(a) and 2(b) of the main paper.

differences for the band structure and spin splittings along $\Gamma$ - $H$ and $\Gamma$ - $\mathrm{L}$ directions.

In Fig. 7 we present $\left\langle\sigma_{y}\right\rangle$ along $\Gamma-\mathrm{K}$ direction for the valence band of InP. Although $\mathrm{HH}$ band in the $6 \times 6$ model shows a similar behavior, $\mathrm{LH}$ and $\mathrm{CH}$ bands do not follow the trends from the $8 \times 8$ approach. The correct description of InP spin texture, by imposing $A_{7}>\alpha_{1}$ in the fitting process (this is seen for InAs parameters), drastically compromises the band structure and spin splittings. Therefore, we also suggest the use of $8 \times 8$ model to treat InP. 


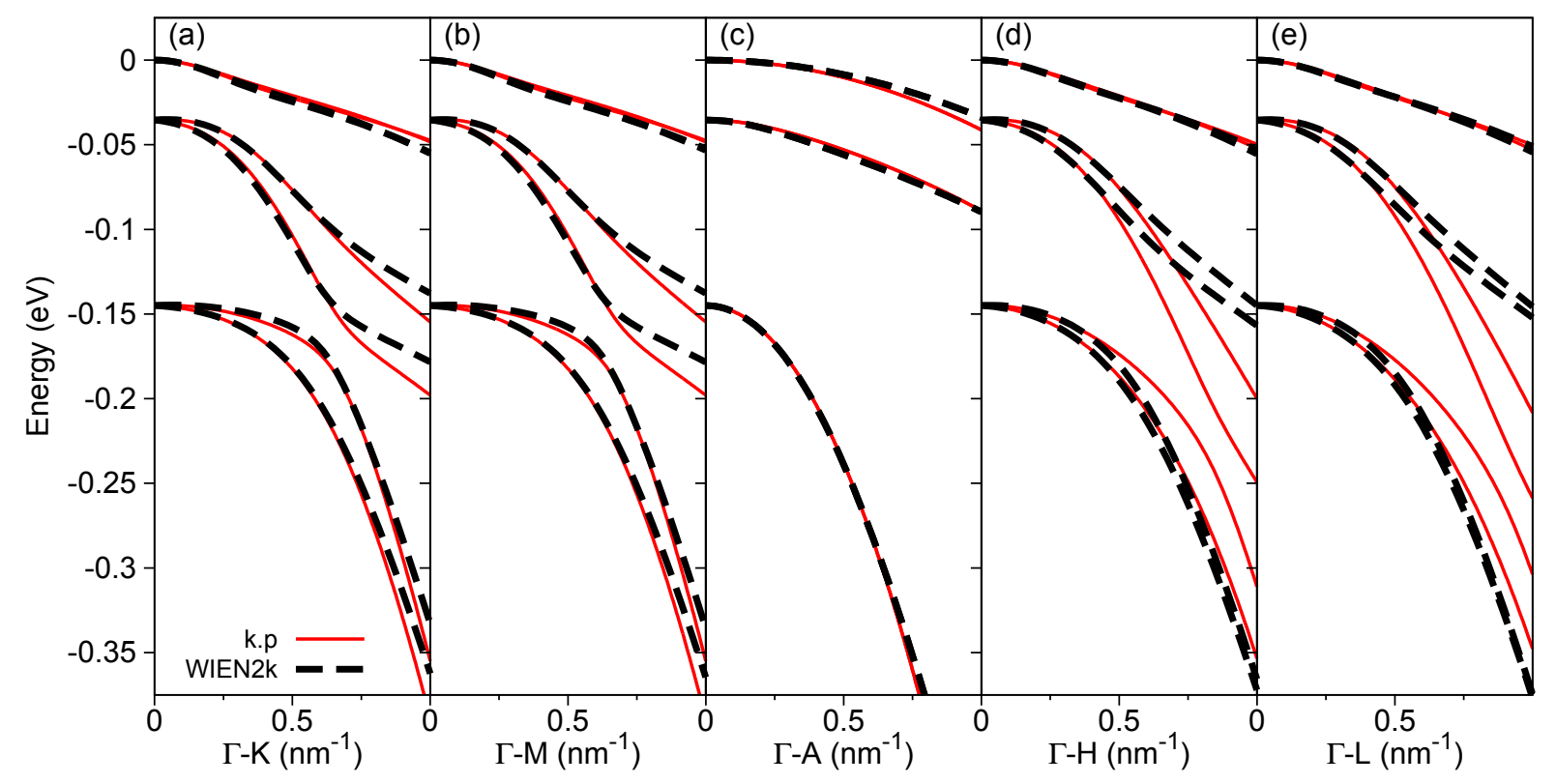

Figure 5: (Color online) Same as Fig. 2 but for InP.

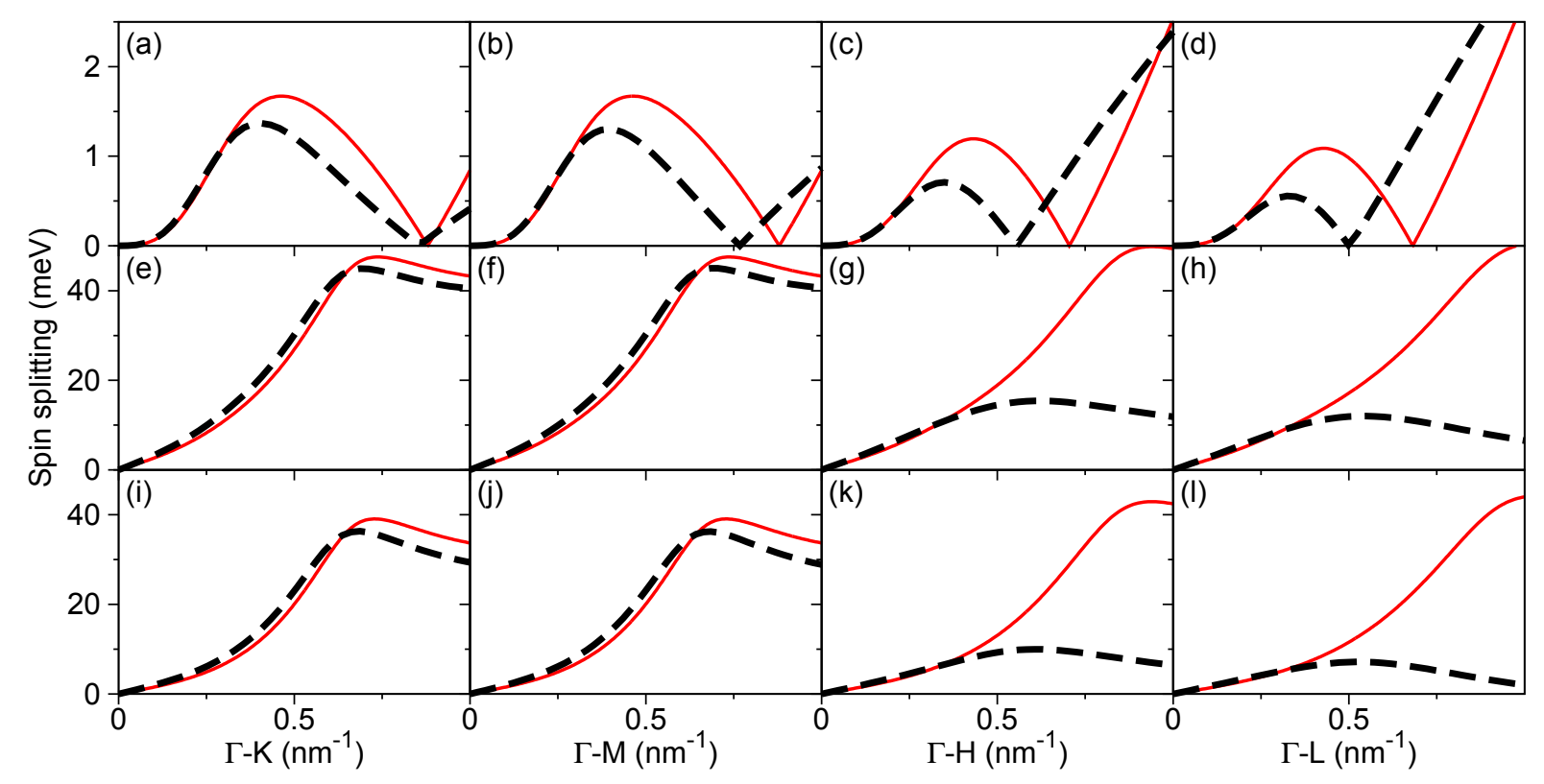

Figure 6: (Color online) Same as Fig. 3 but for InP. 

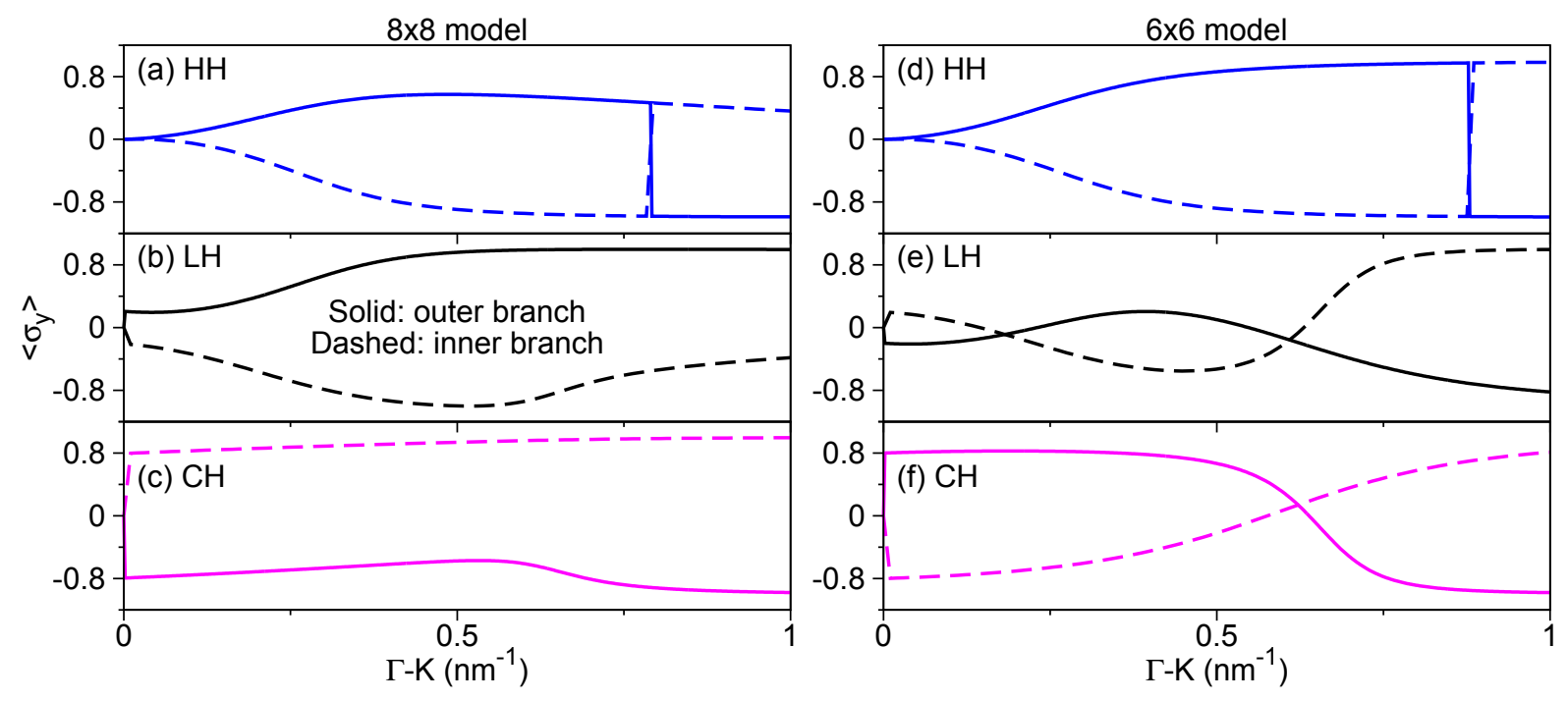

Figure 7: (Color online) Same as Fig. 4 but for InP. 


\section{Conclusions}

In this thesis we showed that the formulation of the k.p method for bulk materials and quantum confined systems can be applied to calculate reliable band structures of semiconductor systems. We studied quantum confined heterostructures of different materials with ZB crystal structure, homostructures composed of the same material with mixing of $\mathrm{ZB}$ and $\mathrm{WZ}$ phases and obtained k.p parameters of new WZ materials using a multiband state of the art Hamiltonian. Based on the calculated band structures and optical selection rules, our calculations recovered experimental features for absorption and gain coefficients and also allowed us to predict novel optical and spin-dependent properties.

Among the topics of this thesis, we investigated the operation properties of a spin laser in VCSEL geometry from a microscopic point of view(33). Specifically, we studied the effects of spin-polarized electrons, carrier density, and cavity design on the spin-dependent optical gain coefficient in the active region based on a GaAs/AlGaAs quantum well. We found that the spindependent gain coefficient is an interplay between the spin population in the band structure and the optical selection rules intrinsic to the quantum well system. Different gain values for $S^{+}$ and $S^{-}$light polarizations, i. e., the spin-filtering effect, appear as long as electrons are spin polarized. However, the largest values of gain asymmetry are not necessarily in the emission regime. Analyzing the gain values as a function of injected carrier density, we found that threshold reduction depends on the choice of the cavity design, the value of gain threshold and the spin polarization of electrons. Therefore, our microscopic calculations recover the typical steady-state properties of spin lasers found in experiments and using the rate equation approach. Regarding the possibility of high-frequency operation in spin lasers $(56,61)$, we investigated the effect of uniaxial strain-induced birefringence in the active region. We showed that small values of strain in the active region are responsible for values of birefringence coefficient, $\gamma_{p}$, above $200 \mathrm{GHz}$. Similar to the gain values, the birefringence coefficient as a function of carrier 
density strongly depends on the cavity design allowing flat line dispersions or even sign changes. Furthermore, this strain induced birefringence is ruled by the in-plane anisotropy, i. e., the injection of spin polarized carriers induces negligible changes in the $\gamma_{p}$ values. During the author proof phase of our paper(33), our predictions for large $\gamma_{p}$ values due to external strain were experimentally demonstrated in similar GaAs/AlGaAs quantum well spin laser VCSELs achieving values of $\sim 250 \mathrm{GHz}(128)$.

We also investigated in this thesis polytypic systems with ZB and WZ crystal structures using the k.p formalism. In the paper(38), we developed a polytypic k.p model based on group theory concepts that connects the irreducible representations of $\mathrm{ZB}$ and $\mathrm{WZ}$ structure at the interface $(76,95)$. Our Hamiltonian describes both ZB and WZ phases by the same matrix with the spatial dependence of parameters achieved by the EFA. In order to test the stability of our model, we applied it to InP WZ/ZB quantum wells, that show a type-II band alignment. Under quantum confinement and strain effects, our polytypic model and material parameters obtained from effective masses(95) show a typical type-II behavior, i. e., holes localized in the WZ region and electrons in the $\mathrm{ZB}$ region. We also investigated the effect of InP WZ spontaneous polarization. In the second paper about polytypism(39), we extended our polytypic model to include the linear coupling between conduction and valence bands, the term responsible for interband optical transitions. We systematically investigated the absorption spectra considering the crystal phase mixing and quantum confinement effects in a polytypic InP superlattice. We showed that for the bulk case including optical confinement (representing pure phase NWs with large diameters) the interband transition around energy gap is strongly polarized parallel (perpendicular) to the NW axis for ZB (WZ). Therefore, the DLP for pure ZB and WZ NWs show almost opposite values, in good agreement with PL measurements by Mishra et al.(79). Our calculations for the polytypic superlattices with small quantum confinement (large regions) are in good agreement with the polarized PL measurements of Gadret et al.(106), showing that even a small amount of ZB in the system induces polarization components parallel to the NW axis. In fact, for small quantum confinement the DLP is very susceptible to ZB insertion, i. e., a small amount of $10-20 \%$ of ZB shifts the DLP from -1 (perpendicular polarization) to 1 (parallel polarization). For a large quantum confinement case (small regions), the WZ features 
are retained due to the larger effective masses of holes in WZ.

Finally, we developed a realistic $8 \times 8$ k.p model to describe InAs and InP in WZ phase based on ab initio calculations from the WIEN2k code(129). Our ab initio values are in good agreement with other theoretical calculations and experimental data reported in the literature. For the k.p model, we used the single group k.p approach considering all perturbation terms in the first order, including the $k$-dependent SOC term usually neglected in the literature(118-120), and the terms $\mathbf{H}_{\mathbf{k} \mathbf{2}}+\mathbf{H}_{\mathbf{k p}}$ up to the second order. We obtain the k.p parameter sets by fitting the k.p Hamiltonian to the ab initio band structure taking into account multiple directions in the FBZ simultaneously in order to increase the accuracy of the parameter sets. The resulting k.p band structure is in good agreement with the ab initio data up to $1.0 \mathrm{~nm}^{-1}$ with deviations above this limit due to the effect of energy bands outside our model. Because of a large SOC in InAs, the labeling of valence bands (HH-CH-LH) is reversed in comparison to the usual case (HH-LH-CH) of InP. Besides the band structure, we also emphasized the spin splittings of the energy bands to obtain a good agreement with $a b$ initio. The interesting features that arise from the inversion asymmetry of the WZ structure are well described by our model, showing the importance of the $k$-dependent SOC term. Furthermore, we found that the in-plane $\left(k_{x} k_{y}\right)$ spin texture have a Rashba-like behavior, with either clockwise or counter clockwise orientation. All the spin-dependent features were systematically checked with the ab initio calculations in order to provide a realistic and robust k.p model. We also calculated the DOS for conduction and valence bands using the $8 \times 8$ model and obtained the carrier density as function of the Fermi energy. For comparison, we provided independent formulations for conduction band $(2 \times 2$ Hamiltonian $)$ and valence band $(6 \times 6$ Hamiltonian). However, we emphasize that the $8 \times 8$ model provides the best description of the ab initio band structure.

\subsection{Future possibilities}

Regarding spin lasers, our spin-dependent gain framework(33) is not limited to ZB quantum wells and, thus, can be applied to different systems. For instance, spin lasers at room temperature were recently demonstrated in GaN-based wurtzite semiconductors. $(56,151)$ Such 
wurtzite nitride compounds were recognized by the Nobel Prize in Physics of 2014 for their key role in highly-efficient LEDs.(152-154) Furthermore, the microscopic gain calculations can be coupled with the rate equations $(50,51,55,65,69-71)$, in order to provide a more realistic and systematic description of spin lasers. These gain calculations can provide a good description of the active region for different photon energies and would be considered as input parameters for the rate equations, which can address important dynamic time-dependent properties.

For semiconductor systems with non-nitride III-V WZ materials, there is still a lot of research to be done. On one hand, based on our polytypic k.p model(38, 39), we could explore band gap engineering and polytypism in other III-V compounds besides InP. For instance, there is a recent study using our polytypic k.p model to investigate GaAs ZB/WZ quantum dots(40). On the other hand, in order to provide a systematic investigation of polytypism, it is crucial to have k.p parameters for these new possibilities of WZ materials. Our recent paper(127) attempts to remove such deficiencies by providing parameter sets for InAs and InP WZ materials to be used in multiband k.p Hamiltonians including $k$-dependent SOC effects. As fas as we are concerned, this is the first paper in the literature to provide a robust set of parameters for InP and InAs WZ. Our k.p framework, combined with the EFA, provides a theoretical reliable approach to investigate not only polytypism but also other interesting physical phenomena such as charge and spin transport, optics and proximity effects. 


\section{References}

1 ASHCROFT, N. W.; MERMIN, N. D. Solid state physics. Harcourt: Brooks Cole, 1976.

2 HODDESON, L. et al. Out of the crystal maze: chapters from the history of solid-state physics. Oxford: Oxford University Press, 1992.

3 MARTIN, R. M. Electronic structure: basic theory and practical methods. Cambridge: Cambridge University Press, 2004.

4 YU, P. Y.; CARDONA, M. Fundamentals of semiconductors: physics and materials properties. Berlin: Springer, 2001.

5 KUNEŠ, J. et al. Wien2wannier: from linearized augmented plane waves to maximally localized Wannier functions. Computer Physics Communications, v. 181, n. 11, p. 1888, 2010.

6 VOON, L. C. L. Y.; WILLATZEN, M. The k.p method: electronic properties of semiconductors. Berlin: Springer, 2009.

7 PRYOR, C. E.; PISTOL, M.-E. Atomistic k.p theory. Journal of Applied Physics, v. 118, n. 22, p. 225702, 2015.

8 LÖWDIN, P. A note on the quantum-mechanical perturbation theory. The Journal of Chemical Physics, v. 19, n. 11, p. 1396, 1951.

9 LUTTINGER, J. M.; KOHN, W. Motion of electrons and holes in perturbed periodic fields. Physical Review, v. 97, n. 4, p. 869, 1955.

10 DRESSELHAUS, G. Spin-orbit coupling effects in zinc blend structures. Physical Review, v. 100 , n. 2 , p. $580,1955$.

11 KANE, E. O. Physics of III-V compounds. New York: Academic Press, 1966. (Semiconductors and Semimetals, v. 1).

12 DRESSELHAUS, M. S.; DRESSELHAUS, G.; JORIO, A. Group theory: application to the physics of condensed matter. Berlin: Springer, 2008. 
13 FAZZIO, A.; WATARI, K. Introdução à teoria de grupos: aplicada em moléculas e sólidos. Santa Maria: Editora da Universidade Federal de Santa Maria, 2009.

14 BASTARD, G. Superlattice band structure in the envelope-function approximation. Physical Review B, v. 24, n. 10, p. 5692, 1981.

15 BURT, M. G. An exact formulation of the envelope function method for the determination of electronic states in semiconductor microstructures. Semiconductor Science and Technology, v. 2, n. 7, p. 460, 1987.

16 BURT, M. G. A new effective-mass equation for microstructures. Semiconductor Science and Technology, v. 3, n. 12, p. 1224, 1988.

17 BARAFF, G. A.; GERSHONI, D. Eigenfunction-expansion method for solving the quantum-wire problem: Formulation. Physical Review B, v. 43, n. 5, p. 4011, 1991.

18 BURT, M. G. The justification for applying the effective-mass approximation to microstructures. Journal of Physics: condensed matter, v. 4, n. 32, p. 6651, 1992.

19 FOREMAN, B. A. Envelope-function formalism for electrons in abrupt heterostructures with material-dependent basis functions. Physical Review B, v. 54, n. 3, p. 1909, 1996.

20 ŽUTIĆ, I.; FABIAN, J.; DAS SARMA, S. Spintronics: fundamentals and applications. Reviews of Modern Physics, v. 76, n. 2, p. 323, 2004.

21 FABIAN, J. et al. Semiconductor spintronics. Acta Physica Slovaca, v. 57, n. 4, p. 565, 2007.

22 DE ANDRADA E SILVA, E. A.; ROCCA, G. C. L.; BASSANI, F. Spin-orbit splitting of electronic states in semiconductor asymmetric quantum wells. Physical Review B, v. 55, n. 24, p. 16293, 1997.

23 BERNARDES, E. et al. Spin-orbit interaction in symmetric wells with two subbands. Physical Review Letters, v. 99, n. 7, p. 076603, 2007.

24 CALSAVERINI, R. S. et al. Intersubband-induced spin-orbit interaction in quantum wells. Physical Review B, v. 78, n. 15, p. 155313, 2008.

25 DOS SANTOS, L. F. et al. Quantum oscillations of spin polarization in a GaAs/AlGaAs double quantum well. Physical Review B, v. 86, n. 12, p. 125415, 2012.

26 PUSEP, Y. A. et al. Circularly polarized photoluminescence as a probe of density of states in GaAs/AlGaAs quantum hall bilayers. Physical Review Letters, v. 109, n. 4, p. 046802, 2012. 
27 FU, J.; EGUES, J. C. Spin-orbit interaction in GaAs wells: from one to two subbands. Physical Review B, v. 91, n. 7, p. 075408, 2015.

28 NASTOS, F. et al. Full band structure LDA and k.p calculations of optical spin-injection. Physical Review B, v. 76, n. 20, p. 205113, 2007.

29 RIOUX, J.; SIPE, J. E. Optical injection processes in semiconductors. Physica E, v. 45, n. 0 , p. 1, 2012.

30 BOTTEGONI, F. et al. Enhanced orbital mixing in the valence band of strained germanium. Physical Review B, v. 85, n. 24, p. 245312, 2012.

31 PEZZOLI, F. et al. Optical spin injection and spin lifetime in Ge heterostructures. Physical Review Letters, v. 108, n. 15, p. 156603, 2012.

32 HOLUB, M.; JONKER, B. T. Threshold current reduction in spin-polarized lasers: role of strain and valence-band mixing. Physical Review B, v. 83, n. 12, p. 125309, 2011.

33 FARIA JUNIOR, P. E. et al. Toward high-frequency operation of spin lasers. Physical Review B, v. 92, n. 7, p. 075311, 2015.

34 NOVIK, E. G. et al. Band structure of semimagnetic $\mathrm{Hg}_{1-y} \mathrm{Mn}_{y}$ Te quantum wells. Physical Review B, v. 72, n. 3, p. 035321, 2005.

35 BERNEVIG, B. A.; HUGHES, T. L.; ZHANG, S.-C. Quantum spin hall effect and topological phase transition in HgTe quantum wells. Science, v. 314, n. 5806, p. 1757, 2006.

36 MIAO, M. S. et al. Polarization-driven topological insulator transition in a GaN/InN/GaN quantum well. Physical Review Letters, v. 109, n. 18, p. 186803, 2012.

37 ERLINGSSON, S. I.; EGUES, J. C. All-electron topological insulator in InAs double wells. Physical Review B, v. 91, n. 3, p. 035312, 2015.

38 FARIA JUNIOR, P. E.; SIPAHI, G. M. Band structure calculations of InP wurtzite/zincblende quantum wells. Journal of Applied Physics, v. 112, n. 10, p. 103716, 2012.

39 FARIA JUNIOR, P. E.; CAMPOS, T.; SIPAHI, G. M. Interband polarized absorption in InP polytypic superlattices. Journal of Applied Physics, v. 116, n. 19, p. 193501, 2014.

40 CLIMENTE, J. I. et al. Electrons, holes, and excitons in GaAs polytype quantum dots. Journal of Applied Physics, v. 119, n. 12, p. 125705, 2016.

41 LI, P.; APPELBAUM, I. Electrons and holes in phosphorene. Physical Review B, v. 90, n. 11, p. 115439, 2014. 
42 RODIN, A. S.; CARVALHO, A.; CASTRO NETO, A. H. Strain-induced gap modification in black phosphorus. Physical Review Letters, v. 112, n. 17, p. 176801, 2014.

43 KORMÁNYOS, A. et al. k.p theory for two-dimensional transition metal dichalcogenide semiconductors. 2D Materials, v. 2, n. 2, p. 022001, 2015.

44 PARKER, M. A. Physics of optoelectronics. New York: CRC Press, 2005.

45 LASER facts. Available at: 〈http://www.nobelprize.org/educational/physics/laser/facts/ history.html $\rangle$. Accessible at: 03/25/2016.

46 ALFEROV, Z. I. Nobel lecture: the double heterostructure concept and its applications in physics, electronics, and technology. Reviews of Modern Physics, v. 73, n. 3, p. 767, 2001.

47 KLOTZKIN, D. J. Introduction to semiconductor lasers for optical communications: an applied approach. New York: Springer, 2014.

48 MEIER, F.; ZAKHARCHENYA, B. P. Optical orientation. New York: North Holland, 1984.

49 TSYMBAL, E. Y.; ŽUTIĆ, I. Handbook of spin transport and magnetism. New York: Chapman and Hall/CRC, 2011.

50 RUDOLPH, J. et al. Laser threshold reduction in a spintronic device. Applied Physics Letters, v. 82, n. 25, p. 4516, 2003.

51 RUDOLPH, J. et al. Room-temperature threshold reduction in vertical-cavity surfaceemitting lasers by injection of spin-polarized electrons. Applied Physics Letters, v. 87, n. 24, p. 241117, 2005.

52 HOLUB, M. et al. Electrical spin injection and threshold reduction in a semiconductor laser. Physical Review Letters, v. 98, n. 14, p. 146603, 2007.

53 SAHA, D.; BASU, D.; BHATTACHARYA, P. High-frequency dynamics of spin-polarized carriers and photons in a laser. Physical Review B, v. 82, n. 20, p. 205309, 2010.

54 IBA, S. et al. Room temperature circularly polarized lasing in an optically spin injected vertical-cavity surface-emitting laser with (110) GaAs quantum wells. Applied Physics Letters, v. 98 , n. 8, p. 081113, 2011.

55 LEE, J. et al. Mapping between quantum dot and quantum well lasers: from conventional to spin lasers. Physical Review B, v. 85, n. 4, p. 045314, 2012. 
56 ŽUTIĆ, I.; FARIA JUNIOR, P. E. Semiconductor lasers: taken for a spin. Nature Nanotechnology, v. 9, n. 10, p. 750, 2014.

57 MICHALZIK, R. VCSELs: fundamentals, technology and applications of vertical-cavity surface-emitting lasers. Berlin: Springer, 2013.

58 BASU, D. et al. Electrically injected InAs/GaAs quantum dot spin laser operating at 200 K. Applied Physics Letters, v. 92, n. 9, p. 091119, 2008.

59 BASU, D.; SAHA, D.; BHATTACHARYA, P. Optical polarization modulation and gain anisotropy in an electrically injected spin laser. Physical Review Letters, v. 102, n. 9, p. 093904 , 2009.

60 LI, M. Y. et al. Birefringence controlled room-temperature picosecond spin dynamics close to the threshold of vertical-cavity surface-emitting laser devices. Applied Physics Letters, v. 97, n. 19, p. 191114, 2010.

61 GERHARDT, N. C. et al. Ultrafast spin-induced polarization oscillations with tunable lifetime in vertical-cavity surface-emitting lasers. Applied Physics Letters, v. 99, n. 15, p. 151107, 2011.

62 FROUGIER, J. et al. Control of light polarization using optically spin-injected vertical external cavity surface emitting lasers. Applied Physics Letters, v. 103, n. 25, p. 252402, 2013.

63 FROUGIER, J. et al. Accurate measurement of the residual birefringence in VECSEL: towards understanding of the polarization behavior under spin-polarized pumping. Optics Express, v. 23, n. 8, p. 9573, 2015.

64 ALHARTHI, S. S. et al. $1300 \mathrm{~nm}$ optically pumped quantum dot spin vertical externalcavity surface-emitting laser. Applied Physics Letters, v. 107, n. 15, p. 151109, 2015.

65 LEE, J. et al. Spin modulation in semiconductor lasers. Applied Physics Letters, v. 97, n. 4 , p. $041116,2010$.

66 CHUANG, S. L. Physics of optoelectronic devices. New York: John Wiley, 1995.

67 HÖPFNER, H. et al. Controlled switching of ultrafast circular polarization oscillations in spin-polarized vertical-cavity surface-emitting lasers. Applied Physics Letters, v. 104, n. 2, p. 022409, 2014.

68 LINDEMANN, M. et al. Frequency tuning of polarization oscillations: toward high-speed spin-lasers. Applied Physics Letters, v. 108, n. 4, p. 042404, 2016. 
69 OESTREICH, M. et al. Design considerations for semiconductor spin lasers. Superlattices and Microstructures, v. 37, n. 5, p. 306, 2005.

70 GØTHGEN, C. et al. Analytical model of spin-polarized semiconductor lasers. Applied Physics Letters, v. 93, n. 4, p. 042513, 2008.

71 LEE, J. et al. Spin-lasers: from threshold reduction to large-signal analysis. Applied Physics Letters, v. 105, n. 4, p. 042411, 2014.

72 MIGUEL, M. S.; FENG, Q.; MOLONEY, J. V. Light-polarization dynamics in surfaceemitting semiconductor lasers. Physical Review A, v. 52, n. 2, p. 1728, 1995.

73 MULET, J.; BALLE, S. Spatio-temporal modeling of the optical properties of VCSELs in the presence of polarization effects. IEEE Journal of Quantum Electronics, v. 38, n. 3, p. 291, 2002.

74 ADACHI, S. Physical properties of III-V semiconductor compounds: InP, InAs, GaAs, GaP, InGaAs, and InGaAsP. Chichester: John Wiley, 1992.

75 YEH, C.-Y. et al. Zinc-blende/wurtzite polytypism in semiconductors. Physical Review B, v. 46, n. 16, p. 10086, 1992.

76 MURAYAMA, M.; NAKAYAMA, T. Chemical trend of band offsets at wurtzite/zincblende heterocrystalline semiconductor interfaces. Physical Review B, v. 49, n. 7, p. 4710, 1994.

77 MCMAHON, M. I.; NELMES, R. J. Observation of a wurtzite form of gallium arsenide. Physical Review Letters, v. 95, n. 21, p. 215505, 2005.

78 JOHANSSON, J. et al. Structural properties of $\langle 111\rangle$ B-oriented III-V nanowires. Nature Materials, v. 5, n. 7, p. 574, 2006.

79 MISHRA, A. et al. Polarization and temperature dependence of photoluminescence from zincblende and wurtzite InP nanowires. Applied Physics Letters, v. 91, n. 26, p. 263104, 2007.

80 BAO, J. et al. Nanowire-induced wurtzite InAs thin film on zinc-blende InAs substrate. Advanced Materials, v. 21, n. 36, p. 3654, 2009.

81 CAROFF, P. et al. Controlled polytypic and twin-plane superlattices in III-V nanowires. Nature Nanotechnology, v. 4, n. 1, p. 50, 2009.

82 DUBROVSKII, V. G.; CIRLINA, G. E.; USTINOV, V. M. Semiconductor nanowhiskers: synthesis, properties, and applications. Semiconductors, v. 43, n. 12, p. 1585, 2009. 
83 DICK, K. A. et al. Crystal phase engineering in single InAs nanowires. Nano Letters, v. 10, n. 9, p. 3494, 2010.

84 WAGNER, R. S.; ELLIS, W. C. Vapor-liquid-solid mechanism of single crystal growth. Applied Physics Letters, v. 4, n. 5, p. 89, 1964.

85 VU, T. T. T. et al. High optical quality single crystal phase wurtzite and zincblende InP nanowires. Nanotechnology, v. 24, n. 11, p. 115705, 2013.

86 PAN, D. et al. Controlled synthesis of phase-pure InAs nanowires on $\mathrm{Si}(111)$ by diminishing the diameter to $10 \mathrm{~nm}$. Nano Letters, v. 14, n. 3, p. 1214, 2014.

87 BOLINSSON, J. et al. Wurtzite-zincblende superlattices in InAs nanowires using a supply interruption method. Nanotechnology, v. 22, n. 26, p. 265606, 2011.

88 LEHMANN, S. et al. A general approach for sharp crystal phase switching in InAs, GaAs, InP, and GaP nanowires using only group V flow. Nano Letters, v. 13, n. 9, p. 4099, 2013.

89 JACOBSSON, D. et al. Phase transformation in radially merged wurtzite GaAs nanowires. Crystal Growth \& Design, v. 15, n. 10, p. 4795, 2015.

90 JOHANSSON, J.; ZANOLLI, Z.; DICK, K. A. Polytype attainability in III-V semiconductor nanowires. Crystal Growth \& Design, v. 16, n. 1, p. 371, 2016.

91 THELANDER, C. et al. Effects of crystal phase mixing on the electrical properties of InAs nanowires. Nano Letters, v. 11, n. 6, p. 2424, 2011.

92 WANG, Z. et al. Polytypic InP nanolaser monolithically integrated on (001) silicon. Nano Letters, v. 13, n. 11, p. 5063, 2013.

93 HOANG, T. B. et al. Engineering parallel and perpendicular polarized photoluminescence from a single semiconductor nanowire by crystal phase control. Nano Letters, v. 10, n. 8, p. 2927, 2010.

94 ZANOLLI, Z. et al. Model GW band structure of InAs and GaAs in the wurtzite phase. Physical Review B, v. 75, n. 24, p. 245121, 2007.

95 DE, A.; PRYOR, C. E. Predicted band structures of III-V semiconductors in the wurtzite phase. Physical Review B, v. 81, n. 15, p. 155210, 2010.

96 DACAL, L. C.; CANTARERO, A. Ab initio electronic band structure calculation of InP in the wurtzite phase. Solid State Communications, v. 151, n. 10, p. 781, 2011. 
97 BELABBES, A. et al. Electronic bands of III-V semiconductor polytypes and their alignment. Physical Review B, v. 86, n. 7, p. 075208, 2012.

98 HAJLAOUI, C. et al. First-principles density functional theory study of strained wurtzite InP and InAs. Journal of Physics D: applied physics, v. 46, n. 50, p. 505106, 2013.

99 DACAL, L. C. O.; CANTARERO, A. Ab initio calculations of indium arsenide in the wurtzite phase: structural, electronic and optical properties. Materials Research Express, v. 1, n. 1, p. 015702, 2014.

100 VURGAFTMAN, I.; MEYER, J. R.; RAM-MOHAN, L. R. Band parameters for III-V compound semiconductors and their alloys. Journal of Applied Physics, v. 89, n. 11, p. 5815, 2001.

101 OREG, Y.; REFAEL, G.; VON OPPEN, F. Helical liquids and Majorana bound states in quantum wires. Physical Review Letters, v. 105, n. 17, p. 177002, 2010.

102 MOURIK, V. et al. Signatures of Majorana fermions in hybrid superconductorsemiconductor nanowire devices. Science, v. 336, n. 6084, p. 1003, 2012.

103 DENG, M. T. et al. Anomalous zero-bias conductance peak in a Nb-InSb nanowire- Nb hybrid device. Nano Letters, v. 12, n. 12, p. 6414, 2012.

104 DAS, A. et al. Zero-bias peaks and splitting in an Al-InAs nanowire topological superconductor as a signature of Majorana fermions. Nature Physics, v. 8, n. 12, p. 887, 2012.

105 ALBRECHT, S. M. et al. Exponential protection of zero modes in Majorana islands. Nature, v. 531, n. 7593, p. 206, 2016.

106 GADRET, E. G. et al. Valence-band splitting energies in wurtzite InP nanowires: photoluminescence spectroscopy and ab initio calculations. Physical Review B, v. 82, n. 12, p. $125327,2010$.

107 ZILLI, A. et al. Temperature dependence of interband transitions in wurtzite InP nanowires. ACS Nano, v. 9, n. 4, p. 4277, 2015.

108 DE LUCA, M. et al. Polarized light absorption in wurtzite InP nanowire ensembles. Nano Letters, v. 15, n. 2, p. 998, 2015.

109 DE LUCA, M. et al. Magneto-optical properties of wurtzite-phase InP nanowires. Nano Letters, v. 14, n. 8, p. 4250, 2014.

110 WINKLER, R. Spin-orbit coupling effects in two-dimensional electron and hole systems. Berlin: Springer, 2003. 
111 TOLOZA SANDOVAL, M. A. et al. Mesoscopic spin-orbit effect in the semiconductor nanostructure electron $g$ factor. Physical Review B, v. 86, n. 19, p. 195302, 2012.

112 RIDOLFI, E.; DE ANDRADA E SILVA, E. A.; LA ROCCA, G. C. Effective $g$-factor tensor for carriers in IV-VI semiconductor quantum wells. Physical Review B, v. 91, n. 8, p. $085313,2015$.

113 RASHBA, E. I.; SHEKA, V. I. Symmetry of energy bands in crystals of wurtzite type: II. Symmetry of bands including spin-orbit interaction. Fizika Tverdogo Tela, v. 1, n. 2, p. 162, 1959.

114 BYCHKOV, Y. A.; RASHBA, E. I. Properties of a 2D electron gas with lifted spectral degeneracy. JETP Letters, v. 39, n. 2, p. 78, 1984.

115 BIHLMAYER, G.; RADER, O.; WINKLER, R. Focus on the rashba effect. New Journal of Physics, v. 17, n. 5, p. 050202, 2015.

116 DATTA, S.; DAS, B. Electronic analog of the electro-optic modulator. Applied Physics Letters, v. 56, n. 7, p. 665, 1990.

117 CARDONA, M.; CHRISTENSEN, N. E.; FASOL, G. Relativistic band structure and spin-orbit splitting of zinc-blende-type semiconductors. Physical Review B, v. 38, n. 3, p. 1806, 1988.

118 CHUANG, S. L.; CHANG, C. S. k·p method for strained wurtzite semiconductors. Physical Review B, v. 54, n. 4, p. 2491, 1996.

119 FU, J. Y.; WU, M. W. Spin-orbit coupling in bulk ZnO and GaN. Journal of Applied Physics, v. 104, n. 9, p. 093712, 2008.

120 CHEIWCHANCHAMNANGIJ, T.; LAMBRECHT, W. R. L. Band structure parameters of wurtzite and zinc-blende GaAs under strain in the GW approximation. Physical Review B, v. 84, n. 3, p. $035203,2011$.

121 BERESFORD, R. Full-zone k.p method of band structure calculation for wurtzite semiconductors. Journal of Applied Physics, v. 95, n. 11, p. 6216, 2004.

122 RINKE, P. et al. Consistent set of band parameters for the group-III nitrides AlN, GaN, and InN. Physical Review B, v. 77, n. 7, p. 075202, 2008.

123 MARNETTO, A.; PENNA, M.; GOANO, M. An accurate dual-expansion-point fullbrillouin-zone k.p model for wurtzite semiconductors. Journal of Applied Physics, v. 108, n. 3, p. 033701, 2010. 
124 PUNYA, A.; LAMBRECHT, W. R. L. Valence band effective-mass hamiltonians for the group-III nitrides from quasiparticle self-consistent GW band structures. Physical Review B, v. 85, n. 19 , p. 195147, 2012.

125 BASTOS, C. M. O. Determinação de parâmetros para Hamiltonianos k.p a partir de estruturas de bandas pré-existentes. 2015.97p. Dissertação (Mestrado em Ciências) — Instituto de Física de São Carlos, Universidade de São Paulo, São Carlos, 2015.

126 BASTOS, C. M. O. et al. Stability and accuracy control of k.p parameters. Semiconductor Science and Technology, 2016. In press.

127 FARIA JUNIOR, P. E. et al. Realistic multiband $k \cdot p$ approach from $a b$ initio and spinorbit coupling effects of InAs and InP in wurtzite phase. Physical Review B, v. 93, n. 23, p. 235204, 2016.

128 PUSCH, T. et al. Vertical-cavity surface-emitting lasers with birefringence splitting above 250 GHz. Electronics Letters, v. 51, n. 20, p. 1600, 2015.

129 BLAHA, P. et al. WIEN2k: an augmented olane wave + local orbitals program for calculating crystal properties. Vienna: University of Technology, 2013.

130 ENDERLEIN, R.; HORING, N. J. M. Fundamentals of semiconductor physics and devices. Singapore: World Scientific, 1997.

131 COHEN-TANNOUDJI, C.; DIU, B.; LALÖ̈, F. Quantum mechanics. New York: John Wiley, 1977, v. 2.

132 CASELLA, R. C. Symmetry of wurtzite. Physical Review, v. 114, n. 6, p. 1514, 1959.

133 ELDER, W. J.; WARD, R. M.; ZHANG, J. Double-group formulation of k.p theory for cubic crystals. Physical Review B, v. 83, n. 16, p. 165210, 2011.

134 FARIA JUNIOR, P. E. Nanowhiskers politípicos - uma abordagem teórica baseada em teoria de grupos e no método k.p. 2012. 138p. Dissertação (Mestrado em Ciências) — Instituto de Física de São Carlos, Universidade de São Paulo, São Carlos, 2012.

135 CANDIDO, D. R. Relaxação de spin via D'yakonov-Perel' em poços quânticos com acoplamento spin-órbita intersub-banda. 2013. 227p. Dissertação (Mestrado em Ciências) Instituto de Física de São Carlos, Universidade de São Paulo, São Carlos, 2013.

136 DRESSELHAUS, G.; KIP, A. F.; KITTEL, C. Cyclotron resonance of electrons and holes in silicon and germanium crystals. Physical Review, v. 98, n. 2, p. 368, 1955. 
137 NEWVILLE, M. et al. LMFIT: non-linear least-square minimization and curve-fitting for Python $\uparrow$. Zenodo, 2014. Available at: $\langle$ http://dx.doi.org/10.5281/zenodo.11813 $\rangle$. Accessible at: 05/19/2016.

138 SIPAHI, G. M. et al. Theory of luminescence spectra from $\delta$-doping structures: application to GaAs. Physical Review B, v. 57, n. 15, p. 9168, 1998.

139 ANDREANI, L. C.; PASQUARELLO, A.; BASSANI, F. Hole subbands in strained GaAs- $\mathrm{Ga}_{1-\mathrm{x}} \mathrm{Al}_{\mathrm{x}}$ As quantum wells: exact solution of the effective-mass equation. Physical Review $B$, v. 36, n. 11, p. 5887, 1987.

140 CHUANG, S. L.; CHANG, C. S. A band-structure model of strained quantum-well wurtzite semiconductors. Semiconductor Science and Technology, v. 12, n. 3, p. 252, 1997.

141 ISHIGAKI, T. et al. Analysis of subband structures and optical properties of periodic strained quantum wires by a finite element method. Journal of Applied Physics, v. 91, n. 9, p. 5815, 2002.

142 CAMPOS, T. Nanowires de InP: cálculo do espectro de absorção via método k.p. 2013. 119p. Dissertação (Mestrado em Ciências) — Instituto de Física de São Carlos, Universidade de São Paulo, São Carlos, 2013.

143 KITTEL, C. Introduction to solid state physics. New York: John Wiley, 1996.

144 CHOW, W. W.; KOCH, S. W. Semiconductor-laser fundamentals: physics of the gain materials. New York: Springer, 1999.

145 HAUG, H.; KOCH, S. W. Quantum theory of optical and electronic properties of semiconductors. Singapore: World Scientific, 2004.

146 COLOCCI, M. et al. Temperature dependence of exciton lifetimes in GaAs/AlGaAs quantum well structures. Europhysics Letters, v. 12, n. 5, p. 417, 1990.

147 DE DIOS-LEYVA, M.; OLIVEIRA, L. E. Carrier densities and electron-hole recombination lifetimes in GaAs-(Ga,Al)As quantum-well photoluminescence. Journal of Applied Physics, v. 75, n. 1, p. 660, 1994.

148 DYAKONOV, M. I.; PEREL, V. I. Spin relaxation of conduction electrons in noncentrosymmetric semiconductors. Soviet Physics Solid State, v. 13, n. 12, p. 3023, 1972.

149 HACHIYA, M. A. O. Spin relaxation in semiconductor nanostructures. 2013. 114p. Tese (Doutorado) - Instituto de Física de São Carlos, Universidade de São Paulo, São Carlos, 2013. 
150 KAINZ, J.; RÖSSLER, U.; WINKLER, R. Temperature dependence of Dyakonov-Perel spin relaxation in zinc-blende semiconductor quantum structures. Physical Review B, v. 70, n. 19, p. 195322, 2004.

151 CHEN, J.-Y. et al. Self-polarized spin-nanolasers. Nature Nanotechnology, v. 9, n. 10, p. 845, 2014.

152 NAKAMURA, S. Nobel lecture: background story of the invention of efficient blue InGaN light emitting diodes. Reviews of Modern Physics, v. 87, n. 4, p. 1139, 2015.

153 AMANO, H. Nobel lecture: growth of GaN on sapphire via low-temperature deposited buffer layer and realization of $p$-type $\mathrm{GaN}$ by $\mathrm{Mg}$ doping followed by low-energy electron beam irradiation. Reviews of Modern Physics, v. 87, n. 4, p. 1133, 2015.

154 AKASAKI, I. Nobel lecture: fascinated journeys into blue light. Reviews of Modern Physics, v. 87, n. 4, p. 1119, 2015. 